\title{
Solution-Phase Synthesis of Nanoparticles and Growth Study
}

\author{
By \\ Soshan Cheong
}

\begin{abstract}
A thesis
submitted to Victoria University of Wellington in fulfilment of the requirements for the degree of

Doctor of Philosophy in Chemistry
\end{abstract}

Victoria University of Wellington

2010 


\section{Acknowlegements}

The accomplishments of this thesis involve the contributions of many people in one way or the other, whom I would very much like to acknowledge:

My PhD supervisor Dr. Richard Tilley, for his guidance and support in every aspect of the research, and also for the various research opportunities he has given me.

My secondary supervisor Dr. Shaun Hendy, for his kind encouragement, and the access to the main financial support for this research work.

Dr. Michael Toney (Stanford Synchrotron Radiation Lightsource) and

Dr. Bridget Ingham (Industrial Research Ltd.), for their help in conducting the synchrotron experiments, and their discussions and contributions leading to the publication of the study.

Dr. Claire Meyer (Neel Institute, Grenoble), for her kind help and assistance in conducting magnetic measurements.

Prof. Joe Trodahl, for his stimulating scientific discussions and ideas, and good humour.

Alan Rennie and Manu Pouajen-Blakiston, for their great help in solving and fixing various problems encountered with the Fischer-Porter bottle setups, and for fabricating the XRD cell.

David Flynn, for looking after the electron microscopes and help in the SEM.

Past and present members of RDT group. In particular, Jintian Ren, for sharing his experience in Fischer-Porter bottle synthesis; Dr. Wendy Popplewell, for her help in the synthesis of iron-based nanoparticles and in preparing the iron precursor; John Watt and Sujay Prabakar, for stimulating and interesting discussions, and friendships of all.

My family especially my parents and my sister for their unconditional support and encouragement.

I would also like to thank Dr. Tilley, Dr. Popplewell, Dr. Kirsten Edgar and Aivee Lim for proofreading part of this thesis.

I would like to acknowledge the following financial sources:

The RSNZ Marsden Fund IRL0602, led by Dr. Shaun Hendy.

The Science Faculty Research Grant of Victoria University. 


\section{Abstract}

This thesis is concerned with solution-phase synthesis of nanoparticles and growth of nanoparticles in solution. A facile synthesis route was developed to produce nanoparticles of iron, iron carbide and ruthenium. In general, the synthesis involved the reaction/decomposition of a metal precursor in solution, in the presence of a stabilising agent, in a closed reaction vessel, under a hydrogen atmosphere. The crystallinity, crystal structure, morphology and chemical composition of the nanoparticles obtained were studied primarily by transmission electron microscopy (TEM), selected area electron diffraction (SAED), powder X-ray diffraction (XRD), and energy dispersive X-ray spectroscopy (EDS). Scanning quantum interference device magnetometry (SQUID) was used to characterise the magnetic properties of iron and iron carbide nanoparticles. In situ synchrotron-based XRD was employed to investigate the growth of platinum nanoparticles of different morphologies.

The synthesis of iron and iron carbide nanoparticles was investigated at temperatures $80-160{ }^{\circ} \mathrm{C}$. Syntheses at $130^{\circ} \mathrm{C}$ and above produced mainly single-crystal $\alpha$-Fe nanoparticles, whereas those at lower temperatures yielded products consisting of $\alpha$-Fe and $\mathrm{Fe}_{3} \mathrm{C}$ nanoparticles. Nanoparticles of larger than $10 \mathrm{~nm}$ oxidised on the surface leading to core/shell structures, and those of smaller size oxidised completely upon exposure to air. Core/shell nanoparticles of larger than $15 \mathrm{~nm}$ were observed to be stable under ambient conditions for at least a year, whereas those smaller in size underwent further oxidation forming core/void/shell structures. The magnetic properties of selected samples were characterised. The core/shell nanoparticles were shown to exhibit ferromagnetic behaviours, and saturation magnetisations were obtained at the range of 100-130 emu g-1.

Nanoparticle size and size distribution, and morphology were found to be a result of combined effect of precursor concentration and the relative stabiliser concentration. In general, high-precursor concentration resulted in less controlled reaction and produced large nanoparticle size and size distribution. Under the high-concentration condition, the use of stabilisers in reduced amount then led to a diverse range of morphologies, which include dimer, porous and branched structures. 
As for the synthesis of ruthenium nanoparticles, reactions of different precursors were investigated at temperatures ranging from room temperature to $140{ }^{\circ} \mathrm{C}$. Highly crystalline ruthenium nanoparticles of different sizes and morphologies were obtained through different experimental conditions. The increase in nanoparticle size was found to be a result of increasing reaction temperature and/or decreasing stabiliser to ruthenium ratio. This trend was observed to be independent of the type of stabilisers and precursors used. The use of stabilisers with different binding characteristics has facilitated the formation of non-spherical nanoparticles; these include rod-like structures with high aspect ratios (of up to 12), hexagonal and truncated triangular plate-like structures, and tripods.

The growth of faceted and branched structures of platinum nanoparticles was investigated by employing in situ XRD techniques. TEM was used to examine the intermediate structures. The two different morphologies were previously shown to be governed by precursor concentration. It was found that the growth in the low-concentration reaction was characteristic of a thermodynamically controlled regime, whereas that in the high-concentration reaction occurred at much greater rates under a kinetically controlled regime. Based on the observations obtained, different growth mechanisms were proposed and discussed. The former involved an oriented attachment mechanism, while the latter, a novel mechanism involving selective growth and etching processes.

The results are followed by an overall discussion comparing and contrasting the various syntheses involved, and relating the results of syntheses to those of the growth studies. 


\section{Contents}

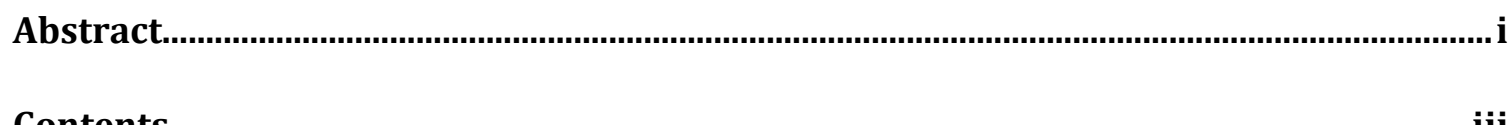

List of Figures

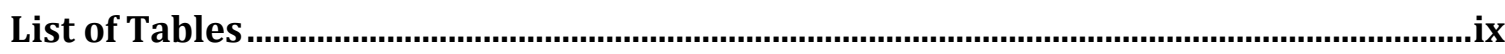

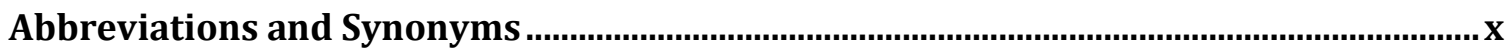

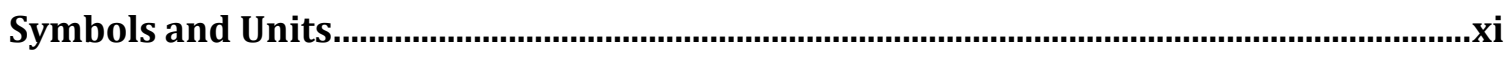

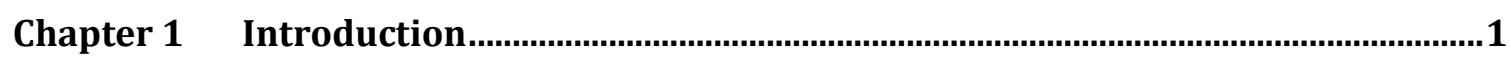

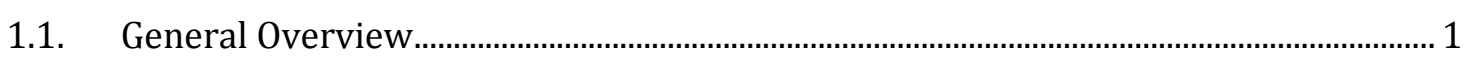

1.2. Solution-Phase Synthesis of Nanoparticles ....................................................................... 2

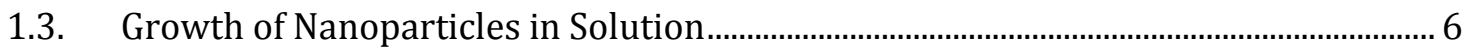

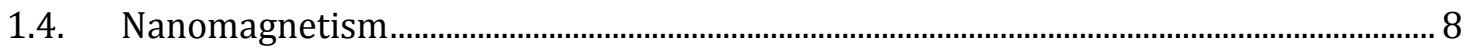

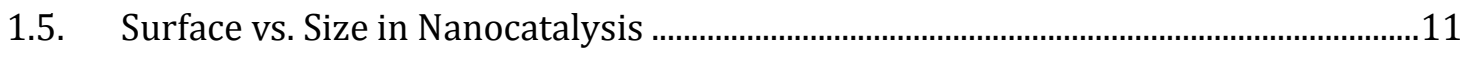

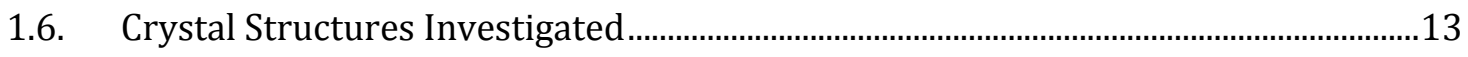

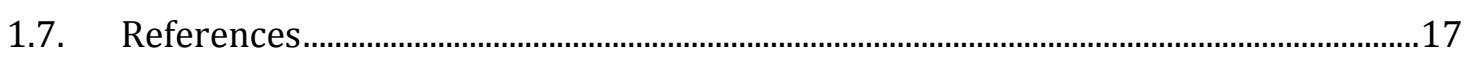

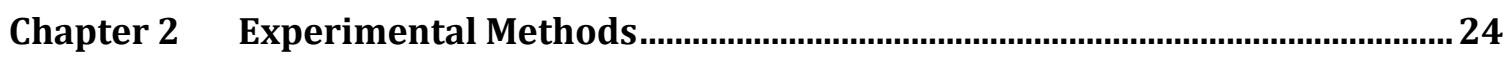

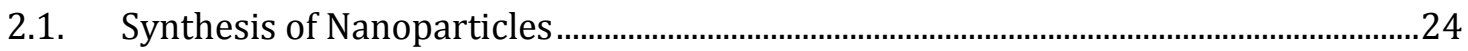

2.2. X-Ray Diffraction Techniques ………………................................................................27

2.3. Transmission Electron Microscopy (TEM) ..........................................................................32

2.4. Selected Area Electron Diffraction (SAED) .....................................................................

2.5. Energy Dispersive X-Ray Spectroscopy (EDS) ............................................................. 40

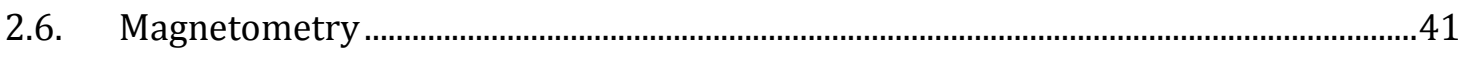

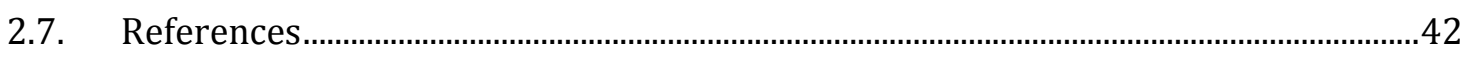

Chapter 3 Synthesis and Characterisation of Iron Nanoparticles.................................43

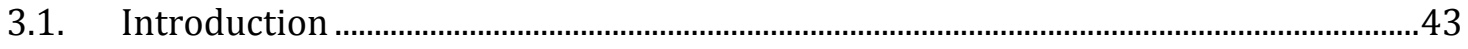

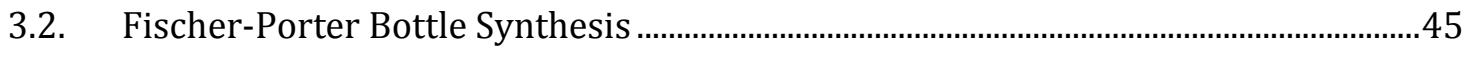

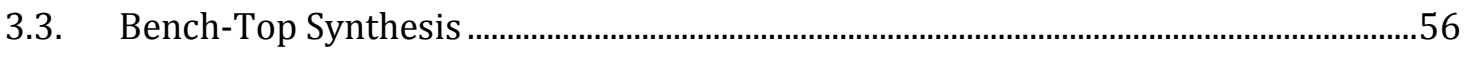

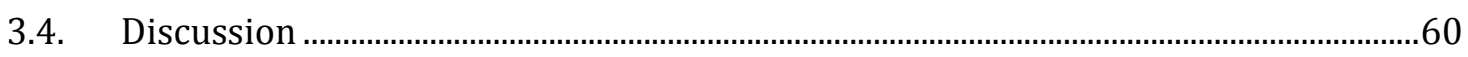

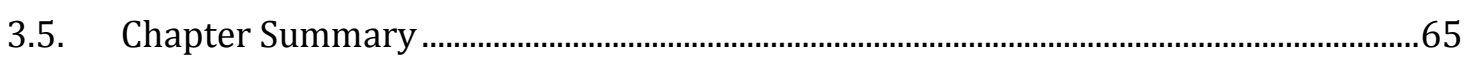

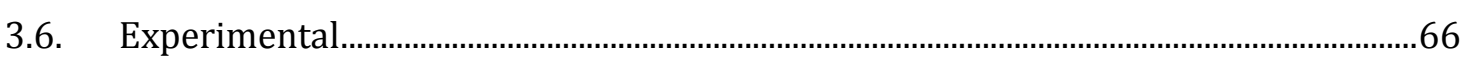

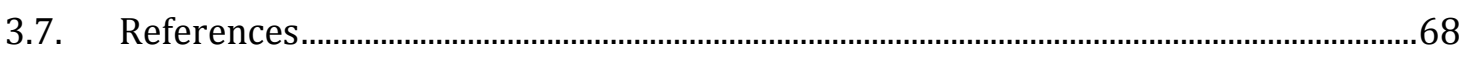


Chapter 4 Synthesis and Characterisation of Iron \& Iron Carbide Nanoparticles .. 71

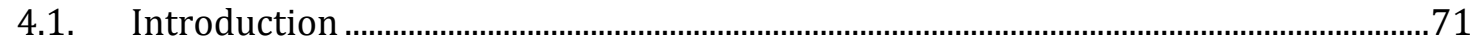

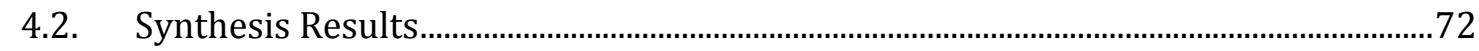

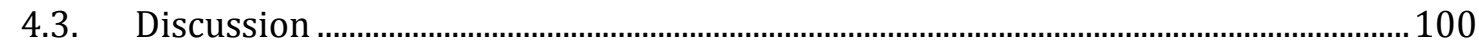

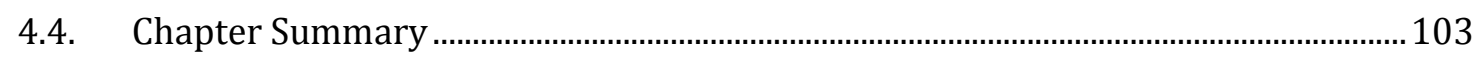

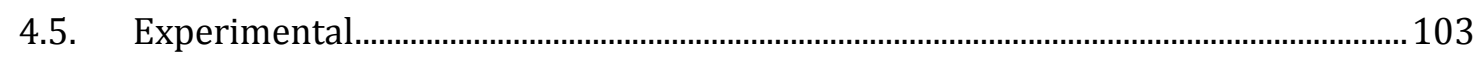

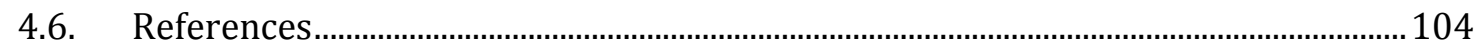

Chapter 5 Synthesis and Characterisation of Ruthenium Nanoparticles..................106

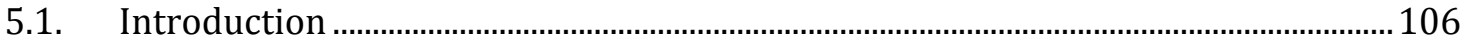

5.2. Reaction of Bis(2-Methylallyl) 1,5-Cyclooctadiene Ruthenium(II) ..........................108

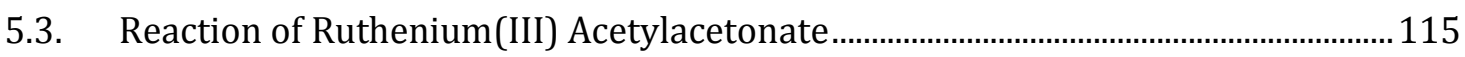

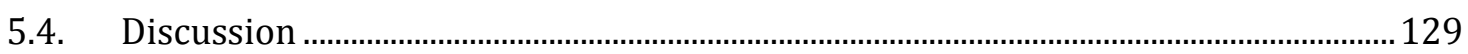

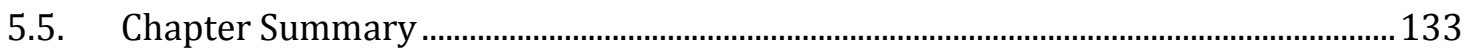

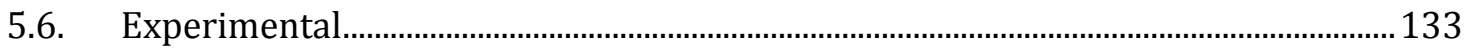

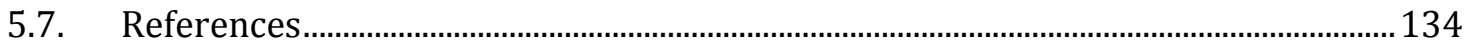

Chapter 6 Growth Study of Platinum Nanoparticles in Solution .................................137

6.1. Introduction ..............................................................................................................137

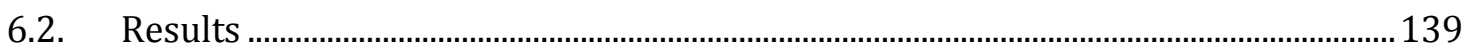

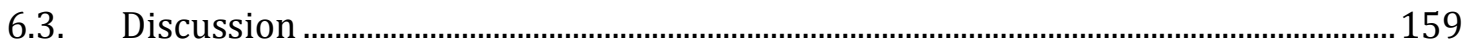

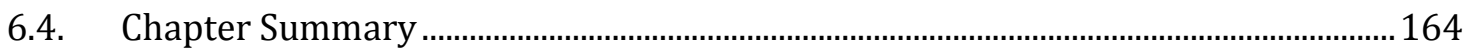

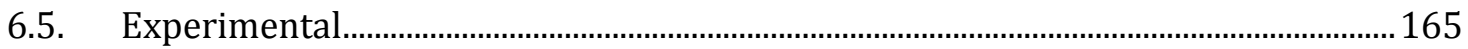

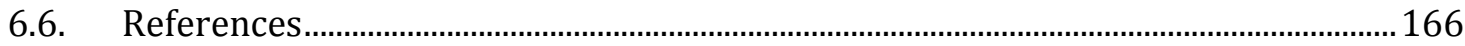

Chapter 7 Conclusions and Future Work …...............................................................169

7.1. Conclusions........................................................................................................................169

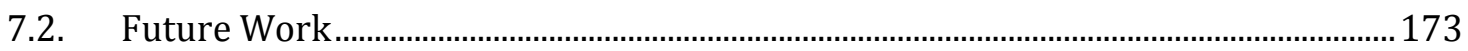

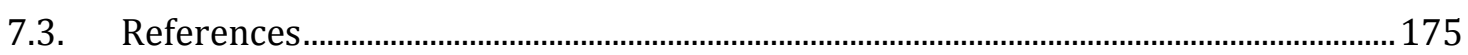

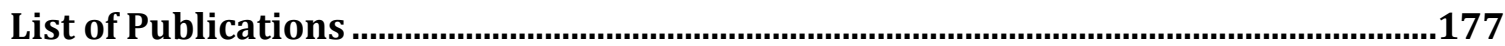




\section{List of Figures}

Figure 1-1. Nanoparticle stabilisation in solution-phase synthesis.

Figure 1-2. High-energy facets grow more quickly than low-energy facets resulting in nanocrystals of non-spherical shapes................................................................................................ 5

Figure 1-3. Selective binding of stabilising molecules leads to shape control............................. 5

Figure 1-4. Magnetisation curve of ferromagnetic and superparamagnetic materials.............. 9

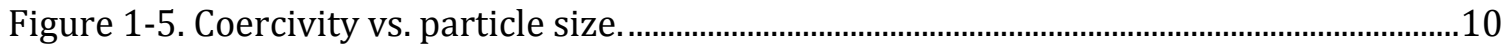

Figure 1-6. Surface-to-volume ratio (S/V) as a function of particle size.....................................12

Figure 1-7. Shell structured representation of hcp nanocrystals showing how percentage of surface atoms decreases as number of atoms increases...................................................................12

Figure 1-8. The iron-iron carbide phase diagram............................................................................15

Figure 1-9. Structure model of $\mathrm{Fe}_{3} \mathrm{C}$ (orthorhombic) .................................................................16

Figure 2-1. A Fischer-Porter bottle setup....................................................................................25

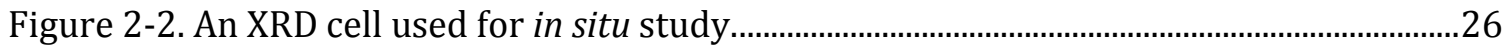

Figure 2-3. Bragg model of reflection of X-rays from a cubic crystal lattice.................................27

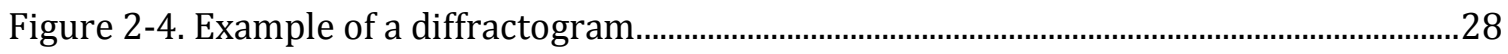

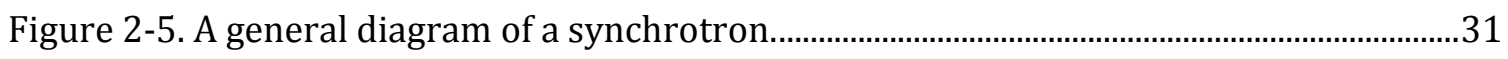

Figure 2-6. In situ XRD setup at beamline 7-2 at SSRL......................................................................32

Figure 2-7. Schematic showing the layout of the optical components in a transmission electron microscope.

Figure 2-8. Ray diagram of a transmission electron microscope in the a. diffraction and

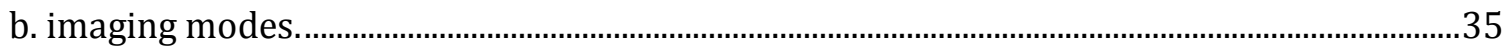

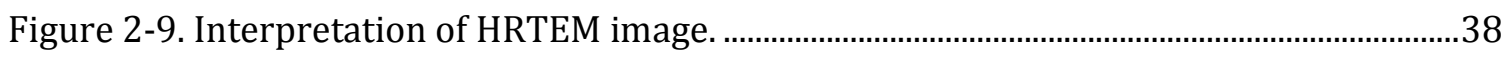

Figure 2-10. Schematic illustrates the relationship between camera length, Bragg angle, and

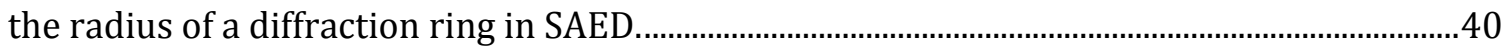

Figure 3-1. TEM and SAED results for low-concentration reaction at $130{ }^{\circ} \mathrm{C}\left(\mathrm{Fe}_{-} \mathrm{A}\right)$...............47

Figure 3-2. TEM and SAED results for middle-concentration reaction at $130^{\circ} \mathrm{C}\left(\mathrm{Fe} \_\mathrm{B}\right)$........49

Figure 3-3. Powder XRD of sample Fe_B.........................................................................................50

Figure 3-4. Iron/iron oxide core/shell nanoparticle of sample Fe_B............................................51

Figure 3-5. Magnetic properties of sample Fe_B at room temperature $(300 \mathrm{~K})$.........................52

Figure 3-6. TEM image of nanoparticles from high-concentration reaction at $130^{\circ} \mathrm{C}$ (Fe_D). 
Figure 3-7. TEM and SAED results of sample purified under a nitrogen atmosphere . .56

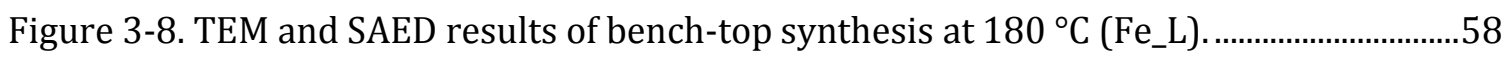

Figure 3-9. TEM and SAED results from bench-top synthesis at $160{ }^{\circ} \mathrm{C}\left(\mathrm{Fe}_{-} \mathrm{M}\right)$.........................59

Figure 3-10. A typical sponge-like nanoparticle..................................................................................60

Figure 3-11. Stability of core/shell nanoparticles of $\sim 14 \mathrm{~nm}$.....................................................63

Figure 3-12. Stability of core/shell nanoparticles of $\sim 7 \mathrm{~nm}$......................................................64

Figure 4-1. TEM and SAED results of middle-concentration reaction at $110{ }^{\circ} \mathrm{C}(\mathrm{Fe}-03)$........77

Figure 4-2. A typical iron/iron oxide core/shell nanoparticle obtained from the middle-concentration reaction at $110{ }^{\circ} \mathrm{C}(\mathrm{Fe}-03)$..

Figure 4-3. A typical iron carbide/iron oxide core/shell nanoparticle from the $0.25-\mathrm{M}$ reaction at $110^{\circ} \mathrm{C}(\mathrm{Fe}-03)$.

Figure 4-4. TEM and SAED results of middle-concentration reaction, with a 1:1 ratio of oleylamine to iron (Fe-05)

Figure 4-5. A typical core/shell nanoparticle of tetrapod structure (Fe-05).

Figure 4-6. TEM and SAED results from high-concentration reaction at $110^{\circ} \mathrm{C}(\mathrm{Fe}-06) \ldots . . . .84$

Figure 4-7. Powder XRD of nanoparticles from high-concentration reaction at $110^{\circ} \mathrm{C}$ (Fe-06).

Figure 4-8. Magnetic properties of sample Fe-06 at $300 \mathrm{~K}$.

Figure 4-9. TEM and SAED results of Expt Fe-12.

Figure 4-10. Powder XRD pattern of nanoparticles from Expt Fe-12.

Figure 4-11. TEM and SAED results of high-precursor concentration reaction with 0.2 equivalents oleylamine ( $\mathrm{Fe}-13)$

Figure 4-12. TEM and SAED results of middle-concentration solution reacted at 80 then $110{ }^{\circ} \mathrm{C}(\mathrm{Fe}-16) \ldots$

Figure 4-13. Magnetic properties of sample Fe-16 at $300 \mathrm{~K}$.

Figure 4-14. TEM and SAED results from high-concentration reaction, added with $5 \mathrm{~mL}$ excess solvent outside reaction vial (Fe-23)

Figure 4-15. HRTEM analysis of a nanodumbbell.

Figure 4-16. TEM image of nanoparticles obtained from a reaction of $\mathrm{FeCl}_{3}(\mathrm{Fe}-25)$. . .98

Figure 4-17. TEM and SAED results from reaction (Fe-25).

Figure 5-1. Ruthenium nanoparticles formed at different temperatures.

Figure 5-2. TEM images of ruthenium nanoparticles obtained from different reactions at room temperature..

Figure 5-3. TEM images of ruthenium nanoparticles obtained from different reactions at $40{ }^{\circ} \mathrm{C}$ 
Figure 5-4. Mixture of near-spherical nanoparticles and zigzag nanorods (Ru-17).............118

Figure 5-5. HRTEM image of nanoparticles observed in Expt Ru-17.

Figure 5-6. TEM image of ruthenium nanoparticles obtained from reaction of ruthenium(III) acetylacetonate under nitrogen.

Figure 5-7. TEM image of ruthenium nanoparticles obtained from reaction with low oleylamine concentration.

Figure 5-8. HRTEM analysis of a nanostick.

Figure 5-9. Nanoparticles obtained from reaction of high oleylamine to ruthenium ratio. 123

Figure 5-10. Sintering of ruthenium nanoparticles.

Figure 5-11. Worm-like nanostructures from reaction of low oleic acid:Ru ratio.

Figure 5-12. TEM image of ruthenium nanoparticles obtained from reaction of high oleic acid concentration.

Figure 5-13. HRTEM analysis of triangular plate-like and tripod nanocrystals.

Figure 5-14. Effect of temperature and amount of stabiliser on nanoparticle morphology and size.

Figure 5-15. Directions in a hexagonal lattice projected along the [001] zone axis.

Figure 6-1. In situ XRD plot for low-concentration reaction.

Figure 6-2. Shift in the calculated $d$-spacing from the bulk value for the low-concentration reaction

Figure 6-3. Normalised area and X-ray correlation length against time for the low-concentration reaction.

Figure 6-4. TEM result of the in situ experiment of low precursor concentration (Pt-1)....142

Figure 6-5. In situ XRD plot for high-concentration reaction

Figure 6-6. Shift in the calculated $d$-spacing from the bulk value for the high-concentration reaction

Figure 6-7. Normalised area and X-ray correlation length against time for the high-concentration reactions.

Figure 6-8. Time evolution of area under the $\mathrm{Pt}(111)$ peak.

Figure 6-9. TEM image of nanoparticles collected at the end of the high-concentration in situ experiment.

Figure 6-10. TEM image of platinum nanoparticles from low-concentration reaction at 80 minutes (Pt-3A).

Figure 6-11. TEM images of attached nanocrystals from low-concentration reaction at 480 minutes (Pt-3B).

Figure 6-12. TEM image of the platinum nanoparticles from low-concentration reaction at 1080 minutes (Pt-3C). 
Figure 6-13. TEM images of quasi-octapods from high-concentration reaction at 75 minutes (Pt-4A).

Figure 6-14. TEM images of etched-octapods from high-concentration reaction at 120 minutes (Pt-4B).

Figure 6-15. TEM images of porous nanocrystals from high-concentration reaction at 240 minutes (Pt-4C)...

Figure 6-16. TEM images of porous nanocrystals from high-concentration reaction at 500 minutes (Pt-4D).

Figure 6-17. TEM image of multiply-branched nanoparticle from high-concentration reaction at 900 minutes (Pt-4E).

Figure 6-18. Schematic illustration of the growth mechanism for nanoparticles in the low-concentration reaction.

Figure 6-19. Schematic illustrates the proposed growth mechanism of platinum nanocrystals in the high-concentration reaction. 


\section{List of Tables}

Table 1-1. Saturation magnetisation of iron and iron related materials......................................11

Table 1-2. Crystal structure and lattice parameters of the metals investigated.........................13

Table 1-3. Main reflections of $\alpha$-Fe...........................................................................................

Table 1-4. Main reflections of platinum. ..................................................................................... 14

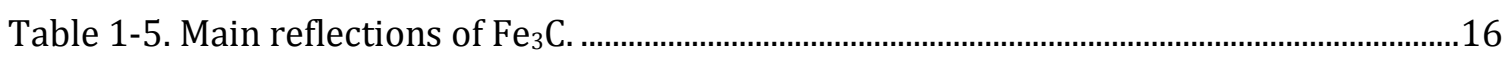

Table 3-1. Summary table of Fischer-Porter bottle synthesis. ........................................................46

Table 3-2. Index for SAED pattern in Figure 3-1b............................................................................ 47

Table 3-3. Index for the non-iron oxide diffraction rings in Figure 3-2c. ...................................50

Table 3-4. Summary table of bench-top synthesis............................................................................56

Table 3-5. Combined effect of precursor concentration and amount of oleylamine on

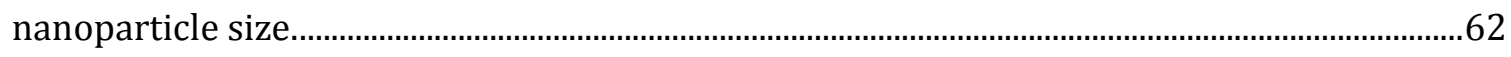

Table 3-6. Comparison of magnetic properties of similar nanoparticles.....................................65

Table 4-1. Summary table of synthesis..........................................................................................

Table 4-2. Summary table of synthesis involved the use of excess solvent. ................................74

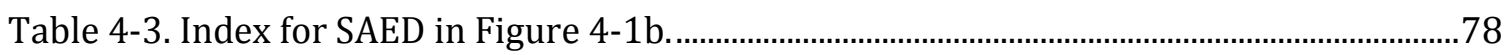

Table 4-4. Effect of precursor concentration on nanoparticle composition, morphology and

size

Table 4-5. Comparison of magnetic properties of different nanoparticles.................................103

Table 5-1. Summary table of experiment with $\mathrm{Ru}(\mathrm{MA})_{2}(\mathrm{COD})^{*}$ as the precursor..................109

Table 5-2. Reaction conditions and the corresponding observations for an experiment conducted under nitrogen atmosphere. …………......................................................................115

Table 5-3. Summary table of experiments with ruthenium(III) acetylacetonate as the precursor.

Table 5-4. Reaction conditions and the corresponding observations for Expt Ru-16

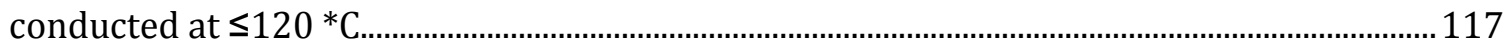

Table 6-1. Reaction conditions for off-line experiments........................................................ 147

Table 7-1. Comparison of size distribution achieved through different reactions. .................172 


\title{
Abbreviations and Synonyms
}

\author{
Abbreviations \\ acac acetylacetonate \\ bcc body-centred cubic \\ COD 1,5-cyclooctadiene \\ fcc face-centred cubic \\ fwhm full width at half maximum \\ hcp hexagonal close packed \\ HRTEM high resolution transmission electron microscopy \\ MA 2-methylallyl \\ rpm revolutions per minute \\ SAED selected area electron diffraction \\ SEM scanning electron microscopy \\ TEM transmission electron microscopy \\ XRD X-ray diffraction
}

\section{Synonyms}

oleic acid cis-9-octadecenoic acid

oleylamine cis-1-amino-9-octadecene

mesitylene 1,3,5-trimethylbenzene 


\section{Symbols and Units}

$\begin{array}{lll}\text { Symbol } & \text { Quantity } & \text { Units } \\ H & \text { applied magnetic field strength } & \text { Oe, kOe } \\ H_{C} & \text { coercivity } & \text { Oe } \\ M & \text { (mass) magnetisation } & \text { emu g-1 }^{-1} \\ M_{R} & \text { remanent magnetisation } & \text { emu g-1 }^{-1} \\ M_{S} & \text { saturation magnetisation } & \text { emu g-1 } \\ L & \text { X-ray correlation length } & \mathrm{nm}\end{array}$




\section{Chapter 1}

\section{Introduction}

\section{Chapter outline}

\subsection{General Overview}

\subsection{Solution-Phase Synthesis of Nanoparticles}

1.2.1 General Principle

1.2.2 Synthesis Method

1.3 Growth of Nanoparticles in Solution

1.3.1 Growth by Monomer Addition

1.3.2 Growth by Aggregation

1.4 Nanomagnetism

1.5 Surface vs. Size in Nanocatalysis

1.6 Crystal Structures Investigated

1.7 References

\subsection{General Overview}

The main objectives of this research project were to explore solution-phase synthesis of nanoparticles and to investigate the growth of nanoparticles in solution. The main motivation was to investigate and develop a facile synthesis route that would involve milder reaction conditions, such as lower reaction temperatures and use of less/non-toxic reagents, compared to those employed in the literature. Although solution-phase synthesis has advanced remarkably in recent years in terms of the range of materials, morphologies and size distributions achieved, knowledge about the mechanisms and kinetics governing the synthesis is relatively limited.[1-5] The present state of nanoparticle synthesis has been largely achieved empirically with some classical models for particle growth serving as 
guides.[6-8] It was thus intended, through the investigation of the synthesis itself and through in situ techniques, to investigate the growth of nanoparticles in solution.

Iron, iron carbide, ruthenium and platinum were the materials studied in this research project. Iron and iron carbide were of interest mainly because of their magnetic properties that can be exploited across many fields. ${ }^{[9]}$ Ruthenium and platinum are useful catalysts in many chemical reactions, and in the nanoscale regime the size- and shape-dependent catalytic properties have been shown to improve in various chemical processes.[10,11]

Structural analyses of the prepared nanoparticles involved the use of Transmission electron microscopy (TEM), high-resolution TEM (HRTEM), selected area electron diffraction (SAED), powder X-ray diffraction (XRD) and energy-dispersive electron spectroscopy (EDS). Scanning quantum interference device magnetometry (SQUID) was used to characterise the magnetic properties of the iron-based nanoparticles. In situ synchrotron-based XRD was employed for nanoparticle growth studies.

There are altogether 7 chapters in this thesis. The rest of this chapter provides the background information about the synthesis and properties of nanoparticles in general, with special attention paid to the materials studied in this research. Chapter 2 presents the general synthesis methods and characterisation techniques employed in this research work. Chapter 3 and 4 describe and discuss the synthesis and characterisation of iron and iron carbide nanoparticles; Chapter 3 focuses on iron and Chapter 4 on iron and iron carbide nanoparticles. In Chapter 5, the synthesis and characterisation of ruthenium nanoparticles are described and discussed. Chapter 6 presents and discusses a detailed study of the growth of platinum nanoparticles. Chapter 7 concludes the thesis with a discussion comparing and contrasting the various syntheses involved, and relating the results of syntheses to those of the growth studies. Suggested future work is presented at the end of Chapter 7.

\subsection{Solution-Phase Synthesis of Nanoparticles}

There are generally two different approaches to the synthesis of nanoparticles; the physical approach such as ball milling, and the chemical approach such as sol-gel processing.[12,13] In the chemical approach, synthesis of nanoparticles can be carried out in vapour, solution, or 
solid phase. Accounts of the various syntheses are available in many review articles published in recent years. ${ }^{[14-16]}$ In this research project, nanoparticles were prepared from a solution-phase synthesis method via a chemical route.

\subsubsection{General Principle}

The synthesis of nanoparticles in a solution via a chemical route generally involves chemical reactions of atomic or molecular species in a solution mixture followed by formation of stable nuclei with subsequent particle growth. Materials present in the reaction system typically are the reactants, solvent and stabilising agent. The reactants are the species intended to actively participate in the chemical reactions; the choice of reactants often depends on the nature of the reaction. For example, in the synthesis of metal nanoparticles by a reduction method, the reactants comprise the metal precursor and the reducing agent. Usually the non-reacting species in the solution are the solvent and the stabilising agent. The solvent acts as a medium to allow for reaction of precursor, nucleation and particle growth to occur. The stabiliser is often an organic surfactant molecule which interacts dynamically with the surface of the growing particle thus preventing nanoparticle coalescence and aggregation. Therefore, in solution phase synthesis nanoparticle size and morphology are normally influenced by factors such as supersaturation, rates of nucleation and growth processes, the type of growth process involved, and nanoparticle stabilisation.

\section{Nucleation and growth}

Synthesis begins with chemical reactions. As the reactions proceed, the solution becomes supersaturated with the reaction products in the form of a few atomic or molecular units, known as the monomers. In order to reduce supersaturation and restore the thermodynamic equilibrium state of the system, nucleation of the reaction products occurs. Stable nuclei then grow under the influence of various kinetic factors such as reaction rates of the remainder of the reactants, and diffusion rates of monomers.

In theory, very narrow size distributions can be achieved if the nuclei are formed nearly at the same time in a supersaturated solution followed by subsequent growth stages of these nuclei.[17,18] The emphasis is a brief nucleation period and the prevention of additional nucleation during the subsequent growth. As a result, the final number of particles is kept 
relatively constant from the nucleation stage through the growth stage, allowing almost all the particles to have the same growth history and therefore a small size distribution. Temporal separation of the nucleation process from the growth process is usually achieved by manipulating variables such as reaction temperature and concentration gradients.

\section{Nanoparticle stabilisation}

Nanoparticles have a large surface to volume ratio and therefore a high surface energy. Such system is thermodynamically unstable. Aggregation of the nanoparticles can easily occur due to the tendency of the system to minimise the total surface or interfacial energy, and also due to the presence of attractive van der Waals forces between the nanoparticles.

In solution phase synthesis, aggregation can be prevented by means of steric and/or electrostatic repulsion of the nanoparticles, by employing suitable stabilising agents. ${ }^{[14]}$ The stabilising molecules interact with the nanoparticles by adsorbing onto and desorbing from the nanoparticle surface dynamically, as illustrated in Figure 1-1.[19] Solution-phase synthesis that involves the use of surfactant molecules to stabilise nanoparticles is often referred to as colloidal synthesis.[20]
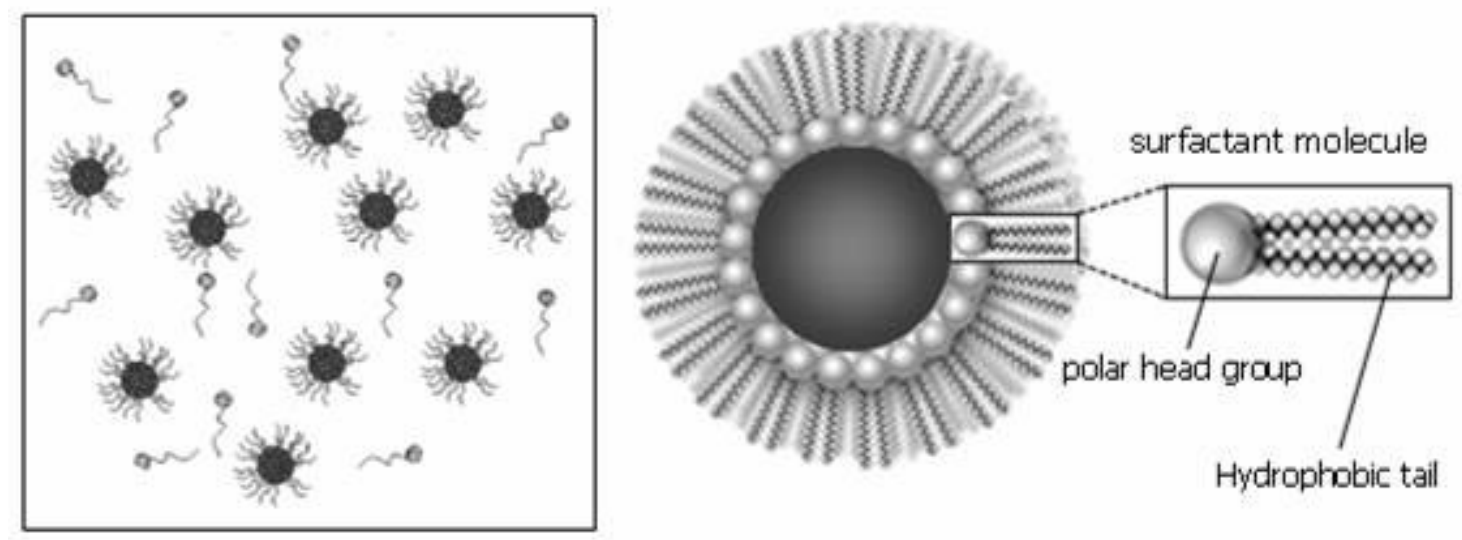

Figure 1-1. Nanoparticle stabilisation in solution-phase synthesis.

Nanoparticles are stabilised by dynamic exchange of the surfactant molecules on the nanoparticle surface, thus preventing agglomeration. On the right is a nanoparticle stabilised by organic surfactant molecules; each molecule consists of a polar head group (e.g. $\mathrm{NH}_{2}$ in oleylamine) that binds to the nanoparticle surface, and a non-polar tail that allows the nanoparticle to be suspended in an organic solvent system. 
In addition, stabilisers are sometimes used strategically to control nanoparticle morphology. Generally there are two aspects of shape control: (i) the chemical potential or stability of the different facets of a crystal, and (ii) the preferential binding of stabilising molecules onto specific crystal facets.[21,22] Non-spherical nanocrystals usually form in a kinetically controlled growth regime, as these nanocrystals are less stable than the spherical ones.[22] As the growth rate of a crystal facet varies exponentially with surface energy, high-energy facets would grow much more quickly than low-energy facets leading to nanocrystals of rod-like or disk-like shapes, as illustrated in Figure 1-2. In colloidal synthesis, the exchange of stabilising molecules on the surface of nanocrystals may vary with crystal facets. For a crystal facet that is more favourably adhered by the stabiliser, its growth rate would effectively be lowered, relative to other facets.[6,23,24] Figure 1-3[21] illustrates how shape control can be achieved by using stabilisers with preferential binding characteristics.
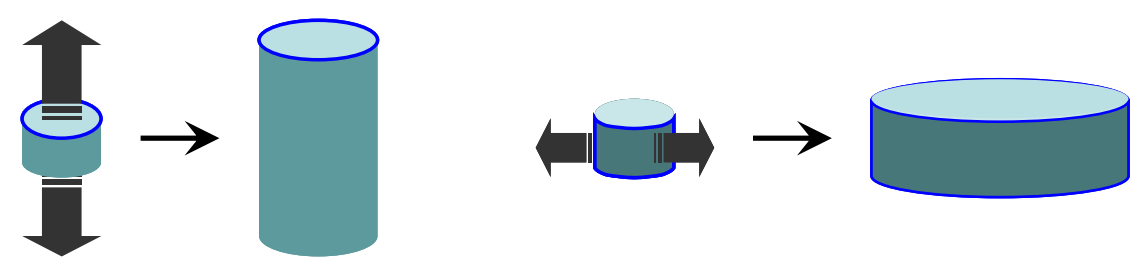

Figure 1-2. High-energy facets grow more quickly than low-energy facets resulting in nanocrystals of non-spherical shapes.
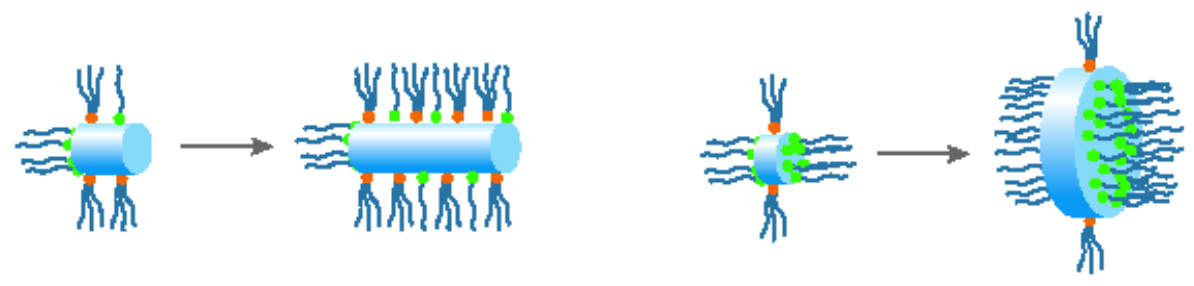

Figure 1-3. Selective binding of stabilising molecules leads to shape control.

The "red" and "green" stabilisers preferentially bind to different facets of the nanocrystal. By controlling the type and relative concentration of the stabilisers, nanoparticles of different morphologies can be obtained. 


\subsubsection{Synthesis Method}

In the literature of nanoparticle synthesis, various solution-phase synthesis methods have been reported. For metal nanoparticles, the more commonly reported solution methods are the decomposition of a metal precursor at elevated temperatures and a hydride or alcohol reduction of a metal salt in reverse micelle. Syntheses are usually carried out under an inert atmosphere.

In this project nanoparticle synthesis was carried out using Fischer-Porter bottles. According to the literature, Fischer-Porter bottles are mainly used in catalytic studies involving hydrogen or carbon monoxide. ${ }^{[25-27]}$ In terms of nanoparticle synthesis, there has been little mention of this method, apart from those reported by Chaudret and co-workers.[28-31] It was reported that with this method the nucleation and growth of nanoparticles occur at a much slower rate, compared to those involved with other more commonly adopted solution methods. ${ }^{[30,31]}$ It was proposed that the relatively slow rate of nanoparticle growth could facilitate the formation of non-symmetrical and branched nanostructures. It was thus hoped that in this project nanoparticles of unconventional morphologies be synthesised through further exploration of the Fischer-Porter bottle method.

\subsection{Growth of Nanoparticles in Solution}

As mentioned, the synthesis of nanoparticles in solution generally consists of nucleation and growth processes. It is widely accepted that temporal separation of these two processes is the requirement for achieving narrow size distribution. Nevertheless, recent reports in the literature have shown that beyond nucleation, the mechanisms involved in the growth of nanoparticles play a more significant role in determining size and size distribution, and the final morphology of the nanoparticles.[14,32,33] Generally there are two main mechanisms of nanoparticle growth in solution: (i) monomer addition and (ii) aggregation.[34-36] 


\subsubsection{Growth by Monomer Addition}

Growth by monomer addition is the classical mode of crystal growth. In this type of growth, atoms and monomers land on the surface of a growing particle and become attached. They may diffuse across the nanoparticle surface and join together to form small, two-dimensional islands and spread outward in a thin layer, with other islands forming and growing on top. The growth process is dynamic, atoms and monomers continually attach to growing particles, but often weakly adsorb and dissolve from the particle surface.

It is supposed that there is a critical nuclei size (or radius, $r_{\text {crit }}$ ) for every system under different conditions, where particles or nuclei with $r>r_{\text {crit }}$ are stable and would continue to grow and those with $r<r_{\text {crit }}$ would dissolve. The growth condition, or $r_{\text {crit, }}$, is strongly dependent on the saturation ratio, $S$, which is the ratio of $C / C_{0}$, where $C$ is the concentration of the solute and $C_{0}$ is the equilibrium concentration or solubility.[1] The relation is given by

$$
r_{c r i t}=\frac{2 V \gamma}{3 k_{B} T \ln (S)}
$$

where $V$ is the molecular volume of the precipitated species, $Y$ is the surface free energy per unit surface area, $k_{B}$ is the Boltzmann constant, and $T$ is the temperature.

Typically particles of different sizes grow at different rates. Smaller particles, particularly those slightly larger than the critical size, grow more rapidly than the larger ones because the free energy driving force is larger for smaller particles. In this case, if nucleation had stopped then all particles will eventually grow to similar sizes. This is referred to as "size focusing". A narrow size distribution can be obtained at this stage by either stopping the reaction (nucleation and growth) quickly, or by supplying more reactants to keep a saturated condition in order to maintain the $r_{\text {crit }}$ of the system.

If the growth continues and reactants are depleted due to particle growth, the saturation ratio decreases and as a consequence $r_{\text {crit }}$ increases (to $r_{\text {crit }}{ }^{\prime}$ ) according to Eq. 1.1. In this case particles with $r<r_{\text {crit }}$ ' re-dissolve into the solution, which would then be consumed by particles with $r>r_{\text {crit }}$, which in turn, would continue to grow. This process, referred to as "size defocusing", is known as the Ostwald ripening mechanism. If the reaction is stopped at this stage, the particles would usually have a bimodal size distribution. It is difficult to obtain a monodisperse sample once particle growth gets to this stage. A possible approach 
may be to extend the reaction to a much longer period of time in order to completely deplete the supersaturation and the smaller nuclei. In this case the particles become relatively large in size, often in the micrometre size regime.

\subsubsection{Growth by Aggregation}

Growth by aggregation or attachment is a mechanism where particles grow by combining with other particles when they collide in the solution. Particles grown via an aggregation route could result in a polycrystalline structure or a single crystal. In the latter case, the growth mechanism is known as the oriented attachment mechanism. ${ }^{[36,37]}$

The oriented attachment growth is a relatively new mechanism and is still not fully understood. It is proposed that when adjacent particles come together they orientate and self-assemble in a common crystallographic orientation, and fuse at the shared planar interface. The driving force for this process is thermodynamically favoured as a pair of high energy surfaces (of smaller particles) are eliminated hence the total surface free energy is greatly reduced.

As the growth condition is almost independent of the solubility product, the features of the mechanism thus contradict the conditions and parameters defined by the classical growth model. Nevertheless, it is perceived as being highly advantageous in solution-phase synthesis, mainly due to its independence of solute concentration. In recent literature, nanoparticles attached in one-, two- and three-dimensional fashions have been reported, and the mechanism has been receiving an increasing attention in both experimental and theoretical studies.

\subsection{Nanomagnetism}

Magnetic materials play many important roles in our daily life. Traditional applications of bulk magnet vary from fridge magnets, motors, power generators, to information recording and storage media. ${ }^{[38]}$ On the nanometre scale, magnetic nanoparticles have found usage in a variety of innovative applications. ${ }^{[39]}$ Magnetic nanoparticles are the active components in ferrofluids, which have long been used as liquid seals; ${ }^{[40]}$ research in using magnetic nanoparticles for high-density data storage has been going on for a decade now; ${ }^{[41,42]}$ and in 
recent years magnetic nanoparticles have emerged as a very useful material for many biological applications, and various therapeutic and diagnostic medical applications. ${ }^{[43,44]}$ Examples of the latter include biological labelling and separation, MRI contrast enhancement, and targeted drug delivery.

In order to tailor magnetic nanoparticles for a particular application, understanding magnetic properties on the nanoscale is of central importance.[45,46] Ferromagnetic materials exhibit magnetic hysteresis, which can be represented by the magnetisation curve shown in Figure 1-4. These materials retain certain magnetisation (remanence magnetisation, $M_{\mathrm{R}}$ ) after the applied field is removed. They also have high saturation magnetisation $\left(M_{S}\right)$ and coercivity $\left(H_{C}\right)$, typically in the range of $M_{S}=100-200$ emu g-1 ${ }^{-1}$ and $H_{\mathrm{C}}=300-1000$ Oe. ${ }^{[46-48]}$

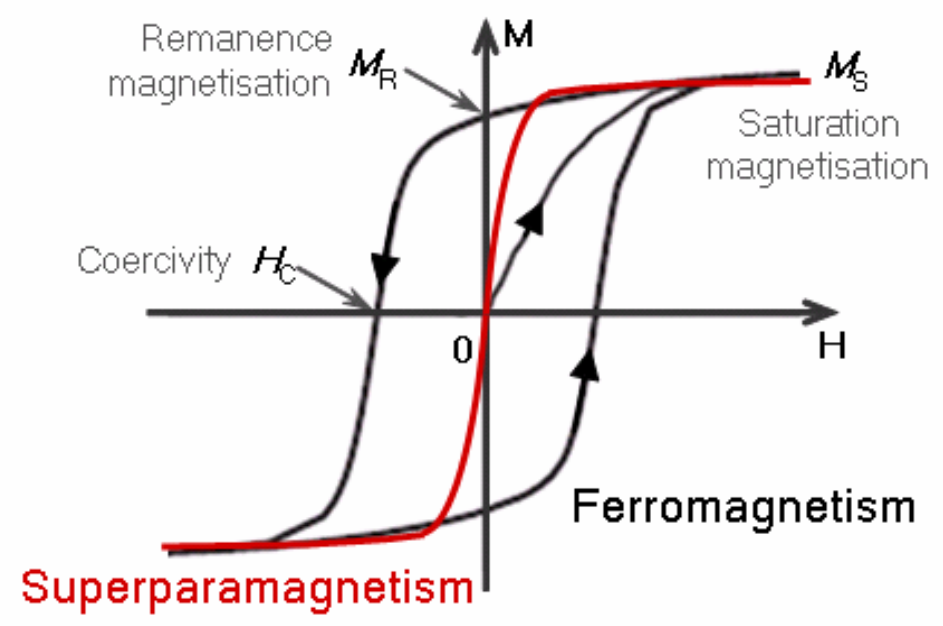

Figure 1-4. Magnetisation curve of ferromagnetic and superparamagnetic materials.

Since magnetism arises from the collective interaction of atomic magnetic dipoles, it is highly volume and temperature dependent. Figure $1-5{ }^{[49]}$ shows how $H_{\mathrm{C}}$ varies with particle size. When the size of a ferromagnet decreases to a certain critical value $D_{\mathrm{C}}$ (domain size), the particles change from a state with multiple magnetic domains to one with a single domain. A multi-domain structure is one made up of uniform magnetisation regions separated by interfaces referred to as the domain walls. Unlike each domain of a multi-domain particle that may align differently, all spins in a single-domain particle would align unidirectionally; resulting in a lower $H_{C}$ value, and zero net magnetisation in the absence of an applied field. 


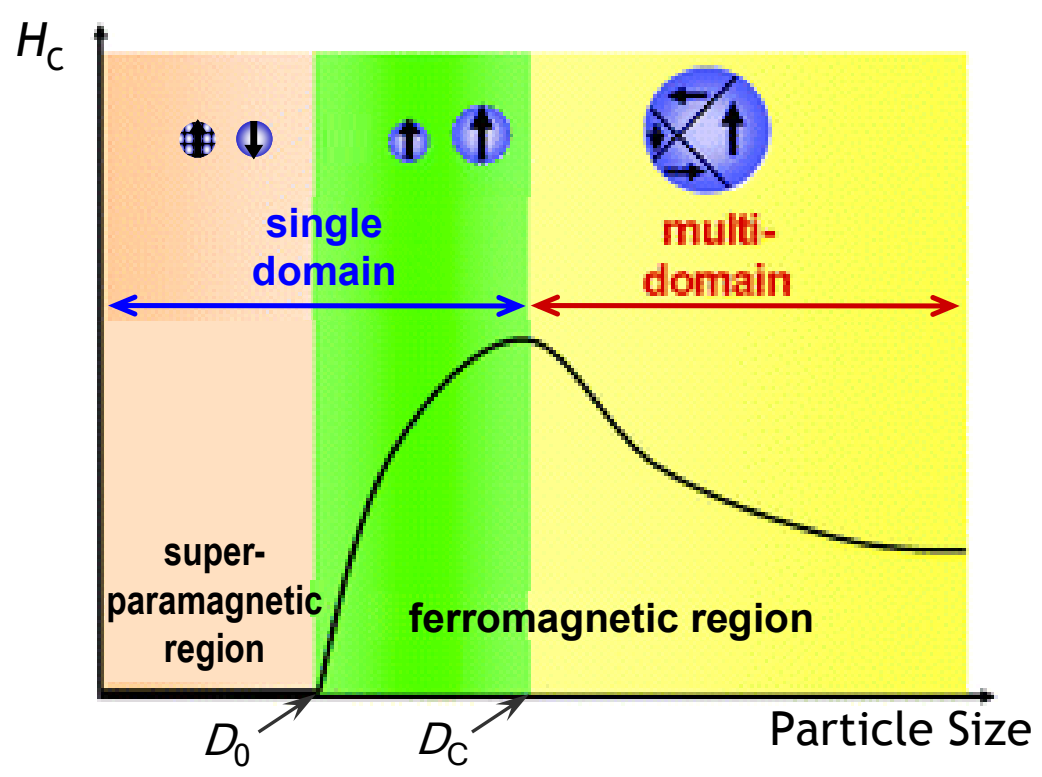

Figure 1-5. Coercivity vs. particle size.

When the particle size becomes $D_{0}$ or smaller, the $H_{\mathrm{C}}$ falls to zero and the particles do not exhibit any remanence and no coercive force is required to demagnetise the particles. This is because the relative thermal energy is large enough to allow the particles to freely reorient their spins. However, they still respond to an external magnetic field sensitively. This behaviour is referred to as superparamagnetism, which is sought for applications such as bioseparation.

As illustrated, magnetic nanoparticles can exhibit very different properties, such as the lowest (zero) and the highest coercivity observed in a material, by varying the particle size. For instance, iron is single domain at a size of $\leq 100 \mathrm{~nm}$, and is superparamagnetic at $\leq 15 \mathrm{~nm}$ (at room temperature).[46] Therefore, with the great difference in properties that occurs within tens of nanometres, magnetic materials can be tailored for different applications by tuning their size.

\section{Iron and iron carbide}

The rationale for choosing iron and iron carbide systems in this study is their magnetic properties. As mentioned, iron nanoparticles can be made ferromagnetic or superparamagnetic by varying the particle size. Besides, iron has high $M_{\mathrm{S}}$ value at room temperature (Table 1-1).[38,50] The combination of properties exhibited by iron nanoparticles makes them good candidates for various magnetic applications. However, 
development of iron nanoparticles for magnetic applications has been few compared to those of its oxides, mainly due to the susceptibility of iron to oxidation, especially at these nanodimensions where the specific surface area is much higher.[51] This has led to studies in coating the particle surface with a thin layer of shell made of materials such as gold and its own oxides.[52-54] In addition to resistance to oxidation, this coating also offers the convenience for further functionalisation for biomedical applications. ${ }^{[55,56]}$

Table 1-1. Saturation magnetisation of iron and iron related materials.

\begin{tabular}{|c|c|}
\hline Material & $\begin{array}{c}\text { Bulk } \boldsymbol{M}_{\mathbf{s}} \text { at R.T. } \\
\left(\mathbf{e m u} \mathbf{~ g}^{-\mathbf{1}} \text { ) }\right.\end{array}$ \\
\hline $\mathrm{Fe}$ & 218 \\
\hline $\mathrm{Fe} \mathrm{C}_{3}$ & 140 \\
\hline $\mathrm{Fe}_{3} \mathrm{O}_{4}$ & 82 \\
\hline$\gamma-\mathrm{Fe}_{2} \mathrm{O}_{3}$ & 70 \\
\hline
\end{tabular}

Although bulk iron carbide is generally associated with steel and its applications, iron carbide nanoparticles have found potential usage as magnetic nanoparticles. ${ }^{[57,58]}$ As shown in Table 1-1, iron carbide of the form $\mathrm{Fe}_{3} \mathrm{C}$ has higher $M_{\mathrm{S}}$ than iron oxides. Bulk $\mathrm{Fe}_{3} \mathrm{C}$ is a metallic ferromagnet at room temperature, [59] meaning $\mathrm{Fe}_{3} \mathrm{C}$ nanoparticles can be tuned to exhibit ferromagnetic or superparamagnetic behaviour by controlling the particle size. In addition, iron carbide has been reported to show higher resistance to oxidation than iron and iron nitrides. All these have made nanoparticles of iron carbide, in particular of $\mathrm{Fe}_{3} \mathrm{C}$, suitable for a diverse technological exploitation, due to their improved magnetic and mechanical properties.[60]

\subsection{Surface vs. Size in Nanocatalysis}

The surface characteristics of a nanocrystal play an important role in determining many of its properties and functions, such as solubility and catalytic activity.[61,62] This is mainly because surface-to-volume ratio (S/V) in this regime increases significantly when size decreases. For a spherical particle, the $\mathrm{S} / \mathrm{V}$ is inversely proportional to the particle radius as shown in Figure 1-6.[63] For example, a hexagonal close-packed (hcp) nanocrystal of 13 
atoms as shown in Figure 1-7[5] has a surface atom ratio of $92 \%$, compared to $76 \%$ with an additional layer of atoms on the surface.

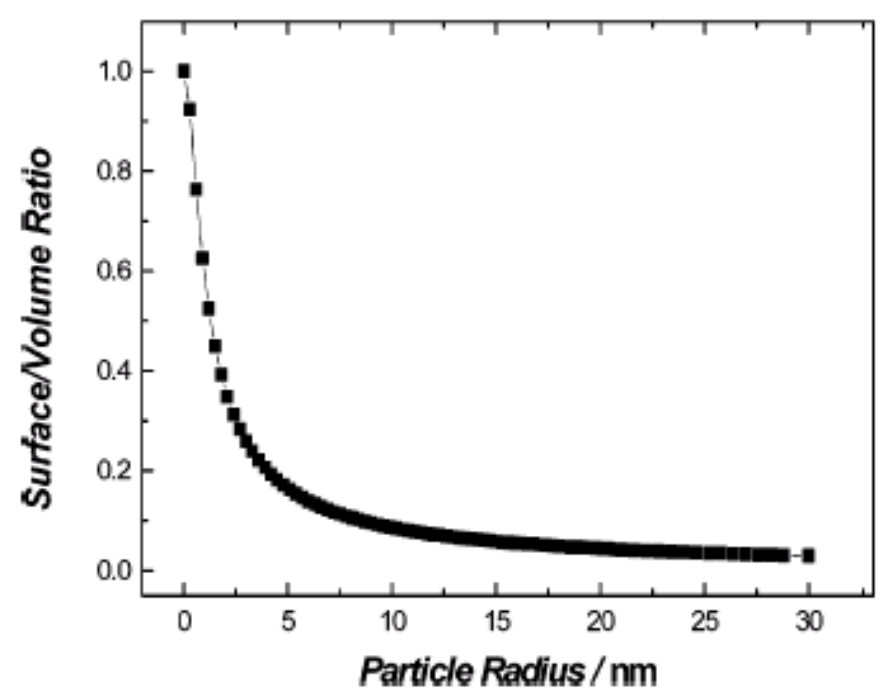

Figure 1-6. Surface-to-volume ratio (S/V) as a function of particle size.

\begin{tabular}{cccccc} 
& 88 & 858 & & \\
Number of shells & 1 & 2 & 3 & 4 & 5 \\
\hline $\begin{array}{c}\text { Number of atoms } \\
\text { in cluster }\end{array}$ & 13 & 55 & 147 & 309 & 561 \\
\hline $\begin{array}{c}\text { Percentage } \\
\text { surface atoms }\end{array}$ & $92 \%$ & $76 \%$ & $63 \%$ & $52 \%$ & $45 \%$
\end{tabular}

Figure 1-7. Shell structured representation of hcp nanocrystals showing how percentage of surface atoms decreases as number of atoms increases.

With such a system containing only a few hundred atoms, a large fraction of these atoms would be located on the surface, especially on corners and edges, as compared to a bulk or microcrystalline material..64] These atoms are coordinatively unsaturated, and have a stronger tendency to interact, adsorb, and react with other atoms or molecules in order to achieve surface stabilisation. ${ }^{[5]}$ This offers great advantage in catalysis, as the number of catalytic sites per unit mass has become much larger.

There have been numerous studies in the literature where transition metals were used to catalyse different chemical reactions.[11,65] Both ruthenium and platinum are rare transition 
metals of the platinum group metals, of which the nanoparticles are mainly exploited in the industry for their outstanding catalytic properties. Many reactions catalysed by ruthenium or platinum, or Ru-Pt alloy nanoparticles have been reported, including hydrogenation reaction, removal of organic pollutants, synthesis of low-aromatic-content diesel fuels and direct-methanol fuel cells.[66-68] Studies have also shown that these nanoparticles with a great fraction of surface atoms on edges and corners were more catalytically active than spherical and cubic ones.[69,70]

\subsection{Crystal Structures Investigated}

Crystal structures investigated in this project were iron, iron carbide, ruthenium and platinum. The crystal structure and lattice parameters of iron, ruthenium and platinum are tabulated in Table 1-2. Structural investigations of iron and platinum nanoparticles were conducted by employing synchrotron-based X-ray diffraction techniques (see Chapter 2), using a diffraction wavelength of $\lambda=0.775 \AA$. The main reflections of iron and platinum are listed in Tables 1-3 and 1-4, respectively.

Table 1-2. Crystal structure and lattice parameters of the metals investigated.

\begin{tabular}{|l|c|l|}
\hline Material & Crystal structure & Lattice parameter \\
\hline Iron, $\alpha$-Fe & bcc & $a=2.866 \AA$ \\
\hline Ruthenium & hcp & $\begin{array}{l}a=b=2.706 \AA \\
c=4.282 \AA\end{array}$ \\
\hline Platinum & fcc & $a=3.923 \AA$ \\
\hline
\end{tabular}

Table 1-3. Main reflections of $\alpha$-Fe.

\begin{tabular}{|c|c|c|c|}
\hline $\mathbf{h ~ k ~ l}$ & $\begin{array}{c}\boldsymbol{d}_{\text {hkl }} \\
(\mathbf{\AA})\end{array}$ & $\begin{array}{c}\text { Intensity } \\
\mathbf{( \% )}\end{array}$ & $\begin{array}{c}\mathbf{2 \theta} \text { at } \boldsymbol{\lambda}=\mathbf{0 . 7 7 5} \AA \\
\mathbf{(} \mathbf{})\end{array}$ \\
\hline 110 & 2.027 & 100 & 22.04 \\
\hline 200 & 1.433 & 20 & 31.37 \\
\hline 211 & 1.170 & 30 & 38.68 \\
\hline 220 & 1.013 & 10 & 44.96 \\
\hline
\end{tabular}


Table 1-4. Main reflections of platinum.

\begin{tabular}{|c|c|c|c|}
\hline $\mathbf{h ~ k ~ l}$ & $\begin{array}{c}\boldsymbol{d}_{\text {hkl }} \\
(\AA)\end{array}$ & $\begin{array}{c}\text { Intensity } \\
\text { (\%) }\end{array}$ & $\begin{array}{c}\mathbf{2 \theta} \text { at } \boldsymbol{\lambda}=\mathbf{0 . 7 7 5} \AA \\
\mathbf{(} \mathbf{~})\end{array}$ \\
\hline 111 & 2.265 & 100 & 19.70 \\
\hline 200 & 1.962 & 53 & 22.79 \\
\hline
\end{tabular}

\section{$\mathrm{Fe}_{3} \mathrm{C}$ (cementite)}

Iron carbide of numerous phases have been reported, including $\mathrm{Fe}_{2} \mathrm{C}, \mathrm{Fe}_{3} \mathrm{C}, \mathrm{Fe}_{5} \mathrm{C}_{2}$ and $\mathrm{Fe}_{7} \mathrm{C}_{3} .{ }^{[71]}$ Among the range of stoichiometries, $\mathrm{Fe}_{3} \mathrm{C}$, also known as cementite, is the most stable and in solid alloys it is stable at temperatures up to $700^{\circ} \mathrm{C}$, at which it decomposes to $\alpha$-Fe and graphite.[2] In the equilibrium binary $\mathrm{Fe}-\mathrm{C}$ phase diagram, $\mathrm{Fe}_{3} \mathrm{C}$ exists as a metastable phase, along with other stable phases $\alpha$-Fe (ferrite), $\gamma$-Fe (austenite), $\delta$-Fe and graphite. [71] This metastable phase exists because $\mathrm{Fe}_{3} \mathrm{C}$ nucleates and grows more rapidly than graphite does when supersaturated Fe-C solid solutions are aged.[73] This leads to the metastable iron-iron carbide phase diagram, as shown in Figure 1-8.[74] 


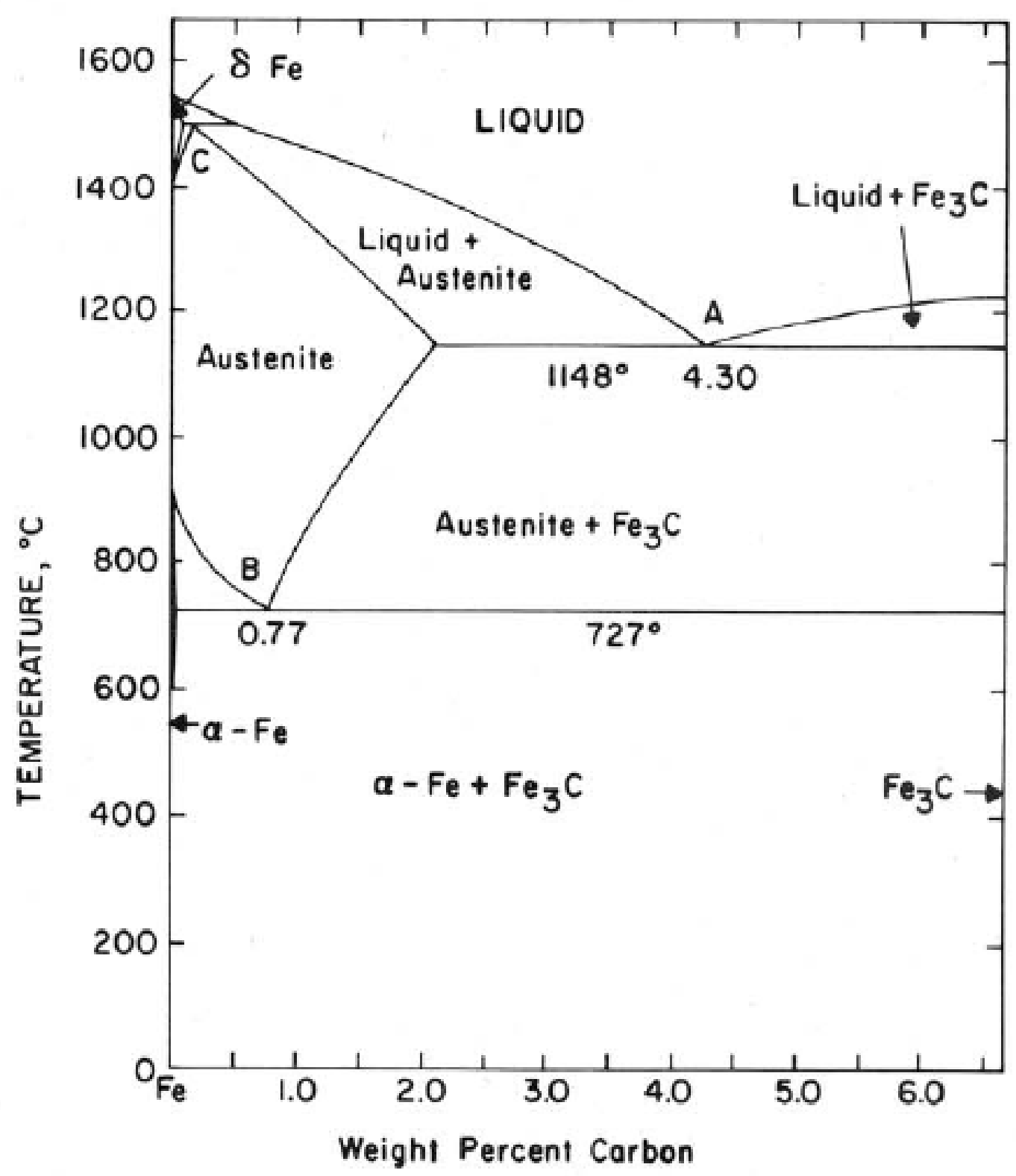

Figure 1-8. The iron-iron carbide phase diagram.

Cementite has an orthorhombic crystal structure, with approximate parameters $a=5.091 \AA$, $b=6.743 \AA, c=4.526 \AA .{ }^{[75]}$ The structure model is shown in Figure 1-9. There are 12 iron atoms and 4 carbon atoms in a unit cell, with each carbon atom surrounded by eight iron atoms and each iron atom connected to three carbon atoms. The main reflections of $\mathrm{Fe}_{3} \mathrm{C}$ are listed in Table 1-5. 

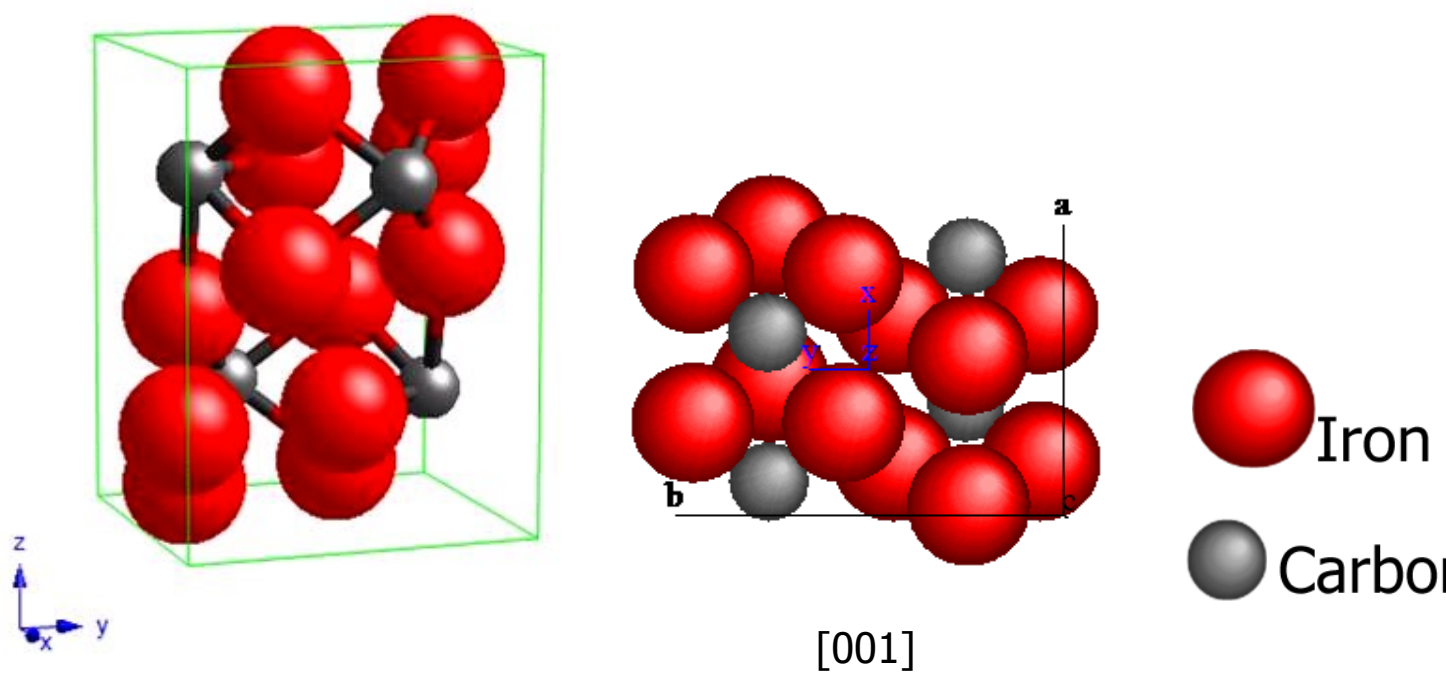

Figure 1-9. Structure model of $\mathrm{Fe}_{3} \mathrm{C}$ (orthorhombic).

Table 1-5. Main reflections of $\mathrm{Fe}_{3} \mathrm{C}$.

\begin{tabular}{|c|c|c|c|}
\hline $\mathbf{h} \mathbf{k} \mathbf{l}$ & $\begin{array}{c}\boldsymbol{d}_{\mathbf{h k l}} \\
(\mathbf{\AA})\end{array}$ & $\begin{array}{c}\text { Intensity } \\
\mathbf{( \% )}\end{array}$ & $\begin{array}{c}\mathbf{\theta} \text { at } \boldsymbol{\lambda}=\mathbf{0 . 7 7 5} \mathbf{\AA} \\
\mathbf{(} \mathbf{)}\end{array}$ \\
\hline 121 & 2.388 & 43 & 18.68 \\
\hline 210 & 2.381 & 41 & 18.73 \\
\hline 002 & 2.263 & 22 & 19.72 \\
\hline 201 & 2.218 & 22 & 20.12 \\
\hline 211 & 2.107 & 57 & 21.19 \\
\hline 102 & 2.068 & 67 & 21.60 \\
\hline 220 & 2.031 & 56 & 21.99 \\
\hline 031 & 2.013 & 100 & 22.19 \\
\hline 112 & 1.977 & 53 & 22.61 \\
\hline 131 & 1.872 & 32 & 23.89 \\
\hline 221 & 1.853 & 43 & 24.14 \\
\hline 122 & 1.763 & 19 & 25.39 \\
\hline 301 & 1.589 & 19 & 28.23 \\
\hline 123 & 1.329 & 17 & 33.89 \\
\hline 133 & 1.216 & 16 & 37.16 \\
\hline 332 & 1.162 & 20 & 38.95 \\
\hline 251 & 1.152 & 15 & 39.30 \\
\hline
\end{tabular}




\subsection{References}

[1] G. Cao, Nanostructures and Nanomaterials. Synthesis, Properties \& Applications. (Imperial College Press, London, 2004).

[2] . K. Cheetham (eds.) Nanomaterials Chemistry. (Wiley, Chichester, 2007).

[3] O. Masala and R. Seshadri, Synthesis routes for large volumes of nanoparticles. Annu. Rev. Mater. Res. 34, 41-81 (2004).

[4] C. B. Murray, C. R. Kagan and M. G. Bawendi, Synthesis and characterization of monodisperse nanocrystals and close-packed nanocrystal assemblies. Annu. Rev. Mater. Sci. 30, 545-610 (2000).

[5] J. D. Aiken III and R. G. Finke, A review of modern transition-metal nanoclusters: their synthesis, characterization, and applications in catalysis. J. Mol. Catal. A: Chem. 145, 144 (1999).

[6] V. F. Puntes, K. M. Krishnan and A. P. Alivisatos, Colloidal nanocrystal shape and size control: the case of cobalt. Science 291, 2115-2117 (2001).

[7] H. Zheng, R. K. Smith, Y.-w. Jun, C. Kisielowski, U. Dahmen and A. P. Alivisatos, Observation of single colloidal platinum nanocrystal growth trajectories. Science 324, 1309-1312 (2009).

[8] T. Sugimoto, Underlying mechanisms in size control of uniform nanoparticles. J. Colloid Interface Sci. 309, 106-118 (2007).

[9] D. C. Lee, D. K. Smith, A. T. Heitsch and B. A. Korgel, Colloidal magnetic nanocrystals: synthesis, properties and applications. Annu. Rep. Prog. Chem., Sect. C 103, 351-402 (2007).

[10] A. T. Bell, The impact of nanoscience on heterogeneous catalysis. Science 299, 16881691 (2003).

[11] A. Roucoux, J. Schulz and H. Patin, Reduced transition metal colloids: a novel family of reusable catalysts? Chem. Rev. 102, 3757-3778 (2002). 
[12] G. Schmid (ed.) Nanoparticles: From Theory to Application. (Wiley-VCH, Weinheim, 2005).

[13] D. L. Feldheim and C. A. Foss Jr. (eds.) Metal Nanoparticles. Synthesis, Characterization, and Applications. (Marcel Dekker, New York, 2002).

[14] B. L. Cushing, V. L. Kolesnichenko and C. J. O'Connor, Recent advances in the liquidphase syntheses of inorganic nanoparticles. Chem. Rev. 104, 3839-3946 (2004).

[15] J. P. Wilcoxon and B. L. Abrams, Synthesis, structure and properties of metal nanoclusters. Chem. Soc. Rev. 35, 1162-1194 (2006).

[16] J. Park, J. Joo, S. G. Kwon, Y. Jang and T. Hyeon, Synthesis of monodisperse spherical nanocrystals. Angew. Chem. Int. Ed. 46, 4630-4660 (2007).

[17] V. K. LaMer and R. H. Dinegar, Theory, production and mechanism of formation of monodispersed hydrosols. J. Am. Chem. Soc. 72, 4847-4854 (1950).

[18] J. Park, E. Lee, N.-M. Hwang, M. Kang, S. C. Kim, Y. Hwang, J.-G. Park, H.-J. Noh, J.-Y. Kim, J.-H. Park and T. Hyeon, One-nanometer-scale size-controlled synthesis of monodisperse magnetic iron oxide nanoparticles. Angew. Chem. Int. Ed. 44, 28722877 (2005).

[19] S. Nagakura, Study of metallic carbides by electron diffraction Part III. Iron carbides. J. Phys. Soc. Jpn. 14, 186-195 (1959).

[20] C. B. Murray, S. Sun, W. Gaschler, H. Doyle, T. A. Betley and C. R. Kagan, Colloidal synthesis of nanocrystals and nanocrystal superlattices. IBM J. Rev. Dev. 45, 47-56 (2001).

[21] Y. Yin and A. P. Alivisatos, Colloidal nanocrystal synthesis and the organic-inorganic interface. Nature 437, 664-670 (2005).

[22] Y.-w. Jun, J.-s. Choi and J. Cheon, Shape control of semiconductor and metal oxide nanocrystals through nonhydrolytic colloidal routes. Angew. Chem. Int. Ed. 45, 34143439 (2006).

[23] A. G. Kanaras, C. Sonnichsen, H. Liu and A. P. Alivisatos, Controlled synthesis of hyperbranched inorganic nanocrystals with rich three-dimensional structures. Nano Lett. 5, 2164-2167 (2005). 
[24] K. Philippot and B. Chaudret, Organometallic approach to the synthesis and surface reactivity of noble metal nanoparticles. C. R. Chimie 6, 1019-1034 (2003).

[25] C. A. Wesolowski and D. J. Burton, Palladium-catalyzed stereospecific carboalkoxylation of 1,2-difluoro-1-iodoalkenes and $\alpha, \beta$-difluoro- $\beta$-iodostyrenes. Tetrahedron Lett. 40, 2243-2246 (1999).

[26] S. P. Griffiths, P. Johnston and P. B. Wells, Enantioselective hydrogenation: chemistry of the modification of platinum by cinchonidine. Appl. Catal., A 191, 193-204 (2000).

[27] L. M. Rossi, J. Dupont, G. Machadoa, P. F. P. Fichtner, C. Radtke, I. J. R. Baumvol and S. R. Teixeira, Ruthenium dioxide nanoparticles in ionic liquids: synthesis, characterization and catalytic properties in hydrogenation of olefins and arenes. J. Braz. Chem. Soc. 15, 904-910 (2004).

[28] C. Pan, K. Pelzer, K. Philippot, B. Chaudret, F. Dassenoy, P. Lecante and M. J. Casanove, Ligand-stabilized ruthenium nanoparticles: synthesis, organization, and dynamics. J. Am. Chem. Soc. 123, 7584-7593 (2001).

[29] O. Margeat, D. Ciuculescu, P. Lecante, M. Respaud, C. Amiens and B. Chaudret, NiFe nanoparticles: a soft magnetic material? Small 3, 451-458 (2007).

[30] F. Dumestre, B. Chaudret, C. Amiens, M.-C. Fromen, M.-J. Casanove, P. Renaud and P. Zurcher, Shape control of thermodynamic stable cobalt nanorods through organometallic chemistry. Angew. Chem. Int. Ed. 41, 4286-4289 (2002).

[31] F. Dumestre, B. Chaudret, C. Amiens, M. Respaud, P. Fejes, P. Renaud and P. Zurcher, Unprecedented crystalline super-lattices of monodisperse cobalt nanorods. Angew. Chem. Int. Ed. 42, 5213-5216 (2003).

[32] C. B. Murray, D. J. Norris and M. G. Bawendi, Synthesis and characterization of nearly monodisperse CdE (E=S, Se, Te) semiconductor nanocrystallites. J. Am. Chem. Soc. 115, 8706-8715 (1993).

[33] Y. Xia, Y. Xiong, B. Lim and S. E. Skrabalak, Shape-controlled synthesis of metal nanocrystals: simple chemistry meets complex physics? Angew. Chem. Int. Ed. 48, 60103 (2009). 
[34] V. Privman, D. V. Goia, J. Park and E. Matijevic, Mechanism of formation of monodispersed colloids by aggregation of nanosize precursors. J. Colloid Interface Sci. 213, 36-45 (1999).

[35] M.-P. Pileni, Control of the size and shape of inorganic nanocrystals at various scales from nano to macrodomains. J. Phys. Chem. C 111, 9019-9038 (2007).

[36] Q. Zhang, S.-J. Liu and S.-H. Yu, Recent advances in oriented attachment growth and synthesis of functional materials: concept, evidence, mechanism, and future. J. Mater. Chem. 19, 191-207 (2009).

[37] J. H. Yu, J. Joo, H. M. Park, S.-I. Baik, Y. W. Kim, S. C. Kim and T. Hyeon, Synthesis of quantum-sized cubic ZnS nanorods by the oriented attachment mechanism. J. Am. Chem. Soc. 127, 5662-5670 (2005).

[38] E. P. Wohlfarth (ed.) Ferromagnetic Materials: A Handbook on The Properties of Magnetically Ordered Substances. Vol. 1. (North-Holland, Amsterdam, 1980).

[39] S. D. Bader, Opportunities in nanomagnetism. Rev. Mod. Phys. 78, 1-15 (2006).

[40] Z. Li and R. Kuldip, Ferrofluid Seal Incorporating Multiple Types of Ferrofluid (United States Patent Application Publication, US20040173972, 2004).

[41] D. Weller and A. Moser, Thermal effect limits in ultrahigh-density magnetic recording. IEEE Trans. Magn. 35, 4423-4439 (1999).

[42] R. F. Service, Is the terabit within reach? Science 314, 1868-1870 (2006).

[43] Q. A. Pankhurst, J. Connolly, S. K. Jones and J. Dobson, Applications of magnetic nanoparticles in biomedicine. J. Phys. D 36, R167-R181 (2003).

[44] P. Tartaj, M. del Puerto Morales, S. Veintemillas-Verdaguer, T. González-Carreno and C. J. Sema, The preparation of magnetic nanoparticles for applications in biomedicine. J. Phys. D 36, R182-R197 (2003).

[45] X. Batlle and A. Labarta, Finite-size effects in fine particles: magnetic and transport properties. J. Phys. D 35, R15-R42 (2002).

[46] R. H. Kodama, Magnetic nanoparticles. J. Magn, Magn. Mater. 200, 359-372 (1999). 
[47] S. Sun, Recent advances in chemical synthesis, self-assembly, and applications of FePt nanoparticles. Adv. Mater. 18, 393-403 (2006).

[48] A.-H. Lu, E L. Salabas and F. Schüth, Magnetic nanoparticles: Synthesis, protection, functionalization, and application. Angew. Chem. Int. Ed. 46, 1222-1244 (2007).

[49] Y.-W. Jun, J.-W. Seo and J. Cheon, Nanoscaling laws of magnetic nanoparticles and their applicabilities in biomedical sciences. Acc. Chem. Res. 41, 179-189 (2008).

[50] Y. Shiratsuchi, M. Yamamoto and S. D. Bader, Magnetism and surface structure of atomically controlled ultrathin metal films. Prog. Surf. Sci. 82, 121-160 (2007).

[51] L. Signorini, L. Pasquini, L. Savini, R. Carboni, F. Boscherini, E. Bonetti, A. Giglia, M. Pedio, N. Mahne and S. Nannarone, Size-dependent oxidation in iron/iron oxide coreshell nanoparticles. Phys. Rev. B 68, 195423-195423 (2003).

[52] E. E. Carpenter, S. Calvin, R. M. Stroud and V. G. Harris, Passivated iron as core-shell nanoparticles. Chem. Mater. 15, 3245-3246 (2003).

[53] Y. Qiang, J. Anthony, A. Sharma, J. Nutting, D. Sikes and D. Meyer, Iron/iron oxide coreshell nanoclusters for biomedical applications. J. Nanopart. Res. 8, 489-496 (2006).

[54] A. Shavel, B. Rodríguez-González, M. Spasova, M. Farle and L. M. Liz-Marzán, Synthesis and characterization of iron/iron oxide core/shell nanocubes. Adv. Funct. Mater. 17, 3870-3876 (2007).

[55] A. K. Gupta and M. Gupta, Synthesis and surface engineering of iron oxide nanoparticles for biomedical applications. Biomater. 26, 3995-4021 (2005).

[56] S. Laurent, D. Forge, M. Port, A. Roch, C. Robic, L. V. Elst and R. N. Muller, Magnetic iron oxide nanoparticles: synthesis, stabilization, vectorization, physicochemical characterizations, and biological applications. Chem. Rev. 108, 2064-2110 (2008).

[57] Nanocrystalline $\alpha$ - $\mathrm{Fe}, \mathrm{Fe}_{3} \mathrm{C}$, and $\mathrm{Fe}_{7} \mathrm{C}_{3}$ produced by $\mathrm{CO}_{2}$ laser pyrolysis. J. Mater. Res. 8, 1666-1674 (1993).

[58] X. Q. Zhao, Y. Liang, Z. Q. Hu and B. X. Liu, Oxidation characteristics and magnetic properties of iron carbide and iron ultrafine particles. J. Appl. Phys. 80, 5857-5860 (1996). 
[59] A. Tsuzuki, S. Sago, S.-I. Hirano and S. Naka, High temperature and pressure preparation and properties of iron carbides $\mathrm{Fe}_{7} \mathrm{C}_{3}$ and $\mathrm{Fe}_{3}$ C. J. Mater. Sci. 19, 25132518 (1984).

[60] T. J. Goodwin, S. H. Yoo, P. Matteazzi and J. R. Groza, Cementite-iron nanocompositie. Nanostruct. Mater. 8, 559-566 (1997).

[61] G. Diaz and M. Jose-Yacaman, Advanced characterization of nanoparticles: experimental evidence of surface roughness. Mater. Chem. Phys. 41, 240-244 (1995).

[62] A. P. Alivisatos, Nanocrystals: Building blocks for modern materials design. Endeavour 21, 56-60 (1997).

[63] C. Burda, X. Chen, R. Narayanan and M. A. El-Sayed, Chemistry and properties of nanocrystals of different shapes. Chem. Rev. 105, 1025-1102 (2005).

[64] A. P. Alivisatos, Perspectives on the physical chemistry of semiconductor nanocrystals. J. Phys. Chem. 100, 13226-13239 (1996).

[65] R. Narayanan and M. A. El-Sayed, Catalysis with transition metal nanoparticles in colloidal solution: Nanoparticle shape dependence and stability. J. Phys. Chem. B 109, 12663-12676 (2005).

[66] J. Ning, J. Xu, J. Liu and F. Lu, Selective hydrogenation of benzene to cyclohexene over colloidal ruthenium catalyst stabilized by silica. Catal. Lett. 109, 175-180 (2006).

[67] L. Pino, V. Recupero, S. Beninati, A. K. Shukla, M. S. Hegde and P. Bera, Catalytic partial-oxidation of methane on a ceria-supported platinum catalyst for application in fuel cell electric vehicles. Appl. Catal., A 225, 63-75 (2002).

[68] U. A. Paulus, U. Endruschat, G. J. Feldmeyer, T. J. Schmidt, H. Bonnemann and R. J. Behm, New PtRu alloy colloids as precursors for fuel cell catalysts. J. Catal. 195, 383393 (2000).

[69] R. Narayanan and M. A. El-Sayed, Changing catalytic activity during colloidal platinum nanocatalysis due to shape changes: electron-transfer reaction. J. Am. Chem. Soc. 126, 7194-7195 (2004). 
[70] A. Miyazaki, I. Balint, K.-i. Aika and Y. Nakano, Preparation of Ru nanoparticles supported on $\gamma \mathrm{Al}_{2} \mathrm{O}_{3}$ and its novel catalytic activity for ammonia synthesis. J. Catal. 204, 364-371 (2001).

[71] T. B. Massalski, H. Okamoto, P. R. Subramanian and L. Kacprzak (eds.) Binary Alloy Phase Diagrams, 2nd ed. (ASM International, Ohio, U.S.A., 2001).

[72] W. Lengauer and A. Eder, "Carbides: transition metal solid-state chemistry" in Encyclopedia of Inorganic Chemistry, edited by R. B. King (Wiley, 2006).

[73] H. K. D. H. Bhadeshia and R. W. K. Honeycombe, Steels. Microstructure and Properties, 3rd ed. (Elsevier, Oxford, U.K., 2006).

[74] G. J. Long and H. P. Leighly Jr., The iron-iron carbide phase diagram. J. Chem. Educ. 59, 948-953 (1982).

[75] Natl. Bur. Stand. (U.S.) Monogr. 25,21,72, ICDD 00-035-0772 (1985). 


\section{Chapter 2}

\section{Experimental Methods}

\section{Chapter outline}

2.1 Synthesis of Nanoparticles

2.1.1 Fischer-Porter Bottle

2.1.2 X-Ray Diffraction Cell

2.2 X-Ray Diffraction Techniques

2.2.1 Synchrotron-Based XRD

2.3 Transmission Electron Microscopy (TEM)

2.3.1 High-Resolution TEM (HRTEM) Imaging

2.4 Selected Area Electron Diffraction (SAED)

2.5 Energy Dispersive X-Ray Spectroscopy (EDS)

2.6 Magnetometry

2.7 References

\subsection{Synthesis of Nanoparticles}

This section describes the general synthetic procedure employed in this study. Detailed experimental procedures for synthesising the different types of nanoparticles are described in the experimental sections of the respective results chapters.

\subsubsection{Fischer-Porter Bottle}

A Fischer-Porter bottle is a type of pressure reaction vessel, in which reaction takes place in an enclosed atmosphere of some gas of choice. Figure 2-1 shows a typical Fischer-Porter bottle setup used in this study. 
A vial containing the reaction mixture was first placed inside the Fischer-Porter bottle. In a typical experiment, the reaction mixture comprised a metal precursor (e.g. ruthenium(III) acetylacetonate), a solvent (e.g. toluene), and a stabilising agent (e.g. oleylamine).

The Fischer-Porter bottle was fitted with a two-valve adapter, which allowed the regulation of the atmosphere content within the bottle. One of the valves was connected to a vacuum pump and the other usually to a hydrogen cylinder. Using the valve adapter the bottle was purged at least 3 times and filled with a gas of choice (usually hydrogen gas), often up to a pressure in the range of 100-300 kPa, and sealed. The Fischer-Porter bottle was then placed into an oven heated at a reaction temperature in the range of $40-160{ }^{\circ} \mathrm{C}$ for nanoparticle synthesis.

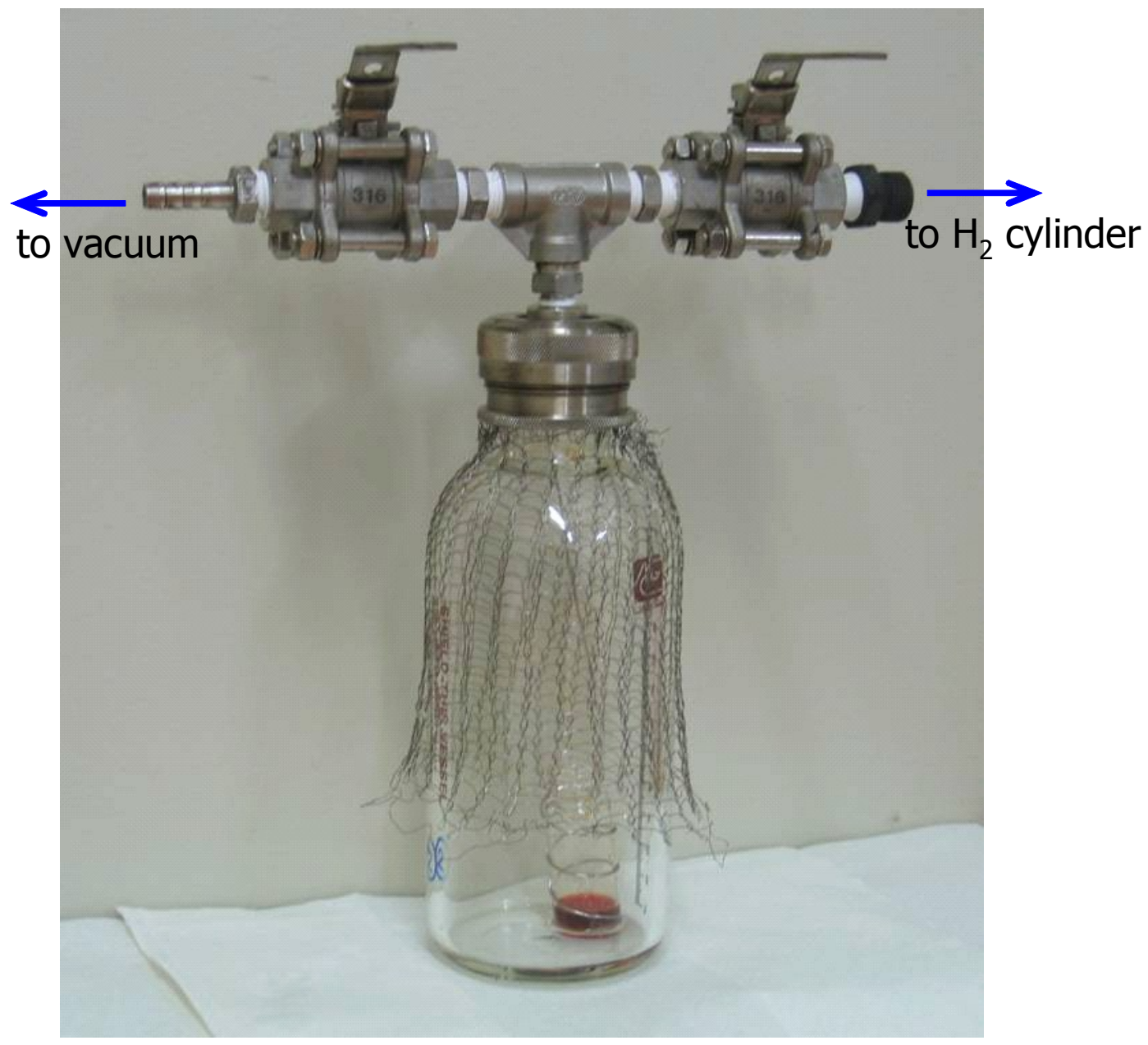

Figure 2-1. A Fischer-Porter bottle setup.

The vial containing the reaction mixture was secured in the Fischer-Porter bottle using a stainless steel wire. The Fischer-Porter was protected with a wire mesh shield, in case of implosion or explosion. 


\subsubsection{X-Ray Diffraction Cell}

The growth study of platinum nanoparticles was conducted using in situ synchrotron-based X-ray diffraction (XRD) techniques. An XRD cell suitable for in situ study at a synchrotron beamline was custom-made. The XRD cell was made of stainless steel and comprised two parts mounted together by screws. A photo of the cell is shown in Figure 2-2.

The bottom piece was the reaction vessel where reactions took place, whereas the top was a cylindrical chamber equipped with a three-way valve to allow for regulation of reaction atmosphere. The two pieces together gave the reaction cell an internal volume of about $300 \mathrm{~mm}^{3}$. The disk-shaped reaction vessel was $1 \mathrm{~mm}$ thick, with polyimide (Kapton) windows on both flat faces. It had an opening on the top to allow for transfer of fluids. This was also where the top piece was mounted.

In a typical experiment, about $0.05 \mathrm{~mL}$ of reaction mixture was transferred into the reaction vessel using a micro syringe and needle. The cell was closed by mounting the reaction vessel and the valve chamber together. Using the valve, the cell was flushed through with hydrogen gas, before being filled to $200 \mathrm{kPa}$ hydrogen, and sealed.

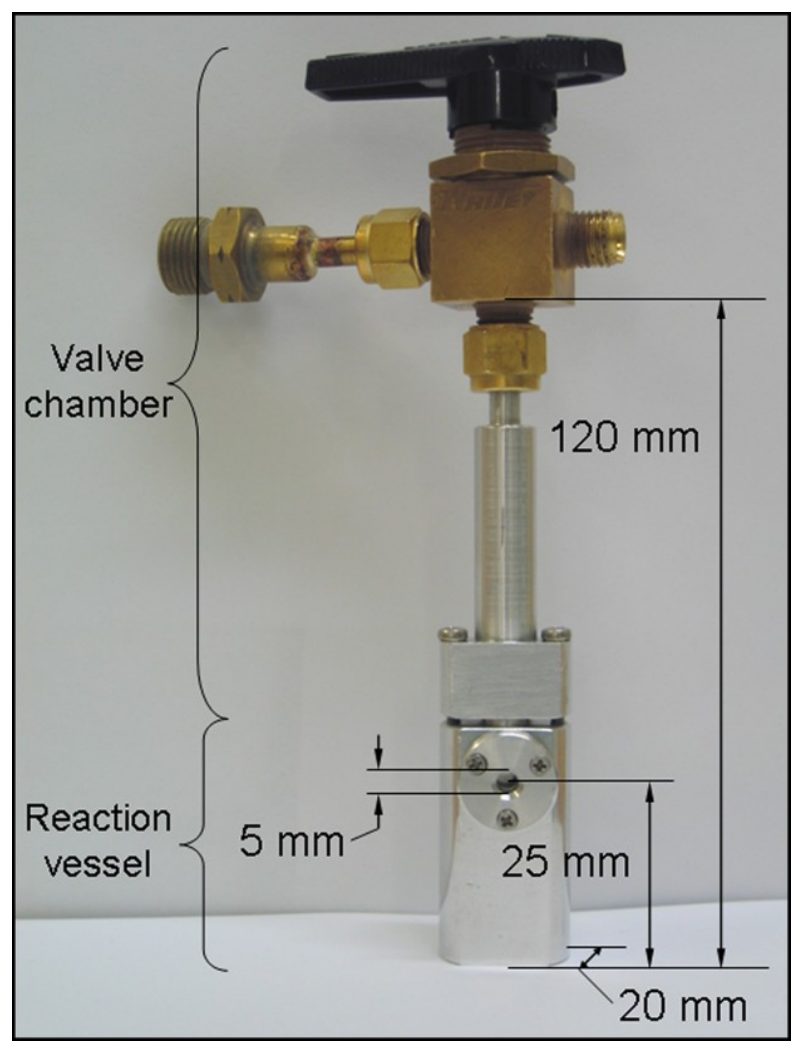

Figure 2-2. An XRD cell used for in situ study. 


\subsection{X-Ray Diffraction Techniques}

X-ray diffraction (XRD) techniques are useful for revealing structural information of crystalline materials, as these materials cause deflections when illuminated with X-ray. Comprehensive information of the basic principles of the technique and its use for structural analysis can be found in Ladd and Palmer, [1] and Giacovazzo.[2]

\section{Theoretical basis}

X-rays are electromagnetic waves whose wavelengths are in the range of $\sim 0.1-100 \AA$, and can be scattered by interaction with matter, primarily through the matter's electrons.[1] The radiation is scattered in all directions for a given scattering angle. As crystals are a three-dimensional array of repeating units, a regular array of scattered waves is produced. The constructive interference of the waves then give rise to a diffraction pattern consisting of specific directions. This condition is only satisfied for a given interplanar spacing $d_{\mathrm{hkl}}$. Figure 2-3 illustrates the Bragg model of reflection of X-rays from a set of parallel planes. The angle between the incident and the scattered beam, called the diffraction angle, $2 \theta$, can be measured experimentally. If radiation source of known wavelength is used, $d_{\mathrm{hkl}}$ can be determined from the Bragg equation:

$$
\left.2 d_{h k l} \sin \theta=n \lambda \quad \text { (Eq. 2.1 }\right)^{[1]}
$$

where $n$ is the diffraction order (conventionally assumed to be 1 ), and $\lambda$ is the diffraction wavelength.

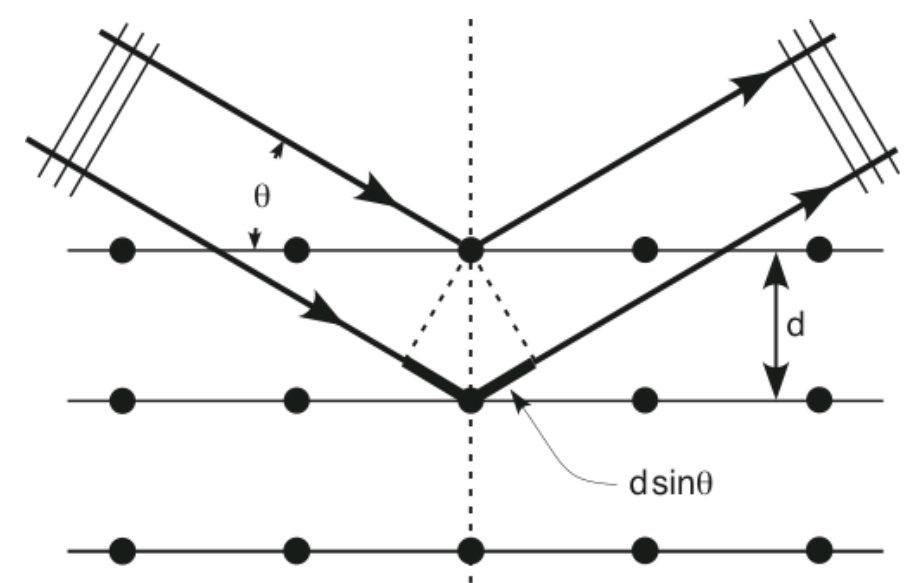

Figure 2-3. Bragg model of reflection of X-rays from a cubic crystal lattice. 
For a single crystal with a given orientation to the incident X-ray beam, only a few reflections may result, as the set of planes with interplanar spacing satisfying the Bragg condition is limited. In order to observe all possible reflections, the orientation of the crystal with respect to the incident beam must be systematically varied to bring all planes sequentially into diffraction conditions.

In powder XRD, the sample consists of many small, randomly-oriented crystallites. As one or more crystals will be in the orientation to give the correct Bragg angle, the Bragg condition for all interplanar spacings will be satisfied, and reflections from all the crystallites can be observed simultaneously. The diffracted X-rays are usually displayed as a line graph of intensity versus $2 \theta$, as shown in Figure 2-4.[3]

The absolute intensity of X-ray reflections is dependent on experimental parameters such as the intensity of the X-ray source, geometry of the diffractometer, integration time and the degree of adsorption of the sample. The intensity of the reflections is usually normalised and reported as a relative intensity, which is given by:

$$
\left.F(h k l)=\sum_{j=1}^{n} f_{j, \theta} \exp \left[i 2 \pi\left(h x_{j}+k y_{j}+l z_{j}\right)\right] \quad \text { (Eq. 2.2 }\right)^{[\mathbf{1}]}
$$

where $F(h k l)$, the structure factor of a set of planes with indices $h k l$, is the sum of scattering contributions from all $n$ atoms within the unit cell. The contribution of the $j$ th atom is the product of its atomic scattering factor $f_{j, \theta}$, and an exponential term containing its fractional coordinates within the unit cell.

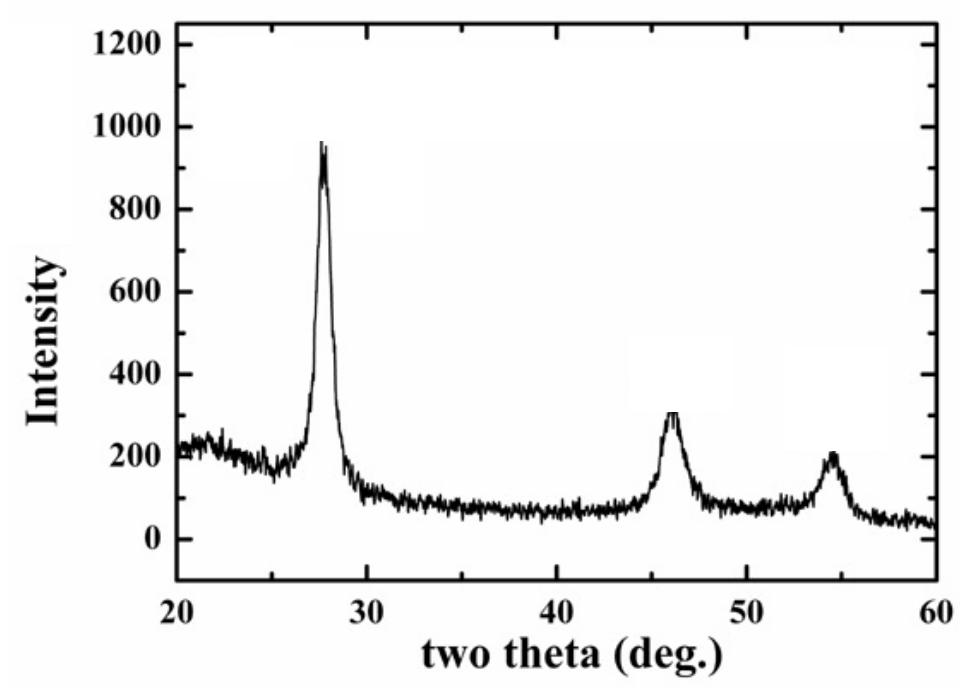

Figure 2-4. Example of a diffractogram.

Each peak represents a position where the X-ray has been diffracted by the crystal lattice. 


\section{Sample preparation and data interpretation}

Samples for powder XRD measurement were prepared by dropping the nanoparticle suspension onto a piece of $\mathrm{Si}$ wafer, and the solvent was allowed to evaporate under ambient conditions.

Crystal structures were identified by comparing the XRD pattern against a database maintained by the International Centre for Diffraction Data (ICDD). The intensity profile of the XRD peaks could be used to gain information about the crystalline structures within the sample. The area under the peak is proportional to the relative abundance of the corresponding crystalline phase in a sample, especially one with a mixture of phases. The average crystallite size can be estimated from the full width at half maximum (fwhm) of the respective peak.

In this project, the area under the peaks and the fwhm of the peaks were obtained by fitting the diffraction peaks to Lorentzian line profiles, using Origin v7.5. Information on the crystallite size was obtained by calculating the X-ray correlation length $(L)$ from the fwhm of the XRD peak using the Scherrer equation:

$L=\frac{0.9 \lambda}{B \cos \theta} \quad$ (Eq. 2.3) ${ }^{[4]}$

where $\lambda$ is the wavelength of the $\mathrm{X}$-ray radiation, $B$ is the fwhm of the peak, and $\theta$ is the angle of diffraction

Often, the diameter of a spherical crystallite is estimated to be $4 /{ }_{3} L$. This assumes that the crystallites are all perfect, spherical and of approximately uniform size, which is seldom the case in practical situation. ${ }^{44,5]}$ Other factors such as structural defects and variations in crystal composition can also lead to line broadening. Nevertheless, when used in conjunction with other techniques such as TEM and SAED, the results are able to provide an accurate overall picture of the entire sample. 


\subsubsection{Synchrotron-Based XRD}

Synchrotron radiation was used as a source for powder XRD measurements and in situ XRD experiments in this project. Measurements were conducted at the Stanford Synchrotron Radiation Lightsource (SSRL) at beamline 7-2.

Synchrotron radiation is an electromagnetic radiation generated when electrons of very high energy, travelling at virtually the speed of light, are forced to change direction under the influence of a magnetic field. A general diagram of a synchrotron is shown in Figure 2-5.[6] The electrons are circulated around a storage ring by a series of magnets. At the Stanford Synchrotron Radiation Lightsource (SSRL), electrons are accelerated up to $3 \mathrm{GeV}$, and the storage ring has a circumference of $234 \mathrm{~m} .{ }^{[7]}$ This radiation, 10000 times as intense as the solar radiation, ranges in wavelengths from infrared through to X-rays. The extremely intense X-rays can be used to investigate various forms of matter ranging from objects of atomic and molecular size to bulk materials. A comprehensive background, description, and applications of synchrotron radiation can be found in Wiedemann.[8]

There are considerable advantages in using synchrotron radiation for XRD measurement. Synchrotron radiation is extremely intense and highly collimated, which offers exceptional resolution and signal-to-noise ratio. This enables small crystallites of materials with very close $d$-spacings, such as $\alpha$-Fe and $\mathrm{Fe}_{3} \mathrm{C}$, to be determined with much better accuracy. Besides, synchrotron-based XRD can be conducted with very small sample size without sacrificing signal quality; whereas for conventional powder XRD, relatively large amounts of sample have to be prepared. Synchrotron-based XRD was used in this project in place of powder XRD. While electron diffraction (see Section 2.4) was used extensively in this project to provide localised information, the powder XRD technique was used to obtain structural confirmation of an entire sample.

Another advantage of synchrotron-based XRD over conventional XRD is the much shorter pulses emitted, which enables the recording of a diffractogram in a few minutes with sufficient count rates, which cannot be achieved with a standard laboratory diffractometer. This unique feature was exploited for time-resolved, in situ studies of the growth of platinum nanoparticles. Evaluation of the diffractograms collected continuously through the course of reaction could provide indications for changes in the crystallinity, nanocrystal 
concentration and crystallite size of the sample in real time, which could eventually lead to deductions for growth kinetics.

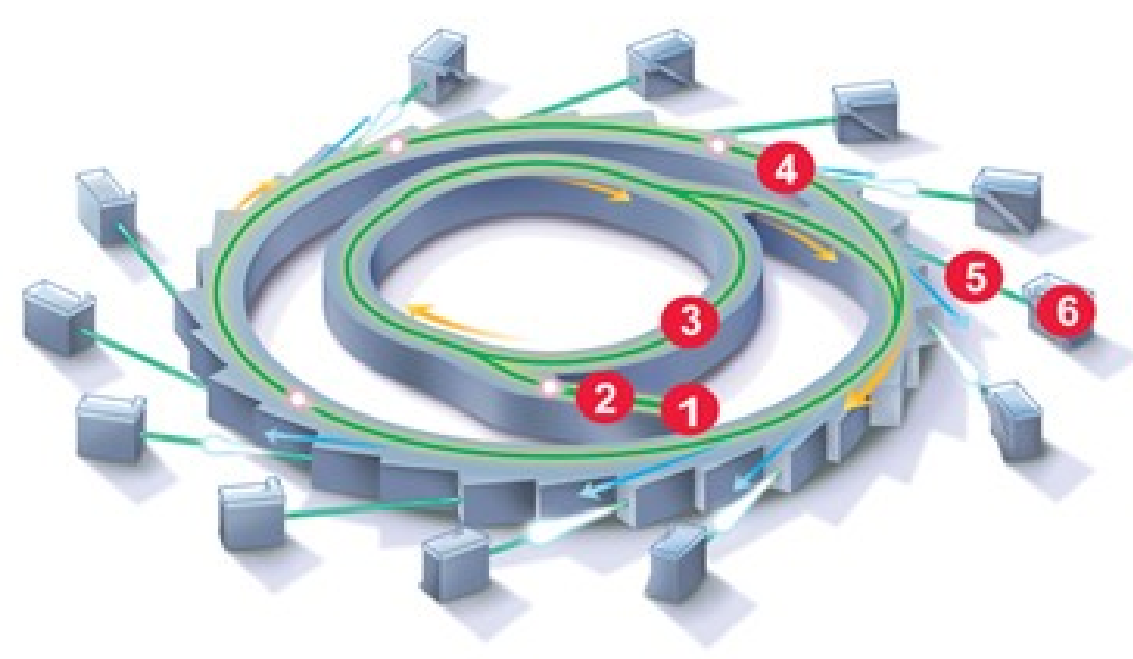

\section{Figure 2-5. A general diagram of a synchrotron.}

Electrons are produced at the electron gun (1), and accelerated by the linear accelerator (2) to virtually the speed of light. The energy of the electron beam is further increased (to $3 \mathrm{GeV}$ at SSRL) at the booster ring (3), after which the electrons are stored in the storage ring (4) before being channelled to individual beamlines (5). Beamlines are designed to select appropriate wavelengths for particular research techniques, which are used in different experiments conducted the end-stations (6).

\section{In situ XRD}

Figure 2-6 shows the setup at beamline 7-2 at SSRL for the in situ experiments.

At the start of an experiment, the reaction cell was inserted into a ceramic heating block fitted with a temperature controller and a thermocouple. It usually took less than 5 minutes to heat the ceramic block from room temperature to $70{ }^{\circ} \mathrm{C}$, which was the temperature maintained for the duration of the experiment. The XRD measurements were conducted using radiation of $\lambda=0.775 \AA$. Diffractograms were collected over the $\operatorname{Pt}(111)$ and $\operatorname{Pt}(200)$ peaks between $2 \theta$ values of 18 and $24^{\circ}$, using step scans of $0.05^{\circ}$ /step and a counting time of 2-3 seconds/step. Each scan took 5-10 minutes. Scans were taken repeatedly during the growth of the Pt(111) and Pt(200) diffraction peaks, for 1080 minutes (18 hours). 


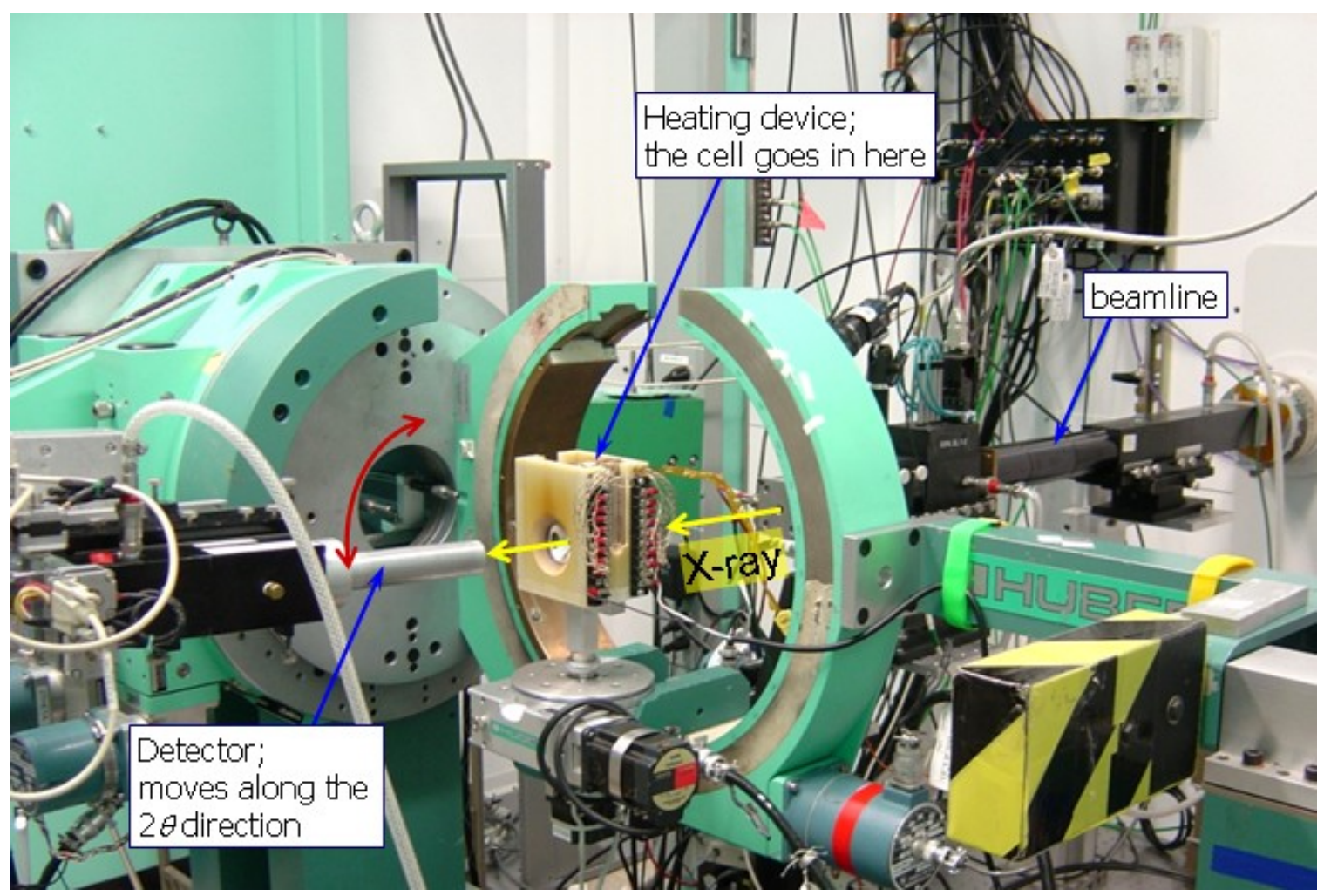

Figure 2-6. In situ XRD setup at beamline 7-2 at SSRL.

\subsection{Transmission Electron Microscopy (TEM)}

TEM and TEM-based techniques were the primary characterisation techniques used in this project. These techniques include TEM and high-resolution TEM (HRTEM) imaging, selected area electron diffraction (SAED), and energy-dispersive X-ray spectroscopy (EDS). Each technique is useful for providing certain information about the sample, when combined the techniques become a powerful tool for determining the size, shape, composition, and crystal structure of the materials studied. Detailed information of the basic principles of the TEM and TEM-based techniques, and the applications of these techniques can be found in Williams and Carter.[9]

\section{Basics of TEM}

TEM is an imaging technique that utilises a high-energy beam of electrons to visualise specimens. Due to the charged nature of electrons, magnetic fields can be used to manipulate and focus the electron beam to produce structural images and diffraction 
patterns of materials. Figure 2-7 shows a schematic of a transmission electron microscope column.

A lanthanum hexaboride $\left(\mathrm{LaB}_{6}\right)$ single crystal is used as the electron source for the electron microscope in our laboratory. The electrons are accelerated by an applied potential difference of $200 \mathrm{keV}$. Due to relativistic effects on the electrons caused by the acceleration by the large potential difference, the precise wavelength of the electrons, $\lambda$, is given by

$$
\left.\lambda=\frac{h\left(1-\beta^{2}\right)^{1 / 4}}{\left(2 m_{e} e_{0} E\right)^{1 / 2}} \quad \text { (Eq. 2.4 }\right)^{[9]}
$$

where $h$ is Plank's constant, $m_{e}$ is the mass of an electron, $e_{0}$ is the electronic charge, $E$ is the accelerating voltage and $\beta=v / c$ where $v$ is the velocity of the electron and $c$ the speed of light in a vacuum.

These fast moving electrons have wavelengths $(\lambda=0.0251 \AA$ at $200 \mathrm{keV})$ much smaller than the inter-atomic distances in a crystal lattice and are thus diffracted by the atoms when they interact with a crystal.

The electrons generated by the electron source are first focused by the condenser lens to form an approximately parallel beam and strike the specimen. A ray diagram between the specimen and the screen is shown in Figure 2-8.[9] Some of the electrons are diffracted by the sample imparting structural information to the beam. The electron beam wavefront can then be focused to a point which lies on a plane. This produces an electron diffraction pattern. This plane is the back focal plane of the objective lens and is normal to the straight-through beam. When the microscope is operated in the diffraction mode, this diffraction pattern is projected down the microscope. When operated in the imaging mode, the diffracted and undiffracted beams are recombined by the objective lens to produce an image of the sample. The is further magnified by the magnification system consisting of intermediate and projector lenses. Thus, by adjusting the lens configuration the image or the diffraction pattern can be projected down the microscope. Both the projected image and diffraction pattern can be recorded on a fluorescent screen or photographic plate, or captured by a digital camera. 


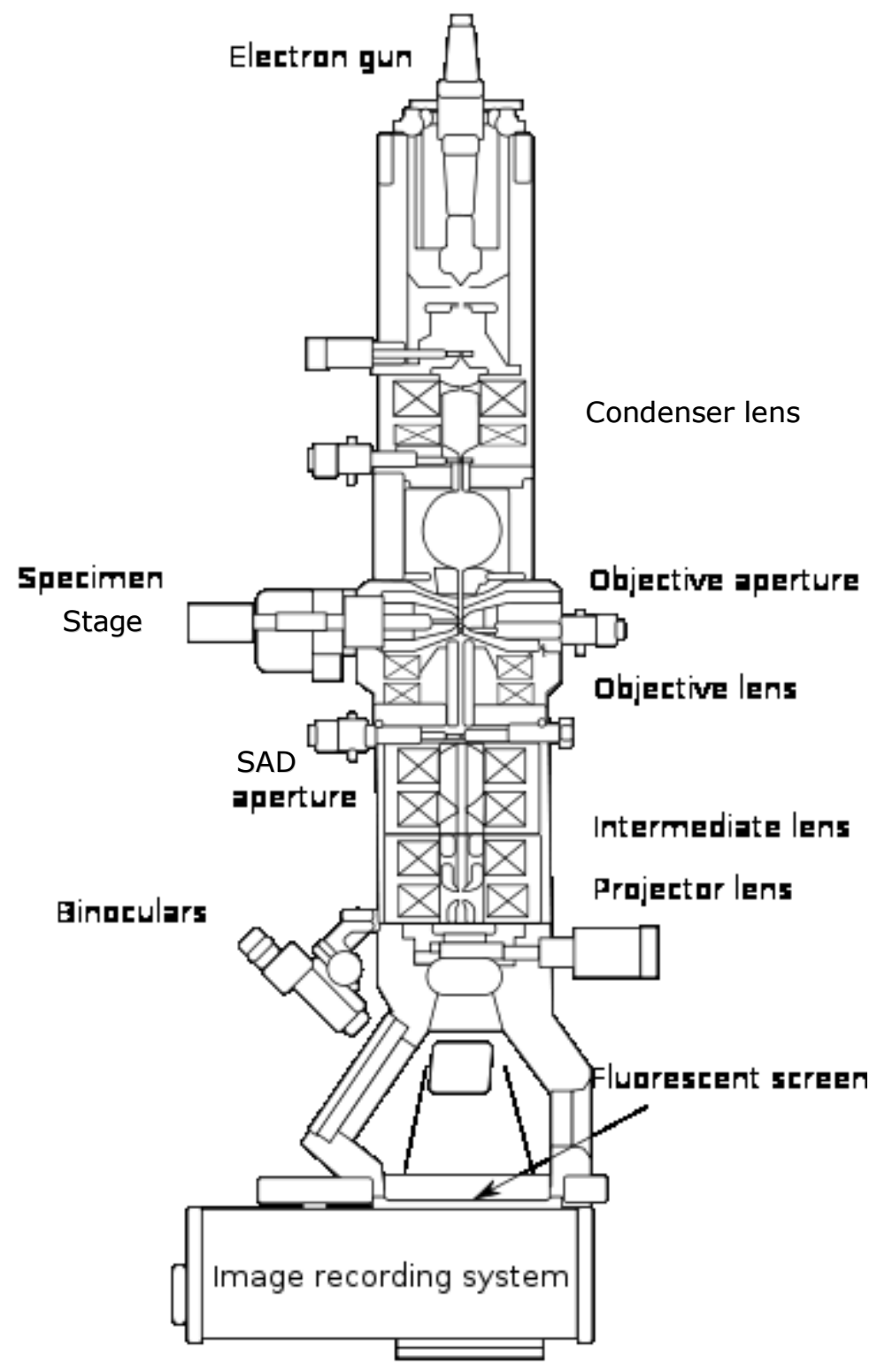

Figure 2-7. Schematic showing the layout of the optical components in a transmission electron microscope.

A transmission electron microscope has the same functional components as a light microscope. The electron gun generates electrons that behave the same manner as light does in a vacuum. (The entire microscope column is operated under vacuum.) The lenses are electromagnetic lenses, which have the same effect on electrons as glass lenses on visible light. The specimen is inserted at the specimen stage, where a goniometer is used to tilt the specimen. The TEM image generated can be viewed at the fluorescent screen or be captured by the imaging recording system. 


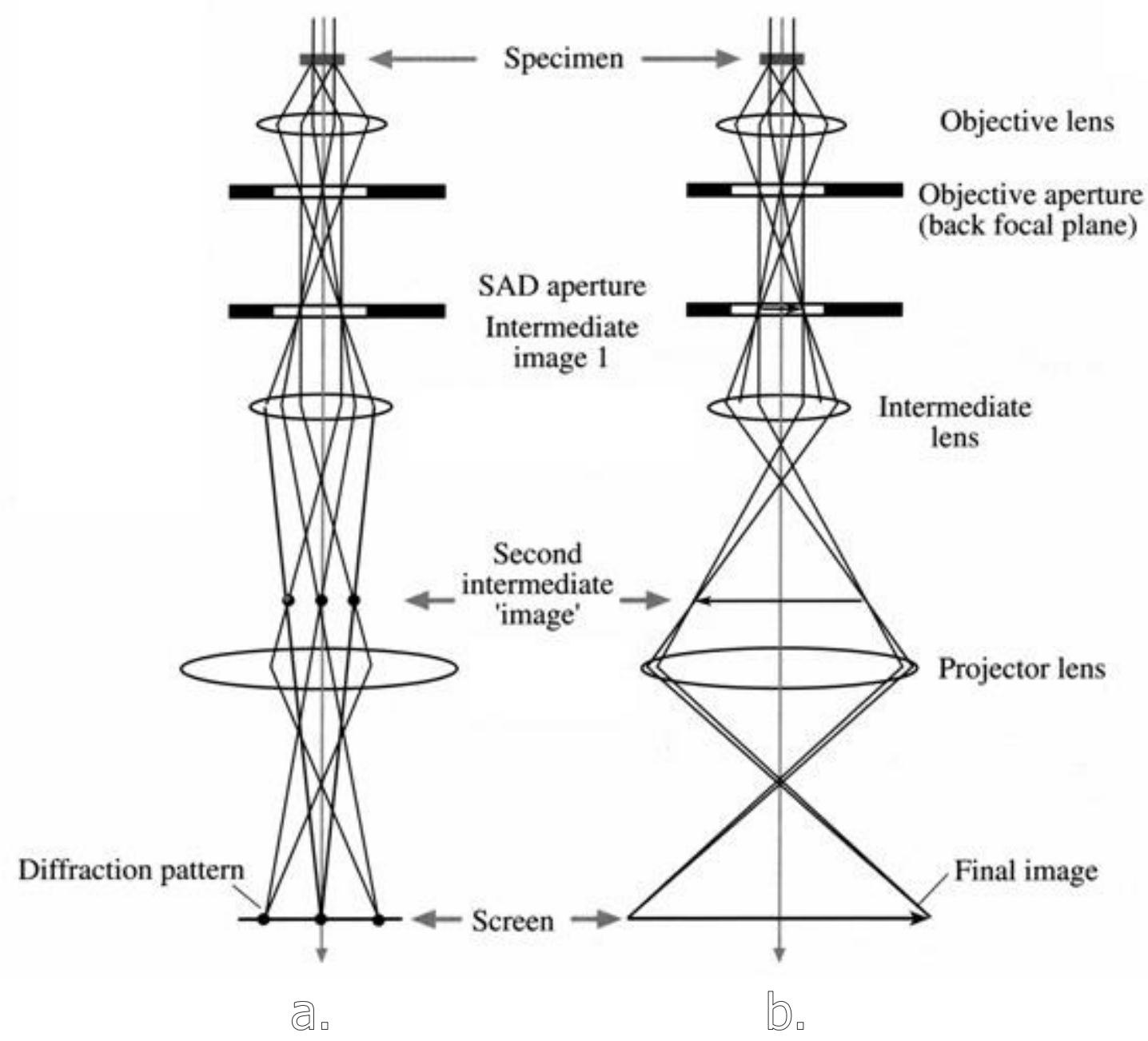

Figure 2-8. Ray diagram of a transmission electron microscope in the a. diffraction and b. imaging modes.

\subsubsection{High-Resolution TEM (HRTEM) Imaging}

HRTEM enables direct probing of structural information of crystalline nanostructures. Highly detailed images at atomic resolution may be obtained, but one has to be aware that a TEM image is formed by recombination of the diffracted and undiffracted electron beams. It is thus important to consider the constraints that the electron microscope itself imposes on the information in the image.

The actual lens in an electron microscope is the magnetic field within the vacuum between the pole-pieces of the objective lens, and the geometry of this field is difficult to control. One consequence of this is that beams which are diffracted through different angles are brought into focus at slightly different planes. This is known as spherical aberration. To some extent this effect can be countered for by defocusing the beam. When the focus is adjusted by an 
amount $\Delta \mathrm{F}$, i.e. defocus, in the microscope the focal length of the objective lens is altered. The spherical aberration coefficient of the objective lens, $\mathrm{C}_{\mathrm{s}}$ and $\Delta \mathrm{F}$ are related by the phase contrast transfer function, $\mathrm{T}(\mathrm{u})$, given by

$$
\mathrm{T}(\mathrm{u})=2 \mathrm{~A}(\mathrm{u}) \sin [\chi(\mathrm{u})] \quad(\text { Eq. 2.5 })^{[9]}
$$

where $u$ is a reciprocal lattice vector, $A(u)$ is the aperture function, and $\chi(u)$ is the phase-distortion function, given by

$$
\left.\chi(\mathrm{u})=\pi \Delta \mathrm{F} \lambda \mathrm{u}^{2}+\frac{1}{2} \pi \mathrm{C}_{\mathrm{s}} \lambda^{3} \mathrm{u}^{4} \quad \text { (Eq. 2.6) }\right)^{[9]}
$$

$\mathrm{T}(\mathrm{u})$ describes the way in which phase shifts are introduced into the diffracted beams and this in turn converts the phase difference into an amplitude difference in the image intensity. $T(\mathrm{u})$ is at a maximum when $\chi(\mathrm{u})$ is a multiple of $\pm 1 / 2 \pi$, and at zero when $\chi(\mathrm{u})$ is a multiple of $\pm \pi$. Hence the appearance of an HRTEM image is largely determined by the phase shift of electron wave. At negative $\mathrm{T}(\mathrm{u})$, the phase contrast is positive and atoms appear dark against a bright background. Conversely, when T(u) is positive, phase contrast is negative and atoms appear bright against a dark background. There is no detail in the image when $\mathrm{T}(\mathrm{u})$ is at zero.

As $\Delta \mathrm{F}$ and $\mathrm{C}_{\mathrm{s}}$ are opposite there exists a value of defocus where $\Delta \mathrm{F}$ and $\mathrm{C}_{\mathrm{s}}$ effectively cancel out and this is known as the Scherzer defocus, $\Delta \mathrm{F}_{\text {Sch, }}$ which occurs at

$$
\left.\Delta \mathrm{F}_{\mathrm{Sch}}=-1.2\left(\mathrm{C}_{\mathrm{s}} \lambda\right)^{\frac{1}{2}} \quad \text { (Eq. 2.7 }\right)^{[9]}
$$

It is derived that at this defocus, all the beams have nearly constant phase and hence the same relative intensities. This condition designates the interpretable resolution limit of the transmission electron microscope, and typically lies within the range of 1.6-2.0 ̊.

Two other important factors that affect the resolution of the microscope are the chromatic aberration and beam divergence. Chromatic aberration originates from the fact that the focal length of the magnetic lens is dependent on the energy of the electrons. The electron beam is not perfectly monochromatic having a spread of energies and consequently wavelengths. The inherent thermal energy spread of electrons emitted from the electron 
source and fluctuations in the accelerating voltage and the objective lens current would also contribute to the occurrence of chromatic aberration.

Beam divergence results from the filament not being a point source and consequently the incident beam is never exactly parallel. Both chromatic aberration and beam divergence reduce the performance of the microscope by introducing an uncertainty in the phase introduced by the objective lens.

In this project, a JEOL 2010 operated at $200 \mathrm{keV}$ was used for TEM and HRTEM imaging. The instrumental resolution limit is about 1.94 Å.[10]

\section{Image interpretation}

As the observed image contrast is severely dependent on instrumental factors such as the spherical aberration and the objective lens defocus, characterisation has to be done with care.

Characterisation was performed by comparing the HRTEM image with that calculated from a structure model and assessing the degree of matching. The Gatan Digital Micrograph software suite was used to capture digital micrographs and to perform fast Fourier transform functions on some images. Structure models and simulated diffraction patterns were generated using CARINE 3.1.

The procedure is illustrated in Figure 2-9, using a HRTEM image of a ruthenium nanoparticle. A fast Fourier transform (FFT) function is performed on the HRTEM image to produce a power spectrum of the image. An FFT transforms a function from a spatial domain to a frequency domain. In the context of an image, lattice fringes are repeating features and would show up as spots corresponding to the frequency of repetition. The angular relationship between the fringes is maintained for the spots. The power spectrum could be compared to a simulated diffraction pattern from a model of the specimen under study. The structure of a sample is considered properly characterised when matched simulations could be obtained for a number of nanoparticles and the results agreed with those from electron diffraction and energy dispersive spectroscopy compositional analysis. This was done routinely in many investigations in order to properly characterise a sample. 

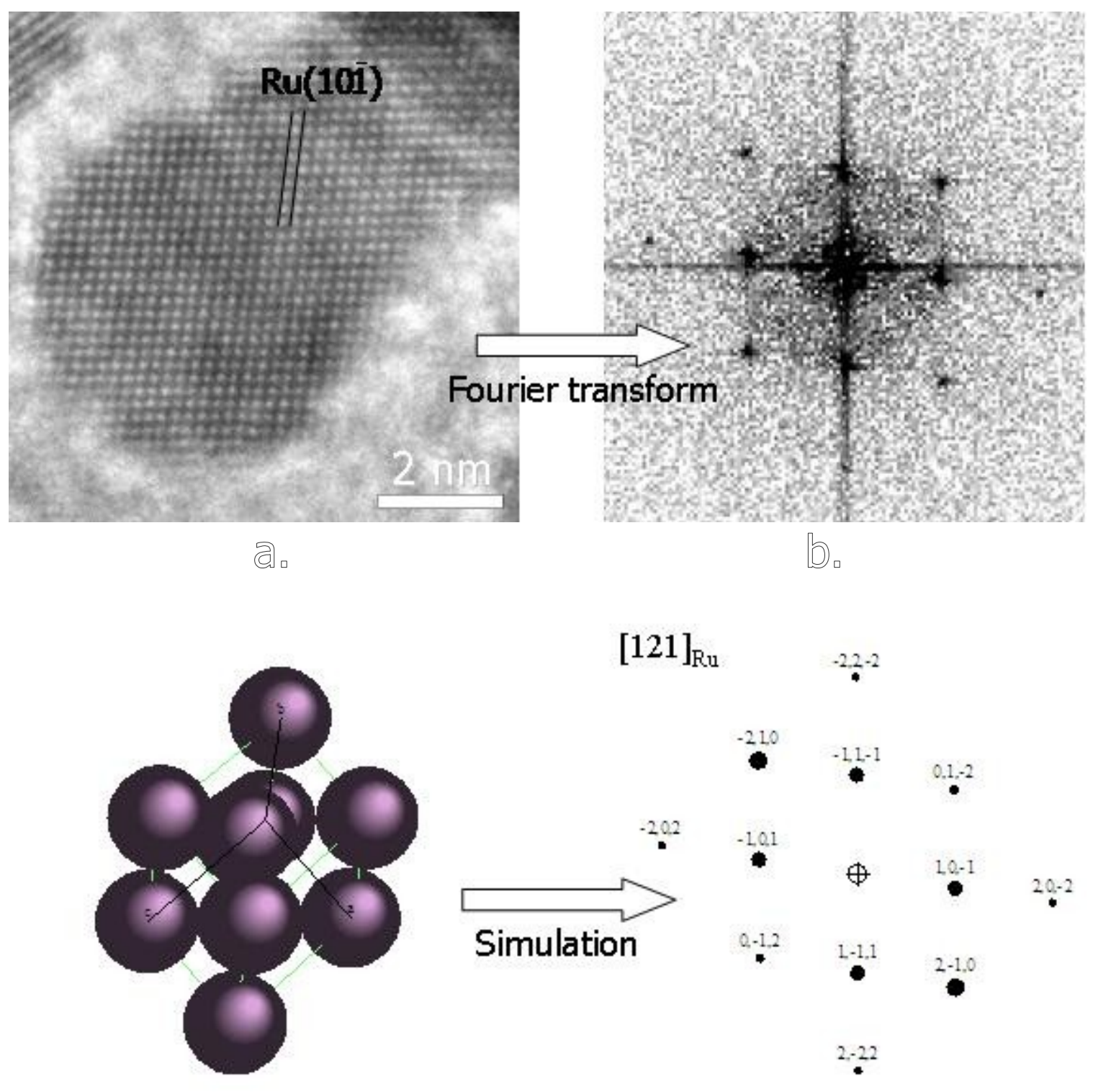

Figure 2-9. Interpretation of HRTEM image.

a. HRTEM image showing part of a ruthenium nanocrystal. b. Power spectrum of image a.

c. Structure model of ruthenium viewed down a [121] zone axis. d. Simulated diffraction pattern along the [121] direction.

\subsection{Selected Area Electron Diffraction (SAED)}

Electron diffraction analysis was used considerably to gain structural information of the sample. Often, both electron microscopy images (information in real space) and diffraction patterns (information in reciprocal space) are obtained for the same region. Electron diffraction can be performed on a single nanoparticle or an area consisting of multiple 
nanoparticles, by inserting the relevant selected area aperture(s) - hence selected area electron diffraction (SAED).

\section{Data interpretation}

The electron diffraction pattern can be used to differentiate between amorphous and crystalline particles; amorphous material would give rise to a broad, diffuse ring pattern, in contrast to a crystalline material that would produce discrete spots or rings. Indexing the diffraction pattern of a crystalline material then yields the crystal structures present in the sample, and sometimes the orientation of the nanocrystals.

Electron diffraction patterns were recorded on Kodak S0-163 electron microscopy film plates. The distance between two symmetry-related diffraction spots, or the diameter or radius of a diffraction ring can be measured on the developed negative. As illustrated in Figure $2-10,{ }^{[9]}$ the diffraction radius, $R$ is related to the Bragg angle, $\theta$ (half the angle between the direct beam and the diffracted beam) by

$\tan 2 \theta=\frac{R}{L} \quad$ (Eq. 2.8) ${ }^{[9]}$

where $L$ is the camera length - distance between the specimen and the image plane.

In the electron microscope, the camera length $L$ is a calculated value rather than a physical distance. The effective $L$ value can be increased by increasing the magnification of the lenses.

Since $\theta$ from electron diffraction is usually very small, $\tan 2 \theta \approx ! \theta \approx \sin \theta$ is valid. Substituting this and Eq. 2.8 into the Bragg equation (Eq. 2.1) thus gives

$$
\frac{R}{L}=\frac{\lambda}{d} \quad \text { (Eq. 2.9) }
$$

This can then be re-written as

$$
d=\frac{2 \lambda L}{D} \quad \text { (Eq. 2.10) }
$$

where $D$ is the diameter of the diffraction ring. 
Thus the interplanar spacing can be calculated. The term $2 \lambda L$, called the camera constant, was determined by calibration from the diffraction pattern of platinum nanocrystals.

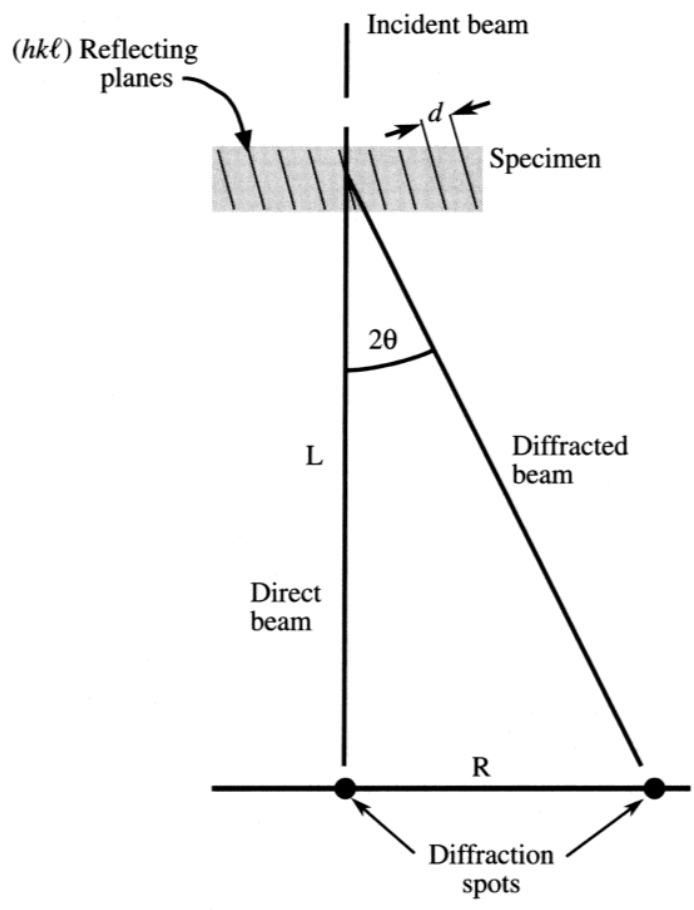

Figure 2-10. Schematic illustrates the relationship between camera length, Bragg angle, and the radius of a diffraction ring in SAED.

\subsection{Energy Dispersive X-Ray Spectroscopy (EDS)}

EDS is a supplementary technique to TEM. It utilises X-rays that are emitted from the atoms in the sample excited by the electron beam to characterise the elemental composition of the analysed volume.

In this project, EDS analysis was mainly conducted for ruthenium nanoparticles. EDS spectra were acquired using the JEOL Analysis Manager software suite.

A specimen being bombarded by the electron beam in the electron microscope emits X-rays as a result of inelastic scattering of electrons by the specimen. An inelastically scattered electron is lower in energy when compared to the incident electron. 
Electrons generated at the microscope contain sufficient energy to ionise atoms by exciting and releasing an electron from one of the inner shells (lower energy shells) before moving on with reduced energy. An electron from an outer shell (higher energy shell) would then fall into the inner shell to fill in the vacancy thus stabilising the atom. As the electron drops from a higher energy to a lower energy shell, energy can be lost either as the emission of a an Auger electron or an X-ray photon. The energy of the Auger electron or the X-ray photon is characteristic of an element and can be used as a "fingerprint" to indicate the presence of a specific element within a crystal.

\subsection{Magnetometry}

Magnetic measurements were performed using a Quantum Design magnetic properties measurement system (MPMS) at Industrial Research Limited. It comprises a superconducting quantum interference device (SQUID) detector and a variable-field superconducting magnet equipped with a temperature controller within its bore.

The sample was first pressed into a pellet, weighed, and secured in a gelatine capsule and sealed. The gelatine capsule was fixed in a plastic straw, which was fastened to the sample rod, and inserted through an airlock into the helium-filled bore of the MPMS. A mechanical drive attached to the sample rod was used to move the sample vertically within the bore until it was centred within the SQUID detector coils.

Magnetic loop (or hysteresis loop) measurements were conducted. The magnetic loop measures the induced magnetisation of the sample as a function of applied field strength. The measurement result could give an indication of the magnetic behaviour of the sample, such as ferromagnetic or superparamagnetic behaviour. In a typical measurement, a magnetic field, $H$, was applied to the sample in a progressive fashion, while the magnetisation of the sample, $M$, was measured against the applied field. The field was varied between $+60 \mathrm{kOe}$ and $-60 \mathrm{kOe}(1 \mathrm{kOe}=0.1 \mathrm{~T})$. The interval between data points was $10 \mathrm{kOe}$ at high field $(H>+10 \mathrm{kOe}$ and $H<-10 \mathrm{kOe})$ and $1 \mathrm{kOe}$ at low field ($10 \mathrm{kOe}<H<+10 \mathrm{kOe})$. After being increased from 0 to $+60 \mathrm{kOe}$, the applied field was decreased progressively to zero, and then applied progressively in the opposite direction to $-60 \mathrm{kOe}$, at which the applied field was swept back to $+60 \mathrm{kOe}$. 


\subsection{References}

[1] M. Ladd and R. Palmer, Structure Determination by X-Ray Crystallography. (Kluwer Academic, New York, 2003).

[2] C. Giacovazzo (ed.) Fundamentals of Crystallography. (Oxford University Press, Oxford, U.K., 2002).

[3] http://www.alphamaterials.com/SiC_powder.htm (Alpha Materials Inc., last accessed on Oct 2009).

[4] H. Borchert, E. V. Shevchenko, A. Robert, I. Mekis, A. Kornowski, G. Grubel and H. Weller, Determination of nanocrystals sizes: a comparison of TEM, SAXS, and XRD studies of highly monodisperse $\mathrm{CoPt}_{3}$ particles. Langmuir 21, 1931-1936 (2005).

[5] H. Natter, M. Schmelzer, M. S. Loffler, C. E. Krill, A. Fitch and R. Hempelmann, Graingrowth kinetics of nanocrystalline iron studied in situ by synchrotron real-time X-ray diffraction. Journal of Physical Chemistry B 104, 2467-2476 (2000).

[6] http://www.synchrotron.org.au (Australian Synchrotron, last accessed on Jan 2008).

[7] http://www-ssrl.slac.stanford.edu (Stanford Synchrotron Radiation Lightsource, SLAC National Accelerator Laboratory, last accessed on Nov 2009).

[8] H. Wiedemann, Synchrotron Radiation. (Springer, Berlin, New York, 2003).

[9] D. B. Williams and C. B. Carter, Transmission Electron Microscopy. A Textbook for Materials Science, Vol. I-IV. (Plenum Press, New York, 1996).

[10] http://www.jeolusa.com/PRODUCTS/ElectronOptics/ TransmissionElectronMicroscopesTEM/200kV/JEM2100LaB6/tabid/207/Default.aspx (JEOL USA, Inc., last accessed on Dec 2009). 


\section{Chapter 3}

\section{Synthesis and Characterisation of Iron Nanoparticles}

\section{Chapter outline}

\subsection{Introduction}

\subsection{Fischer-Porter Bottle Synthesis}

3.2.1. Precursor Concentration

3.2.2. Temperature

3.2.3. Stabilising Agent

3.2.4. Other Variables

3.2.5. Formation of Iron Oxide Nanoparticles

3.3. Bench-Top Synthesis

3.4. Discussion

3.4.1. Nanoparticle Composition

3.4.2. Nanoparticle Size

3.4.3. Stability of Nanoparticles

3.4.4. Magnetic Properties

3.5. Chapter Summary

3.6. Experimental

3.7. References

\subsection{Introduction}

The research work on the synthesis and characterisation of iron-based nanoparticles is presented in two chapters. This chapter describes the synthesis of iron nanoparticles at temperatures $130-180^{\circ} \mathrm{C}$, and discusses how the experimental conditions affect the nanoparticle composition, morphology and size. 
The initial aim of the research was to synthesise iron nanoparticles of larger than $10 \mathrm{~nm}$ in size, which would oxidise on the surface leading to core/shell structures. The extreme reactivity of iron (particularly with water and oxygen) has made any fundamental and applicational studies of iron nanoparticles very difficult and inconvenient. ${ }^{[1]}$ An iron nanoparticle of $8 \mathrm{~nm}$ or smaller would oxidise completely upon exposure to air. For a larger nanoparticle, an oxide layer of 3-4 $\mathrm{nm}$ forms on the nanoparticle surface almost instantly when the nanoparticle is exposed to air.[2,3] The oxide layer is able to act as a passivation shell that prevents further oxidation, resulting in a stable core/shell structure. Besides, the oxide shell can be readily functionalised for the desired applications (for example bioseparation and targeted drug delivery), while the iron core remains the major source of high magnetisation. ${ }^{[4-6]}$

This research also aimed to explore a facile synthetic route at temperatures below $200{ }^{\circ} \mathrm{C}$. The literature on iron nanoparticle synthesis was recently reviewed by Huber ${ }^{[1]}$ and Tavakoli et al.[7] The most commonly adopted solution-phase synthesis of iron nanoparticles is thermal decomposition of iron pentacarbonyl $\left(\mathrm{Fe}(\mathrm{CO})_{5}\right)$ at $150-300{ }^{\circ} \mathrm{C}$, in the presence of stabilising molecules. The frequent use of $\mathrm{Fe}(\mathrm{CO})_{5}$ is mainly because it can be readily decomposed and purification can be fairly easy as there is no by-product from the reaction, apart from gaseous carbon monoxide. However, the toxicity and volatility of $\mathrm{Fe}(\mathrm{CO})_{5}$ have prevented further development of this synthesis. Decompositions of other iron precursors have been investigated, such as iron oleate by Hyeon and co-workers,[8,9] and an iron amido complex by Chaudret and co-workers.[10,11] Apart from thermal decomposition, other preparations of iron nanoparticles in solution include decomposition of $\mathrm{Fe}(\mathrm{CO})_{5}$ using sonochemical methods, ${ }^{[12]}$ and the reduction of iron salts such as $\mathrm{FeCl}_{3}$ in reverse micelles.[13,14] Nevertheless, literature reports of iron nanoparticles of larger than $8 \mathrm{~nm}$ are limited. Most of the iron nanoparticles reported were handled in an oxygen-free atmosphere, from preparation to characterisation, due to the small size of the nanoparticles obtained. It is therefore highly desirable to develop a facile synthesis that produces iron-based nanoparticles that can be almost readily put into applications. 


\subsection{Fischer-Porter Bottle Synthesis}

Synthesis of iron nanoparticles using Fischer-Porter bottle was first reported by Chaudret and co-workers, which involved the decomposition of an iron amido complex $\mathrm{Fe}\left[\mathrm{N}\left(\mathrm{SiMe}_{3}\right)_{2}\right]_{2}\left(\mathrm{Me}=\mathrm{CH}_{3}\right)$ under 4 bar $\mathrm{H}_{2}(1 \mathrm{bar}=100 \mathrm{kPa}) \cdot{ }^{[10]}$ Iron nanoparticles of up to $7 \mathrm{~nm}$ were prepared, but had to be handled in an oxygen-free atmosphere to prevent complete oxidation.

In a recent study of Fischer-Porter bottle synthesis, Ren from our group has shown promising results by using $\mathrm{Fe}\left(\mathrm{C}_{5} \mathrm{H}_{5}\right)\left(\mathrm{C}_{6} \mathrm{H}_{7}\right)$ as the precursor.[15] Among the many precursors and surfactant/solvent systems attempted, core/shell nanoparticles of larger than $10 \mathrm{~nm}$ were reported for samples prepared from decomposition of $\mathrm{Fe}\left(\mathrm{C}_{5} \mathrm{H}_{5}\right)\left(\mathrm{C}_{6} \mathrm{H}_{7}\right)$, in the presence of oleylamine as the stabilising agent. However, shape and size control, and the required conditions for nanoparticle growth were not clear. This research work has adopted the same reaction system and extended the study in order to gain an understanding and possibly control of the synthesis. The experimental conditions, along with the morphology and size of nanoparticles obtained, are listed in Table 3-1. Further experiments undertaken at $120^{\circ} \mathrm{C}$ and below are described in Chapter 4 . 
Table 3-1. Summary table of Fischer-Porter bottle synthesis.

\begin{tabular}{|c|c|c|c|c|c|c|c|}
\hline Expt & $\begin{array}{c}\text { Conc. }^{A} \\
\text { (M) }\end{array}$ & OLAB/Fe & $\begin{array}{l}\text { Temp. } \\
\left({ }^{\circ} \mathrm{C}\right)\end{array}$ & Time $^{\mathrm{C}}$ & $\begin{array}{l}\mathrm{H}_{2} \text { press. } \\
\left(10^{2} \mathrm{kPa}\right)\end{array}$ & Nanoparticles & $\begin{array}{c}\text { Avg. size } \\
\text { (nm) }\end{array}$ \\
\hline $\mathrm{Fe} \_\mathrm{A}$ & 0.05 & 3 & 130 & $2 d$ & 1 & Iron oxide & $7 \pm 2$ \\
\hline $\mathrm{Fe}_{-} \mathrm{B}$ & 0.25 & 3 & 130 & $2 \mathrm{~d}$ & 1 & Core/shell, spherical & $14 \pm 2$ \\
\hline $\mathrm{Fe}_{-} \mathrm{C}$ & 0.25 & 3 & 130 & $2 d$ & 3 & Core/shell, spherical & $14 \pm 2$ \\
\hline Fe_D & 1.0 & 3 & 130 & $2 d$ & 1 & Polydisperse & $3-30$ \\
\hline $\mathrm{Fe}_{-} \mathrm{E}$ & 0.25 & 3 & 140 & $2 d$ & 1 & Core/shell, spherical & $14 \pm 2$ \\
\hline $\mathrm{Fe}_{-} \mathrm{F}$ & 0.25 & 3 & 150 & $2 \mathrm{~h}$ & 1 & Iron oxide & $6 \pm 2$ \\
\hline $\mathrm{Fe}_{-} \mathrm{G}$ & 0.25 & 3 & 160 & $2 \mathrm{~h}$ & 1 & Iron oxide & $6 \pm 2$ \\
\hline $\mathrm{Fe}_{-} \mathrm{H}$ & 0.25 & 3 & 160 & $2 \mathrm{~h}$ & 2 & Iron oxide & $6 \pm 2$ \\
\hline $\mathrm{Fe}_{-} \mathrm{I}$ & 0.25 & 0.2 & 130 & $2 d$ & 1 & Iron oxide & $6 \pm 3$ \\
\hline $\mathrm{Fe}_{-} \mathrm{J}$ & 0.25 & 1 & 130 & $2 d$ & 1 & Core/shell, spherical & $14 \pm 2$ \\
\hline $\mathrm{Fe}_{-} \mathrm{K}$ & 0.25 & 6 & 130 & $2 \mathrm{~d}$ & 1 & Core/shell, spherical & $16 \pm 5$ \\
\hline
\end{tabular}

A Mesitylene was used as the solvent.

${ }^{B}$ OLA = oleylamine

${ }^{\mathrm{C}} \mathrm{d}=$ day, $\mathrm{h}=$ hour

\subsubsection{Precursor Concentration}

Precursor concentration was observed to be one of the main factors contributing to the formation of iron nanoparticles of larger than $10 \mathrm{~nm}$. Precursor concentrations of 0.05, 0.25, and $1.0 \mathrm{M}$ were investigated at $130{ }^{\circ} \mathrm{C}$. Oleylamine was used as the stabiliser, with a mole ratio of oleylamine $:$ iron $=3: 1$.

\section{Expt Fe_A. Reaction of $0.05 \mathrm{M}$ iron precursor in mesitylene (low concentration)}

At the end of the experiment, the reaction solution was dark brown to black in colour. The black precipitate was collected via purification by magnetic separation. Figure 3-1a shows a TEM image of the nanoparticles obtained, which were spherical or near-spherical in shape, averaging $7 \mathrm{~nm}$, and had aggregated. The SAED pattern in Figure 3-1b can be indexed to iron oxide of the spinel phases - magnetite $\left(\mathrm{Fe}_{3} \mathrm{O}_{4}\right)$, or maghemite $\left(\gamma\right.$ - $\left.\mathrm{Fe}_{2} \mathrm{O}_{3}\right)$, or both - as 
shown in Table 3-2. No crystalline iron was identified from the diffraction pattern. The calculated $d$-spacings were closer to the bulk values of $\mathrm{Fe}_{3} \mathrm{O}_{4}(+0.04 \pm 0.01 \AA)$ than those of $\gamma-\mathrm{Fe}_{2} \mathrm{O}_{3}(+0.05 \pm 0.01 \AA$ ) . For this reason and the colour observed during purification, it is believed that the sample consisted of mainly the $\mathrm{Fe}_{3} \mathrm{O}_{4}$ phase.
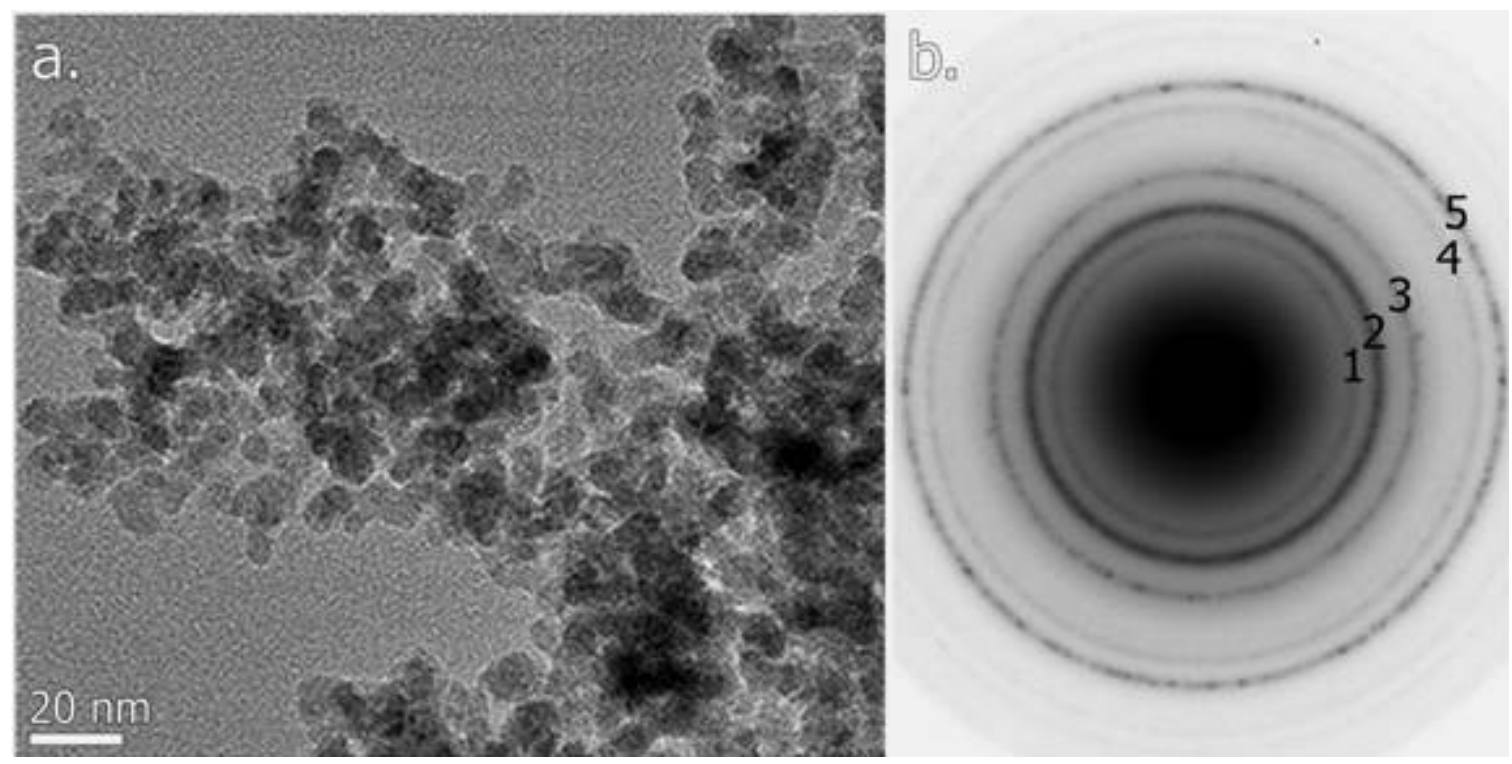

Figure 3-1. TEM and SAED results for low-concentration reaction at $130{ }^{\circ} \mathrm{C}\left(\mathrm{Fe} \_\mathrm{A}\right)$.

a. TEM image showing aggregates of nanoparticles of $7 \mathrm{~nm}$ in average diameter. $\mathbf{b}$. The corresponding SAED pattern was indexed to iron oxide of the spinel phases (See Table 3-2).

Table 3-2. Index for SAED pattern in Figure 3-1b.

\begin{tabular}{|c|c|c|c|c|c|c|}
\hline Index & $\begin{array}{l}\text { Calc. } d \\
(\AA \AA)\end{array}$ & $\begin{array}{c}\text { Matched } d \text { of } \mathrm{Fe}_{3} \mathrm{O}_{4} \\
\text { (Å) }\end{array}$ & $\begin{array}{c}\text { Intensity } \\
\text { (\%) }\end{array}$ & $\begin{array}{c}\text { Matched } d \text { of } \gamma-\mathrm{Fe}_{2} \mathrm{O}_{3} \\
(\AA \AA)\end{array}$ & $\begin{array}{c}\text { Intensity } \\
\text { (\%) }\end{array}$ & $\begin{array}{c}\left(\mathrm{h} \mathrm{kl)} \mathrm{of} \mathrm{Fe}_{3} \mathrm{O}_{4} \text { or }\right. \\
\gamma-\mathrm{Fe}_{2} \mathrm{O}_{3}\end{array}$ \\
\hline 1 & 2.99 & 2.9678 & 30 & 2.9530 & 35 & $\left(\begin{array}{lll}2 & 2 & 0\end{array}\right)$ \\
\hline 2 & 2.58 & 2.5309 & 100 & 2.5177 & 100 & $\left(\begin{array}{lll}3 & 1 & 1\end{array}\right)$ \\
\hline 3 & 2.13 & 2.0985 & 20 & 2.0886 & 16 & $\left(\begin{array}{lll}4 & 0 & 0\end{array}\right)$ \\
\hline 4 & 1.66 & 1.6154 & 28 & 1.6073 & 24 & $\left(\begin{array}{lll}5 & 1 & 1\end{array}\right)$ \\
\hline 5 & 1.52 & 1.4839 & 36 & 1.4758 & 34 & $\left(\begin{array}{lll}4 & 4 & 0\end{array}\right)$ \\
\hline
\end{tabular}




\section{Expt Fe_B. Reaction of $0.25 \mathrm{M}$ iron precursor in mesitylene (middle concentration)}

When the same reaction was carried out with 5 times the precursor concentration, a strongly magnetic, black precipitate was collected. During purification, the precipitate in solution was observed to be attracted to the magnet almost instantly. In Expt Fe_A, it took about 5 minutes for all of the precipitate to be attracted to the magnet.

As shown in Figure 3-2a, near-monodisperse, spherical and near-spherical nanoparticles were obtained. The nanoparticle size distribution is shown in Figure 3-2b. The size monodispersity was also indicated by the hexagonal monolayer assembly displayed by the nanoparticles. The nanoparticles comprised a core/shell structure, typically with a core averaging at $9 \pm 1 \mathrm{~nm}$ and a shell thickness of $2.6 \pm 0.6 \mathrm{~nm}$. The different contrast between the core and the shell indicated a heterogeneous structure within the individual nanoparticles. This was also revealed in the SAED pattern in Figure 3-2c. Additional diffraction rings to those of iron oxide were observed, and could be indexed to $\alpha$-Fe, as shown in Table 3-3.

Figure 3-3 shows the powder XRD pattern of the sample, in which peaks were indexed to the respective reflections of $\alpha$-Fe and spinel iron oxide. The diffraction rings in the SAED pattern and the peaks in the XRD pattern that correspond to $\alpha$-Fe were of higher intensity compared to those of iron oxide. The XRD peaks of iron oxide were also noticeably broader. These observations indicated that the $\alpha$-Fe crystallites present in the sample were larger in amount and in size compared to those of the iron oxide. From the diffractogram, the average crystallite size estimated from the fwhm of $\mathrm{Fe}(110)$ and $\mathrm{Fe}(211)$ peaks was $8.0 \pm 0.6 \mathrm{~nm}$, which agreed well with the average size of the core of the nanoparticles as observed in the TEM. 


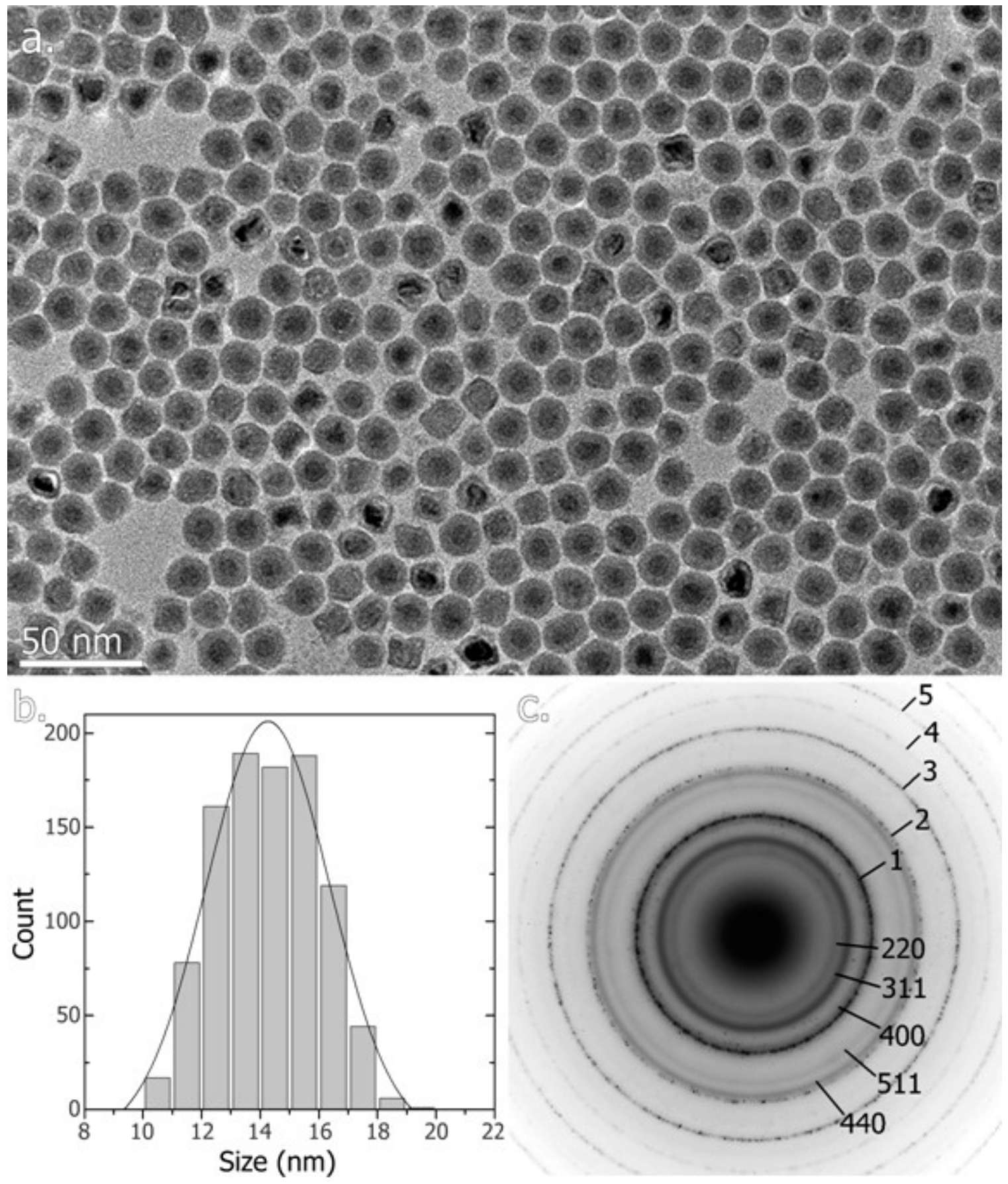

Figure 3-2. TEM and SAED results for middle-concentration reaction at $130^{\circ} \mathrm{C}\left(\mathrm{Fe} \_\mathrm{B}\right)$.

a. TEM image of near-monodisperse core/shell nanoparticles, most of which arranged into monolayer of hexagonal assembly. $\mathbf{b}$. Size distribution histogram of 985 nanoparticles selected from random areas of different TEM grids; mean size $=14.3 \mathrm{~nm}$, standard deviation $=1.7 \mathrm{~nm}$ (12\%). c. SAED pattern showing diffraction rings of $\alpha$-Fe (see Table 3-3) and spinel iron oxide (labelled). 
Table 3-3. Index for the non-iron oxide diffraction rings in Figure 3-2c.

\begin{tabular}{|c|c|c|c|c|}
\hline Index & $\begin{array}{c}\text { Calc. } \boldsymbol{d} \\
(\AA)\end{array}$ & $\begin{array}{c}\text { Matched } \boldsymbol{d} \text { of } \boldsymbol{\alpha} \text {-Fe } \\
(\AA)\end{array}$ & $\begin{array}{c}\text { Intensity } \\
(\mathbf{\%})\end{array}$ & $\mathbf{h} \mathbf{~ k ~}$ \\
\hline 1 & 2.05 & 2.0260 & 100 & 110 \\
\hline 2 & 1.45 & 1.4326 & 12 & 200 \\
\hline 3 & 1.18 & 1.1697 & 20 & 211 \\
\hline 4 & 1.02 & 1.0130 & 5 & 220 \\
\hline 5 & 0.91 & 0.9060 & 8 & 310 \\
\hline
\end{tabular}

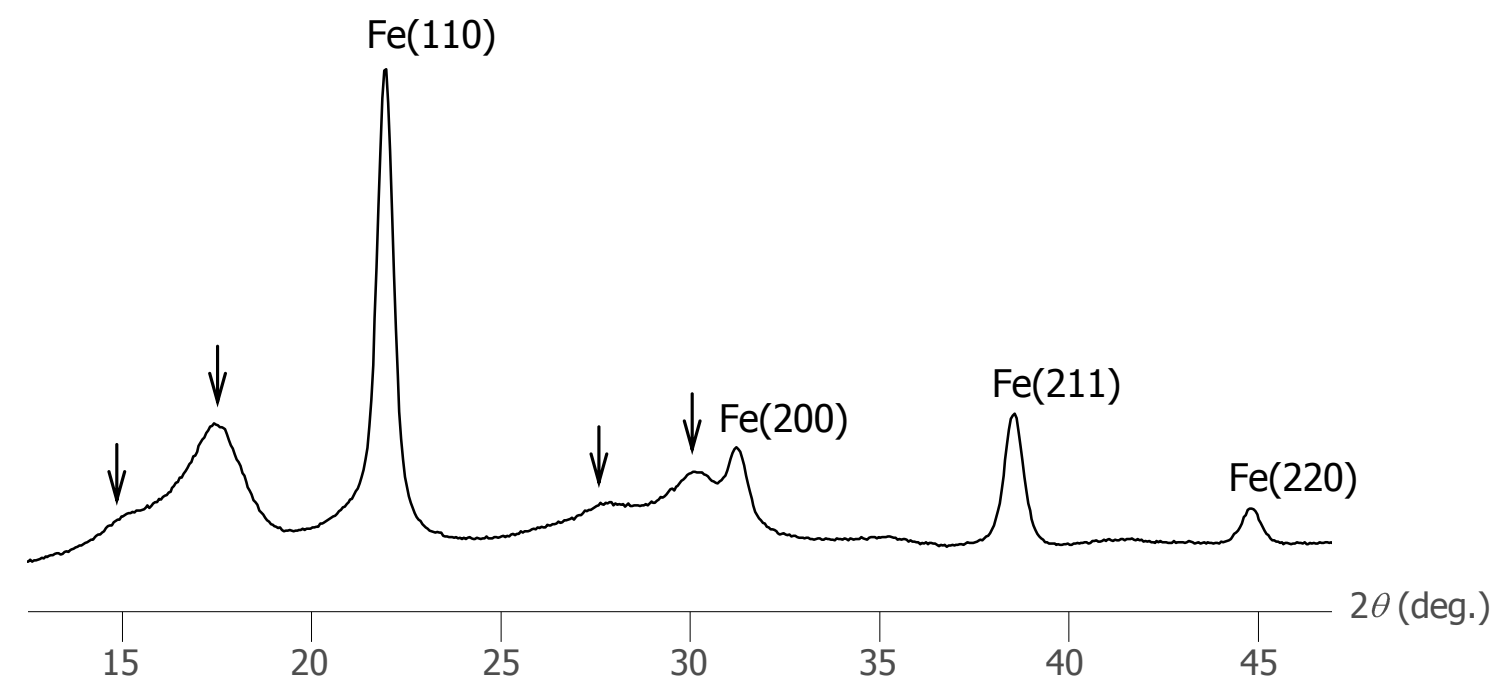

Figure 3-3. Powder XRD of sample Fe_B.

Relatively narrower and higher intensity peaks are indexed to $\alpha-\mathrm{Fe}$, the broader peaks (indicated by arrows) correspond to spinel iron oxide (220), (311), (511), and (440), from left to right, respectively.

An HRTEM image of a typical core/shell nanoparticle is shown in Figure 3-4a. The nanoparticle core, with a diameter of $\sim 8.2 \mathrm{~nm}$, was observed to be a single crystal of $\alpha$-Fe projected along a $\langle 100\rangle$ zone axis. The nanoparticle shell was observed to be iron oxide of polycrystalline structures. The shell thickness was $\sim 3.0 \mathrm{~nm}$.

Therefore, observations from XRD, SAED, and HRTEM were in good agreement. The nanoparticles obtained were near-monodisperse in size and shape, and comprised a core/shell structure, of which the core was a single crystal $\alpha$-Fe and the shell was spinel iron oxide of polycrystalline structures. 

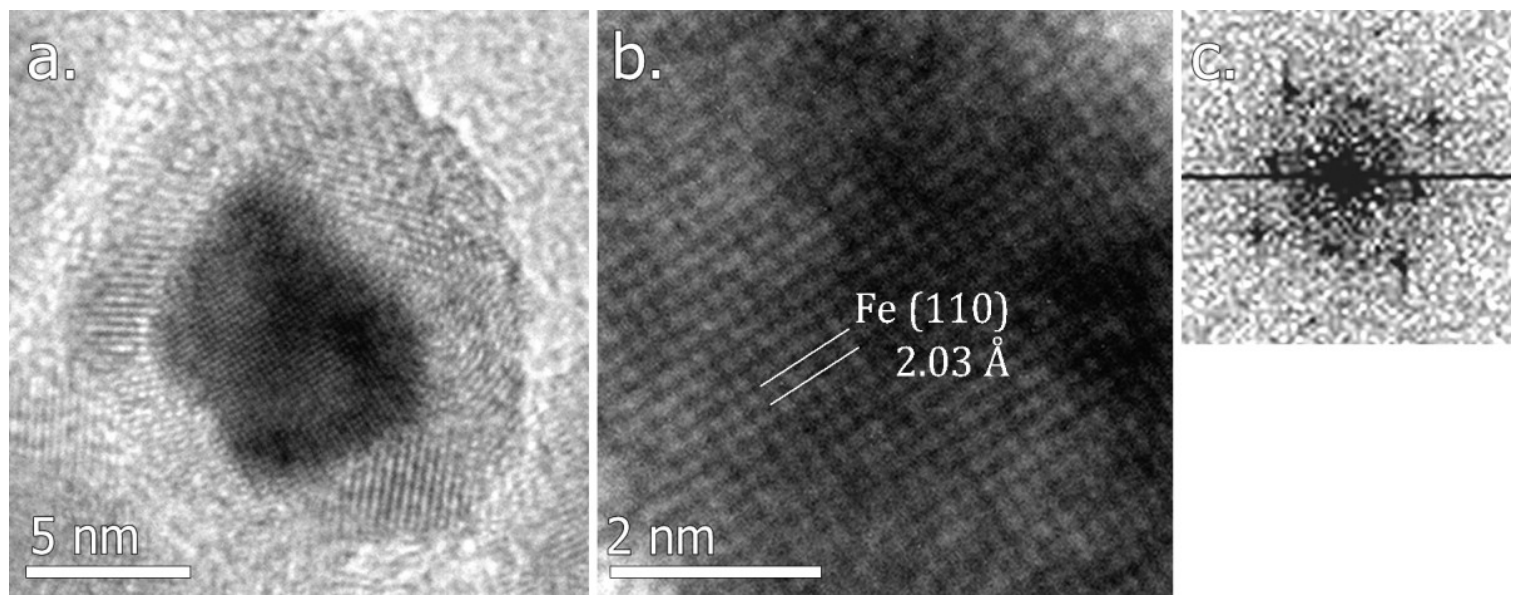

\section{Figure 3-4. Iron/iron oxide core/shell nanoparticle of sample Fe_B.}

a. HRTEM image of a typical core/shell nanoparticle. b. HRTEM image of the core of the nanoparticle, with power spectrum of the image shown in $\mathbf{c}$. The core of the nanoparticle is shown to be single crystal of cubic structure, being projected along a $\langle 100\rangle$ zone axis; lattice fringes corresponding to the Fe $\{110\}$ facets can be clearly observed in the HRTEM image.

The magnetic hysteresis loop of the sample measured at room temperature is shown in Figure 3-5a, with a close-up at the low field intervals shown in the inset. The nanoparticles exhibit ferromagnetic behaviour, with a remanence magnetisation, $M_{R}$ of $\sim 15 \mathrm{emu} \mathrm{g}^{-1}$, and a coercivity, $H_{c}$ of $\sim 370$ Oe. At the measured temperature, the sample did not saturate; the magnetisation, $M$ at an applied field, $H$ of $60 \mathrm{kOe}$ was measured to be $119.8 \mathrm{emu} \mathrm{g}^{-1}$. The data could be fitted with a linear function of $M$ against $H^{-1 / 2}$, which is plotted in Figure 3-5b. The saturation magnetisation, $M_{S}$ was determined to be $\sim 131 \mathrm{emu} \mathrm{g}^{-1}$, by extrapolating the plot to the intercept at $00^{-1 / 2}$. 

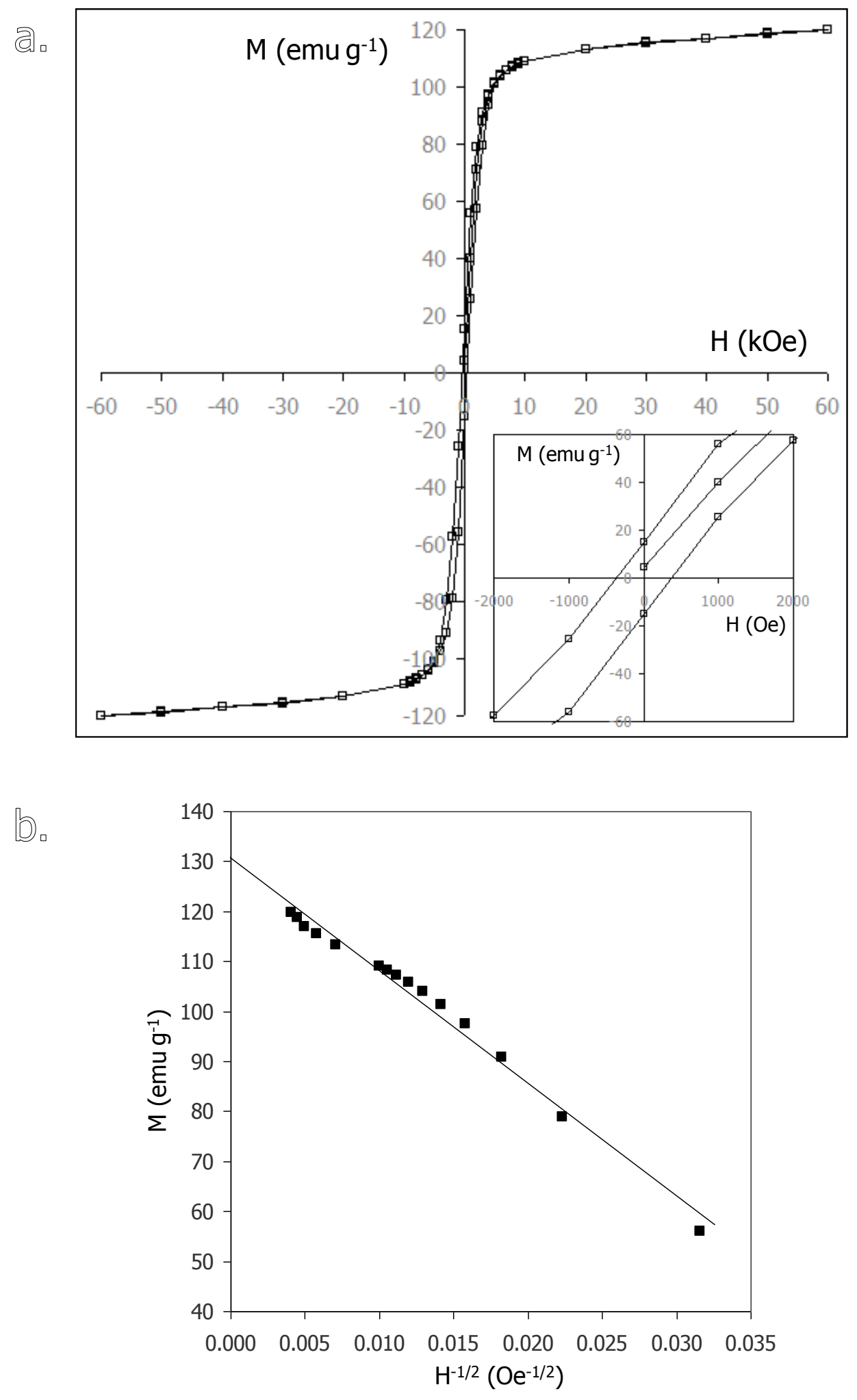

Figure 3-5. Magnetic properties of sample Fe_B at room temperature (300 K).

a. Plot of magnetisation, $\mathrm{M}$ against applied field, $\mathrm{H}$; inset shows plot at low field intervals. $\mathbf{b}$. The data was fitted with a linear function of $\mathrm{M}=-2252 \mathrm{H}^{-1 / 2}+131$ (shown by the solid line); $\mathrm{M}_{\mathrm{S}}$ was thus determined to be $\sim 131 \mathrm{emu} \mathrm{g}^{-1}$ (intercept at $0 \mathrm{Oe}^{-1 / 2}$ ). 


\section{Expt Fe_D. Reaction of $1.0 \mathrm{M}$ iron precursor in mesitylene (high concentration)}

When the precursor concentration was increased further to $1.0 \mathrm{M}$, a polydisperse sample was obtained. As shown in Figure 3-6, both core/shell and completely oxidised nanoparticles were observed, with nanoparticle size ranging from less than 5 to $\sim 30 \mathrm{~nm}$. Nanoparticle aggregates were also observed.

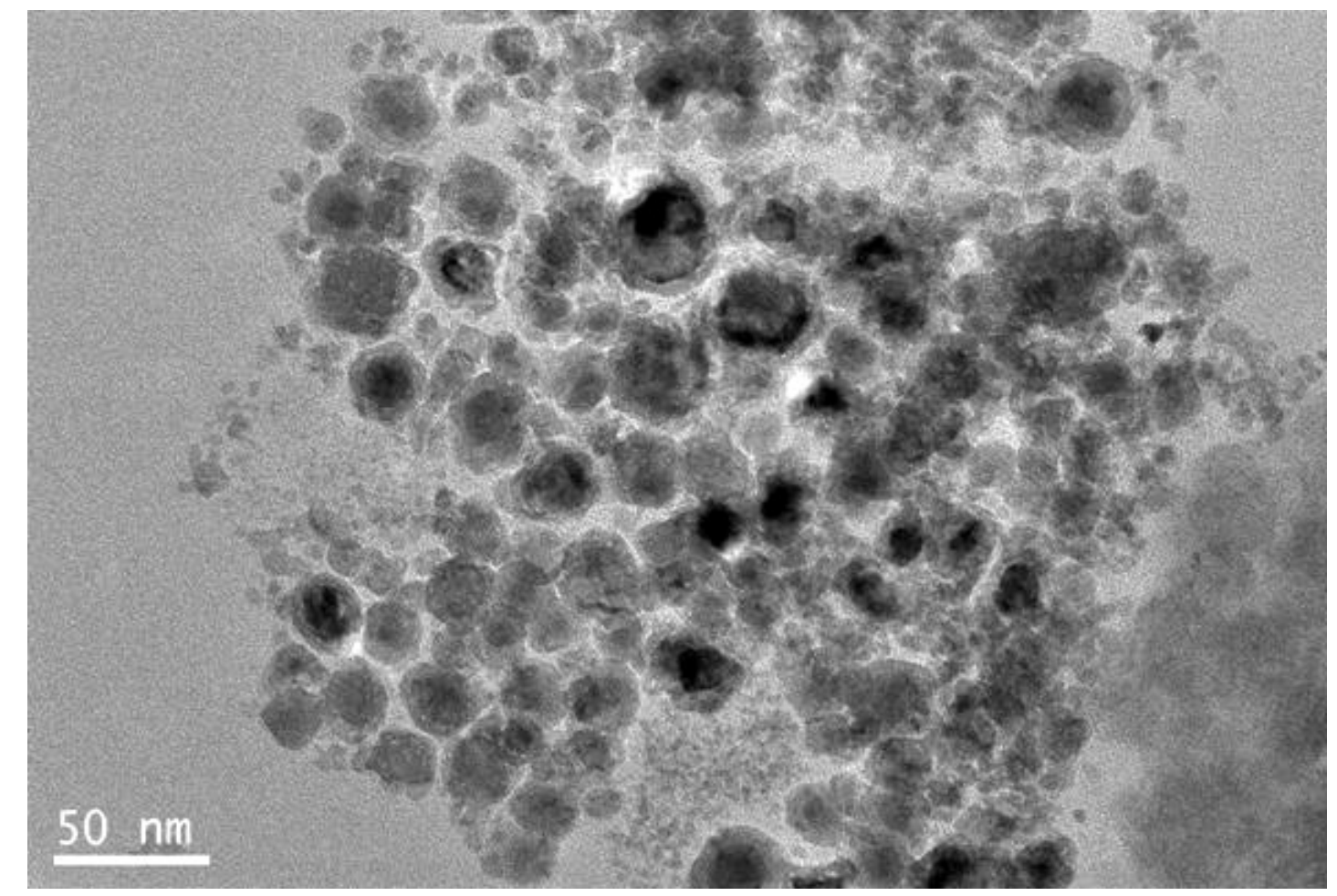

Figure 3-6. TEM image of nanoparticles from high-concentration reaction at $130{ }^{\circ} \mathrm{C}$ (Fe_D).

\subsubsection{Temperature}

The decomposition of the iron precursor was investigated at temperatures 130, 140, 150, and $160{ }^{\circ} \mathrm{C}$, with a precursor concentration of $0.25 \mathrm{M}$ and in the presence of 3 equivalents oleylamine.

Core/shell nanoparticles $>10 \mathrm{~nm}$ were obtained from reactions at temperatures 130 and $140{ }^{\circ} \mathrm{C}$. Nanoparticles obtained from reactions at 150 and $160{ }^{\circ} \mathrm{C}$ were $<10 \mathrm{~nm}$ in size and were of iron oxide structures. Within the temperature range investigated, nanoparticles 
observed in the different samples were spherical or near-spherical in shape. Hence the effect of temperature on nanoparticle shape was not significant, compared to the influence on nanoparticle size.

\subsubsection{Stabilising Agent}

The use of oleylamine as the stabilising agent in this study was investigated by varying the mole ratio of oleylamine to iron at $130^{\circ} \mathrm{C}$. Reactions with oleylamine/iron $=0.2,1,3$, and 6 were carried out with precursor solutions of $0.25 \mathrm{M}$ in mesitylene.

Reactions with the use of 1,3 , and 6 equivalents oleylamine produced core/shell nanoparticles of similar sizes, indicating the amount of oleylamine within this range at $130{ }^{\circ} \mathrm{C}$ had no effect on the nanoparticles obtained. When the relative concentration of oleylamine was low, at 0.2 equivalents, completely oxidised nanoparticles were obtained.

Expt Fe_B $\left(0.25 \mathrm{M}, 130^{\circ} \mathrm{C}\right)$ was repeated with 3 equivalents oleic acid as the stabiliser and no precipitate was observed after 3 days. The colour of the reaction solution was reddish brown. The same result was obtained when a mixture of oleylamine and oleic acid was used, with mole ratio iron : oleylamine : oleic acid $=1: 1.5: 1.5$. It is believed that the oleic acid might have complexed the precursor, resulting in a stabilised precursor that could not be decomposed at the temperature employed.

\subsubsection{Other Variables}

Time

Reactions at $130-140{ }^{\circ} \mathrm{C}$ were terminated after 2 days, which was found to produce optimum results, in which the nanoparticles obtained had grown to a thermodynamically stable morphology and size under the reaction conditions. For experimental conditions that produced core/shell nanoparticles, polydisperse samples were usually observed when the experiment was terminated 1 day shorter or longer than the average time. When an experiment was terminated 1 day earlier, the nanoparticles obtained were mostly smaller than those obtained at the average time. When the nanoparticles were collected more than 1 day after the average time, nanoparticles of different morphologies were observed, including spherical, rod-like, and various irregular structures. While the spherical or 
near-spherical nanoparticles oxidised on the surface, the non-spherical ones oxidised completely to form iron oxide nanoparticles.

At temperatures 150 and $160{ }^{\circ} \mathrm{C}$, nanoparticles of 5-8 nm were obtained after 1-2 hours. When the same reaction was left overnight for 12-20 hours, chain-like nanostructures were formed as a result of nanoparticle coalescence.

\section{Pressure}

Experiments Fe_B $\left(0.25 \mathrm{M}, 3\right.$ equivalents oleylamine, at $\left.130{ }^{\circ} \mathrm{C}\right)$ and $\mathrm{Fe} \_\mathrm{G}(0.25 \mathrm{M}$, 3 equivalents oleylamine, at $160{ }^{\circ} \mathrm{C}$ ) were repeated under 300 and $200 \mathrm{kPa} \mathrm{H}_{2}$, respectively. Results were the same as those carried out with an initial pressure of $100 \mathrm{kPa} \mathrm{H}_{2}$.

\subsubsection{Formation of Iron Oxide Nanoparticles}

Iron oxide nanoparticles of spinel phases - magnetite $\left(\mathrm{Fe}_{3} \mathrm{O}_{4}\right)$ or maghemite $\left(\gamma-\mathrm{Fe}_{2} \mathrm{O}_{3}\right)$, or both - were observed in many samples. It is possible that the oxide nanoparticles were formed during the synthesis, or were a result of complete oxidation of iron nanoparticles. In order to find out which path led to the formation of these iron oxide nanoparticles, the reaction at $160^{\circ} \mathrm{C}\left(\mathrm{Fe}_{-} \mathrm{G}\right)$ was repeated and purification was conducted under a nitrogen atmosphere.

Figure 3-7a shows a TEM image of the nanoparticles purified under nitrogen, which were of core/shell structures. The overall nanoparticle morphology and size were the same as that observed in the sample purified in air, which were similar to those in Figure 3-1a. Aggregates were also observed in the sample. The SAED pattern revealed both $\alpha$-Fe and spinel iron oxide structures in the sample, in contrast to a pure iron oxide structure for the sample purified in air (see SAED pattern in Figure 3-1b). The observations thus indicated that $\alpha$-Fe nanoparticles of $\leq 8 \mathrm{~nm}$ were originally formed in the synthesis and then oxidised completely upon exposure to air. 


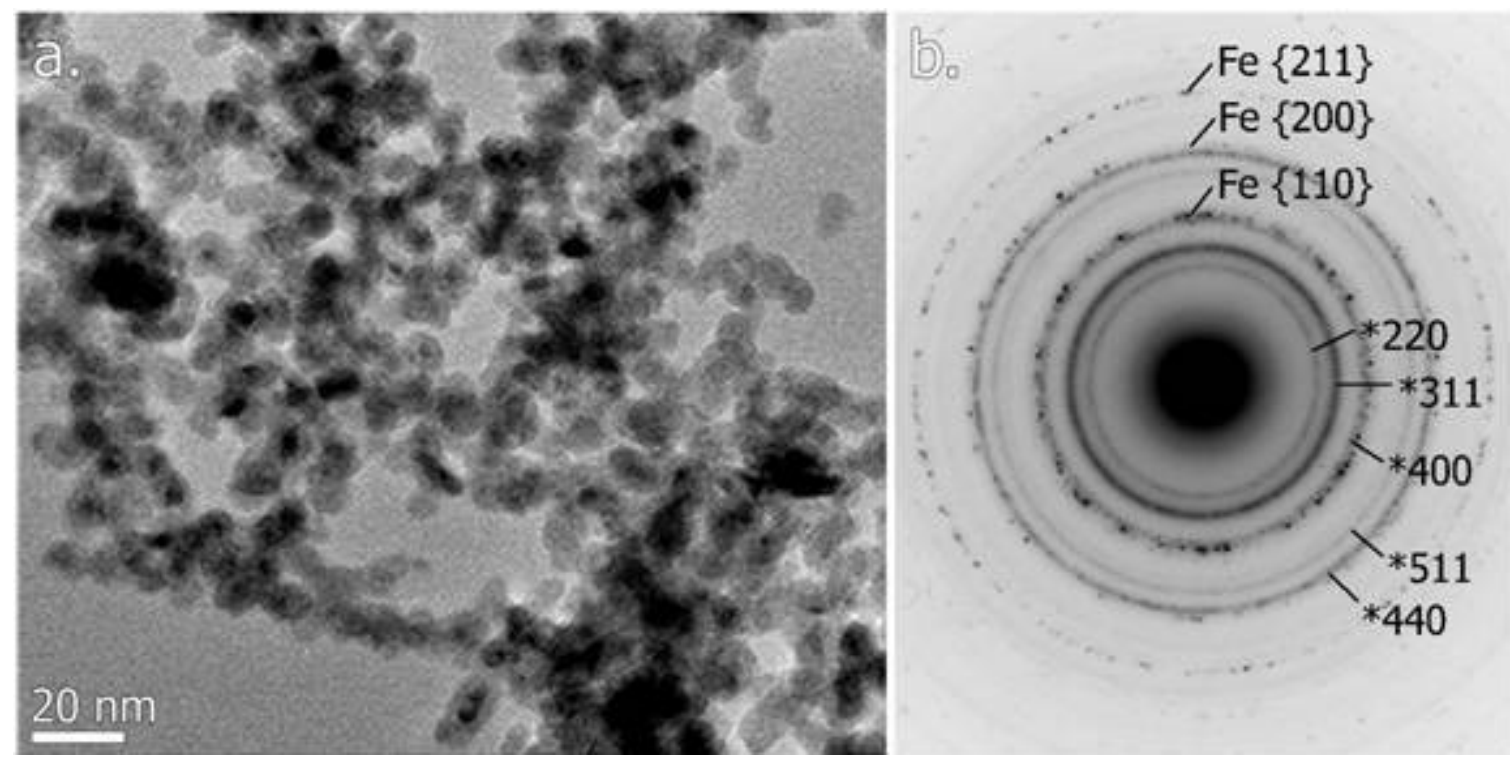

Figure 3-7. TEM and SAED results of sample purified under a nitrogen atmosphere.

a. TEM image of core/shell nanoparticles collected after purification under nitrogen. b. SAED showing diffraction rings corresponding to $\alpha$-Fe in addition to those of spinel iron oxide (*).

\subsection{Bench-Top Synthesis}

Synthesis of iron nanoparticles using bench-top Schlenk line technique was carried out in a three-necked round bottom flask under a nitrogen atmosphere. Two experiments were conducted, with experimental conditions listed in Table 3-4. Expt Fe_L was conducted at $180^{\circ} \mathrm{C}$ to investigate the decomposition of the iron precursor at a higher temperature than those employed in the Fischer-Porter bottle synthesis. The reaction was repeated at $160{ }^{\circ} \mathrm{C}$ (Expt Fe_M).

Table 3-4. Summary table of bench-top synthesis.

\begin{tabular}{|c|c|c|c|c|l|c|}
\hline Expt & $\begin{array}{c}\text { Conc. }^{\mathrm{A}} \\
(\mathbf{M})\end{array}$ & OLA $^{\mathrm{B}} / \mathrm{Fe}$ & $\begin{array}{c}\text { Temp. } \\
\left.\mathbf{}^{\mathbf{C}} \mathbf{C}\right)\end{array}$ & Time & Nanoparticles & $\begin{array}{c}\text { Avg. size } \\
(\mathbf{n m})\end{array}$ \\
\hline $\mathrm{Fe}_{-} \mathrm{L}$ & 0.16 & 5 & 180 & $2 \mathrm{~h}$ & Core/shell, faceted & $53 \pm 4$ \\
\hline $\mathrm{Fe}_{-} \mathrm{M}$ & 0.16 & 5 & 160 & $2 \mathrm{~h}$ & Core/shell, sponge-like & $65 \pm 15$ \\
\hline
\end{tabular}

A dichlorobenzene was used as the solvent.

в OLA = oleylamine 


\section{Expt Fe_L. Reaction at $180{ }^{\circ} \mathrm{C}$}

A black precipitate was collected after purification by magnetic separation. The precipitate was observed to react strongly to the magnet during purification. As shown in Figure 3-8a, highly faceted, cube-like, core/shell nanoparticles were obtained, with an average size of $53 \pm 4 \mathrm{~nm}$. In the inset, the SAED pattern acquired from a large ensemble of nanoparticles showed the presence of $\alpha$-Fe and iron oxide of spinel structures. The appearance of high-intensity diffraction spots correspond to $\alpha$-Fe and much weaker diffraction rings to iron oxide indicated large $\alpha$-Fe crystallite and small or polycrystalline structure of the iron oxide. HRTEM imaging of individual nanoparticles showed that the core was of single crystal $\alpha$-Fe and the shell was iron oxide of polycrystalline structure. A typical faceted core/shell nanoparticle is shown in Figure 3-8b. The power spectrum acquired from the core area of the image (marked with a white square) is shown in Figure 3-8c, which shows that the faceted core was viewed along a $\langle 100\rangle$ zone axis of $\alpha$-Fe. 

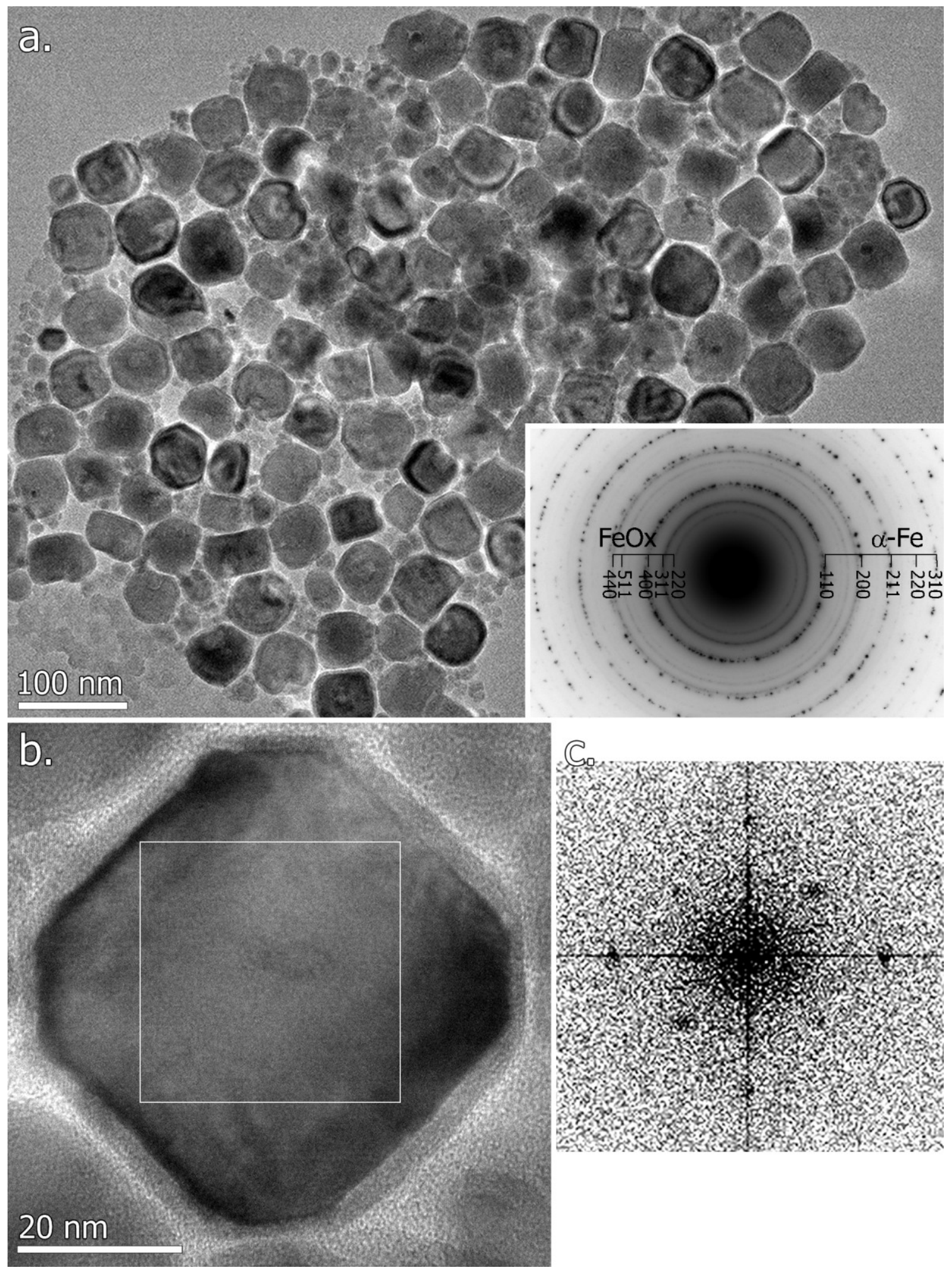

Figure 3-8. TEM and SAED results of bench-top synthesis at $180^{\circ} \mathrm{C}\left(\mathrm{Fe} \_\mathrm{L}\right)$.

a. TEM image of nanoparticles obtained, typically faceted and $\sim 55 \mathrm{~nm}$ in size; inset shows SAED pattern indexed to $\alpha$-Fe and spinel iron oxide. $\mathbf{b}$. TEM image of a typical faceted, cube-like, core/shell nanoparticle. c. Power spectrum of the core area of image b (indicated by white square), which can be matched to a $\langle 100\rangle$ projection of a cubic structure. The core of the nanoparticles is thus a single crystal $\alpha$-Fe projected along a $\langle 100\rangle$ zone axis, and is terminated with $\{100\}$ and $\{110\}$ facets. 


\section{Expt Fe_M. Reaction at $160{ }^{\circ} \mathrm{C}$}

The exact conditions and procedure in Expt Fe_L were repeated at $160{ }^{\circ} \mathrm{C}$. A black precipitate that responded strongly to a magnet was collected. As shown in Figure 3-9a, the nanoparticles obtained were near-spherical in shape, with a relatively large size distribution of 50-80 $\mathrm{nm}$. The nanoparticle morphology was observed to resemble that of a sponge, with very uneven surfaces; these nanoparticles are thus described as sponge-like nanoparticles.

The fact that the nanoparticles had very uneven surfaces might suggest nanoparticle aggregation or agglomeration. However, close examination on individual nanoparticles revealed otherwise. A TEM image of a typical sponge-like nanoparticle is shown in Figure 3-10a. Figure 3-10b shows the SAED pattern acquired from an area encircling the whole nanoparticle. The diffraction pattern was indexed to an $\alpha$-Fe being projected along a $\langle 100\rangle$ zone axis, showing single-crystal structure of the whole nanoparticle. This observation thus ruled out the indication that the sponge-like nanoparticle could be an aggregate of smaller nanoparticles.
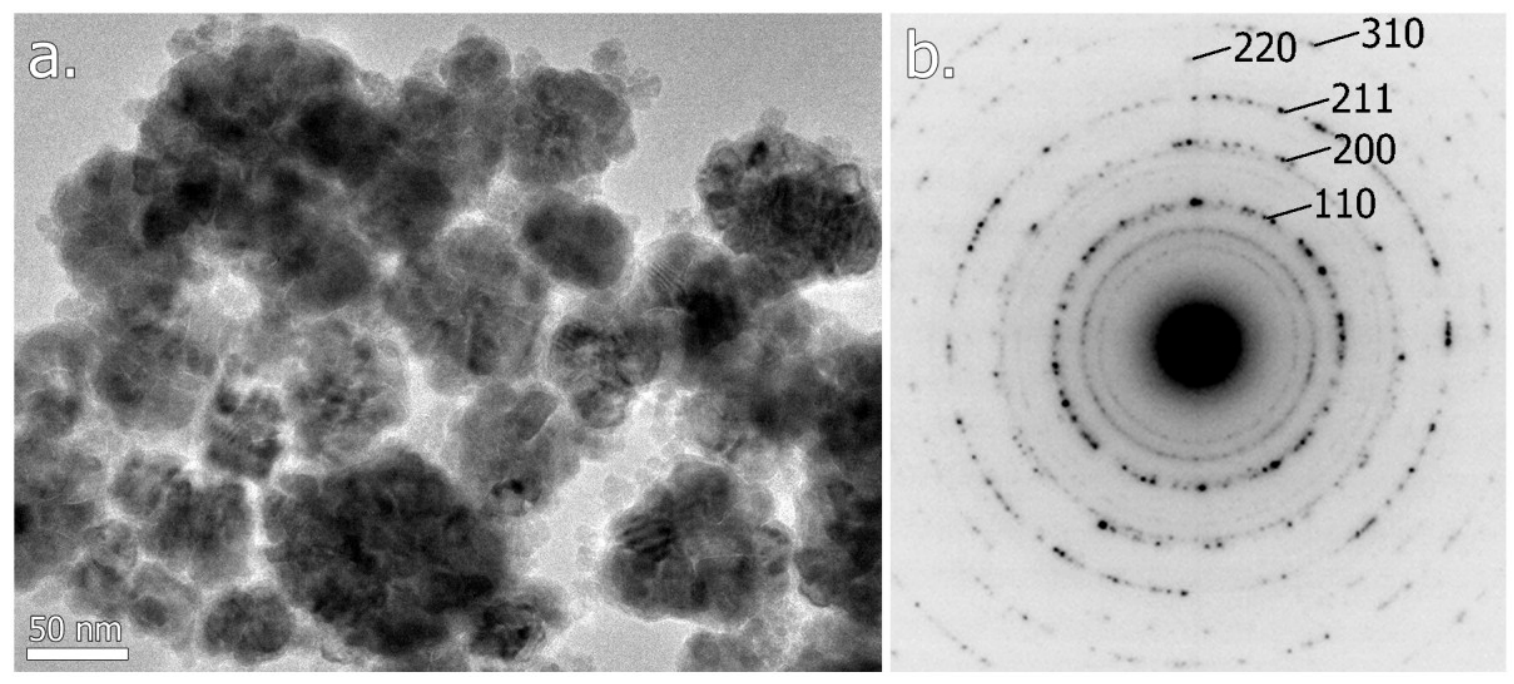

Figure 3-9. TEM and SAED results from bench-top synthesis at $160{ }^{\circ} \mathrm{C}(\mathrm{Fe}-\mathrm{M})$.

a. TEM image of the nanoparticles obtained. b. SAED pattern with diffraction rings indexed to $\alpha$ - $\mathrm{Fe}$, the other weaker diffraction rings correspond to the spinel iron oxide. 


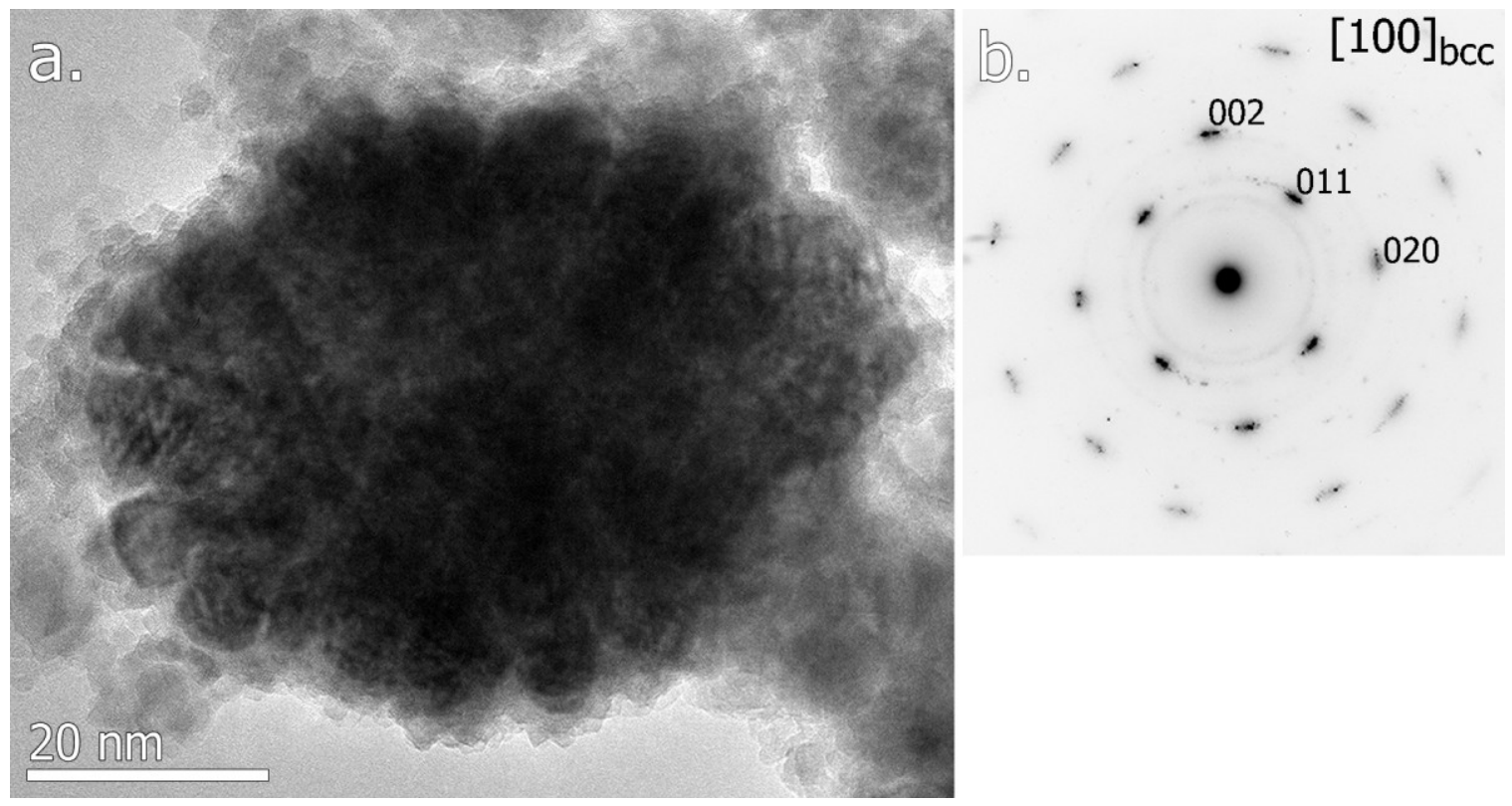

Figure 3-10. A typical sponge-like nanoparticle.

a. TEM image of a typical sponge-like nanoparticle. b. SAED pattern acquired from an area encircling the nanoparticle, which can be indexed to a $\langle 100\rangle$ projection of $\alpha$-Fe.

\subsection{Discussion}

\subsubsection{Nanoparticle Composition}

In this study, iron nanoparticles of $>8 \mathrm{~nm}$ were observed to oxidise on the surface to form iron/iron oxide core/shell nanoparticles. As oxidation had occurred on the surface of the nanoparticles, the crystal structure of the core should be the as-synthesised structure. Based on this reasoning, nanoparticles of single-crystal $\alpha$-Fe, as observed for the nanoparticle core in different samples, were obtained from different syntheses.

The iron nanoparticles were synthesised through the decomposition of the iron precursor $\mathrm{Fe}\left(\mathrm{C}_{5} \mathrm{H}_{5}\right)\left(\mathrm{C}_{6} \mathrm{H}_{7}\right)$ at temperatures $130-180^{\circ} \mathrm{C}$. Under a hydrogen or nitrogen atmosphere, the precursor decomposes to yield free iron atoms, which would then nucleate and grow into $\alpha$-Fe nanocrystals. The hydrocarbon part of the precursor naturally becomes the by-product of the reaction, and could be separated during purification, or evaporated off the solution during or after the experiment. The proposed pathway leading to the formation of $\alpha$-Fe nanoparticles as described is illustrated in Scheme 3-1. 


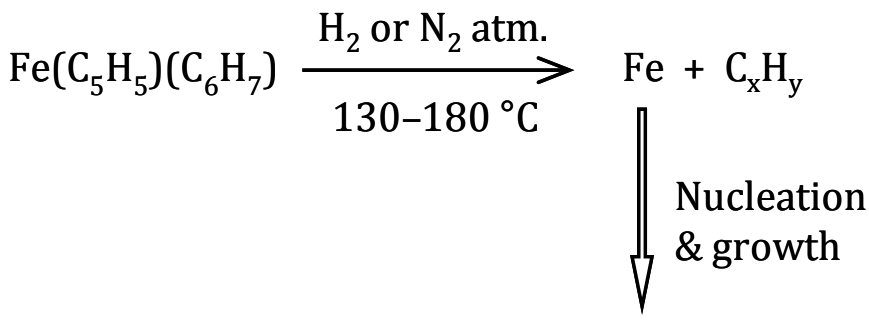

$$
\begin{aligned}
& \alpha \text {-Fe NPs }
\end{aligned}
$$

\section{Scheme 3-1. Proposed pathway for the formation of iron nanoparticles.}

For core/shell nanoparticles, the iron oxide shell was observed to be polycrystalline. The multi-domain orientations of the iron oxide shell are believed to be a result of the uncontrolled oxidation process that took place when the iron nanocrystals were exposed to air. By oxidising the as-synthesised iron nanoparticles in a controlled fashion, Peng et al. showed that the crystallinity of oxide shell could be influenced by the oxidation process.[16]

\subsubsection{Nanoparticle Size}

Nanoparticles obtained from Fischer-Porter bottle syntheses were mainly spherical or near-spherical in morphology. Generally two sizes were observed among the different spherical nanoparticles collected, mainly dependent on the reaction temperature. Reactions at lower temperatures $\left(130-140^{\circ} \mathrm{C}\right)$ produced nanoparticles of $\sim 14 \mathrm{~nm}$ in size, while those at higher temperatures $\left(150-160^{\circ} \mathrm{C}\right)$ yielded nanoparticles of $\sim 6 \mathrm{~nm}$. The same trend was also observed in the bench-top synthesis, in which nanoparticles formed at $160{ }^{\circ} \mathrm{C}$ were generally larger than those obtained at $180{ }^{\circ} \mathrm{C}$.

At higher temperature, the decomposition rate of the precursor increases, producing a high concentration of free iron atoms, and results in more nuclei being formed. As relatively more precursor is consumed for nucleation, less remained for growth, therefore yielding smaller-sized nanoparticles. Growth of 14-nm nanoparticles was favoured at $130-140{ }^{\circ} \mathrm{C}$. At this temperature range, the nanoparticle size was observed to be influenced by both precursor concentration and the amount of oleylamine used, as summarised in Table 3-5.

For different reactions at the same temperature, the rates of precursor decomposition and nanoparticle formation and growth are expected to be dependent on the concentration of 
the reactants. In this study, $0.25 \mathrm{M}$ was observed to be the ideal precursor concentration for controlled growth of nanoparticles, producing near-monodisperse nanoparticles of $\sim 14 \mathrm{~nm}$ in size. Although larger nanoparticles were observed in the sample from the high-concentration reaction, the nanoparticle size distribution was also large. This suggests that when precursor concentration is high, growth becomes less controlled leading to a polydisperse sample. At low precursor concentration, the condition is believed to be similar to that of the high-temperature reaction, where there is limited precursor available for nanoparticle growth. It might be possible that monodisperse nanoparticles of $>14 \mathrm{~nm}$ can be achieved by tuning the concentration between the middle $(0.25 \mathrm{M})$ and high $(1.0 \mathrm{M})$ concentrations.

The role of oleylamine is apparent when the amount used is considerably less, at 0.2 equivalents. Nanoparticles formed under this condition were $6.5 \mathrm{~nm}$ in average, much smaller than those obtained from reactions with 3 equivalents of oleylamine. Different studies have revealed that the interactions of stabilising molecules with the precursor and/or reaction intermediate could influence the reaction pathways through to nucleation and then growth of nanoparticles. ${ }^{[17,18]}$ It is thus believed that a minimal oleylamine to iron ratio is required for effective interactions with the precursor and/or reaction intermediate, to prevent too much precursor being consumed for nucleation and not enough left for growth.

Table 3-5. Combined effect of precursor concentration and amount of oleylamine on nanoparticle size.

\begin{tabular}{|l|c|c|c|c|}
\hline $\begin{array}{l}\text { OLA*/Fe } \\
\begin{array}{l}\text { Conc. } \\
\text { (M) }\end{array}\end{array}$ & $\mathbf{0 . 2}$ & $\mathbf{1}$ & $\mathbf{3}$ & $\mathbf{6}$ \\
\hline $\mathbf{0 . 0 5}$ & - & - & $7 \pm 2$ & - \\
\hline $\mathbf{0 . 2 5}$ & $6 \pm 3$ & $14 \pm 2$ & $14 \pm 2$ & $16 \pm 5$ \\
\hline $\mathbf{1 . 0}$ & - & - & $3-30$ & - \\
\hline * OLA = oleylamine & & & \\
\hline
\end{tabular}

\subsubsection{Stability of Nanoparticles}

The average oxide shell thickness was observed to be around $3 \mathrm{~nm}$, independent of the nanoparticle size, which is consistent with observations in the literature.[3] The core/shell 
nanoparticles in Expt Fe_B were observed to be stable for at least 1 month. After 1 year, voids were clearly observed at the interface between the core and the shell for some of the nanoparticles, typically those of $15 \mathrm{~nm}$ and smaller, as shown in Figure 3-11b. This suggests that oxidation continued to take place, thus indicating ineffective passivation of the oxide shell over time. For nanoparticles of larger than $15 \mathrm{~nm}$, the presence of void was less visible. It is believed that further oxidation continued to occur for all core/shell nanoparticles. For larger nanoparticles, it is likely that the resulting vacancies are much smaller in volume relative to the nanoparticle size and hence were not obvious when viewed in the TEM.
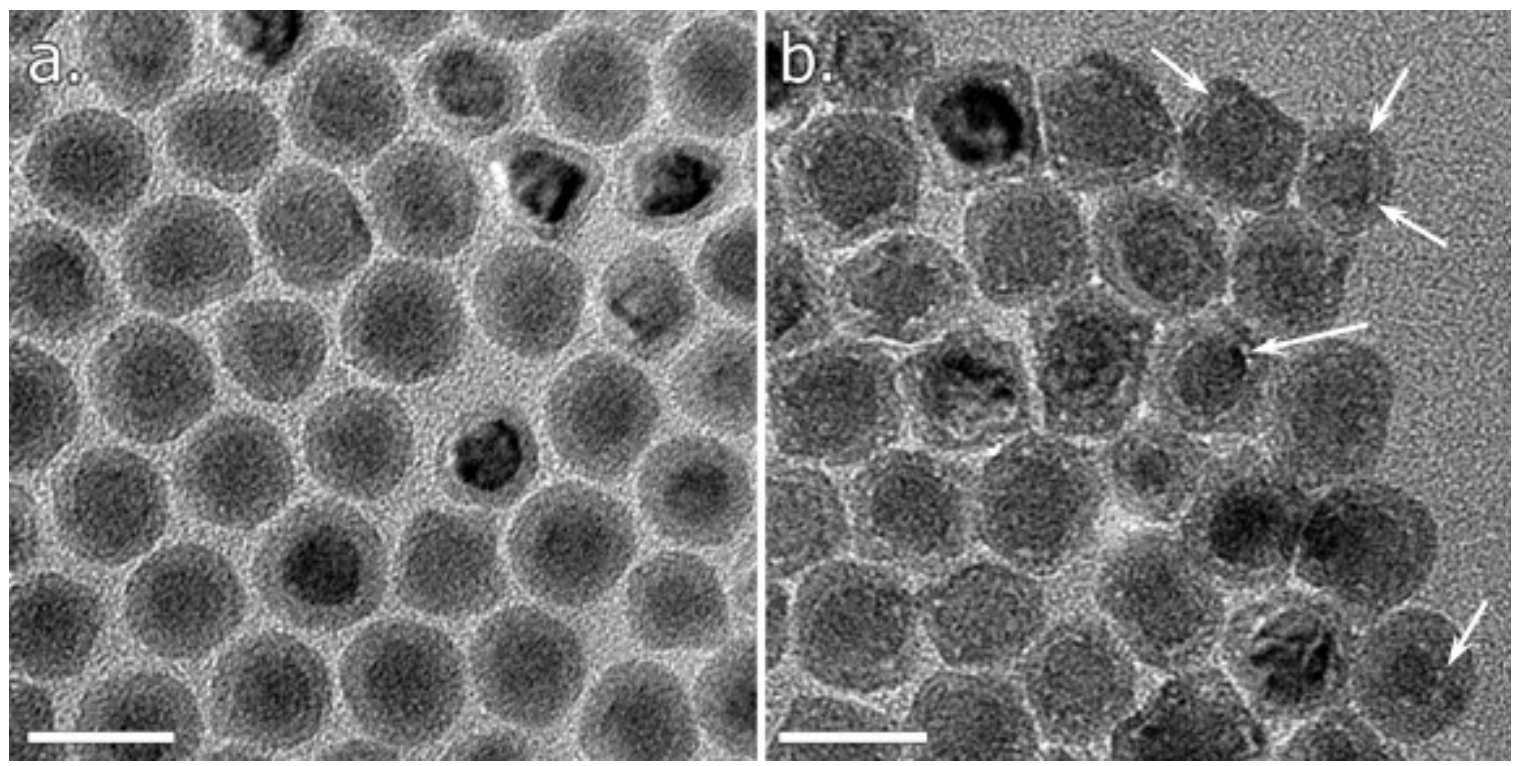

Figure 3-11. Stability of core/shell nanoparticles of $\sim \mathbf{1 4} \mathbf{~ n m}$.

a. TEM image of core/shell nanoparticles in Expt Fe_B, taken after 1 month of synthesis. b. TEM image of the same sample after 1 year, showing presence of voids at the interface between the core and shell for most of the nanoparticles. Arrows highlight the clearly visible voids in some of the nanoparticles. All scale bars are $20 \mathrm{~nm}$.

For the $6 \mathrm{~nm}$ nanoparticles that were purified in nitrogen (see Section 3.2.5), after 1 year the nanoparticles had turned hollow and the corresponding SAED revealed the presence of only iron oxide in the sample, as shown in Figure 3-12b. The formation of vacancies within nanoparticles leading to hollow nanostructures has been observed for different materials including iron nanoparticles. ${ }^{[3,19]}$ It has been widely accepted that the nanoscale Kirkendall effect $^{[20]}$ is responsible for this phenomenon. In this case, void or vacancy is generated as the outward diffusion of iron atoms occurs at a higher rate than the inward diffusion of oxygen atoms during the continuous oxidation process. Hollow nanostructures are formed as more vacancies are produced and ultimately coalesce into a single void. 

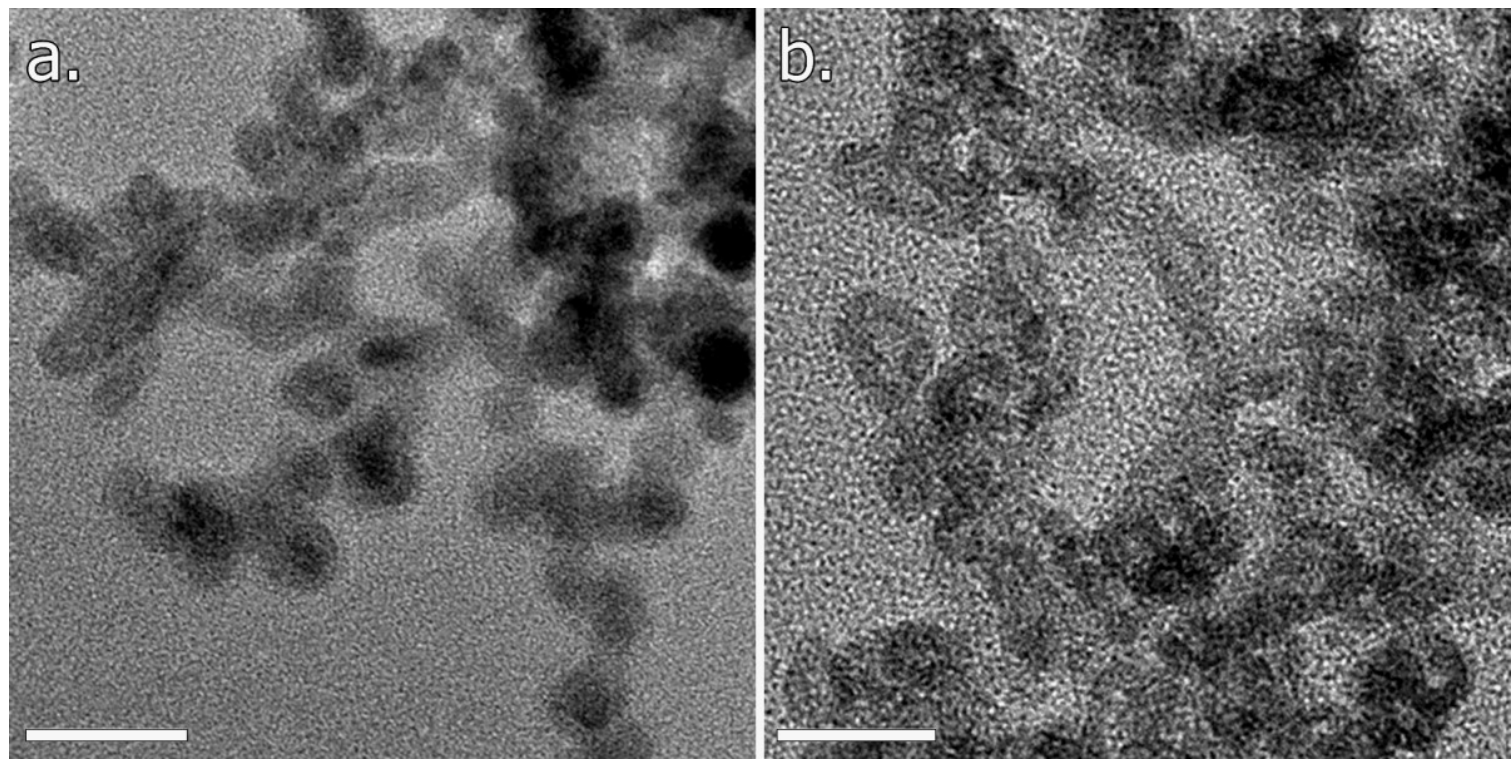

Figure 3-12. Stability of core/shell nanoparticles of $\sim 7 \mathbf{~ n m}$.

a. TEM image of $7 \mathrm{~nm}$ core/shell nanoparticles purified under nitrogen (Fe_G). b. TEM image of the same sample after 1 year, showing that the nanoparticles had become hollow. All scale bars are $20 \mathrm{~nm}$.

\subsubsection{Magnetic Properties}

The 14-nm iron/iron oxide core/shell nanoparticles were shown to exhibit ferromagnetic behaviour at room temperature, and a saturation magnetisation, $M_{S}$ of $131 \mathrm{emug}^{-1}$ was determined. The $M_{S}$ obtained is much greater than that of bulk iron oxide (82 and $70 \mathrm{emu} \mathrm{g}^{-1}$ for $\mathrm{Fe}_{3} \mathrm{O}_{4}$ and $\gamma-\mathrm{Fe}_{2} \mathrm{O}_{3}$, respectively), which is ferrimagnetic at room temperature and is expected to be superparamagnetic in the nanometre scale.[21] The high $M_{S}$ value and the ferromagnetic behaviour suggest that these properties are largely attributed to the iron core. Furthermore, ferromagnetic behaviour is generally expected for randomly distributed spherical single domains of iron metal particles.[1]

The magnetic properties obtained at room temperature are compared with those reported for iron nanoparticles and iron/iron oxide core/shell nanoparticles of similar sizes, in Table 3-6. Both the $M_{S}$ and $H_{C}$ of sample Fe_B are larger than the others. The slightly enhanced magnetic properties could be explained by the relatively larger nanoparticle size, an effect that is observed for magnetic materials within the single-domain regime.[6] Although the nanoparticles reported by Griffiths et al.[22] are larger in size, the respective $M_{S}$ 
and $H_{C}$ values are smaller, compared with those of sample Fe_B. As the former was stabilised by polymer molecules which are much bulkier than oleylamine, the magnetic interactions between the nanoparticles, i.e. the magnetic domains, are believed to be weakened comparatively. It was also reported by Bomati-Miguel et al.[4] that the as-prepared, ferromagnetic nanoparticles became superparamagnetic after the nanoparticles were coated with polymers. This thus implies that the iron/iron oxide core/shell nanoparticles prepared in this study have the potential to be modified on the surfaces to exhibit ferro- or superparamagnetic behaviour, suited to the requirements of the respective applications.

Table 3-6. Comparison of magnetic properties of similar nanoparticles.

\begin{tabular}{|c|c|c|}
\hline Reference & Nanoparticles & $\begin{array}{l}\text { Magnetic properties at } \\
\text { room temperature }\end{array}$ \\
\hline Griffiths et al.[22] & 16-nm $\alpha$-Fe NPs stabilised by polymer & $\begin{array}{l}M_{S}=130 \mathrm{emu} \mathrm{g}^{-1} \\
H_{C}=150 \mathrm{Oe}\end{array}$ \\
\hline Suslick et al.[12] & 8-nm $\alpha$-Fe NPs stabilised by polymer & $\begin{array}{l}M_{S}=101 \mathrm{emu} \mathrm{g}^{-1} \\
H_{C}=00 \mathrm{e}\end{array}$ \\
\hline Carpenter et al.[23] & $\begin{array}{l}\text { 11.5-nm core/shell NPs stabilised by polymer; } \\
\alpha \text {-Fe core, oxide shell thickness } 2.7 \mathrm{~nm}\end{array}$ & $\begin{array}{l}M_{S}=106 \mathrm{emu} \mathrm{g}^{-1} \\
H_{C}<100 \mathrm{Oe}\end{array}$ \\
\hline Farrell et al.[24] & $\begin{array}{l}\text { 11.2-nm core/shell NPs stabilised by } \\
\text { oleylamine-oleic acid mixture, oxide shell } \\
\text { thickness } 2.1 \mathrm{~nm}\end{array}$ & $\begin{array}{l}M_{S}(\text { at } 10 \mathrm{~K})=132 \mathrm{emu} \mathrm{g}^{-1} \\
H_{C} \text { not reported }\end{array}$ \\
\hline Bomati-Miguel et al. ${ }^{[4]}$ & $\begin{array}{l}\text { 12-nm core/shell NPs; } \\
\alpha \text {-Fe core, oxide shell thickness } 3-4 \mathrm{~nm}\end{array}$ & $\begin{array}{l}M_{S}=85 \mathrm{emu} \mathrm{g}^{-1} \\
H_{C}=250 \mathrm{Oe}\end{array}$ \\
\hline Expt Fe_B & $\begin{array}{l}\text { 14-nm core/shell NPs stabilised by oleylamine; } \\
\alpha \text {-Fe core, oxide shell thickness } 2.6 \mathrm{~nm}\end{array}$ & $\begin{array}{l}M_{S}=131 \mathrm{emu} \mathrm{g}^{-1} \\
H_{C}=370 \mathrm{Oe}\end{array}$ \\
\hline
\end{tabular}

\subsection{Chapter Summary}

In this study, single crystal $\alpha$-Fe nanoparticles were successfully synthesised via a facile chemical route through the decomposition of the precursor $\mathrm{Fe}\left(\mathrm{C}_{5} \mathrm{H}_{5}\right)\left(\mathrm{C}_{6} \mathrm{H}_{7}\right)$, by employing the Fischer-Porter bottle and bench-top Schlenk line techniques. Nanoparticles of $>8 \mathrm{~nm}$ oxidised on the surface to form iron/iron oxide core/shell nanoparticles, and were stable 
for at least 1 month. A saturation magnetisation of $130.7 \mathrm{emug}^{-1}$ was obtained for core/shell nanoparticles of $\sim 14 \mathrm{~nm}$.

Further oxidation of the core/shell structures continued to take place over time (in 1 year), leading to the formation of voids at the interface between the iron core and the oxide shell of nanoparticles. Voids were clearly visible for nanoparticles of $15 \mathrm{~nm}$ and smaller.

The synthesis was investigated with different experimental conditions. Spherical and near-spherical nanoparticles were synthesised in Fischer-Porter bottles at $130-160{ }^{\circ} \mathrm{C}$. Nanoparticle size was found to be controlled by precursor concentration, reaction temperature and the amount of oleylamine (stabilising agent) added. Highly faceted nanoparticles were obtained from the bench-top method at $180^{\circ} \mathrm{C}$.

\subsection{Experimental}

\section{Materials}

The iron precursor Fe $\left(\mathrm{C}_{5} \mathrm{H}_{5}\right)\left(\mathrm{C}_{6} \mathrm{H}_{7}\right)$ was prepared according to the method described in the literature.[25] Mesitylene (98\%) and 1,2-dichlorobenzene (99\%) were purchased from Sigma-Aldrich; and oleylamine (technical, $\geq 70 \%$ ) was purchased from Fluka. Toluene was of reagent grade. All reagents were used as received without further purification, unless otherwise specified.

\section{Fischer-Porter bottle synthesis}

For a typical Fisher-Porter bottle experiment, $0.08 \mathrm{~g}(\sim 0.4 \mathrm{mmol})$ of the iron precursor $\mathrm{Fe}\left(\mathrm{C}_{5} \mathrm{H}_{5}\right)\left(\mathrm{C}_{6} \mathrm{H}_{7}\right)$ was weighed in a vial, then added with mesitylene as the solvent and oleylamine as the stabiliser. The vial was placed in a Fischer-Porter bottle, which was then flushed 3 times with hydrogen gas $\left(\mathrm{H}_{2}\right)$ before being filled with $100-300 \mathrm{kPa} \mathrm{H}_{2}$, and sealed. The bottle was placed into an oven heated at a reaction temperature between 130 and $160^{\circ} \mathrm{C}$, and remained for 2 days.

\section{Bench-top Schlenk line synthesis}

In a three-necked round bottom flask fitted with a water condenser, a thermocouple, and a septum, $8 \mathrm{~mL}$ of dichlorobenzene was degassed for 30 minutes, and heated to and 
maintained at 160 or $180^{\circ} \mathrm{C}$ under flowing nitrogen. A heating mantle with built-in magnetic stirrer was used to heat the flask. The solution was stirred throughout the experiment with a stir bar, used in conjunction with the magnetic stirrer.

$0.3 \mathrm{~g}(\sim 1.5 \mathrm{mmol})$ of $\mathrm{Fe}\left(\mathrm{C}_{5} \mathrm{H}_{5}\right)\left(\mathrm{C}_{6} \mathrm{H}_{7}\right)$ was dissolved in a separately degassed mixture of $1.2 \mathrm{~mL}$ dichlorobenzene and $2.5 \mathrm{~mL}$ oleylamine. The bright red mixture was injected into the heated dichlorobenzene through the septum at the three-necked flask. The reaction mixture turned black within 2 minutes of injection, and was held at the reaction temperature for 2 hours under reflux.

\section{Purification}

The reaction was allowed to cool to room temperature naturally before the Fischer-Porter bottle or the three-necked flask was opened to air. For the reaction in a three-necked flask, the reaction mixture was transferred into a vial using pipette. The vial containing the reaction mixture was placed next to a magnet. Black precipitate was observed to be attracted to the magnet, and the solution was removed using a pipette without disturbing the precipitate to leave to a powder. About $10 \mathrm{~mL}$ of toluene was added to re-disperse the precipitate. If the precipitate did not instantly re-disperse, the mixture was sonicated for 1-2 minutes. The black precipitate was again attracted to the side of the vial using the magnet and the solution removed without disturbing the precipitate to leave to a powder, which was dried in air.

\section{Sample for TEM characterisation}

The magnetic precipitate collected was re-dispersed in toluene. The dispersion was sonicated to give a homogeneous suspension. A carbon-supported copper grid was held using self-closing tweezers and dipped into the suspension. This was carried out carefully such that the suspension was not in contact with the tweezers, as otherwise the magnetic nanoparticles would be attracted to the stainless steel tweezers and almost none would reside on the grid. The suspension was allowed to evaporate under ambient conditions and the TEM sample was ready. 


\subsection{References}

[1] D. L. Huber, Synthesis, properties, and applications of iron nanoparticles. Small 1, 482-501 (2005).

[2] C. M. Wang, D. R. Baer, L. E. Thomas, J. E. Amonette, S. Thevuthasan, J. Anthony and Y. Qiang, Microstructure of core-shell structured iron-iron oxide nanoparticles. Microsc. Microanal. 11, 1994-1995 (2005).

[3] C. M. Wang, D. R. Baer, L. E. Thomas, J. E. Amonette, J. Antony, Y. Qiang and G. Duscher, Void formation during early stages of passivation: Initial oxidation of iron nanoparticles at room temperature. J. Appl. Phys. 98, 094308 (2005).

[4] O. Bomati-Miguel, M. P. Morales, P. Tartaj, J. Ruiz-Cabello, P. Bonville, M. Santos, X. Zhao and S. Veintemillas-Verdaguer, Fe-based nanoparticulate metallic alloys as contrast agents for magnetic resonance imaging. Biomater. 26, 5695-5703 (2005).

[5] C. Xu and S. Sun, Monodisperse magnetic nanoparticles for biomedical applications. Polymer International 56, 821-826 (2007).

[6] Y.-W. Jun, J.-W. Seo and J. Cheon, Nanoscaling laws of magnetic nanoparticles and their applicabilities in biomedical sciences. Acc. Chem. Res. 41, 179-189 (2008).

[7] A. Tavakoli, M. Sohrabi and A. Kargari, A review of methods for synthesis of nanostructured metals with emphasis on iron compounds. Chem. Pap. - Chem. Zvesti 61, 151-170 (2007).

[8] J. Park, K. An, Y. Hwang, J.-G. Park, H.-J. Noh, J.-Y. Kim, J.-H. Park, N.-M. Hwang and T. Hyeon, Ultra-large-scale syntheses of monodisperse nanocrystals. Nat. Mater. 3, 891895 (2004).

[9] D. Kim, J. Park, K. An, N.-K. Yang, J.-G. Park and T. Hyeon, Synthesis of hollow iron nanoframes. J. Am. Chem. Soc. 129, 5812-5813 (2007).

[10] F. Dumestre, B. Chaudret, C. Amiens, P. Renaud and P. Fejes, Superlattices of iron nanocubes synthesised from $\mathrm{Fe}\left[\mathrm{N}\left(\mathrm{SiMe}_{3}\right)_{2}\right]_{2}$. Science 303, 821-823 (2004). 
[11] O. Margeat, F. Dumestre, C. Amiens, B. Chaudret, P. Lecante and M. Respaud, Synthesis of iron nanoparticles: Size effects, shape control and organisation. Progress in Solid State Chemistry 33, 71-79 (2005).

[12] K. S. Suslick, M. Fang and T. Hyeon, Sonochemical Synthesis of Iron Colloids. J. Am. Chem. Soc. 118, 11960-11961 (1996).

[13] J. P. Wilcoxon and P. P. Provencio, Use of surfactant micelles to control the structural phase of nanosize iron clusters. Journal of Physical Chemistry B 103, 9809-9812 (1999).

[14] E. E. Carpenter, Iron nanoparticles as potential magnetic carriers. J. Magn, Magn. Mater. 225, 17-20 (2001).

[15] J. Ren and R. D. Tilley Synthesis and characterisation of platinum and iron nanoparticles (Victoria University of Wellington, Wellington, New Zealand, 2007), Vol. MSc.

[16] S. Peng, C. Wang, J. Xie and S. Sun, Synthesis and stabilization of monodisperse Fe nanoparticles. J. Am. Chem. Soc. 128, 10676-10677 (2006).

[17] H. Liu, J. S. Owen and A. P. Alivisatos, Mechanistic study of precursor evolution in colloidal group II-VI semiconductor nanocrystal synthesis. J. Am. Chem. Soc. 129, 305312 (2007).

[18] R. M. de Silva, V. Palshin, K. M. N. de Silva, L. L. Henry and C. S. S. R. Kumar, A new role for surfactants in the formation of cobalt nanoparticles. J. Mater. Chem. 18, 738-747 (2008).

[19] A. Cabot, V. F. Puntes, E. Shevchenko, Y. Yin, L. Balcells, M. A. Marcus, S. M. Hughes and A. P. Alivisatos, Vacancy coalescence during oxidation of iron nanoparticles. J. Am. Chem. Soc. 129, 10358-10360 (2007).

[20] Y. Yin, R. M. Rioux, C. K. Erdonmez, S. M. Hughes, G. A. Somorjai and A. P. Alivisatos, Formation of hollow nanocrystals through the nanoscale Kirkendall effect. Science 304, 711-714 (2004).

[21] S. Laurent, D. Forge, M. Port, A. Roch, C. Robic, L. V. Elst and R. N. Muller, Magnetic iron oxide nanoparticles: synthesis, stabilization, vectorization, physicochemical characterizations, and biological applications. Chem. Rev. 108, 2064-2110 (2008). 
[22] C. H. Griffiths, M. P. O'Horo and T. W. Smith, The structure, magnetic characterization, and oxidation of colloidal iron dispersions. J. Appl. Phys. 50, 7108-7115 (1979).

[23] E. E. Carpenter, S. Calvin, R. M. Stroud and V. G. Harris, Passivated iron as core-shell nanoparticles. Chem. Mater. 15, 3245-3246 (2003).

[24] D. Farrell, S. A. Majetich and J. P. Wilcoxon, Preparation and characterization of monodisperse Fe nanoparticles. J. Phys. Chem. B 107, 11022-11030 (2003).

[25] D. Astruc, Organo-iron complexes of aromatic compounds. Applications in synthesis. Tetrahedron 39, 4027-4095 (1983). 


\section{Chapter 4}

\section{Synthesis and Characterisation of Iron \& Iron Carbide Nanoparticles}

\section{Chapter outline}

4.1. Introduction

4.2. Synthesis Results

4.2.1. Single-Step Heating

4.2.2. Two-Step Heating

4.2.3. Use of Excess Solvent

4.3. Discussion

4.3.1. Formation of Iron Carbide Nanoparticles

4.3.2. Nanoparticle Size and Morphology

4.4. Chapter Summary

4.5. Experimental

4.6. References

\subsection{Introduction}

This chapter describes the synthesis of iron and iron carbide nanoparticles at temperatures between $80-120^{\circ} \mathrm{C}$, and discusses how the different experimental conditions affect the nanoparticle composition, morphology, and size.

Work presented in this chapter is part of the research work on the synthesis and characterisation of iron-based nanoparticles. The initial aim of the research was to synthesise iron nanoparticles of larger than $10 \mathrm{~nm}$ in size, which would then oxidise on the surface leading to core/shell structures. During the investigation of the synthesis, iron carbide nanoparticles were identified among the nanoparticles collected, mostly from reactions at $120^{\circ} \mathrm{C}$ and below. The research was then taken further to include the synthesis 
of iron carbide nanoparticles and to investigate the various conditions that could possibly control the composition, morphology and size of the product.

This chapter focuses on syntheses at the lower temperature range $\left(80-120{ }^{\circ} \mathrm{C}\right)$ that produced both iron and iron carbide nanoparticles. Reactions at temperatures $130{ }^{\circ} \mathrm{C}$ and above produced mainly iron nanoparticles and are presented in Chapter 3 . The literature of iron nanoparticle synthesis is also reviewed in Chapter 3.

Bulk iron carbide is a metallic ferromagnet at room temperature and can be tuned to exhibit single domain magnetism on the nanometre scale.[1,2] Iron carbide nanoparticles are thus useful as the main substances in various magnetic applications such as ferrofluids and magnetic resonance imaging (MRI) contrast agents. ${ }^{[3,4]}$ Development of iron carbide nanoparticles for magnetic applications is relatively new as pure phase of iron carbide is difficult to achieve and usually requires high temperature and/or pressure. Most syntheses of iron carbide nanoparticles are of physical approach such as laser pyrolysis and sputtering. [3,5-7] Solution-phase synthesis of iron carbide nanoparticles via a chemical route has only been reported over the last few years; and only three methods have been reported to date. One of them is sonochemical method reported by Gedanken and co-workers, which produced nanocomposites of $\alpha$-Fe and $\mathrm{Fe}_{3}{ }_{3}$. $[8,9]$ Pure phase of $\mathrm{Fe}_{3} \mathrm{C}$ nanocrystals were reported by Nelson and Wagner, via an alkalide reduction method.[10] A challenge with both methods is the need to anneal the samples at very high temperatures, at $700{ }^{\circ} \mathrm{C}$ and $950{ }^{\circ} \mathrm{C}$, respectively, in order to obtain the carbide phase. A lower temperature synthesis was reported by $\mathrm{Yu}$ and Chow, in which iron-iron carbide composite nanoparticles were prepared by thermal decomposition of $\mathrm{Fe}(\mathrm{CO})_{5}$ under flowing $\mathrm{C}_{2} \mathrm{H}_{2}$ at $257{ }^{\circ} \mathrm{C}$, followed by annealing at $350^{\circ} \mathrm{C} .{ }^{[11]}$ It is thus highly interesting that iron carbide nanoparticles were observed in this study, with reactions undertaken at temperatures as low as $110^{\circ} \mathrm{C}$.

\subsection{Synthesis Results}

The synthesis is the same as that described in Chapter 3. For a typical experiment, the reaction solution was prepared by placing the iron precursor $\mathrm{Fe}\left(\mathrm{C}_{5} \mathrm{H}_{5}\right)\left(\mathrm{C}_{6} \mathrm{H}_{7}\right)$, oleylamine as the stabilising agent, and a solvent in a vial, which was in turn placed in a Fischer-Porter bottle. The Fischer-Porter bottle setup was filled with hydrogen to a desired pressure, and 
sealed. Reaction was carried out by placing the Fischer-Porter bottle into an oven heated at the reaction temperature.

Table 4-1. Summary table of synthesis.

\begin{tabular}{|c|c|c|c|c|c|c|c|c|}
\hline Expt & Solvent & $\begin{array}{l}\text { Conc. } \\
\text { (M) }\end{array}$ & $\mathrm{OLA}^{\mathrm{A}} / \mathbf{F e}$ & $\begin{array}{l}\text { Temp. } \\
\left({ }^{\circ} \mathrm{C}\right)\end{array}$ & Time & $\begin{array}{l}\mathrm{H}_{2} \text { press. } \\
\left(10^{2} \mathrm{kPa}\right)\end{array}$ & Nanoparticles $^{B}$ & $\begin{array}{l}\text { Avg. size } \\
\text { (nm) }\end{array}$ \\
\hline Fe-01 & Toluene & 0.05 & 3 & 110 & $4 d$ & 1 & Iron oxide & $6.5 \pm 1$ \\
\hline $\mathrm{Fe}-02$ & Toluene & 0.1 & 3 & 110 & $4 d$ & 1 & Fe- \& FeC-core, spherical & $18 \pm 2$ \\
\hline $\mathrm{Fe}-03$ & Toluene & 0.25 & 3 & 110 & $4 d$ & 1 & Fe- \& FeC-core, spherical & $22 \pm 3$ \\
\hline $\mathrm{Fe}-04$ & Toluene & 0.25 & 3 & 110 & $4 d$ & 3 & Fe- \& FeC-core, spherical & $23 \pm 5$ \\
\hline Fe-05 & Toluene & 0.5 & 3 & 110 & $3 d$ & 1 & $\begin{array}{l}\text { Fe-core, spherical, faceted } \\
\text { and branched }\end{array}$ & $25 \pm 5$ \\
\hline Fe-06 & Toluene & 1.0 & 3 & 110 & $2 d$ & 1 & $\begin{array}{l}\mathrm{Fe}-\& \mathrm{FeC} \text {-core, spherical } \\
\text { and sponge-like }\end{array}$ & $40 \pm 10$ \\
\hline Fe-07 & Toluene & 1.0 & 3 & 110 & $2 d$ & 3 & $\begin{array}{l}\text { Fe- } \& \text { FeC-core, spherical } \\
\text { and sponge-like }\end{array}$ & $40 \pm 10$ \\
\hline $\mathrm{Fe}-08$ & Mesitylene & 0.25 & 3 & 120 & $2 d$ & 1 & Fe- \& FeC-core, spherical & $22 \pm 3$ \\
\hline Fe-09 & Toluene & 0.25 & 1 & 110 & $4 d$ & 1 & Fe- \& FeC-core, spherical & $18 \pm 2$ \\
\hline $\mathrm{Fe}-10$ & Toluene & 0.25 & 0.2 & 110 & $4 d$ & 1 & $\begin{array}{l}\text { FeC-core, large } \\
\text { aggregations }\end{array}$ & $\geq 50$ \\
\hline $\mathrm{Fe}-11$ & Toluene & 0.25 & 6 & 110 & $3 d$ & 1 & $\begin{array}{l}\text { Fe- } \& \text { FeC-core, spherical } \\
\text { and sponge-like }\end{array}$ & $20-80$ \\
\hline $\mathrm{Fe}-12$ & Toluene & 1.0 & 1 & 110 & $2 d$ & 1 & $\begin{array}{l}\mathrm{Fe}(\& \mathrm{Fe}-\mathrm{C}) \text {, porous and } \\
\text { spherical }\end{array}$ & $50 \pm 10$ \\
\hline $\mathrm{Fe}-13$ & Toluene & 1.0 & 0.2 & 110 & $2 \mathrm{~d}$ & 1 & Hollow iron oxide & $7 \pm 2$ \\
\hline $\mathrm{Fe}-14$ & Toluene & 1.0 & 6 & 110 & $2 d$ & 1 & Fe-core, sponge-like & $65 \pm 15$ \\
\hline Fe-15 & - & - & 12 & 110 & $3 d$ & 1 & Fe-core, sponge-like & $65 \pm 15$ \\
\hline $\mathrm{Fe}-18$ & Toluene & 0.25 & 3 & 80,110 & $1 d, 3 d$ & 1 & Fe- \& FeC-core, spherical & $22 \pm 3$ \\
\hline $\mathrm{Fe}-17$ & Toluene & 0.5 & 3 & 80,110 & $1 d, 3 d$ & 1 & $\begin{array}{l}\text { Fe-core, spherical, } \\
\text { sponge-like and porous }\end{array}$ & $25 \pm 5$ \\
\hline $\mathrm{Fe}-16$ & Toluene & 1.0 & 3 & 80,110 & $1 d, 3 d$ & 1 & $\begin{array}{l}\text { FeC-core, spherical and } \\
\text { dimer }\end{array}$ & $18 \pm 6$ \\
\hline $\mathrm{Fe}-19$ & Mesitylene & 1.0 & 3 & 80,120 & $1 d, 3 d$ & 1 & Fe- \& FeC-core, spherical & $25 \pm 5$ \\
\hline
\end{tabular}

A OLA = oleylamine

В Fe-core = core/shell NPs with iron core; FeC-core = core/shell NPs with iron carbide core 
Table 4-1 lists the experimental conditions investigated, along with the composition, morphology and size of the nanoparticles obtained. A few reactions were carried out with excess solvent added to the Fischer-Porter bottle, but outside the vial containing the reaction mixture. These additional experiments are listed in Table 4-2. Characterisation results from selected experiments are presented in sub-sections following the summary tables.

Table 4-2. Summary table of synthesis involved the use of excess solvent.

\begin{tabular}{|c|c|c|c|c|c|c|c|}
\hline Expt $^{A}$ & $\begin{array}{c}\text { Conc. }^{B} \\
\text { (M) }\end{array}$ & $\begin{array}{c}\text { Excess } \\
\text { solvent }^{B} \\
(\mathrm{~mL})\end{array}$ & OLAC $/ \mathbf{F e}$ & $\begin{array}{c}\text { Temp. } \\
\left({ }^{\circ} \mathrm{C}\right)\end{array}$ & Time & $\begin{array}{l}\mathrm{H}_{2} \text { press. } \\
\left(10^{2} \mathrm{kPa}\right)\end{array}$ & Nanoparticles ${ }^{D}$ \\
\hline $\begin{array}{l}\mathrm{Fe}-20 \\
(\mathrm{Fe}-03)\end{array}$ & 0.25 & 5 & 3 & 110 & $4 d$ & 1 & Same as in Fe-03 \\
\hline $\begin{array}{c}\mathrm{Fe}-21 \\
(\mathrm{Fe}-06)\end{array}$ & 1.0 & 5 & 3 & 110 & $2 \mathrm{~d}$ & 1 & Same as in Fe-06 \\
\hline $\begin{array}{c}\mathrm{Fe}-22 \\
(\mathrm{Fe}-16)\end{array}$ & 0.25 & 5 & 3 & 80,110 & $1 d, 3 d$ & 1 & Same as in Fe-16 \\
\hline $\begin{array}{l}\mathrm{Fe}-23 \\
(\mathrm{Fe}-18)\end{array}$ & 1.0 & 5 & 3 & 80,110 & $1 d, 3 d$ & 1 & $\begin{array}{l}\text { Fe- \& FeC-core, spherical, } 25 \pm \\
5 \mathrm{~nm} \\
\text { FeC-core, dumbbell, } 50 \pm 20 \\
\mathrm{~nm}\end{array}$ \\
\hline Fe-24 & 1.0 & 20 & 3 & 80,110 & $1 d, 3 d$ & 1 & Polydisperse, 3-100 nm \\
\hline Fe-25E & 1.0 & 5 & 3 & 80,110 & $1 d, 3 d$ & 1 & $\begin{array}{l}\text { Iron oxide nanodisks, } 33 \pm 5 \\
\mathrm{~nm}\end{array}$ \\
\hline
\end{tabular}

A Parentheses indicate the experiments with the same reaction conditions but without the use of excess solvent

B Toluene was used as the solvent

C OLA = oleylamine

D Fe-core $=$ core $/$ shell NPs with iron core; FeC-core $=$ core $/$ shell NPs with iron carbide core

${ }^{\mathrm{E}} \mathrm{FeCl}_{3}$ was used as the precursor

\subsubsection{Single-Step Heating}

As shown in Table $4-1$, the synthesis was mainly investigated at $110^{\circ} \mathrm{C}$, by varying the precursor concentration and the amount of oleylamine used. The precursor concentration was varied from $0.05 \mathrm{M}$ to $0.2 \mathrm{M}$, in the presence of 3 equivalents oleylamine. At concentrations of 0.25 and $1.0 \mathrm{M}$, the use of oleylamine as the stabilising agent was investigated by varying the mole ratio of oleylamine to iron, from $0.2: 1$ to $6: 1$. The use of 
oleylamine as both stabiliser and solvent was also explored, where the oleylamine/iron ratio was 12 , which gave a concentration of $0.25 \mathrm{M}$ iron precursor in oleylamine. Experiments were carried out for 2 to 4 days, and at an initial atmosphere of $100 \mathrm{kPa} \mathrm{H}_{2}$ (except for Expt Fe-04 and Fe-07).

Apart from $110{ }^{\circ} \mathrm{C}$, reactions were also carried out at 80 and $120^{\circ} \mathrm{C}$. The nanoparticles obtained from the $120{ }^{\circ} \mathrm{C}$ reaction were observed to be the same as those collected at $110^{\circ} \mathrm{C}$ (which is described in Expt Fe-03 below). For the reaction at $80^{\circ} \mathrm{C}$, the reaction solution changed from bright red to dark red after 1 day, and turned darker over time, but no precipitate was observed after 4 days.

Different precursor concentrations were employed for reactions at $110^{\circ} \mathrm{C}$, the lowest being $0.05 \mathrm{M}$. For the low-concentration reaction, the resulting nanoparticles were observed to be the same as those obtained from the same precursor solution reacted at $130{ }^{\circ} \mathrm{C}$ (see Expt Fe_A, Section 3.2.1). The SAED pattern was indexed to iron oxide of the spinel phases. They were spherical or near-spherical in shape, with a nanoparticle size range of 5-8 nm.

\section{Expt Fe-03. Reaction of $0.25 \mathrm{M}$ iron precursor in toluene (middle concentration)}

In this experiment, a black precipitate that responded strongly to a magnet was collected after purification by magnetic separation. During purification, the precipitate in solution was observed to be attracted to the magnet almost instantly. In Expt Fe-01 (0.05 M), it took about 5 minutes for all precipitate to be attracted to the magnet.

Core/shell nanoparticles of $25 \pm 5 \mathrm{~nm}$ were obtained, as shown in the TEM image in Figure 4-1a. As shown in Figure 4-1b, EDS performed on a large ensemble of the nanoparticles including those in the TEM image showed presence of iron, oxygen, carbon, and copper. The possible compositions of the nanoparticles thus include iron, iron oxide, and iron carbide. The SAED pattern acquired from the same area was compared to that of iron/iron oxide core/shell nanoparticles described in Chapter 3 (Expt Fe_B, Section 3.2.1). Additional diffraction rings/spots to those of $\alpha$-Fe and spinel iron oxide are observed, as shown in Figure 4-1c. It was a challenge to index the diffraction pattern as the diffraction spots were very close and appear as bands. In Figure 4-1c, two bands can be observed, overlapping the diffraction rings/spots that correspond to $\{110\}$ and $\{211\}$ reflections of $\alpha$ - 
Fe. The bands, labelled as I and II, were attributed to spacings in the range 1.96-2.21 $\AA$ and 1.18-1.30 Å, respectively. These spacings could be matched more closely to the allowed peaks of bulk $\mathrm{Fe}_{3} \mathrm{C}$, than other form of iron carbide, as shown in Table 4-1. It is thus deduced that the iron carbide nanoparticles formed were mainly of the $\mathrm{Fe}_{3} \mathrm{C}$ phase.

As the reflections of $\mathrm{Fe}\{110\}$ and $\mathrm{Fe}\{211\}$ overlap with the spacings of $\mathrm{Fe}_{3} \mathrm{C}$, the only affirmative evidence of $\alpha$-Fe is from the diffraction of $\mathrm{Fe}\{200\}$. Based on this comparison, there is much larger number of diffraction spots corresponding to iron carbide than to iron, observation from the SAED pattern thus suggested a larger proportion of the core/shell nanoparticles to be of iron carbide core.

Core/shell nanoparticles of iron core comprised single-crystalline $\alpha$-Fe and polycrystalline iron oxide shell, as shown in Figure 4-2. The single crystal, cubic structure of the core was confirmed by the power spectrum of the HRTEM image, and lattice fringes measured were close to the corresponding $d$-spacings of $\alpha$-Fe.

In contrast, determination of the crystallinity, crystal structure, and orientation of iron carbide nanoparticles were less straightforward, mainly due to the complex crystal structure of iron carbide. An HRTEM image of a typical iron carbide/iron oxide core/shell nanoparticle is shown in Figure 4-3a, with a close-up at the core area of the nanoparticle shown in $\mathbf{b}$. The power spectrum of image b is shown in Figure 4-3c. It was not possible to recognise the crystal structure and orientation of the nanoparticle straight away from the power spectrum. Further analysis was carried out by measuring the lattice fringes on the HRTEM image, in which the measurement was calibrated with the lattice fringes of $\alpha$-Fe nanoparticles imaged at the same magnification. As shown in Figure 4-3b, lattice fringes were measured as 2.35, 2.06, and $1.96 \AA$, approximately matching the (210), (102), and (112) spacings of $\mathrm{Fe}_{3} \mathrm{C}$, respectively. Based on this information, together with the angles between the planes obtained from the power spectrum, the orientation of the nanoparticle

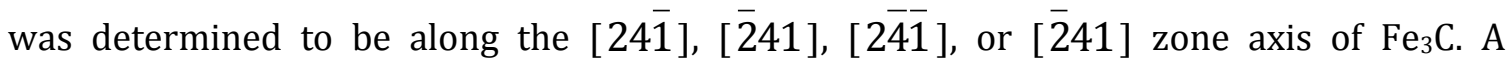
simulated [24 $\overline{1}$ ] projection of $\mathrm{Fe}_{3} \mathrm{C}$ is shown in Figure 4-3d, which can be well-matched by the power spectrum.

This experiment was repeated under a nitrogen atmosphere and the nanoparticles obtained were the same. 

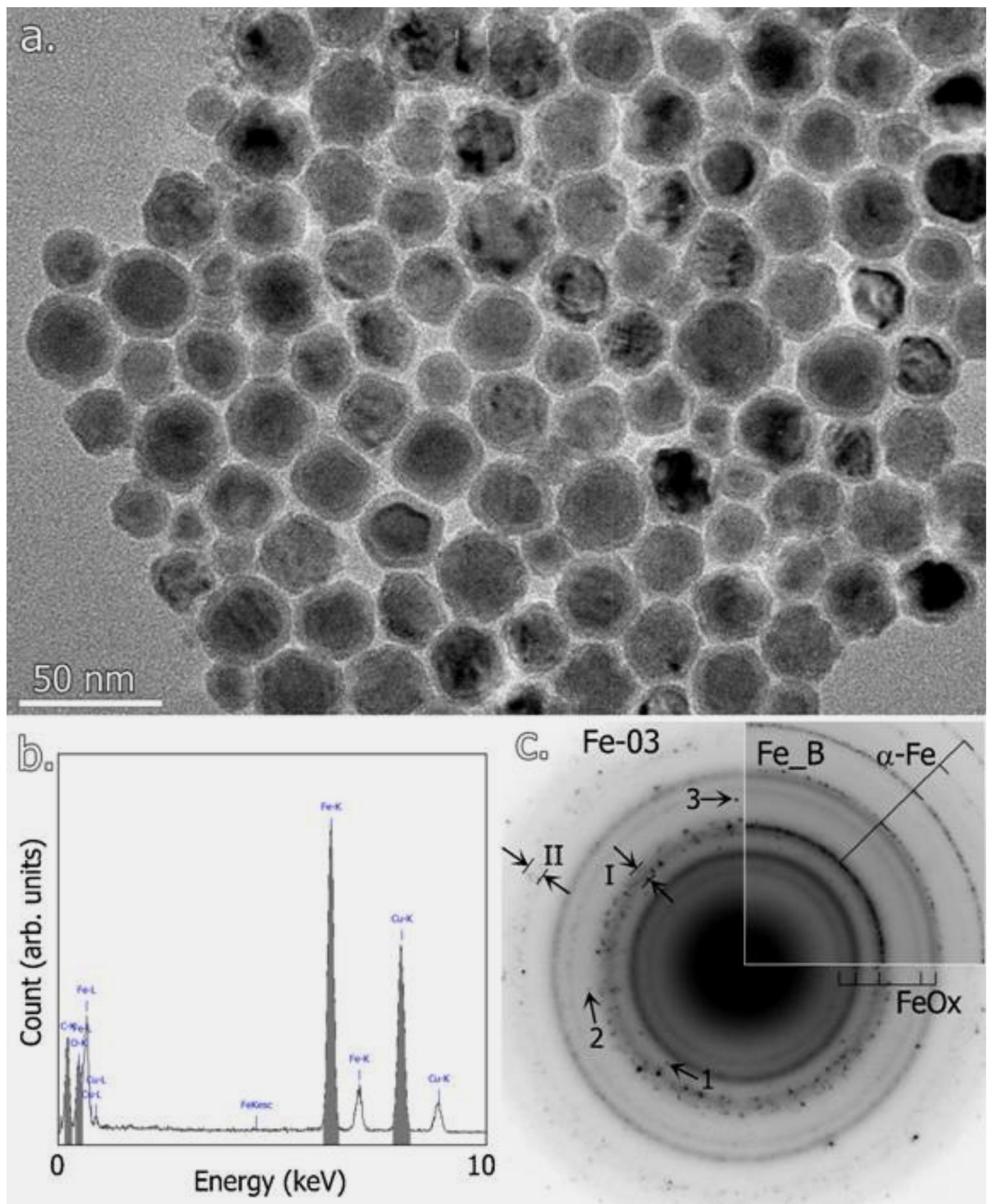

Figure 4-1. TEM and SAED results of middle-concentration reaction at $110^{\circ} \mathrm{C}(\mathrm{Fe}-03)$.

a. TEM image of core/shell nanoparticles of $20-30 \mathrm{~nm}$. b. EDS showing presence of iron (25 at\%) and oxygen (9 at\%) in the sample; carbon (47 at\%) was from the grid and the sample, where copper (19 at\%) was from the TEM grid. c. SAED pattern in comparison with that of Fe_B (which is shown in Chapter 3, Figure 3-2), showing additional diffraction spots to those of $\alpha$-Fe and spinel iron oxide (FeOx). 
Table 4-3. Index for SAED in Figure 4-1b.

\begin{tabular}{|c|c|c|c|c|c|c|c|c|c|c|}
\hline Index & Calc. $d$ & $\begin{array}{c}\text { Matched } \\
\text { d of } \\
\mathrm{Fe}_{3} \mathrm{C}\end{array}$ & I (\%) & h k l & $\begin{array}{c}\text { Matched } \\
d \text { of } \\
\mathrm{Fe}_{7} \mathrm{C}_{3}\end{array}$ & I (\%) & h k l & $\begin{array}{c}\text { Matched } \\
d \text { of } \\
\mathrm{Fe}_{5} \mathrm{C}_{2}\end{array}$ & I (\%) & h k l \\
\hline \multirow[t]{2}{*}{1} & \multirow[t]{2}{*}{2.36} & 2.388 & 43 & 121 & & & & & & \\
\hline & & 2.381 & 41 & 210 & & & & & & \\
\hline \multirow[t]{6}{*}{ I } & \multirow{6}{*}{$\begin{array}{c}2.21 \\
\mid \\
1.96\end{array}$} & 2.263 & 22 & 002 & 2.253 & 29 & 210 & 2.208 & 38 & 112 \\
\hline & & 2.218 & 22 & 201 & 2.121 & 43 & 102 & 2.083 & 100 & 021 \\
\hline & & 2.107 & 57 & 211 & 2.018 & 100 & 211 & 2.053 & 100 & 510 \\
\hline & & 2.068 & 67 & 102 & & & & 2.013 & 33 & 312 \\
\hline & & 2.031 & 56 & 220 & & & & 1.925 & 27 & 221 \\
\hline & & 1.977 & 100 & 031 & & & & & & \\
\hline \multirow[t]{3}{*}{2} & \multirow[t]{3}{*}{1.83} & 1.879 & 53 & 112 & 1.820 & 26 & 301 & & & \\
\hline & & 1.872 & 32 & 131 & 1.806 & 20 & 202 & & & \\
\hline & & 1.853 & 43 & 221 & & & & & & \\
\hline \multirow[t]{2}{*}{3} & \multirow[t]{2}{*}{1.73} & 1.763 & 19 & 122 & & & & 1.774 & 11 & 402 \\
\hline & & & & & & & & 1.733 & 11 & 421 \\
\hline \multirow[t]{5}{*}{ II } & \multirow{5}{*}{$\begin{array}{c}1.30 \\
\mid \\
1.18\end{array}$} & 1.329 & 17 & 123 & 1.204 & 11 & 303 & 1.218 & 18 & 114 \\
\hline & & 1.225 & 14 & 403 & 1.17125 & 10 & 322 & & & \\
\hline & & 1.219 & 16 & 133 & & & & & & \\
\hline & & 1.162 & 20 & 332 & & & & & & \\
\hline & & 1.152 & 15 & 251 & & & & & & \\
\hline
\end{tabular}




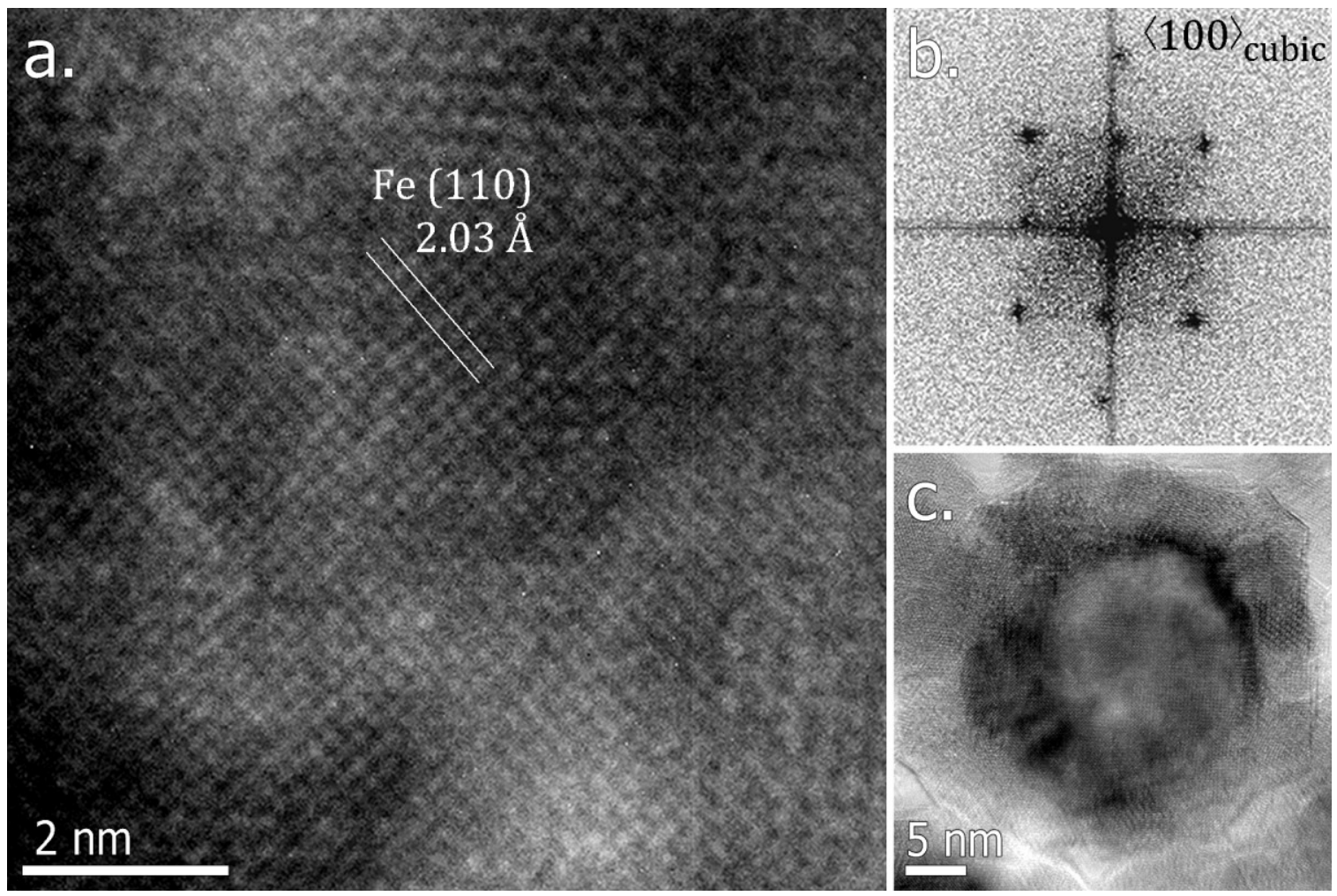

Figure 4-2. A typical iron/iron oxide core/shell nanoparticle obtained from the middle-concentration reaction at $110^{\circ} \mathrm{C}(\mathrm{Fe}-03)$.

a. HRTEM image of the core of a core/shell nanoparticle. b. Power spectrum of the HRTEM image, which can be matched to a $\langle 100\rangle$ projection of the cubic structure. c. TEM image of the whole nanoparticle. 

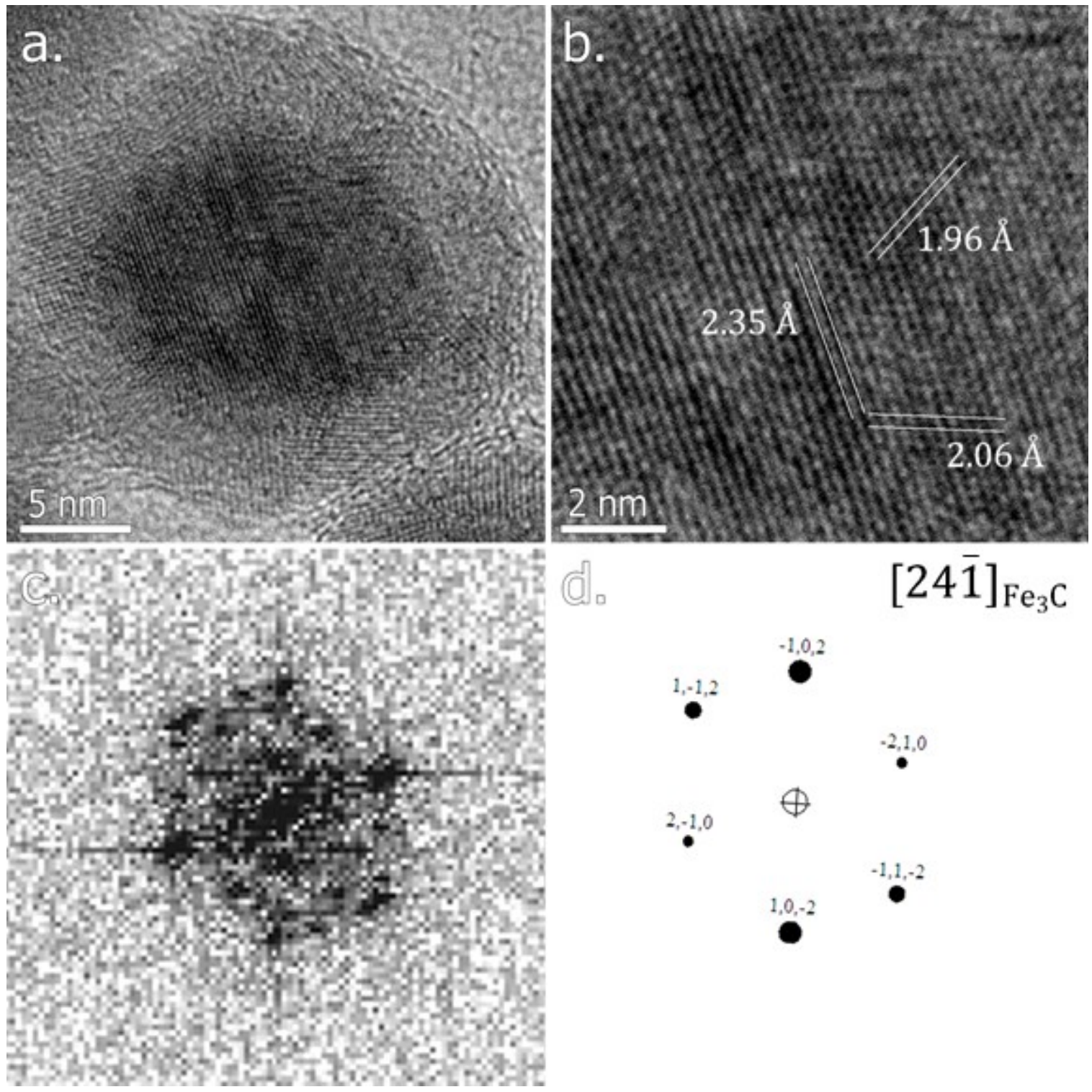

Figure 4-3. A typical iron carbide/iron oxide core/shell nanoparticle from the 0.25-M reaction at $110{ }^{\circ} \mathrm{C}(\mathrm{Fe}-03)$.

a. HRTEM image of a typical iron carbide/iron oxide core/shell nanoparticle, with a close-up of the image at the core area shown in image $\mathbf{b}$; lattice fringes were measured based on the lattice fringes of $\alpha-\mathrm{Fe}$ at the same magnification. c. Power spectrum of image $\mathrm{b}$, which can be matched to the [241] projection of $\mathrm{Fe}_{3} \mathrm{C}$. d. Simulated [241] projection of the orthorhombic $\mathrm{Fe}_{3} \mathrm{C}$. 


\section{Expt Fe-05. Reaction of middle-concentration solution added with 1 equivalent oleylamine}

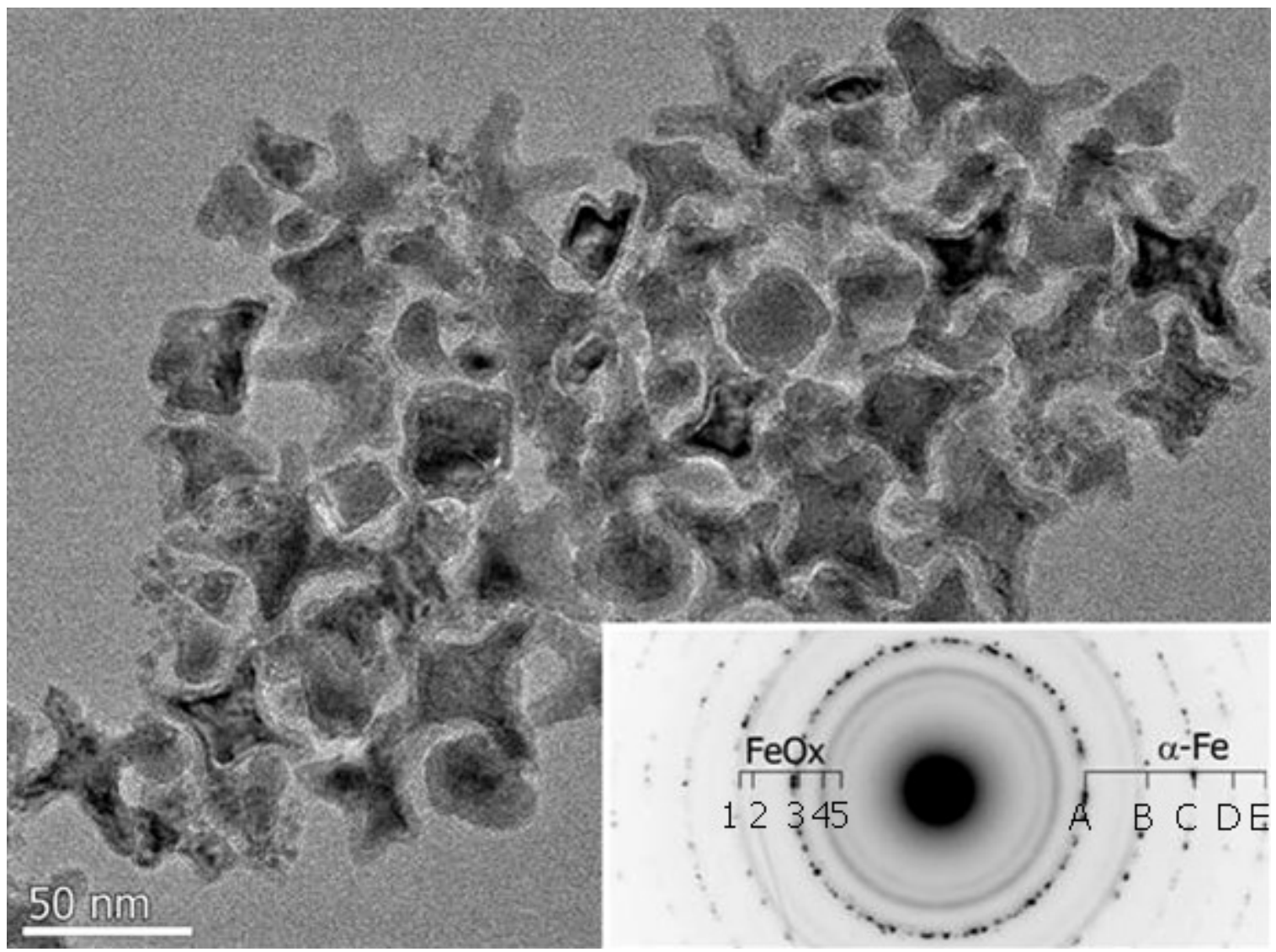

Figure 4-4. TEM and SAED results of middle-concentration reaction, with a 1:1 ratio of oleylamine to iron (Fe-05).

TEM image showing nanoparticles consisting of spherical, cube-like, and branched structures. SAED (inset) can be indexed to $\alpha$-Fe (diffraction rings A-E to $\{110\},\{200\},\{211\},\{220\}$, and $\{310\}$, respectively) and spinel iron oxide (FeOx; diffraction rings $1-5$ correspond to $\{440\},\{511\},\{400\}$, $\{311\}$, and $\{220\}$, respectively).

A mole ratio of 3 oleylamine to 1 iron precursor was used for most of the experiments. When the amount of oleylamine was reduced to a 1:1 ratio in a middle-concentration reaction, a black precipitate was collected after purification by magnetic separation. As shown in Figure 4-4a, core/shell nanoparticles of faceted and branched morphologies were observed. The branched nanoparticles were mainly tetrapods. The SAED shown in the inset can be indexed to $\alpha$-Fe and spinel iron oxides. The appearance of the strong $\alpha$-Fe diffraction spots and weaker iron oxide diffraction rings indicates a large $\alpha$-Fe crystallite, and small and/or polycrystalline structure of the iron oxide. Based on observations in the TEM, the core/shell nanoparticles comprised single crystalline $\alpha$-Fe core and polycrystalline iron oxide shell. Figure 4-5a shows the TEM image of a tetrapod core/shell nanoparticle, of 
which the HRTEM image of the core area is shown in Figure 4-5b. HRTEM and FFT analyses showed that the tetrapod core was an $\alpha$-Fe being projected along a $\langle 100\rangle$ zone-axis. The faceted nanoparticles observed in the sample were observed to terminate with $\{100\}$ facets.

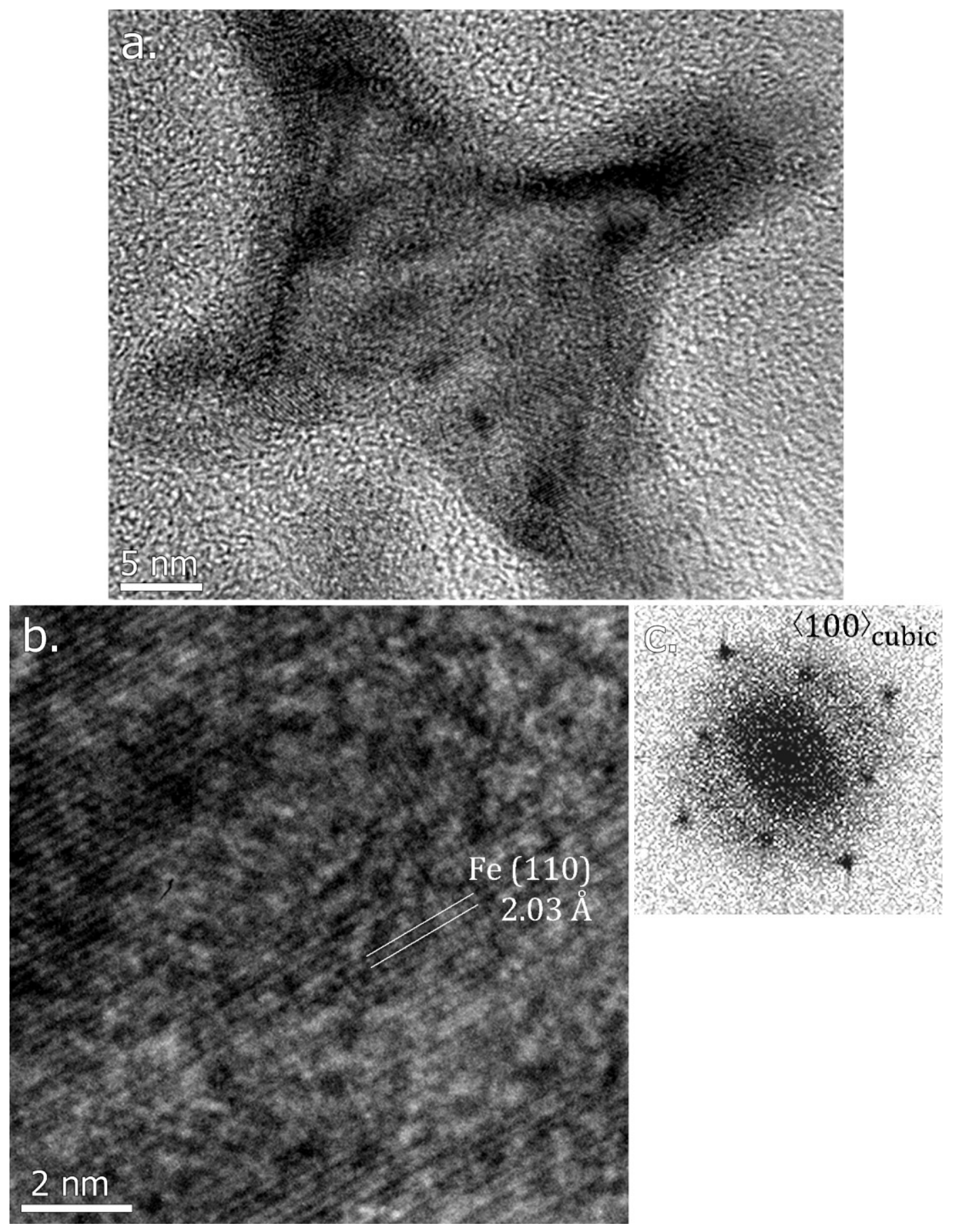

\section{Figure 4-5. A typical core/shell nanoparticle of tetrapod structure (Fe-05).}

a. TEM image of a typical tetrapod nanoparticle. b. HRTEM image of the core of the nanoparticle. c. Power spectrum of the HRTEM image which can be matched to a $\langle 100\rangle$ projection of cubic structure. The nanoparticle was projected along a $\langle 100\rangle$ zone-axis of bcc iron, with the four arms growing along the $\langle 110\rangle$ directions. 


\section{Expt Fe-06. Reaction of 1.0 M iron precursor in toluene (high concentration)}

In this experiment, the precursor concentration was increased 4 times to $1.0 \mathrm{M}$, and a magnetic precipitate was collected with similar observations to those observed for the middle-concentration reaction. The TEM image in Figure 4-6a shows that the sample consisted of aggregates of $\sim 5-\mathrm{nm}$ nanoparticles and core/shell nanoparticles of two different sizes: (i) 20-25 nm, and (ii) 50-80 nm. The SAED pattern in Figure 4-6b shows presence of $\alpha$-Fe, spinel iron oxide, and iron carbide in the sample.

HRTEM analysis showed that the nanoparticles of size range (i) were the iron carbide/iron oxide core/shell nanoparticles, whereas those of size range (ii) were of iron/iron oxide core/shell structures. The latter are described as the sponge-like nanoparticles as the nanoparticle morphology resembles that of a sponge. The same morphology was also observed in nanoparticles collected from a bench-top synthesis, Expt Fe_M (see Chapter 3, Section 3.3).

Figure 4-7a shows the powder XRD pattern of the sample, in which some of the peaks can be indexed to $\alpha$-Fe and iron oxide of the spinel phases. Peaks of much smaller intensity were observed near the Fe(110) peak, and can be approximately indexed to iron carbide of the $\mathrm{Fe}_{3} \mathrm{C}$ phase.

The diffraction rings in the SAED pattern and the peaks in the XRD pattern that correspond to $\alpha$-Fe were much higher in intensity than other peaks. The non-iron peaks are also noticeably broader. Based on the above observations it is concluded that the $\alpha$-Fe crystallites present in the sample are much larger in amount and size, which agrees with observation in the TEM. 

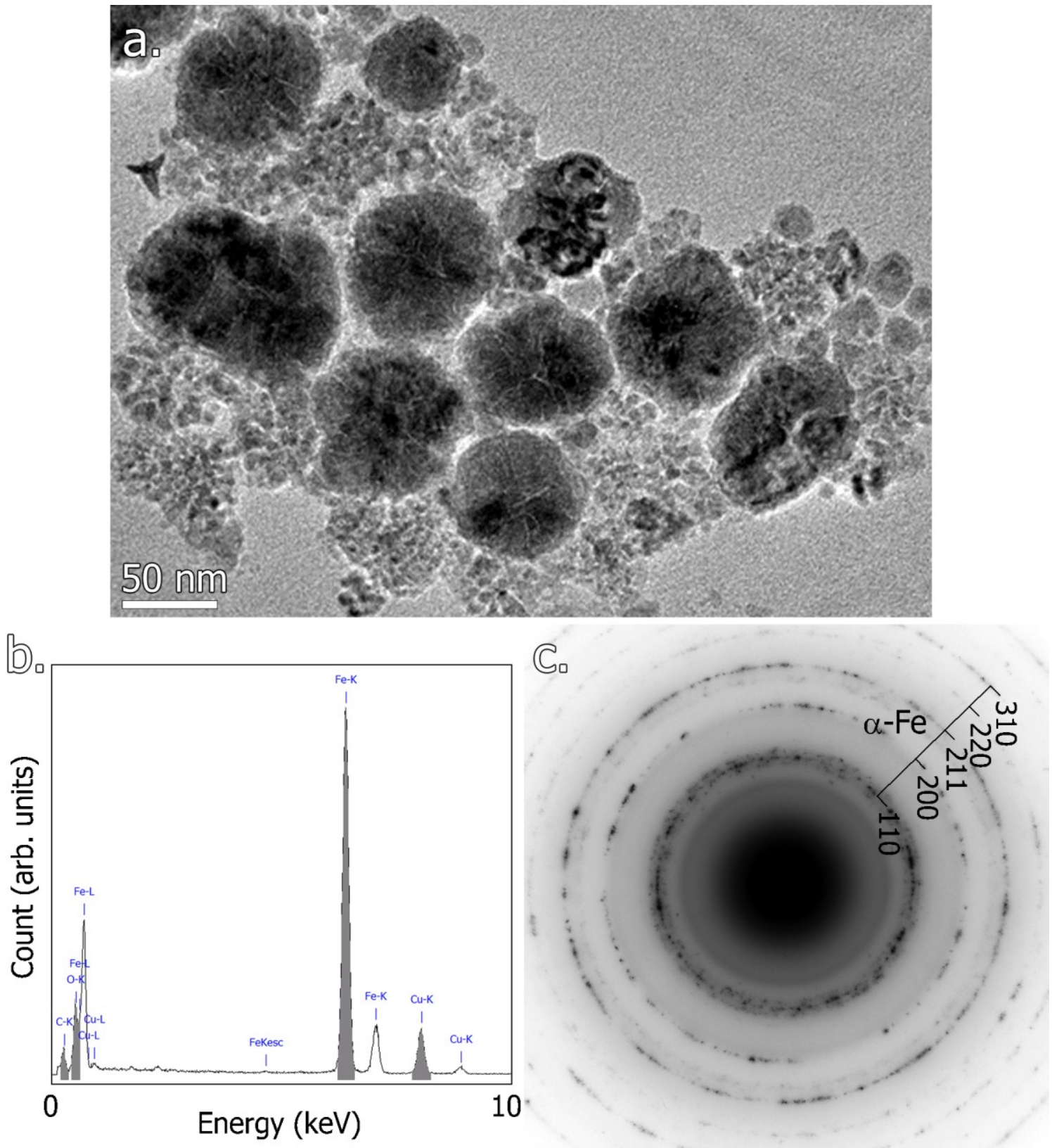

Figure 4-6. TEM and SAED results from high-concentration reaction at $110{ }^{\circ} \mathrm{C}(\mathrm{Fe}-06)$.

a. TEM image showing a group of sponge-like core/shell nanoparticles along with aggregated iron oxide nanoparticles. b. EDS showing presence of iron (52 at\%) and oxygen (20 at\%) in the sample; carbon (21 at\%) was from the grid and the sample, where copper (7 at\%) was from the TEM grid. c. Strong diffraction spots/rings can be indexed to $\alpha-\mathrm{Fe}$; a band of diffraction spots overlapping the $\mathrm{Fe}\{110\}$ diffraction ring indicates presence of crystalline iron carbide in the sample. 


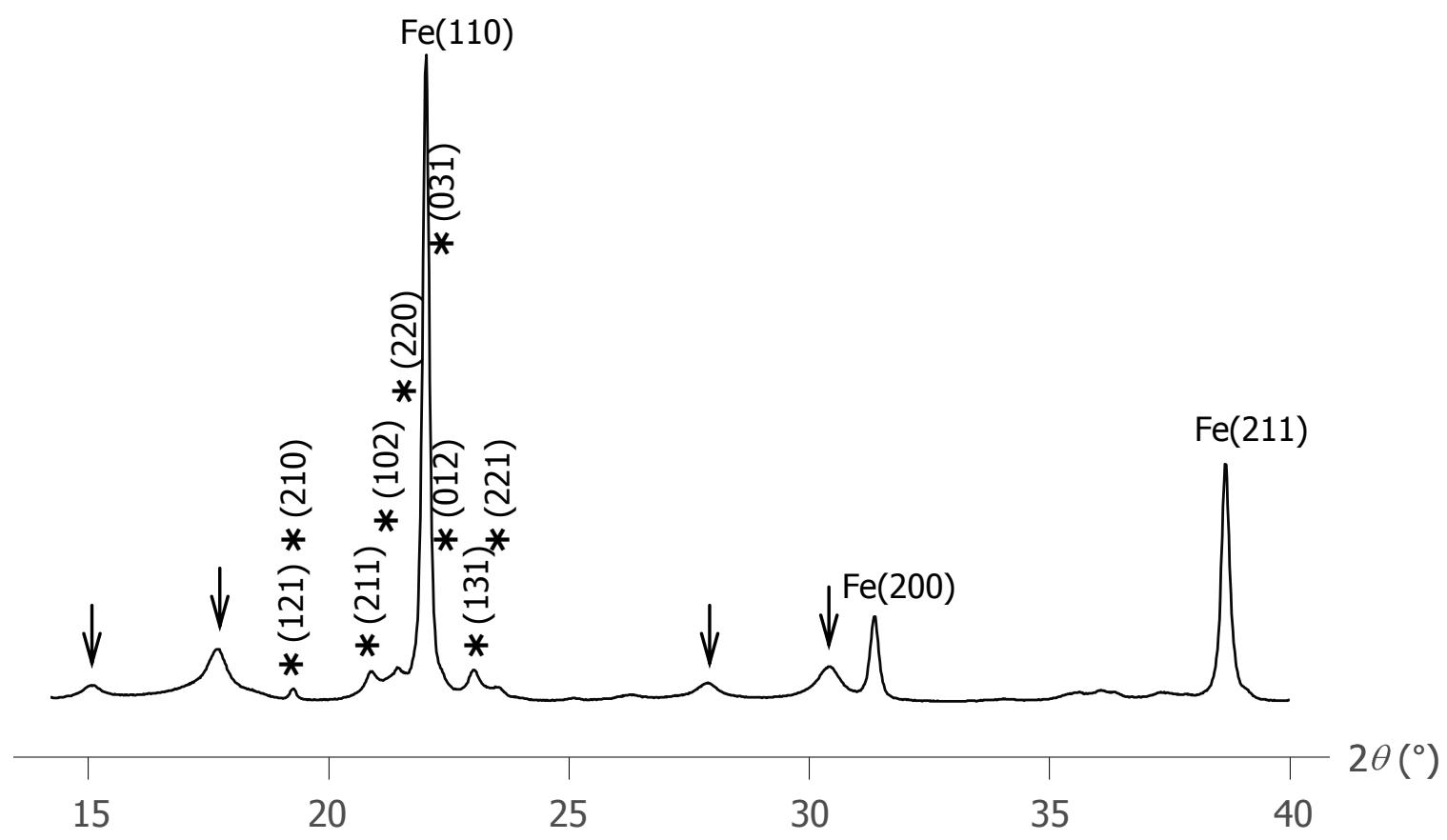

Figure 4-7. Powder XRD of nanoparticles from high-concentration reaction at $110{ }^{\circ} \mathrm{C}$ (Fe-06).

Peaks of much higher intensity correspond to diffraction of $\alpha$-Fe, the broader peaks indicated by arrows correspond to spinel iron oxide (220), (311), (511), and (440), from left to right respectively. Presence of iron carbide as the minor phase is shown by the small peaks, which are indexed to $\mathrm{Fe}_{3} \mathrm{C}(*)$.

The magnetic hysteresis loop of the sample measured at room temperature is shown in Figure 4-8a, with a close-up at the low field intervals shown in the inset. As can be observed in the low-field plot, the nanoparticles exhibited a ferromagnetic behaviour with a remanence magnetisation, $M_{R}$ of $\sim 31$ emu g-1, and a coercivity, $H_{C}$ of $\sim 450$ Oe. At the measured temperature, the sample did not saturate; the magnetisation, $M$ at an applied field, $H$ of $60 \mathrm{kOe}$ was measured to be 131.2 emu g- $^{-1}$. For the range $6 \leq H \leq 60 \mathrm{kOe}$, the magnetisation was observed to approach saturation according to the relation $M \propto H^{-1 / 2}$. From the plot of $M$ against $H^{-1 / 2}$, as shown in Figure 4-8b, the saturation magnetisation, $M_{S}$ was determined to be $\sim 137 \mathrm{emu} \mathrm{g}^{-1}$. 

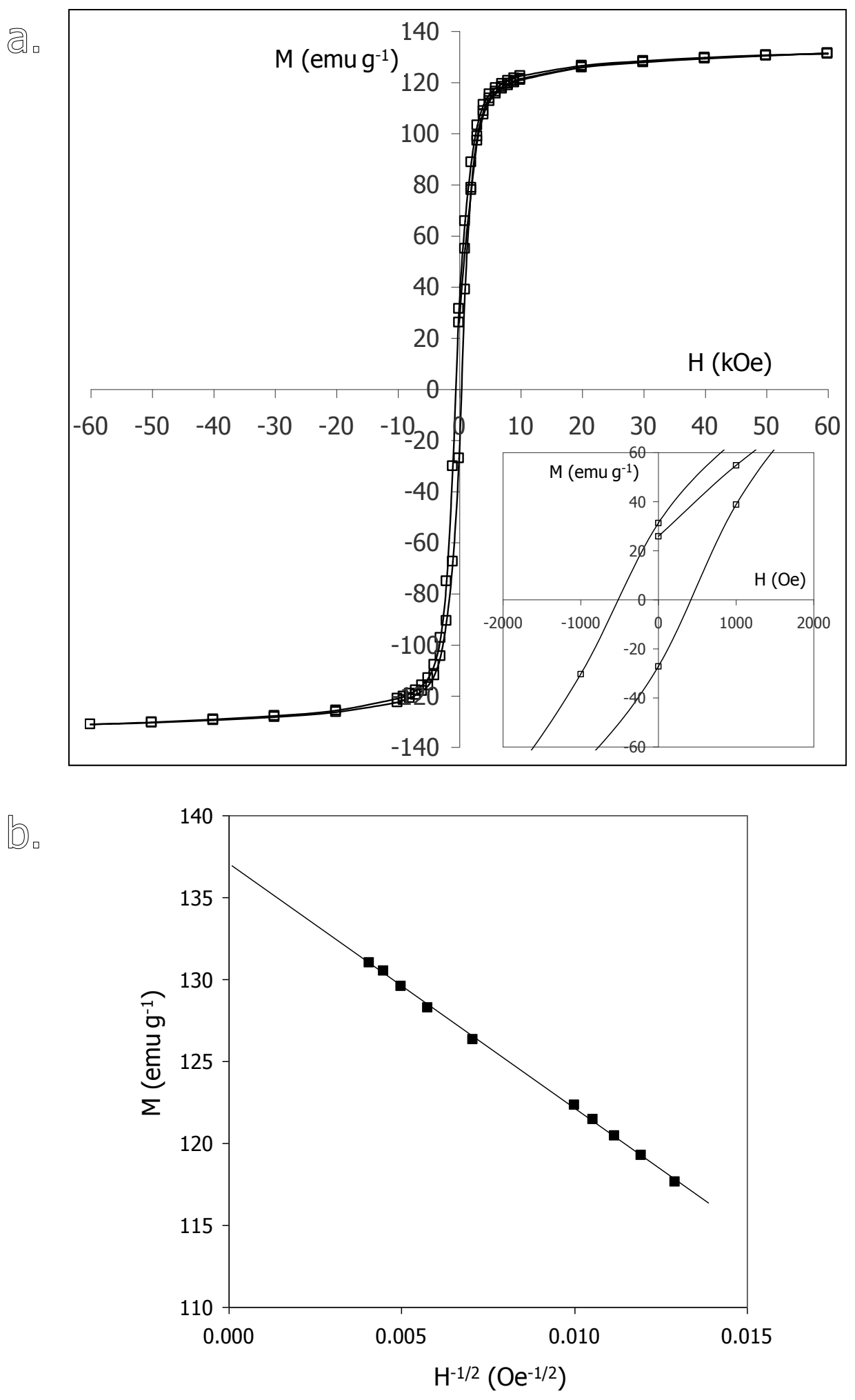

Figure 4-8. Magnetic properties of sample Fe-06 at $300 \mathrm{~K}$.

a. Plot of magnetisation, $\mathrm{M}$ against applied field, $\mathrm{H}$; inset shows plot at low field intervals.

b. The data was fitted with a linear function of $\mathrm{M}=-1490 \mathrm{H}^{-1 / 2}+137$ (shown by the solid line); $\mathrm{M}_{\mathrm{S}}$ was then determined to be $\sim 137 \mathrm{emu} \mathrm{g}^{-1}$ (intercept at $0 \mathrm{Oe}^{-1 / 2}$ ). 


\section{Expt Fe-12. Reaction of high-concentration solution added with 1 equivalent oleylamine}

A mole ratio of 3 oleylamine to 1 iron precursor was used for most of the experiments. When the amount of oleylamine was reduced to a 1:1 ratio in a high-concentration reaction, magnetic, dark grey to black precipitate was collected after purification by magnetic separation. As shown in Figure 4-9, the nanoparticles obtained were a mixture of core/shell and porous structures, with the latter being the major product and are described as porous nanoparticles. The SAED pattern, as shown in the inset, can be indexed to $\alpha$-Fe and spinel iron oxide.

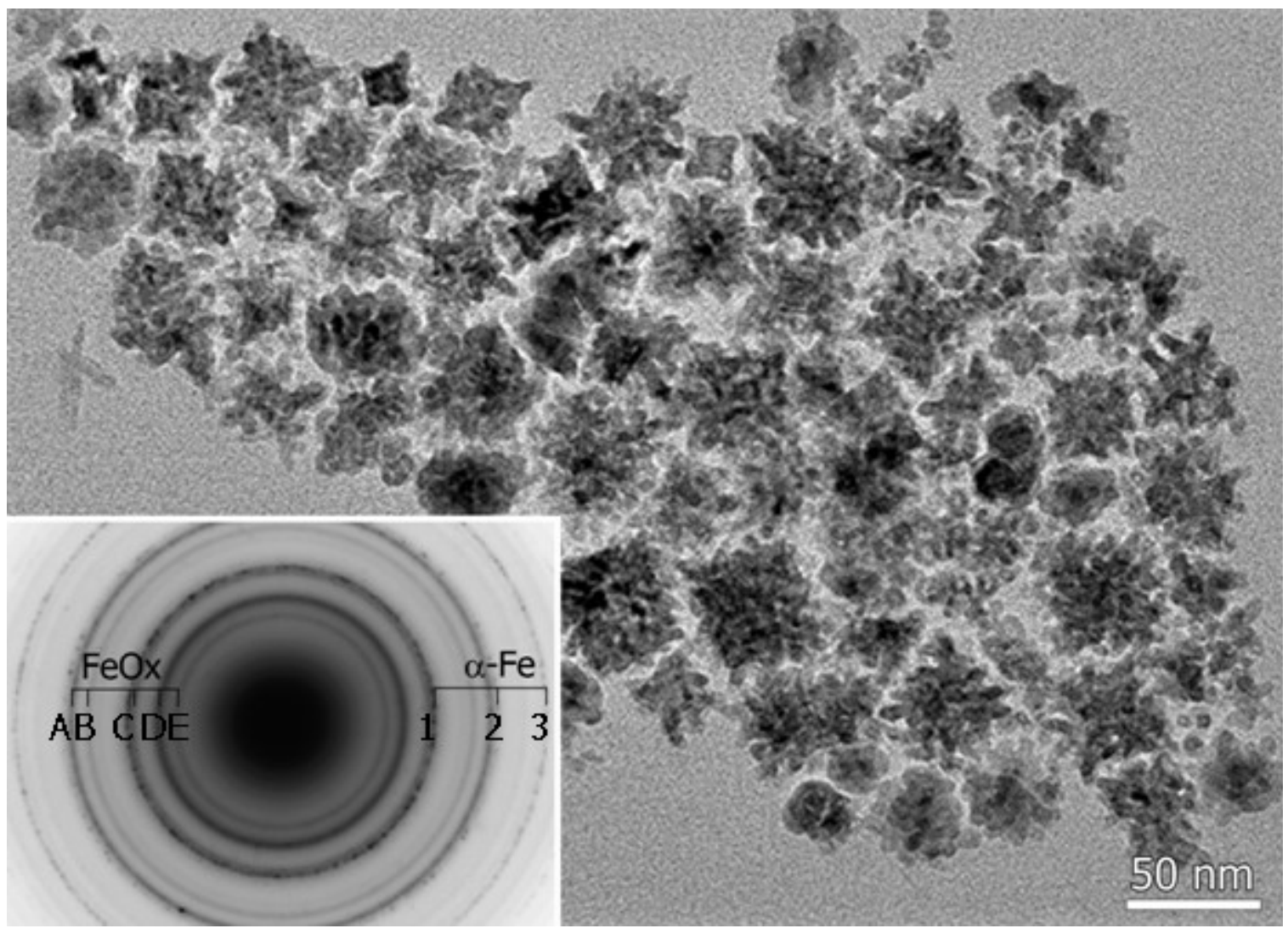

\section{Figure 4-9. TEM and SAED results of Expt Fe-12.}

TEM image showing core/shell nanoparticles mainly of porous structures. SAED (inset) can be indexed to $\alpha$-Fe (diffraction rings $1-3$ correspond to $\{110\},\{200\}$ and $\{211\}$, respectively) and spinel iron oxide (FeOx; diffraction rings A-E correspond to $\{440\},\{511\},\{400\},\{311\}$, and $\{220\}$, respectively).

Figure 4-10 shows the powder XRD pattern of the sample, in which peaks can be indexed to those of $\alpha$-Fe and spinel iron oxide. As the area under a diffraction peak is proportional to the relative abundance of the corresponding crystalline phase, the amount of $\alpha$-Fe in the 
sample is indicated to be similar to that of the spinel iron oxide. However, the $\alpha$-Fe peaks are relatively narrower, indicating the iron crystallites were larger in size compared to the iron oxide crystallites. The average $\alpha$-Fe crystallite size estimated from the fwhm of Fe(110) and $\mathrm{Fe}(211)$ was $19 \pm 2 \mathrm{~nm}$; whereas that of the iron oxide as estimated from the fwhm of the corresponding (311) and (511) peaks was $8.0 \pm 0.2 \mathrm{~nm}$. Close examination of the individual porous nanoparticles indicated that a typical porous nanoparticle comprised a single crystal $\alpha$-Fe core of porous structure.

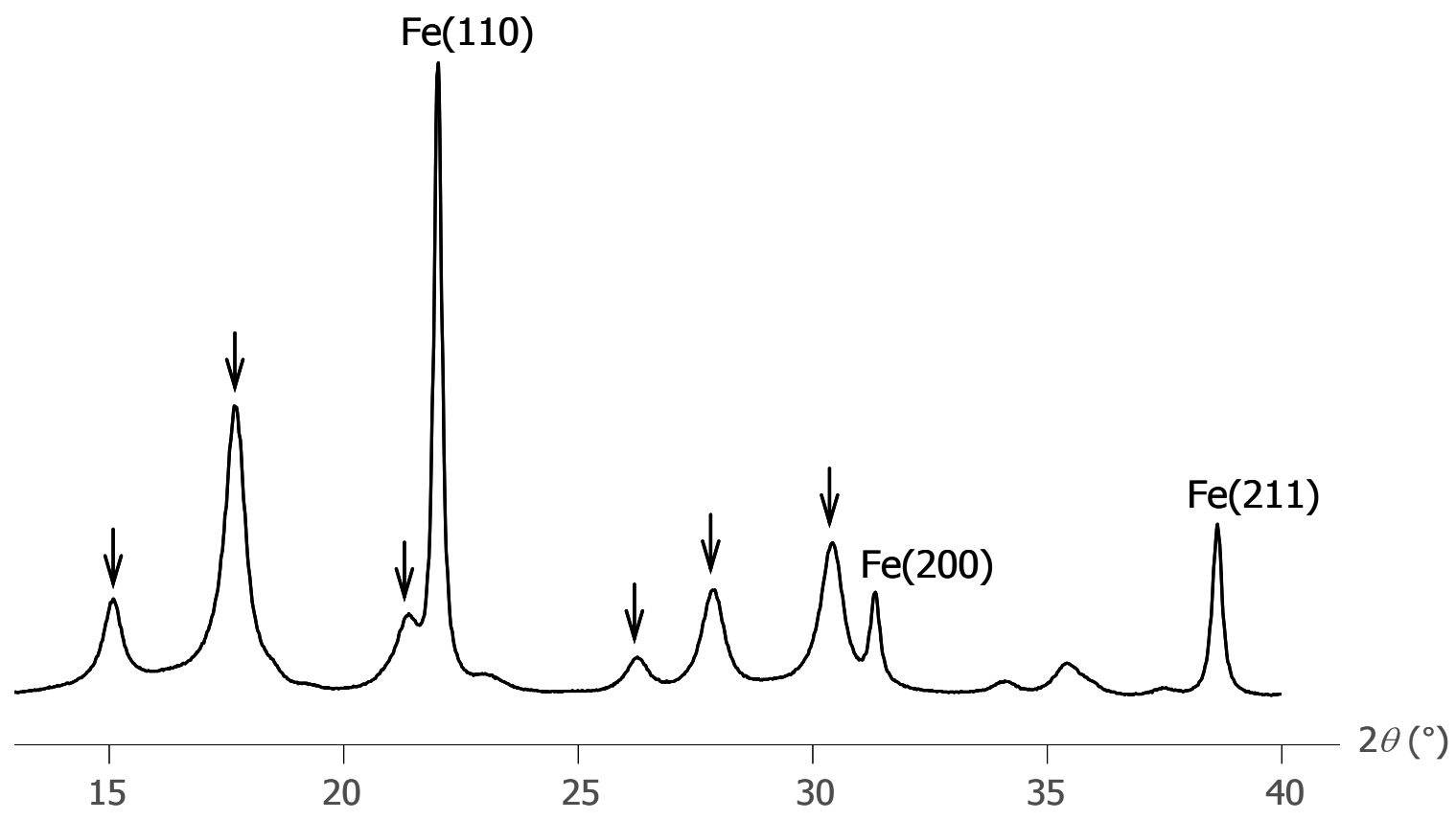

Figure 4-10. Powder XRD pattern of nanoparticles from Expt Fe-12.

Relatively narrower and higher intensity peaks correspond to the diffractions of $\alpha$-Fe, the broader peaks indicated by arrows correspond to spinel iron oxide (220), (311), (400), (422), (511), and (440), from left to right respectively.

\section{Expt Fe-13. Reaction of high-concentration solution added with 0.2 equivalents oleylamine}

When the amount of oleylamine was further reduced to 0.2 equivalents to iron precursor in a high-concentration reaction, magnetic, dark grey precipitate was obtained after purification by magnetic separation. 

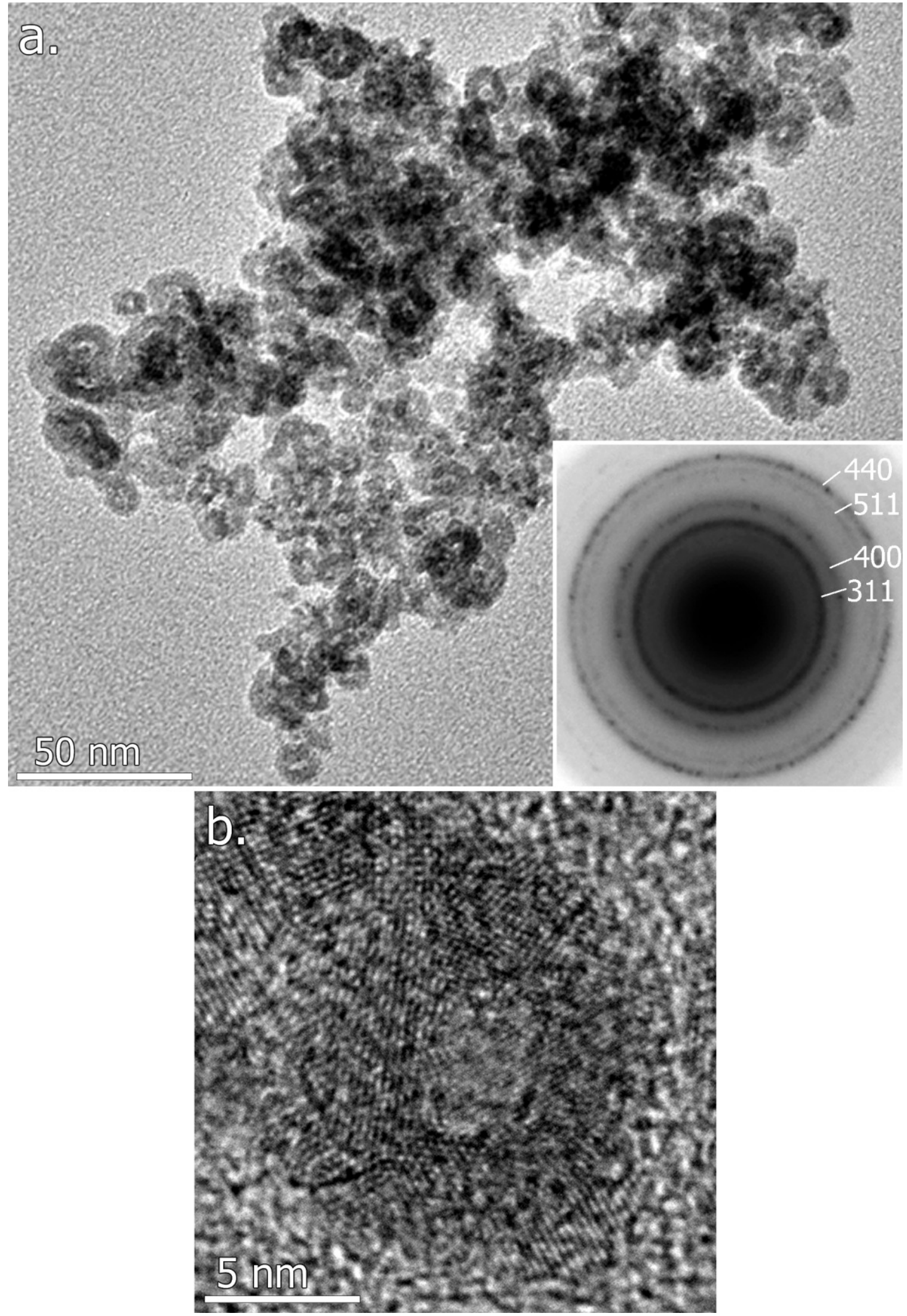

Figure 4-11. TEM and SAED results of high-precursor concentration reaction with 0.2 equivalents oleylamine ( $\mathrm{Fe}-13)$.

a. TEM image of the nanoparticles obtained, inset shows SAED pattern indexed to iron oxide of spinel phases. $\mathbf{b}$. HRTEM image of a typical hollow nanoparticle. 
As shown in Figure 4-11a, the nanoparticles adopted a hollow structure, of spherical or near-spherical in shape, averaging at $9 \pm 2 \mathrm{~nm}$. Nanoparticle aggregation could be observed. The SAED in the inset shows that these nanoparticles were of spinel iron oxide crystal structures. Figure 4-11b shows the HRTEM image of a typical hollow nanoparticle, which is of polycrystalline structure. As lattice fringes can be observed across the whole nanoparticle, the lighter contrast of the core area shows that the nanoparticle is of hollow and not of ring structure.

\subsubsection{Two-Step Heating}

Some of the reactions were heated in the oven over two different temperatures. Reactions involving a two-step heating process are often employed in solution-phase synthesis, in which reactants are heated at a certain temperature for a burst of nucleation event, and then at a lower temperature for a slower growth process. Attempts to react the precursor solution at a higher temperature $\left(130-160^{\circ} \mathrm{C}\right)$ followed by a lower temperature $\left(80-110^{\circ} \mathrm{C}\right)$ annealing had resulted in small nanoparticles that oxidised completely. Nevertheless, heating the high-concentration solution in the reverse order, i.e. from lower to higher temperature, managed to yield nanoparticles of interesting morphologies. The reaction at 80 then $120^{\circ} \mathrm{C}$ yielded the same result as that obtained from a reaction with single-heating temperature at 110 or $120^{\circ} \mathrm{C}$. Whereas the reaction at 80 then $110{ }^{\circ} \mathrm{C}$ yielded a mixture of spherical and dimer-like morphologies, which is described as follows.

\section{Expt Fe-16. Reaction of high-concentration reaction at 80 then $110{ }^{\circ} \mathrm{C}$}

In this experiment, the Fischer-Porter bottle was placed in the oven at $80^{\circ} \mathrm{C}$ for 1 day, after which the oven temperature was increased to $110{ }^{\circ} \mathrm{C}$ and the experiment was terminated after 3 days at $110{ }^{\circ} \mathrm{C}$. Figure 4-12a shows a TEM image of the nanoparticles obtained, which consisted of core/shell nanoparticles of spherical and dimer-like morphologies, with the core generally having a darker contrast. In the corresponding SAED pattern shown in Figure 4-12b, diffraction spots were indexed to iron or iron carbide, and diffraction rings to iron oxide. Observations in both the TEM image and SAED concluded that the nanoparticles 
comprised a core/shell structure, with an $\alpha$-Fe or $\mathrm{Fe}_{3} \mathrm{C}$ core and polycrystalline iron oxide shell. Figure 4-12c shows the TEM image of a typical dimer nanoparticle, which comprised two near-spherical nanoparticles attached together. As the oxide shell was mainly observed surrounding the whole dimer-like core, the dimer structure is believed to have formed in the synthesis, and not aggregate of two spherical nanoparticles after they were exposed to air.

The magnetic hysteresis loop of the sample measured at room temperature is shown in Figure 4-13a, with a close-up at the low field intervals shown in the inset. As shown in the low-field plot, the nanoparticles exhibited a ferromagnetic behaviour with a remanence magnetisation, $M_{\mathrm{R}}$ of $\sim 22$ emu g-1, and a coercivity, $H_{\mathrm{c}}$ of $\sim 480$ Oe. The sample did not saturate at the measured temperature; the magnetisation, $M$ at an applied field, $H$ of $60 \mathrm{kOe}$ was measured to be $94 \mathrm{emu} \mathrm{g}^{-1}$. The data could be fitted with the linear function of $M \propto H^{-1 / 2}$, and the saturation magnetisation, $M_{S}$ was determined to be $\sim 102.5 \mathrm{emu} \mathrm{g}^{-1}$, as shown in Figure 4-13b. 

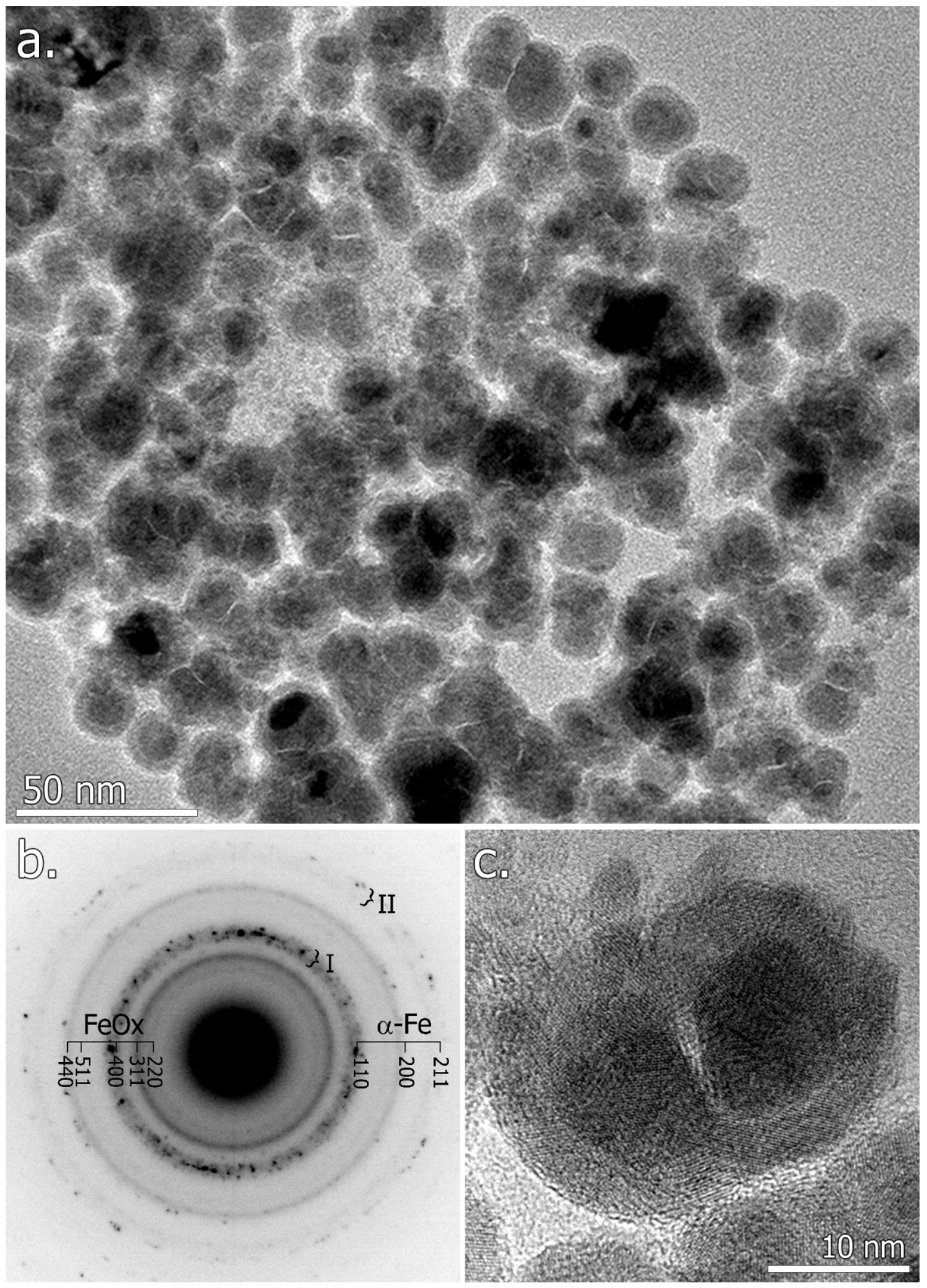

Figure 4-12. TEM and SAED results of middle-concentration solution reacted at 80 then $110{ }^{\circ} \mathrm{C}$ (Fe-16).

a. TEM image showing core/shell nanoparticles of spherical and dimer-like morphologies. $\mathbf{b}$. SAED indexed to $\alpha$-Fe and iron oxide of spinel phases (FeOx). Presence of crystalline iron carbide in the sample is indicated by the appearance of band-like arrangement of diffraction spots (I and II, see Figure 4-1 and Table 4-3). c. TEM image of a typical dimer nanoparticle. 

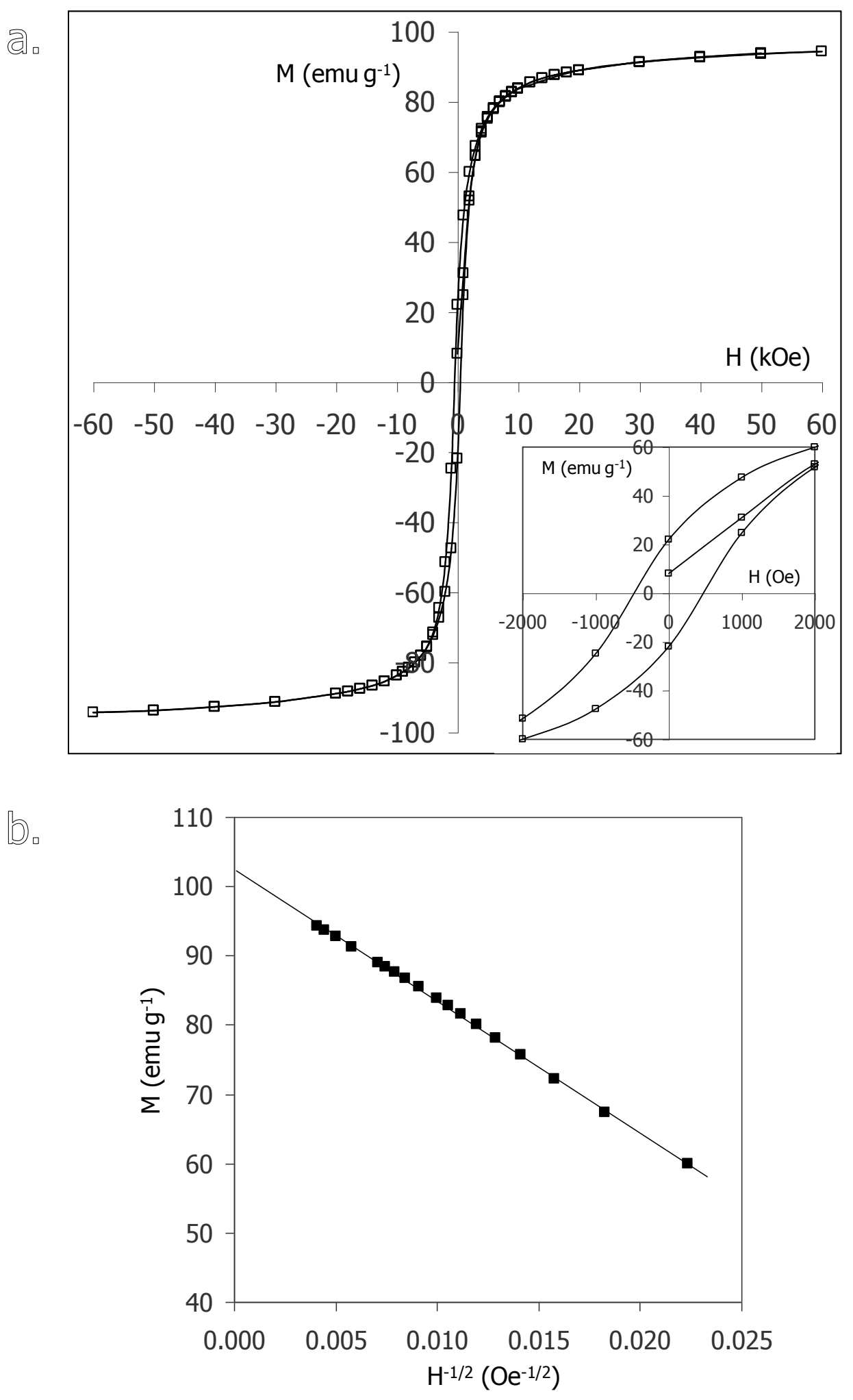

Figure 4-13. Magnetic properties of sample Fe-16 at $300 \mathrm{~K}$.

a. Plot of magnetisation, $\mathrm{M}$ against applied field, $\mathrm{H}$; inset shows plot at low field intervals.

b. The data was fitted with a linear function of $M=-1896 \mathrm{H}^{-1 / 2}+103$ (shown by the solid line); $\mathrm{M}_{\mathrm{S}}$ was thus determined to be $\sim 103 \mathrm{emu} \mathrm{g}^{-1}$ (intercept at $0 \mathrm{Oe}^{-1 / 2}$ ). 


\subsubsection{Use of Excess Solvent}

One particular observation in the synthesis in toluene, particularly the high-concentration reactions, was that the volume of solution was noticeably reduced at the end of the experiment. This was believed to be a result of evaporation of toluene due to high vapour pressure of toluene at the heating temperature of $110{ }^{\circ} \mathrm{C}$, and the change in volume is more obvious when the volume used is small in high-concentration reactions. Experiments Fe-03, Fe-06, Fe-16, and Fe-18 were repeated with an excess volume of solvent ( $5 \mathrm{~mL}$ ) added outside the vial. The hypothesis was to have the additional solvent contributing to the saturated solvent atmosphere when the reaction vessel was heated at $110{ }^{\circ} \mathrm{C}$, thus maintaining the volume of the reaction solution and hence the concentration in the vial. Apart from the repetition of Fe-16, labelled as Expt Fe-23, all other experiments yielded the same results as those from the respective experiments without the use of excess solvent (see Table 4-2). Nanoparticles of dumbbell-like structures were observed in Expt Fe-23, which is described below.

Due to the interesting dumbbell morphology observed in Expt Fe-23, the same reaction conditions were repeated with $\mathrm{Fe}\left(\mathrm{C}_{5} \mathrm{H}_{5}\right)_{2}, \mathrm{FeCl}_{3}, \mathrm{Fe}(\mathrm{acac})_{2}$, and $\mathrm{Fe}(\mathrm{acac})_{3}$ as the precursor source. $\mathrm{Fe}\left(\mathrm{C}_{5} \mathrm{H}_{5}\right)_{2}, \mathrm{Fe}(\mathrm{acac})_{2}$ and $\mathrm{Fe}(\mathrm{acac})_{3}$ did not react under the given reaction conditions. As for the reaction of $\mathrm{FeCl}_{3}$, nanoparticles of disk-like morphology were obtained and are described in Expt Fe-25 below. 


\section{Expt Fe-23. $1.0 \mathrm{M}+5 \mathrm{~mL}$ excess solvent, at 80 then $110{ }^{\circ} \mathrm{C}$}

As shown in Figure 4-14a, the nanoparticles obtained can be divided into three groups based on the nanoparticle morphology and size range: (i) nanoparticles of 5-8 $\mathrm{nm}$ that had aggregated; (ii) spherical or near-spherical core/shell nanoparticles of 20-40 nm; (iii) core/shell nanoparticles of dumbbell structure, which are described as the nanodumbbells. Figure 4-14b shows a high-magnification SEM image of the nanodumbbells, of which each is composed of two joined units that are of oblong shapes and are described as nanoellipsoids. The individual nanoellipsoids are typically 50-100 $\mathrm{nm}$ in length (the longest cross section of the nanoellipsoid) and 20-50 $\mathrm{nm}$ in width. As can be observed in both images $\mathbf{a}$ and $\mathbf{b}$ in Figure 4-14, the nanoellipsoids always joined side by side. It is also observed that the two nanoellipsoids constituting a nanodumbbell always come in almost the same size (both length and width), albeit the considerable size distribution of the nanodumbbells.

As shown in the TEM image (Figure 4-14a), the nanodumbbells tend to form into chains of 4-8 nanoparticles each, when the nanoparticle suspension dries on the TEM grid. It is also observed that the nanodumbbells align next to each in similar arrangements to how the nanoellipsoids are joined within a nanodumbbell. Figure 4-14c shows the SAED pattern with diffraction rings/spots corresponding to spacings of $\alpha$-Fe, iron carbide, and spinel iron oxide, in which most of the diffraction spots were attributed to iron carbide.

Figure 4-15a shows the TEM image of a typical nanodumbbell. The corresponding electron diffraction pattern in Figure 4-15b shows that the nanodumbbell is a single crystal. The SAED pattern can be indexed to a $\mathrm{Fe}_{3} \mathrm{C}$ being projected along the [001] zone axis; some of the (hk0) reflections are indicated in the figure. The nanoellipsoids are thus observed to have the long-axis along the [010] direction, which is the longest axis of the unit cell of orthorhombic $\mathrm{Fe}_{3} \mathrm{C}$ (unit cell details in Chapter 1, Section 1.6.). 

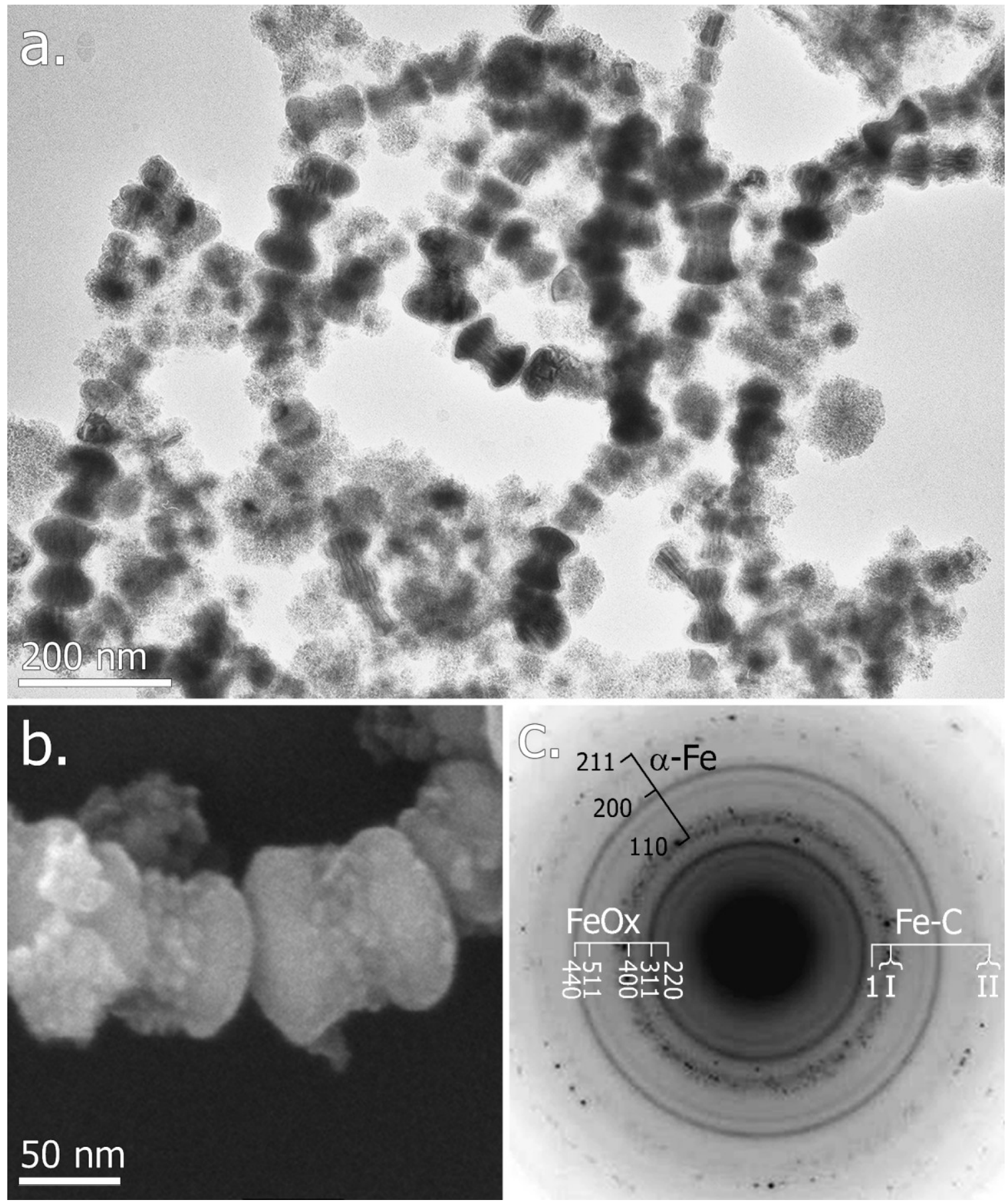

Figure 4-14. TEM and SAED results from high-concentration reaction, added with $5 \mathbf{~ m L}$ excess solvent outside reaction vial (Fe-23).

a. TEM image of the nanoparticles obtained. $\mathbf{b}$. High-magnification SEM image of the nanodumbbells. c. SAED pattern with diffraction ring indexed to $\alpha$-Fe and iron oxide of spinel phases (FeOx). Presence of crystalline iron carbide in the sample is confirmed by the appearance of band-like arrangement of diffraction spots (see Table 4-3). 


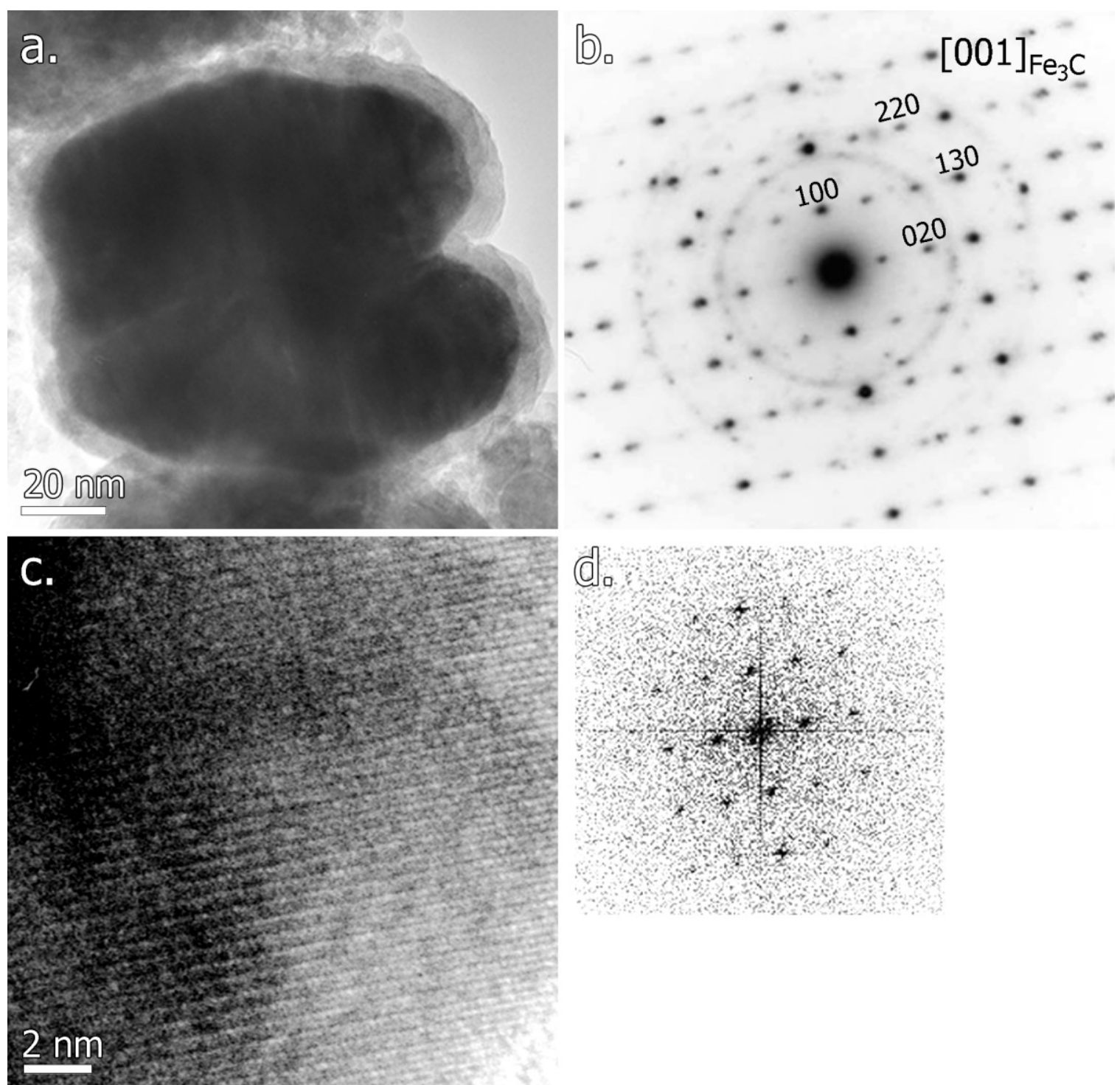

\section{Figure 4-15. HRTEM analysis of a nanodumbbell.}

a. TEM image of a typical nanodumbbell. b. SAED pattern of the nanodumbbell, which can be indexed to $\mathrm{Fe}_{3} \mathrm{C}$ being projected along the [001] zone axis. The background diffraction rings correspond to the spinel iron oxides, while other diffraction spots that do not match those of the $[001] \mathrm{Fe}_{3} \mathrm{C}$ orientation were from neighbouring nanodumbbells. c. HRTEM image of part of the core of the nanoparticle. d. Power spectrum of image $c$, which can be matched to the projection of $[001] \mathrm{Fe}_{3} \mathrm{C}$. 


\section{Expt Fe-25. $1.0 \mathrm{M} \mathrm{FeCl}_{3}+5 \mathrm{~mL}$ excess solvent, at 80 then $110{ }^{\circ} \mathrm{C}$}

In this experiment, the reaction solution was dark brown in colour at the end of the experiment, and turned lighter in brown during purification. Brown precipitate was collected via magnetic separation. The nanoparticles collected are shown in Figure 4-16. These nanoparticles displayed a faceted, disk-like morphology, with an average size of $35 \times 5 \mathrm{~nm}$ (diameter $\mathrm{x}$ thickness) and are described as nanodisks. The near-monodisperse nanodisks tend to assemble into long-range arrays when the nanoparticle suspension dries on a TEM grid. Figure 4-17a shows a TEM image of the nanoparticles stacking across the TEM grid, with the flat faces of the nanodisks assembled next to each other. In Figure 4-17b, the corresponding SAED pattern shows 6-fold symmetric crescents of diffraction spots corresponding to spinel iron oxide, indicating an almost identical crystallographic orientation of the nanodisks. In addition, reflections of $\alpha-\mathrm{Fe}_{2} \mathrm{O}_{3}$ (hematite) were also observed. It can be observed that the diffraction spots appear smeared, indicating a small shift or difference in the lattice spacings of the nanoparticles of the same phases. This shift could be a result of oxidation of the nanoparticles, leading a change of structure from the spinel phase to hematite. This thus explains the colour change during purification. The reflections closest to the central spot could be a result of the assembly of nanodisks of $\sim 5 \mathrm{~nm}$ in thickness.

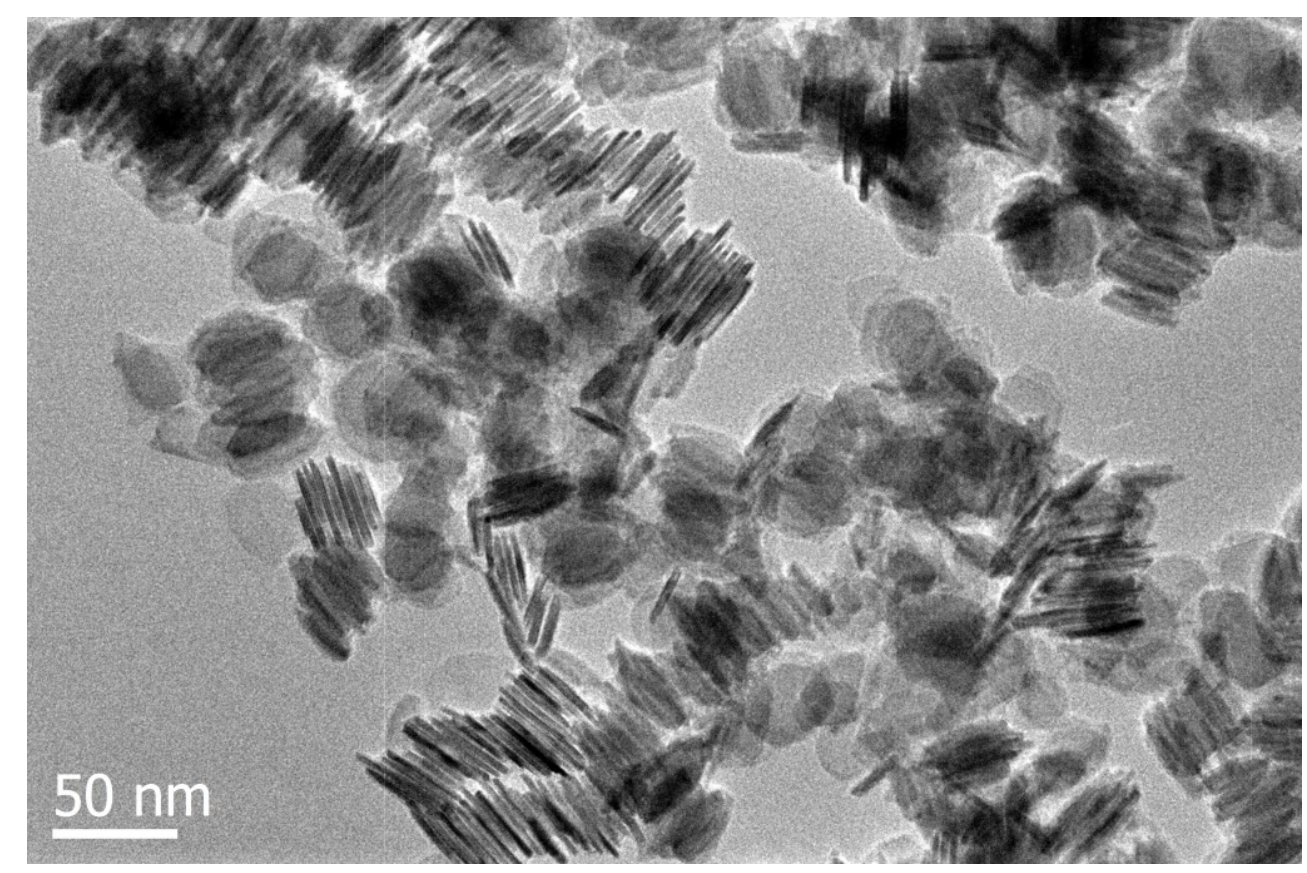

Figure 4-16. TEM image of nanoparticles obtained from a reaction of $\mathrm{FeCl}_{3}(\mathrm{Fe}-25)$. 


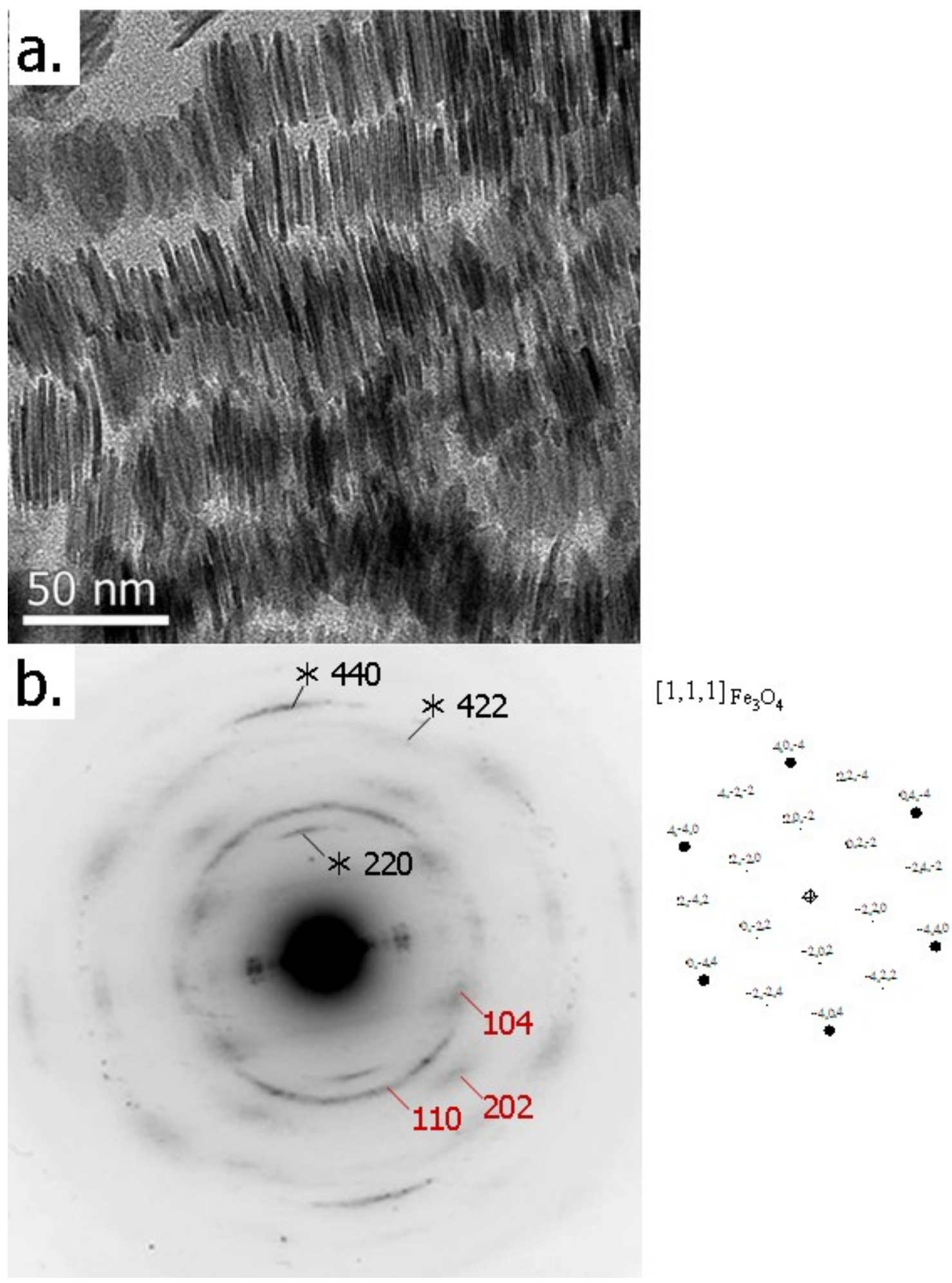

Figure 4-17. TEM and SAED results from reaction (Fe-25).

a. TEM image showing nanodisks assembled in long-range order. $\mathbf{b}$. The corresponding SAED showing specific orientation of the nanodisks; in which a 6-fold symmetry can be matched to the [111] projection of $\mathrm{Fe}_{3} \mathrm{O}_{4}(*)$, while others could be indexed to $\alpha-\mathrm{Fe}_{2} \mathrm{O}_{3}$ (in red). 


\subsection{Discussion}

\subsubsection{Formation of Iron Carbide Nanoparticles}

Results in this chapter showed that iron and iron carbide nanoparticles were synthesised through the decomposition of the iron precursor $\mathrm{Fe}\left(\mathrm{C}_{5} \mathrm{H}_{5}\right)\left(\mathrm{C}_{6} \mathrm{H}_{7}\right)$ employing the Fischer-Porter bottle technique, at $80-120^{\circ} \mathrm{C}$. For the formation of iron carbide nanocrystals, the carbon source is believed to have originated from the precursor. Different studies have demonstrated the formation of iron carbide as part of the process of growing carbon nanotubes that utilises iron as a catalyst.[12,13] It is thus believed that a similar catalytic process is involved in the formation of iron carbide in this study. The proposed mechanism is described as follows. The precursor first decomposes into free iron atoms, and gaseous and/or liquid hydrocarbons. The free iron atoms form into clusters, or nucleate and grow into nanoparticles of $\alpha$-Fe. The carbide formation starts with the (ironcatalysed) decomposition of hydrocarbons on the iron surfaces and diffusion of carbon species into the iron clusters or nanoparticles. The formation of iron carbide nanoparticles was observed to be largely influenced (promoted or hindered) by reaction temperature and precursor concentration.

Iron carbide nanoparticles were mainly observed in samples collected from experiments conducted at $80-120^{\circ} \mathrm{C}$; whereas higher temperature experiments, conducted at $130{ }^{\circ} \mathrm{C}$ and above, mainly produced $\alpha$-Fe nanoparticles (see Chapter 3). This observation indicates the effect of temperature on the availability of the source of carbon, which is believed to be the hydrocarbon part of the precursor. It has been shown that the mass spectral fragmentation of the precursor compound led to iron and hydrocarbons in cyclic forms of $\mathrm{C}_{5}$ and $\mathrm{C}_{6}{ }^{[14]}$ It is likely that the decomposition of the precursor occurs via similar paths. Thus apart from iron, other products of the decomposition could include benzene, cyclopentadiene, and possibly the derivatives of these molecules. As some of these compounds are highly volatile, they are less likely to remain in the solution at high temperatures for further reactions to form iron carbide.

At $110{ }^{\circ} \mathrm{C}$, a mixture of iron and iron carbide nanoparticles was obtained in most experiments, but high-concentration reactions $(1.0 \mathrm{M})$ were observed to yield higher proportion of iron nanoparticles. The volume of solvent is relatively small in a $1 \mathrm{M}$ solution, and hence the amount of hydrocarbons (as the carbon source for carbide formation) that 
could remain dissolved is limited. This is believed to have led to less iron carbide nanoparticles being formed.

\subsubsection{Nanoparticle Size and Morphology}

In the investigation of single-step heating, the nanoparticle morphology and size were observed to be influenced by both precursor concentration and the amount of oleylamine used, as summarised in Table 4-4.

The combined effect on nanoparticle size is observed to follow a similar trend as that observed for the synthesis at higher temperature (see Chapter 3); nanoparticle size and size distribution increase with the increase of precursor concentration and oleylamine to iron ratio.

The different morphologies observed include sponge-like, porous, hollow, faceted and branched nanoparticles. The sponge-like nanoparticles were favoured in reactions with large presence of oleylamine, whereas branched and porous (i.e. multiply-branched) structures were observed when the oleylamine to iron ratio was 1:1. This could be a result of the extent of effective interactions of the stabilising molecules with the precursor and/or reaction intermediate during the synthesis, as proposed and discussed in Chapter 3.

Nevertheless, a common condition shared by the reactions that yielded the non-spherical nanoparticles was a relatively high precursor concentration of 0.5-1 M. Reactions with high precursor concentration has been shown to produce branched and porous structures, the growth of which has been associated with fast growth rates.[15] The structures of branched, porous and sponge-like nanoparticles were rather similar suggesting a fast growth promoted by the high precursor concentration.

Additionally, the dimer nanoparticles and nanodumbbells were also obtained from high-concentration reactions. As the synthesis of these nanoparticles involved a two-step heating process ( 80 then $110^{\circ} \mathrm{C}$ ), the reaction and hence growth rates were expected to be influenced accordingly. Nevertheless, if growth was under a kinetically controlled regime, growth rates were expected to be higher than those of a lower concentration. As a result, growth of non-spherical structures is promoted. As for the mechanisms responsible for the formation of dimer- and dumbbell-like morphologies, further investigation is required. 
Table 4-4. Effect of precursor concentration on nanoparticle composition, morphology and size.

\begin{tabular}{|c|c|c|c|c|}
\hline $\begin{array}{l}\mathrm{OLA}^{*} / \mathrm{Fe} \\
\text { Conc. } \\
\text { (M) }\end{array}$ & 0.2 & 1 & 3 & 6 \\
\hline 0.05 & - & - & $7 \pm 2$, spherical & - \\
\hline 0.1 & - & - & $18 \pm 2$, spherical & - \\
\hline 0.25 & $\begin{array}{l}\geq 50, \\
\text { large aggregates }\end{array}$ & $18 \pm 2$, spherical & $22 \pm 3$, spherical & $\begin{array}{l}20-80, \text { spherical } \\
\& \text { sponge-like }\end{array}$ \\
\hline 0.5 & - & $\begin{array}{l}25 \pm 5, \text { faceted } \\
\& \text { branched }\end{array}$ & $25 \pm 5$, spherical & - \\
\hline 1.0 & $7 \pm 2$, hollow & $\begin{array}{l}40 \pm 10 \text {, spherical } \\
\& \text { porous }\end{array}$ & $\begin{array}{l}50 \pm 10 \text {, spherical } \\
\& \text { sponge-like }\end{array}$ & $\begin{array}{l}65 \pm 15 \\
\text { sponge-like }\end{array}$ \\
\hline
\end{tabular}

*OLA = oleylamine

\subsubsection{Magnetic Properties}

The magnetic properties of two samples, Fe-06 and Fe-16, were measured. Results showed that both samples did not saturate at the measured temperature. The same was also observed for sample Fe_B, as described in Chapter 3. Besides, the magnetisation, $M$ for all three samples were observed to approach saturation according to the relation $\mathrm{M} \propto H^{-1 / 2}$, where $H$ is the applied field. This unsaturation phenomenon has been observed for small nanoparticles of cobalt and iron, and iron/iron oxide core/shell structures.[16-19] A possible explanation for this to occur in the core/shell structures is the presence of a polycrystalline oxide shell, which is considered as a structure with multiple domains oriented in random fashion. ${ }^{[17,18]}$ It has been suggested that the oxide layers have frozen moments, which possess a resistance to the rotation by the applied field, hence resulting in unsaturation of the sample.[19]

The magnetic properties at room temperature are compared among the three samples, as shown in Table 4-5. The saturation magnetisation, $M_{S}$ is observed to be composition dependent. This is expected as $M_{S}$ of bulk iron is larger than that of iron carbide (218 and 140 emu g-1 $^{-1}$, respectively). $M_{S}$ is observed to be the highest for sample Fe-06, likely due to its larger average size of nanoparticle. The remanence magnetisation, $M_{R}$ is observed to share the same trend as $M_{S}$ does. However, this is not the case for the coercivity, $H_{C}$, where sample Fe-16 has the highest $H_{C}$ among the three. As high $H_{C}$ is caused by the presence of 
anisotropy, ${ }^{[20,21]}$ the high $H_{C}$ in this case is thus believed to be due to the presence of nanoparticles of dimer structures. The geometry of a dimer morphology is believed to have induced anisotropy, leading to a high $H_{C}$.

Table 4-5. Comparison of magnetic properties of different nanoparticles.

\begin{tabular}{|c|c|c|c|c|c|}
\hline Expt & Nanoparticles & $\begin{array}{l}\text { Avg. size } \\
\text { (nm) }\end{array}$ & $\begin{array}{c}M s \\
\left(\text { emu g-1) }^{-1}\right)\end{array}$ & $\begin{array}{c}H \mathrm{C} \\
(\mathrm{Oe})\end{array}$ & $\begin{array}{c}M_{\mathrm{R}} \\
\left(\mathrm{emug}^{-1}\right)\end{array}$ \\
\hline $\mathrm{Fe}_{-} \mathrm{B}$ & Fe-core, spherical & $14 \pm 2$ & 131 & 370 & 15 \\
\hline $\mathrm{Fe}-06$ & Fe- \& FeC-core, spherical and sponge-like & $40 \pm 10$ & 137 & 450 & 31 \\
\hline Fe-16 & FeC-core, spherical and dimer & $18 \pm 6$ & 103 & 480 & 22 \\
\hline
\end{tabular}

\subsection{Chapter Summary}

Highly crystalline $\alpha$-Fe and $\mathrm{Fe}_{3} \mathrm{C}$ nanoparticles were synthesised through the decomposition of the iron precursor using the Fischer-Porter bottle method, at temperatures in the range of $80-110^{\circ} \mathrm{C}$. The $\mathrm{Fe}_{3} \mathrm{C}$ phase was confirmed by HRTEM characterisation performed on individual nanoparticles.

The synthesis was investigated by employing different reaction conditions. Formation of $\mathrm{Fe}_{3} \mathrm{C}$ was observed to be mainly influenced by reaction temperature and precursor concentration. Nanoparticles were generally bigger than those obtained from reactions at higher temperatures (as described in Chapter 3). Nanoparticle size was observed to be governed by precursor concentration and the relative oleylamine (stabiliser) concentration. Non-spherical morphologies observed for core/shell nanoparticles in different samples include dimer, dumbbell, porous, faceted and tetrapod structures. These nanoparticles were mainly obtained from reactions with high precursor concentration in the range of 0.5-1.0 M.

\subsection{Experimental}

The materials, synthesis and purification details were the same as those described in the Experimental section in Chapter 3, except that toluene was used for almost all of the experiments described in this chapter. 


\subsection{References}

[1] A. Tsuzuki, S. Sago, S.-I. Hirano and S. Naka, High temperature and pressure preparation and properties of iron carbides $\mathrm{Fe}_{7} \mathrm{C}_{3}$ and $\mathrm{Fe}_{3}$ C. J. Mater. Sci. 19, 25132518 (1984).

[2] T. J. Goodwin, S. H. Yoo, P. Matteazzi and J. R. Groza, Cementite-iron nanocompositie. Nanostruct. Mater. 8, 559-566 (1997).

[3] Nanocrystalline $\alpha$ - $\mathrm{Fe}, \mathrm{Fe}_{3} \mathrm{C}$, and $\mathrm{Fe}_{7} \mathrm{C}_{3}$ produced by $\mathrm{CO}_{2}$ laser pyrolysis. J. Mater. Res. 8, 1666-1674 (1993).

[4] X. Q. Zhao, Y. Liang, Z. Q. Hu and B. X. Liu, Oxidation characteristics and magnetic properties of iron carbide and iron ultrafine particles. J. Appl. Phys. 80, 5857-5860 (1996).

[5] C. A. Grimes, J. L. Horn, G. G. Bush, J. L. Allen and P. C. Eklund, Curie temperature enhancement in $\mathrm{Fe}_{3} \mathrm{C}$ nanoparticles made by laser pyrolysis. IEEE Trans. Magn. 33, 3736-3738 (1997).

[6] S. Tajima and S.-i. Hirano, Synthesis and properties of Fe3C film by RF magnetron sputtering. J. Mater. Sci. 28, 2715-2720 (1993).

[7] H. Yumoto, Y. Nagamine, J. Nagahama and M. Shimotomai, Corrosion and stability of cementite films prepared by electron shower. Vacuum 65, 527-531 (2002).

[8] S. I. Nikitenko, Y. Koltypin, O. Palchik, I. Felner, X. N. Xu and A. Gedanken, Synthesis of highly magnetic, air-stable iron-iron carbide nanocrystalline particles by using power ultrasound. Angew. Chem. Int. Ed. 40, 4447-4449 (2001).

[9] S. I. Nikitenko, Y. Koltypin, I. Felner, I. Yeshurun, A. I. Shames, J. Z. Jiang, V. Markovich, G. Gorodetsky and A. Gedanken, Tailoring the properties of $\mathrm{Fe}-\mathrm{Fe}_{3} \mathrm{C}$ nanocrystalline particles prepared by sonochemistry. J. Phys. Chem. B 108, 7620-7626 (2004).

[10] J. A. Nelson and M. J. Wagner, High surface area nanoparticulate transition metal carbides prepared by alkalide reduction. Chem. Mater. 14, 4460-4463 (2002). 
[11] S. Yu and G. M. Chow, Synthesis, structural, magnetic, and cytotoxic properties of iron oxide coated iron/iron-carbide nanocomposite particles. J. Appl. Phys. 98, 114306 (2005).

[12] C. Emmenegger, J. M. Bonard, P. Mauron, P. Sudan, A. Lepora, B. Grobety, A. Zuttel and L. Schlapbach, Synthesis of carbon nanotubes over Fe catalyst on aluminium and suggested growth mechanism. Carbon 41, 539-547 (2003).

[13] A. K. Schaper, H. Hou, A. Greiner and F. Phillipp, The role of iron carbide in multiwalled carbon nanotube growth. J. Catal. 222, 250-254 (2004).

[14] C. C. Lee, R. G. Sutherland and B. J. Thomson, Hydrogen transfer in the mass spectral fragmentation of cyclohexadienylcyclopentadienyliron. Tetrahedron Lett. 13, 26252626 (1972).

[15] Y. Yin and A. P. Alivisatos, Colloidal nanocrystal synthesis and the organic-inorganic interface. Nature 437, 664-670 (2005).

[16] M. Respaud, J. M. Broto, H. Rakoto, A. R. Fert, L. Thomas, B. Barbara, M. Verelst, E. Snoeck, P. Lecante, A. Mosset, J. Osuna, T. O. Ely, C. Amiens and B. Chaudret, Surface effects on the magnetic properties of ultrafine cobalt particles. Phys. Rev. B 57, 29252935 (1998).

[17] Y. Qiang, J. Anthony, A. Sharma, J. Nutting, D. Sikes and D. Meyer, Iron/iron oxide coreshell nanoclusters for biomedical applications. J. Nanopart. Res. 8, 489-496 (2006).

[18] E. E. Carpenter, S. Calvin, R. M. Stroud and V. G. Harris, Passivated iron as core-shell nanoparticles. Chem. Mater. 15, 3245-3246 (2003).

[19] C. Baker, S. K. Hasanain and S. I. Shah, The magnetic behavior of iron oxide passivated iron nanoparticles. J. Appl. Phys. 96, 6657-6662 (2004).

[20] N. Grobert, W. K. Hsu, Y. Q. Zhu, J. P. Hare, H. W. Kroto, D. R. M. Walton, M. Terrones, H. Terrones, P. Redlich, M. Ruhle, R. Escudero and F. Morales, Enhanced magnetic coercivities in Fe nanowires. Appl. Phys. Lett. 75, 3363-3365 (1999).

[21] V. F. Puntes, D. Zanchet, C. K. Erdonmez and A. P. Alivisatos, Synthesis of hcp-Co nanodisks. J. Am. Chem. Soc. 124, 12874-12880 (2002). 


\section{Chapter 5}

\section{Synthesis and Characterisation of Ruthenium Nanoparticles}

\section{Chapter outline}

\subsection{Introduction}

5.2. Reaction of Bis(2-Methylallyl) 1,5-Cyclooctadiene Ruthenium(II)

5.2.1. Reaction at Room Temperature

5.2.2. Reaction at $40{ }^{\circ} \mathrm{C}$

5.3. Reaction of Ruthenium(III) Acetylacetonate

5.3.1. Effect of Stabilising Agent

5.4. Discussion

5.4.1. Size and Morphological Control

5.4.2. Discussion of Growth Mechanism

5.3. Chapter Summary

5.4. Experimental

5.5. References

\subsection{Introduction}

This chapter describes the synthesis of ruthenium nanoparticles and discusses the effects of different experimental variables leading to size and morphological control of nanoparticles. The main aim of the research was to develop a facile synthesis route for preparing ruthenium nanoparticles using the Fischer-Porter bottle technique. The research also aims to obtain ruthenium nanoparticles of non-spherical, faceted, or branched structures, which could be useful for specific catalytic applications.

Different methods to prepare ruthenium nanoparticles have been reported in the literature. The most commonly adopted solution-phase synthesis is the reduction of ruthenium(III) 
chloride $\left(\mathrm{RuCl}_{3}\right)$ by a reducing agent such as sodium borohydride $\left(\mathrm{NaBH}_{4}\right)$ and propanediol.[1-3] This method has been developed over the years and has been shown to produce uniform ruthenium nanoparticles of 1-7 nm, depending on the reaction conditions. Nevertheless, the purification process is problematic due to either contamination of boron species (from $\mathrm{NaBH}_{4}$ ), or high viscosity of the polyols making it difficult to be separated from the nanoparticles.

Other synthesis methods reported include solvothermal reaction of $\mathrm{RuCl}_{3}$, and decomposition of organometallic compounds of ruthenium. Ruthenium nanoparticles prepared via the former approach are generally unstable and tend to aggregate even during the synthesis. ${ }^{[4]}$ There are more reports of ruthenium nanoparticles prepared from the latter approach, mainly contributed by Chaudret and co-workers.[5-7] Reductive decomposition of $\mathrm{Ru}(\mathrm{COD})(\mathrm{COT})^{*}$ under different conditions had led to ruthenium nanoparticles of spherical, worm-like, and porous structures of various sizes. However, a disadvantage with this method is that preparation of the precursor is laborious, giving low yield and the compound is highly air-sensitive thus requiring very careful handling.

In this study, two commercially available organometallic compounds of ruthenium were investigated: bis(2-methylallyl) 1,5-cyclooctadiene ruthenium(II) complex $\left(\mathrm{Ru}(\mathrm{MA})_{2}(\mathrm{COD})^{\dagger}\right)$, and ruthenium(III) acetylacetonate $\left(\mathrm{Ru}(\mathrm{acac})_{3}^{\ddagger}\right)$.

$\mathrm{Ru}(\mathrm{MA})_{2}(\mathrm{COD})$ was chosen for its relatively low decomposition temperature $\left(\sim 82^{\circ} \mathrm{C}\right)$. It was intended to have a precursor that could react at temperatures below $150{ }^{\circ} \mathrm{C}$, or at room temperature for the synthesis of ruthenium nanoparticles. To the best of our knowledge there is only one account of using this compound as the precursor in preparing ruthenium nanoparticles. Prechtl et al. reported the reaction of this compound in a Fisher-Porter bottle at $50{ }^{\circ} \mathrm{C}$, under hydrogen atmosphere, which produced ruthenium nanoparticles of 2.1-3.5 $\mathrm{nm} .{ }^{[8]}$ The synthesis method is very similar to that adopted in this research project. It was thus of great interest to explore the potential of this compound as a precursor for ruthenium nanoparticle synthesis.

There are many more literature reports of using $\mathrm{Ru}(\mathrm{acac})_{3}$ as the ruthenium source for preparing PtRu alloy nanoparticles, than for ruthenium nanoparticles.[9-11] The only

${ }^{*} \mathrm{COD}=1,5$-cyclooctadiene, $\mathrm{COT}=1,3,5$-cyclooctatriene

$\dagger \mathrm{MA}=2$-methylallyl

$\ddagger$ acac $=$ acetylacetonate 
solution-phase synthesis of ruthenium nanoparticles that used $\mathrm{Ru}(\mathrm{acac})_{3}$ was a solvothermal approach reported by Ghosh et al., in which ruthenium nanoparticles of 2$3 \mathrm{~nm}$ and nanorods of $50 \mathrm{~nm}$ in length were obtained from syntheses conducted at $320^{\circ} \mathrm{C} \cdot[12]$

Despite the lack of literature reporting the use of both compounds in synthesising ruthenium nanoparticles, the results in this study have shown positive outcomes in producing ruthenium nanoparticles of different morphologies and sizes.

\subsection{Reaction of Bis(2-Methylallyl) 1,5-Cyclooctadiene Ruthenium(II)}

The use of $\mathrm{Ru}(\mathrm{MA})_{2}(\mathrm{COD})^{*}$ as a precursor was investigated at room temperature $\left(17-24^{\circ} \mathrm{C}\right)$ and at $40^{\circ} \mathrm{C}$. All reactions were run for 20 hours. For experiments conducted at room temperature, the reaction vessels were placed in a fume cupboard. Different alkylamines and oleic acid were used as the stabilising agent, with toluene as the solvent. The stabiliser to ruthenium ratio was varied between $0.2: 1$ and $10: 1$, while the precursor concentration was varied between 0.01 and $0.1 \mathrm{M}$. Table 5-1 lists the experimental details along with the morphology and size of the nanoparticles obtained.

The effect of temperature can be observed in Table 5-1, with reactions at $40^{\circ} \mathrm{C}$ generally producing nanoparticles of larger size as compared with reactions at room temperature. Figures 5-1 $\mathbf{a}$ and $\mathbf{b}$ show the TEM images of nanoparticles obtained from reactions at room temperature (Expt Ru-01) and at $40{ }^{\circ} \mathrm{C}$ (Expt Ru-08), respectively. Both images were taken at the same magnification and the scale bar in image a applies to both images.

In Figure 5-1c, the SAED pattern acquired from a large ensemble of the nanoparticles of sample Ru-08 was indexed to hcp Ru, which was the only crystalline structure observed. EDS performed on the same area confirmed the sole presence of ruthenium in the sample, as shown in Figure 5-1d. The same electron diffraction pattern was obtained for all the samples characterised. EDS performed on all samples also showed ruthenium being the only element within the samples.

\footnotetext{
${ }^{*} \mathrm{MA}=2$-methylallyl; $\mathrm{COD}=1,5$-cyclooctadiene
} 
Table 5-1. Summary table of experiment with $\operatorname{Ru}(M A)_{2}(C O D)^{*}$ as the precursor.

\begin{tabular}{|c|c|c|c|c|c|c|c|}
\hline Expt & $\begin{array}{c}\text { Conc. } \\
\text { (M) }\end{array}$ & $\begin{array}{l}\text { Stabilising } \\
\text { agent }(\mathbf{S A})^{\dagger}\end{array}$ & SA/Ru & $\begin{array}{l}\text { Temp. } \\
\left({ }^{\circ} \mathrm{C}\right)\end{array}$ & Morphology & $\begin{array}{l}\text { Avg. size } \\
(\mathrm{nm})\end{array}$ & $\begin{array}{l}\text { Nanoparticle } \\
\text { aggregation }\end{array}$ \\
\hline $\mathrm{Ru}-01$ & 0.01 & OLA & 1 & R.T. & Near-spherical & $2.3 \pm 0.5$ & No \\
\hline $\mathrm{Ru}-02$ & 0.1 & OLA & 10 & R.T. & Spherical & $1.9 \pm 0.3$ & Yes \\
\hline $\mathrm{Ru}-03$ & 0.1 & BTA & 10 & R.T. & Spherical & $1.9 \pm 0.3$ & Yes \\
\hline $\mathrm{Ru}-04$ & 0.05 & OLA & 10 & R.T. & Spherical & $1.9 \pm 0.3$ & No \\
\hline $\mathrm{Ru}-05$ & 0.05 & BTA & 10 & R.T. & Spherical & $1.9 \pm 0.3$ & No \\
\hline $\mathrm{Ru}-06$ & 0.05 & OLA & 0.2 & R.T. & Various & $2-10$ & No \\
\hline $\mathrm{Ru}-07$ & 0.05 & BTA & 0.2 & R.T. & Spherical & $2.2 \pm 0.5$ & No \\
\hline $\mathrm{Ru}-08$ & 0.05 & OLA & 1 & 40 & $\begin{array}{l}\text { Spherical \& } \\
\text { triangular plate-like }\end{array}$ & $5.5 \pm 1.5$ & No \\
\hline $\mathrm{Ru}-09$ & 0.05 & DDA & 1 & 40 & $\begin{array}{l}\text { Spherical \& } \\
\text { triangular plate-like }\end{array}$ & $5.5 \pm 1.5$ & No \\
\hline $\mathrm{Ru}-10$ & 0.05 & OLA & 10 & 40 & Near-spherical & $2.5 \pm 0.5$ & No \\
\hline $\mathrm{Ru}-11$ & 0.05 & DDA & 10 & 40 & Near-spherical & $2.5 \pm 0.5$ & No \\
\hline $\mathrm{Ru}-12$ & 0.05 & HDA & 10 & 40 & Near-spherical & $2.5 \pm 0.5$ & No \\
\hline $\mathrm{Ru}-13$ & 0.05 & OLA & 0.2 & 40 & Worm-like & $\begin{array}{l}\text { dia. } 2.5 \pm 0.5 \\
\text { length }>15\end{array}$ & Yes \\
\hline $\mathrm{Ru}-14$ & 0.05 & DDA & 0.2 & 40 & Worm-like & $\begin{array}{l}\text { dia. } 2.5 \pm 0.5 \\
\text { length }>15\end{array}$ & Yes \\
\hline $\mathrm{Ru}-15$ & 0.05 & OLAc & 1 & 40 & No reaction & - & No \\
\hline
\end{tabular}

\# Toluene was used as the solvent for all reactions.

† OLA = oleylamine, BTA = isobutylamine, DDA = dodecylamine, HDA = hexadecylamine, OLAc = oleic acid 

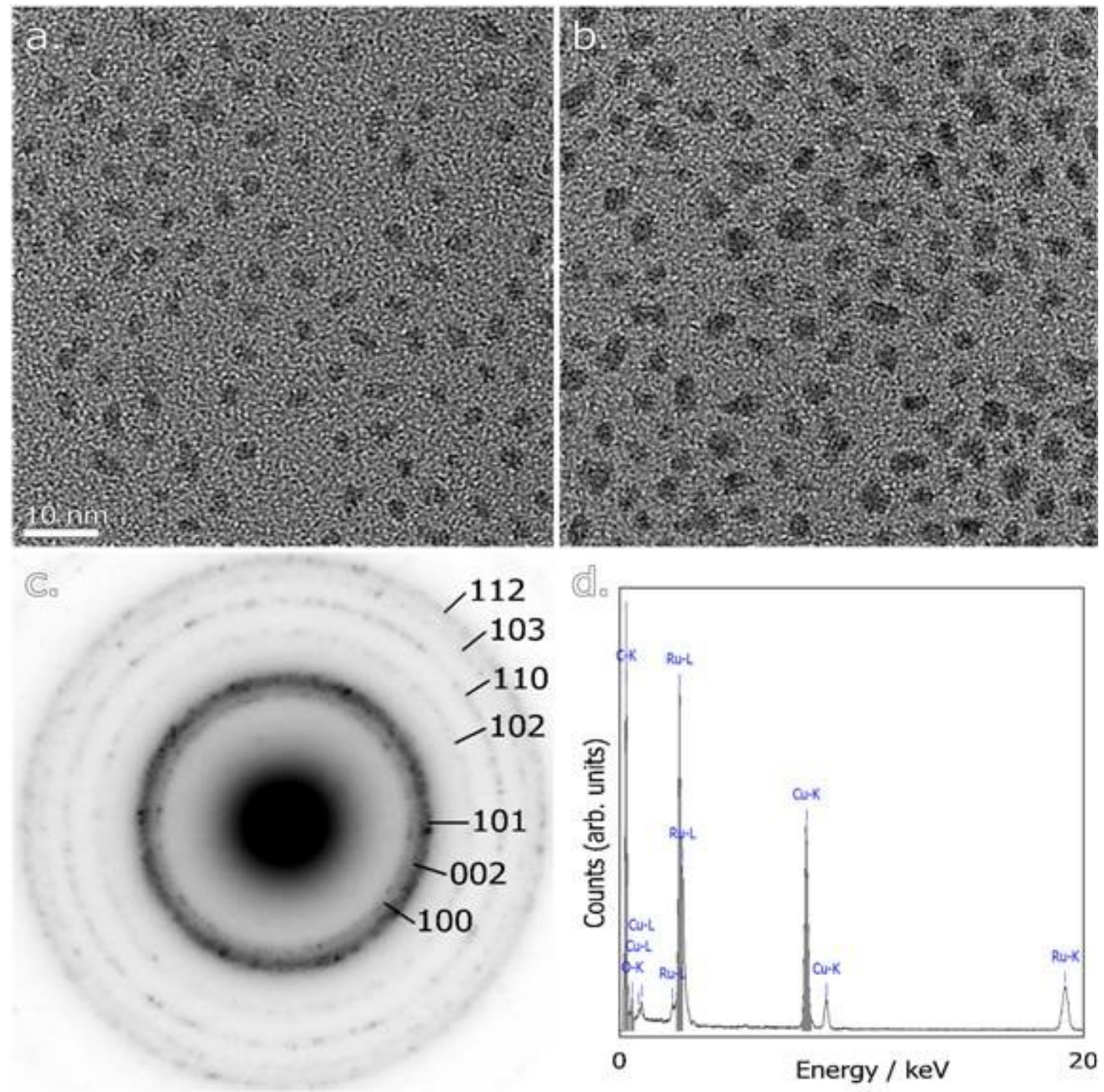

Figure 5-1. Ruthenium nanoparticles formed at different temperatures.

a. TEM image of $2.3 \mathrm{~nm}$ ruthenium nanoparticles obtained at room temperature (Expt Ru-01). b. TEM image of $5 \mathrm{~nm}$ ruthenium nanoparticles obtained at $40{ }^{\circ} \mathrm{C}$ (Expt Ru-08). The scale bar of $10 \mathrm{~nm}$ applies to both images. c. SAED pattern acquired from nanoparticles of sample Ru-08, indexed to hcp Ru. d. EDS showing sole presence of ruthenium in the sample; copper and carbon were from the TEM grid. 


\subsubsection{Reaction at Room Temperature}

At room temperature, reactions were conducted in the fume cupboard. The first reaction produced near-spherical nanoparticles of $2.3 \pm 0.5 \mathrm{~nm}$ with $0.01 \mathrm{M}$ precursor concentration and a 1:1 ratio of oleylamine to ruthenium. A TEM image of the nanoparticles is shown in Figure 5-1a above. When the precursor concentration was increased 10 -fold to $0.1 \mathrm{M}$, and the oleylamine: ruthenium ratio to 10:1 (Expt Ru-02), nanoparticle aggregation was observed, as shown in Figure 5-2a. Close examination of the TEM image indicated that the aggregates comprised individual nanoparticles of $\sim 1.9 \mathrm{~nm}$. Figure $5-2 \mathbf{b}$ shows a TEM image of the nanoparticles obtained from Expt Ru-04, with a precursor concentration of $0.05 \mathrm{M}$ and a 10:1 ratio of oleylamine to ruthenium. The near-monodisperse nanoparticles of $1.9 \pm 0.3 \mathrm{~nm}$ were well stabilised, and were observed to be almost identical to those at the edge of the aggregates in image $\mathbf{a}$. Figures 5-2c and $\mathbf{d}$ show the TEM image of nanoparticles obtained from Expts Ru-06 and Ru-07, respectively. The precursor concentration was $0.05 \mathrm{M}$ for both experiments, and aggregation was not observed. Thus aggregation was shown to be a result of high precursor concentration, as it was not observed for reactions with lower concentrations.

The effect of stabiliser can be observed in Figures 5-2b, c and d. Nanoparticles in image $\mathbf{c}$ (Expt Ru-06) were observed to be bigger than those in image $\mathbf{b}$ (Expt Ru-04), as a result of decrease of oleylamine to ruthenium ratio from $10: 1$ to $0.2: 1$. The nanoparticle size distribution was also larger for the sample with less presence of oleylamine, indicating a less effective nanoparticle stabilisation. The size distribution was improved when the same amount of isobutylamine was used as the stabiliser; spherical nanoparticles of $2.2 \pm 0.5 \mathrm{~nm}$ were obtained, as shown in Figure 5-2d. 

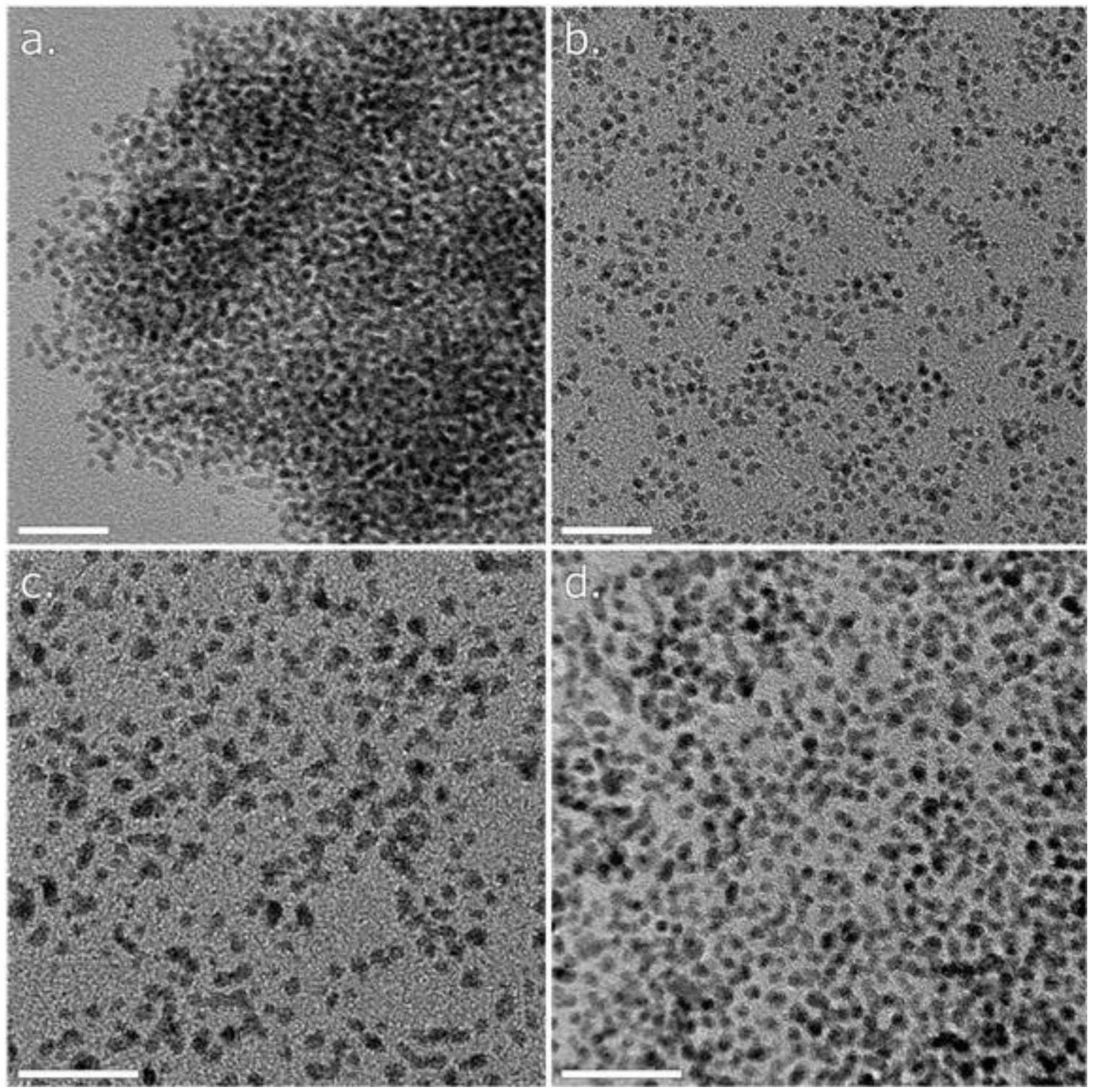

\section{Figure 5-2. TEM images of ruthenium nanoparticles obtained from different reactions} at room temperature.

a. Expt Ru-02, 0.1 M precursor solution, oleylamine to ruthenium ratio $=10: 1$.

b. Expt Ru-04, $0.05 \mathrm{M}$ precursor solution, oleylamine to ruthenium ratio $=10: 1$.

c. Expt Ru-06, $0.05 \mathrm{M}$ precursor solution, oleylamine to ruthenium ratio $=0.2: 1$.

d. Expt Ru-07, $0.05 \mathrm{M}$ precursor solution, isobutylamine to ruthenium ratio = 0.2:1.

All scale bars are $20 \mathrm{~nm}$. 


\subsubsection{Reaction at $40{ }^{\circ} \mathrm{C}$}

The precursor concentration of $0.05 \mathrm{M}$ was used for reactions at $40{ }^{\circ} \mathrm{C}$, as it was found to be ideal for producing monodisperse nanoparticles at room temperature (Section 5.2.1.). In the presence of 10 equivalents oleylamine, near-spherical nanoparticles of $2.5 \pm 0.5 \mathrm{~nm}$ were obtained, as shown in Figure 5-3a. Dodecylamine and hexadecylamine were used in replacement of oleylamine, and the same nanoparticles were observed using TEM.

The effect of the different carbon chain lengths in the alkylamines has not been observed for reactions of different stabiliser to ruthenium ratios of $0.2: 1,1: 1$, and 10:1. Figure 5-3b shows the nanoparticles obtained from Expt Ru-09, with 1:1 ratio of dodecylamine to ruthenium. The nanoparticles were observed to be the same as those formed in the presence of the equivalent amount of oleylamine (Expt Ru-08, as shown in Figure 5-1b). Spherical and triangular plate-like nanoparticles were observed, and were in the size range of $4-7 \mathrm{~nm}$.

When the amount of stabiliser was reduced to 0.2 equivalents, aggregates were observed; nanoparticle aggregation thus indicates the lack of nanoparticle stabilisation. At the edge of the aggregates, nanoparticles of worm-like structures were observed, as shown in Figure 5-3c.

In Expt Ru-15, oleic acid was used as the stabiliser. The reaction solution showed no colour change after 14 hours at $40^{\circ} \mathrm{C}$, indicating that the reaction did not proceed. The reaction was subsequently heated to 60,80 and $96^{\circ} \mathrm{C}$. The colour of the reaction mixture was slightly darker at $80{ }^{\circ} \mathrm{C}$, but a more noticeable change was observed at $96^{\circ} \mathrm{C}$. Dark brown to black precipitate was collected after 5 hours at $96^{\circ} \mathrm{C}$. The nanoparticles were viewed in the TEM and were observed to be polydisperse in both size and shape, as shown in Figure 5-3d. 

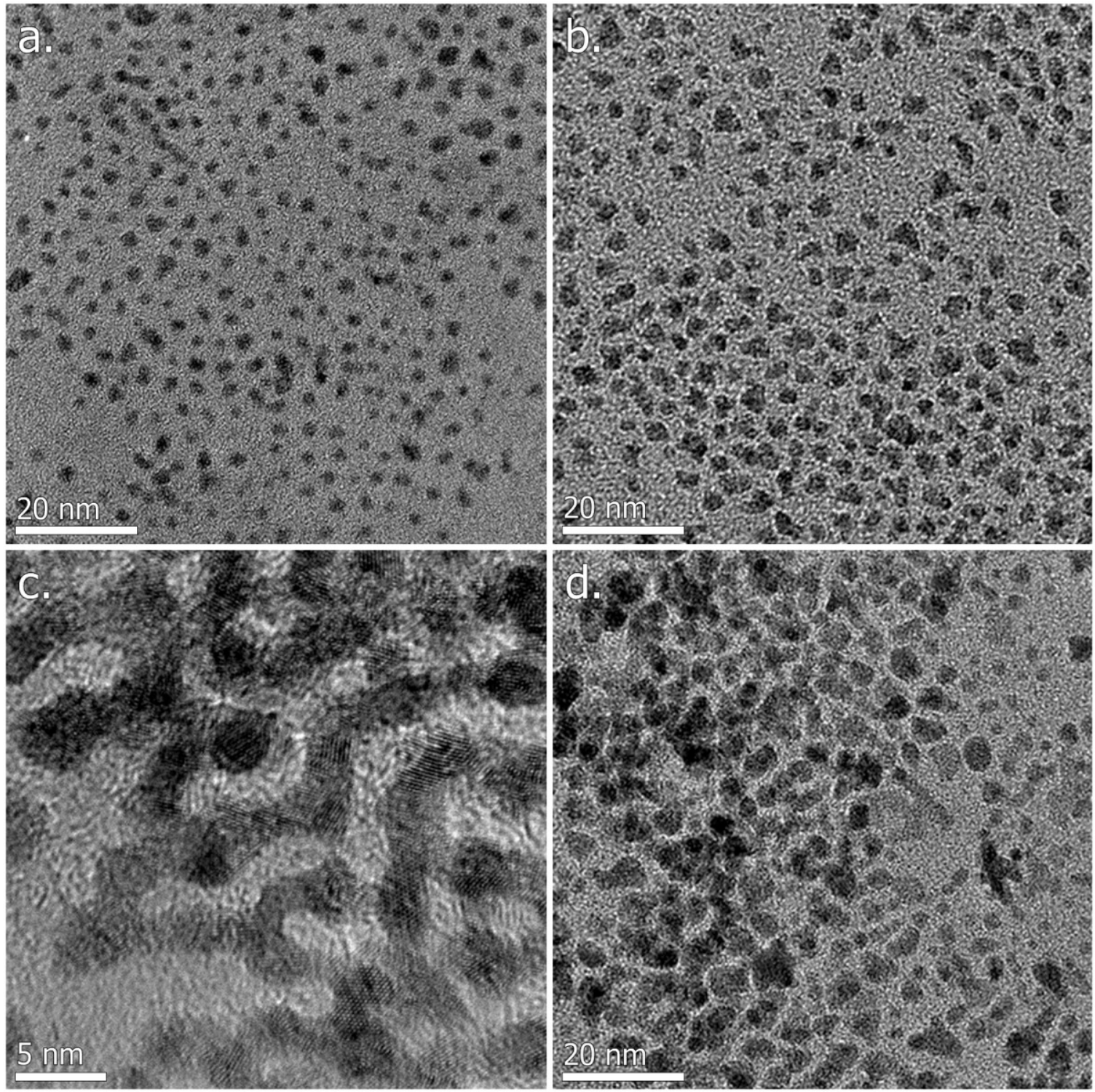

Figure 5-3. TEM images of ruthenium nanoparticles obtained from different reactions at $40{ }^{\circ} \mathrm{C}$.

a. Expt Ru-10, reaction with 10 equivalents oleylamine.

b. Expt Ru-09, reaction with 1 equivalent dodecylamine.

c. Expt Ru-13, reaction with 0.2 equivalent oleylamine.

d. Expt Ru-15, reaction with 1 equivalent oleic acid.

\section{Reaction under Nitrogen Atmosphere}

An experiment was carried out under nitrogen atmosphere to investigate the effect of reaction atmosphere on the decomposition of the precursor. Precursor concentration and amount of oleylamine used were the same as that in Expt Ru-01. The Fischer-Porter bottle was flushed 3 times with nitrogen then filled with atmospheric pressure nitrogen and 
sealed. The Fischer-Porter bottle was first placed in a fume cupboard and then in an oven at different temperatures. Table 5-2 summaries the temperatures employed and the corresponding observations. The pale beige reaction solution remained unchanged between room temperature and $60^{\circ} \mathrm{C}$. Colour change was first observed only once the reaction was heated to $96^{\circ} \mathrm{C}$, indicating decomposition of the precursor. Colour of the reaction solution was light brown after 3 hours at $96^{\circ} \mathrm{C}$, and remained unchanged after 9 hours. The light brown colour observed was much lighter in colour compared to the brown to black colour observed in other experiments, and hence the product was not characterised.

Table 5-2. Reaction conditions and the corresponding observations for an experiment conducted under nitrogen atmosphere.

\begin{tabular}{|c|c|l|}
\hline $\begin{array}{c}\text { Temp. } \\
\left({ }^{\circ} \mathbf{C}\right)\end{array}$ & $\begin{array}{c}\text { Time } \\
(\mathbf{h})\end{array}$ & Observation \\
\hline Room temperature & 20 & No colour change \\
\hline 40 & 11 & No colour change \\
\hline 60 & 6 & No colour change \\
\hline 96 & 9 & Light brown solution \\
\hline
\end{tabular}

\subsection{Reaction of Ruthenium(III) Acetylacetonate}

In this study, an exploratory reaction of ruthenium(III) acetylacetonate was first carried out to determine the lowest temperature required to decompose the precursor in a Fischer-Porter bottle. The precursor solution was prepared with $0.05 \mathrm{M}$ of the precursor in mesitylene, combined with 1.5 equivalents oleylamine as the stabilising agent. Temperatures of $130-140{ }^{\circ} \mathrm{C}$ were found to be suitable for producing highly crystalline ruthenium nanoparticles.

When $\mathrm{Ru}(\mathrm{MA})_{2}(\mathrm{COD}) \S$ was used as the precursor, the most prominent effect observed on the product was observed to be the stabiliser to ruthenium ratio. The approach in this part of the study was thus to examine the effect of stabiliser concentration, by using 0.2 and 10 equivalents of oleylamine. Oleic acid was found to be unsuitable for nanoparticle

$\S \mathrm{MA}=2$-methylallyl; $\mathrm{COD}=1,5$-cyclooctadiene 
synthesis in the reaction of $\mathrm{Ru}(\mathrm{MA})_{2}(\mathrm{COD})$. Nevertheless, when oleic acid was used in replacement of oleylamine in the reactions of ruthenium(III) acetylacetonate, ruthenium nanoparticles of different morphologies were obtained. Table 5-3 lists the experimental details, comparing the different type and concentration of the stabiliser used, along with the morphology and size of the nanoparticles obtained.

Table 5-3. Summary table of experiments with ruthenium(III) acetylacetonate as the precursor.

\begin{tabular}{|c|c|c|c|c|l|l|}
\hline Expt & $\begin{array}{c}\text { Conc. }^{*} \\
\text { (M) }\end{array}$ & $\begin{array}{c}\text { Stabilising } \\
\text { agent (SA) }\end{array}$ & SA/Ru & $\begin{array}{c}\text { Temp. } \\
\left({ }^{\circ} \mathbf{C}\right)\end{array}$ & Morphology & $\begin{array}{l}\text { Avg. size } \\
\text { (nm) }\end{array}$ \\
\hline Ru-16 & 0.05 & OLA & 1 & $70-120$ & Did not characterise & - \\
\hline Ru-17 & 0.05 & OLA & 1.5 & 130 & $\begin{array}{l}\text { Near-spherical } \\
\text { Zigzag, rod-like }\end{array}$ & $\begin{array}{l}5.3 \pm 0.8 \\
\emptyset 3 \pm 0.3, \ell 15 \pm 5\end{array}$ \\
\hline Ru-18 & 0.05 & OLA & 0.2 & 140 & Zigzag, rod-like & $\emptyset 4.5 \pm 1, \ell 40-100$ \\
\hline Ru-19 & 0.05 & OLA & 10 & 140 & Near-spherical & $5.5 \pm 0.5$ \\
\hline Ru-20 & 0.05 & OLAc & 0.2 & 140 & Worm-like & $\emptyset 1.3 \pm 0.2, \ell 9 \pm 5$ \\
\hline Ru-21 & 0.05 & OLAc & 10 & 140 & Spherical & $3.2 \pm 0.2$ \\
& & & & & $\begin{array}{l}\text { Triangular plate-like } \\
\text { Tripod }\end{array}$ & $\begin{array}{l}7.6 \pm 1.5 \\
\emptyset 2.0 \pm 0.5, \ell 7 \pm 2\end{array}$ \\
\hline
\end{tabular}

* Mesitylene was used as the solvent for all reactions.

$¥$ OLA $=$ oleylamine, OLAc $=$ oleic acid

$\dagger \emptyset=$ diameter, $\ell=$ length. For tripod nanoparticles, measurement refers to the size of the arms.

\section{Expt Ru-16. Reaction at temperatures $\leq 120^{\circ} \mathrm{C}$}

In this experiment, the reaction solution was heated in an oven at $70{ }^{\circ} \mathrm{C}$. The reaction solution remained a reddish violet colour after 20 hours, indicating no reaction was taking place. The oven temperature was then increased to $90{ }^{\circ} \mathrm{C}$ and remained for 15 hours, then $100{ }^{\circ} \mathrm{C}$ for 24 hours, and finally at $120^{\circ} \mathrm{C}$ for another 24 hours. At the end of the experiment, the colour of the reaction solution had become slightly darker, which was indicative of decomposition of the precursor and possibly the formation of nanoparticles. However, the slow change of colour suggested a higher temperature was needed for a faster reaction rate and nanoparticle growth. The product was not characterised. 
Table 5-4. Reaction conditions and the corresponding observations for Expt Ru-16 conducted at $\leq 120^{\circ} \mathrm{C}$.

\begin{tabular}{|c|c|l|}
\hline $\begin{array}{c}\text { Temp. } \\
\left({ }^{\circ} \mathbf{C}\right)\end{array}$ & Time & Observation \\
\hline 70 & $20 \mathrm{~h}$ & No colour change \\
\hline 90 & 15 & No colour change \\
\hline 100 & 24 & No colour change \\
\hline 120 & 24 & Dark reddish brown \\
\hline
\end{tabular}

\section{Expt Ru-17. Reaction at $130{ }^{\circ} \mathrm{C}$}

In this experiment, reaction of the precursor was carried out at $130^{\circ} \mathrm{C}$. After 12 hours the reaction solution had turned much darker than that in the previous reaction (Expt Ru-16). The experiment was then carried out for a total reaction time of 3 days. Black precipitate was collected after purification. The supernatant was pale violet in colour, indicating presence of unreacted precursor.

Figure 5-4a shows a TEM image of the nanoparticles obtained. The sample consisted of nanoparticles of near-spherical and rod-like structures. The size of the near-spherical nanoparticles ranged between 3.5 and $10 \mathrm{~nm}$, while the latter had an average diameter of $2.5 \mathrm{~nm}$ and length of $10-20 \mathrm{~nm}$. Both types of nanoparticles are highly crystalline. The SAED pattern acquired from a large ensemble of the nanoparticles was indexed to hcp $\mathrm{Ru}$, as shown in Figure 5-4b. The corresponding EDS in Figure 5-4c shows the sole presence of ruthenium in the sample.

The rod-like nanoparticles were observed to adopt a zigzag pattern, and are hence described as zigzag nanorods. A typical zigzag nanorod is shown in Figure 5-5a. A power spectrum acquired from an area covering the zigzag part of the nanorod (indicated by white square) is shown in Figure 5-5b, which indicates a single crystal structure being projected along a $\langle 100\rangle$ zone axis of hcp Ru (Figure 5-5c). The observation also indicated the nanorod growth was along the $c$-axis of the hexagonal structure. 

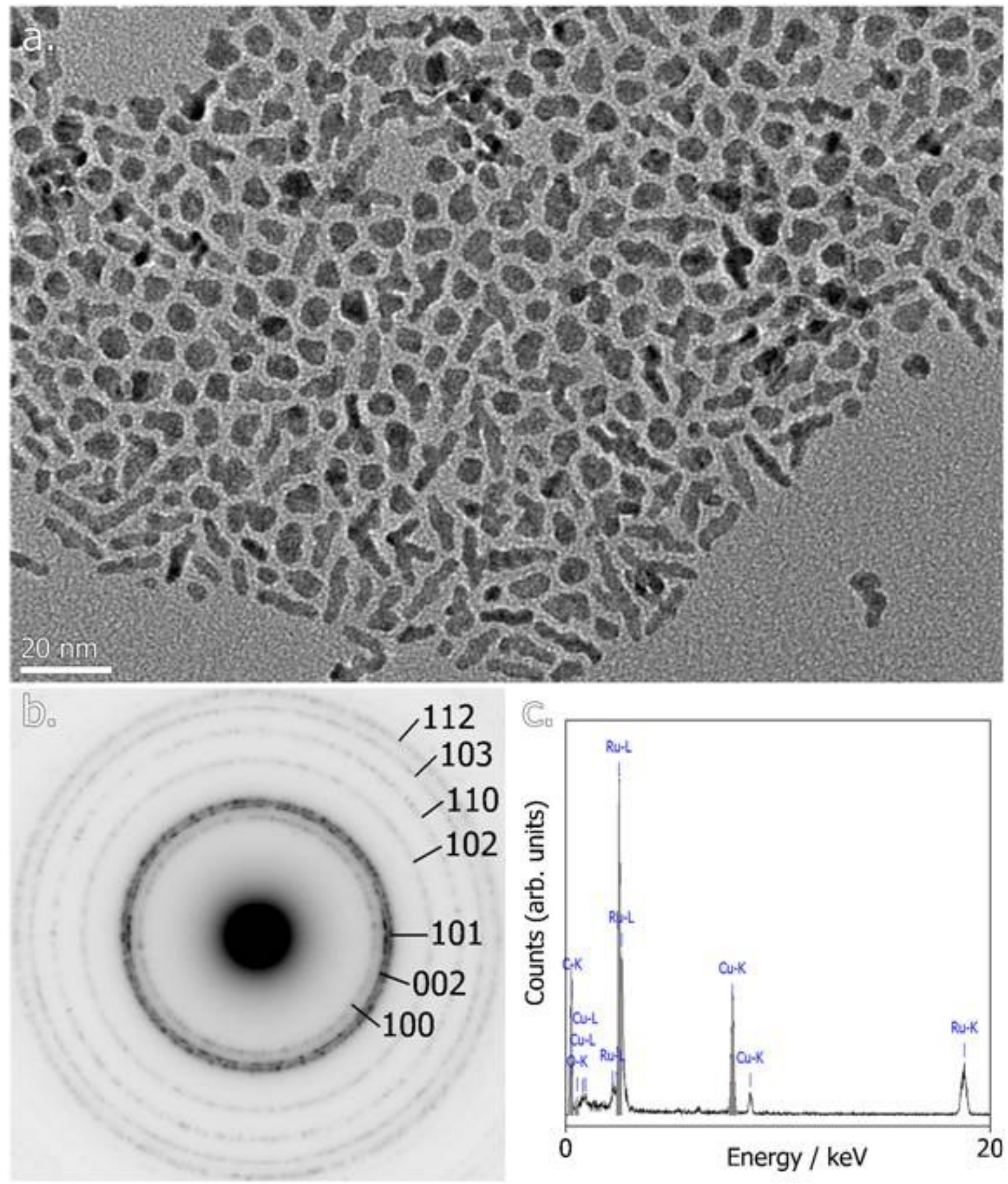

Figure 5-4. Mixture of near-spherical nanoparticles and zigzag nanorods (Ru-17).

a. TEM image of the nanoparticles obtained from Expt Ru-17. b. The corresponding SAED pattern, which can be indexed to hcp Ru. c. EDS showing sole presence of Ru in the sample; $\mathrm{Cu}$ and C peaks were resulted from the TEM grid. 


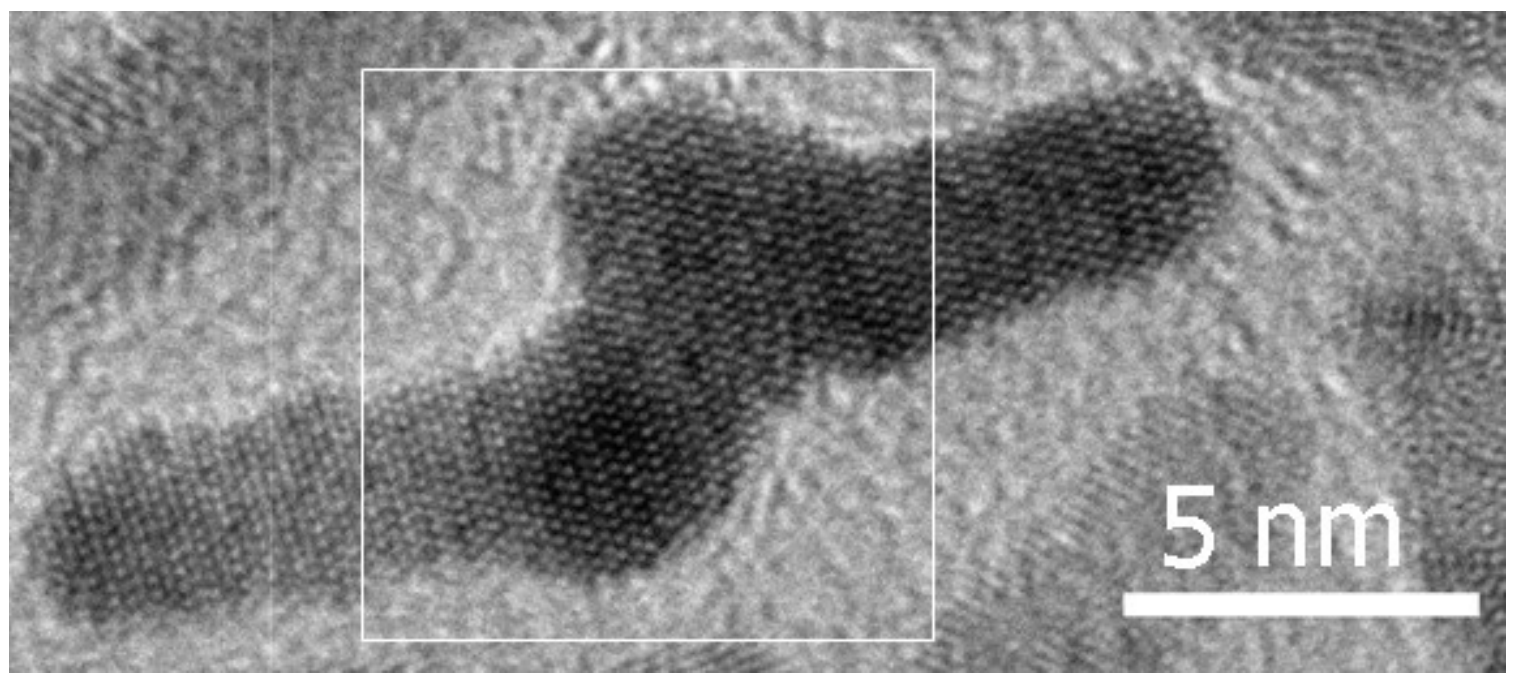

อิ.
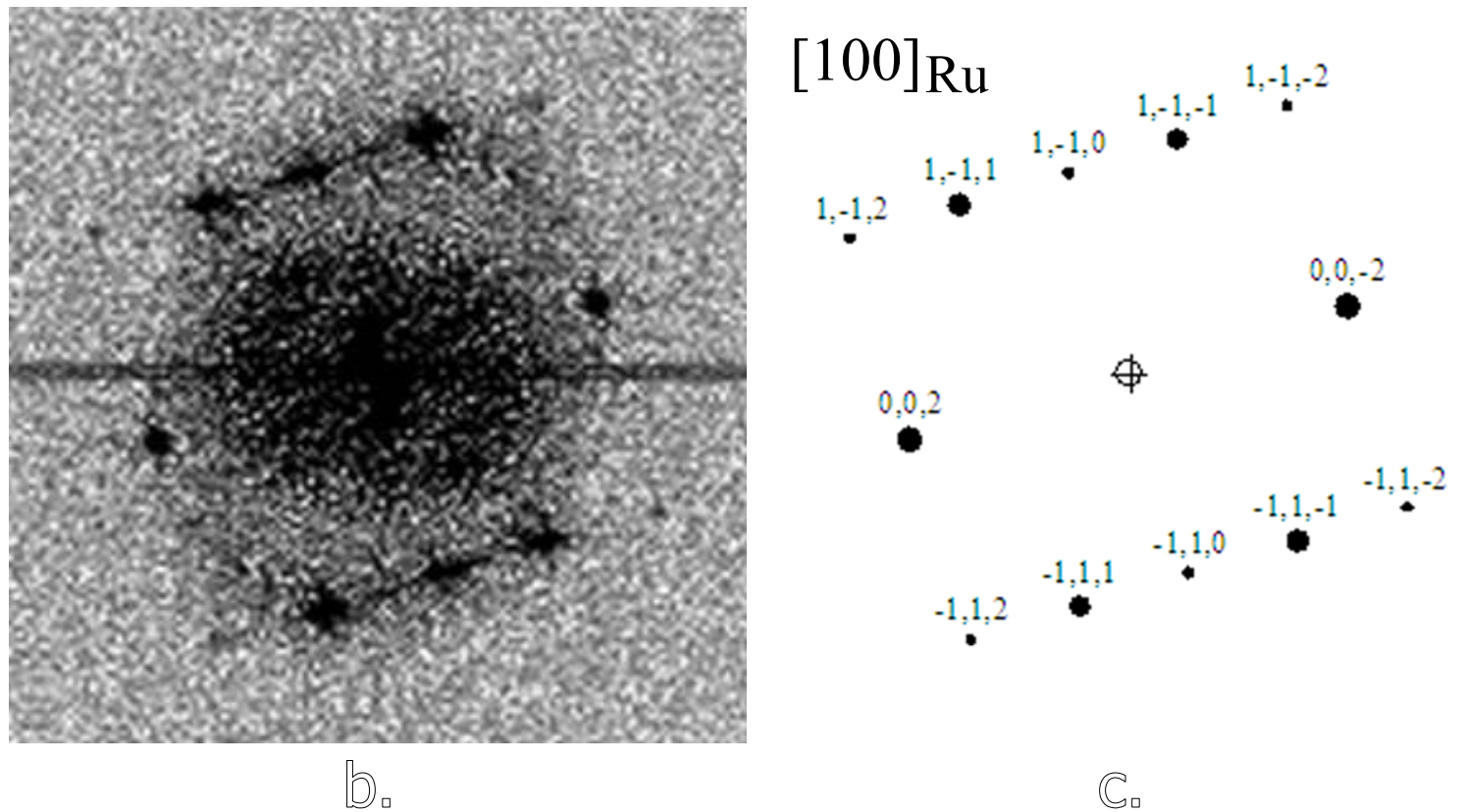

Figure 5-5. HRTEM image of nanoparticles observed in Expt Ru-17.

a. HRTEM image of a typical zigzag nanorod.

b. Power spectrum acquired from an area indicated by the white square in image a, which can be matched to a simulated $\langle 100\rangle$ projection of hcp Ru (c.). 


\section{Reaction under Nitrogen Atmosphere}

An additional experiment was carried out under nitrogen atmosphere to investigate the effect of reaction atmosphere on the decomposition of the precursor. Other reaction conditions were the same as that in Expt Ru-17.

After 3 days, dark brown precipitate was collected after purification. Figure 5-6 shows a TEM image of the nanoparticles obtained. The nanoparticles are of rod-like morphology but are much smaller in size compared to those observed in Expt Ru-17.

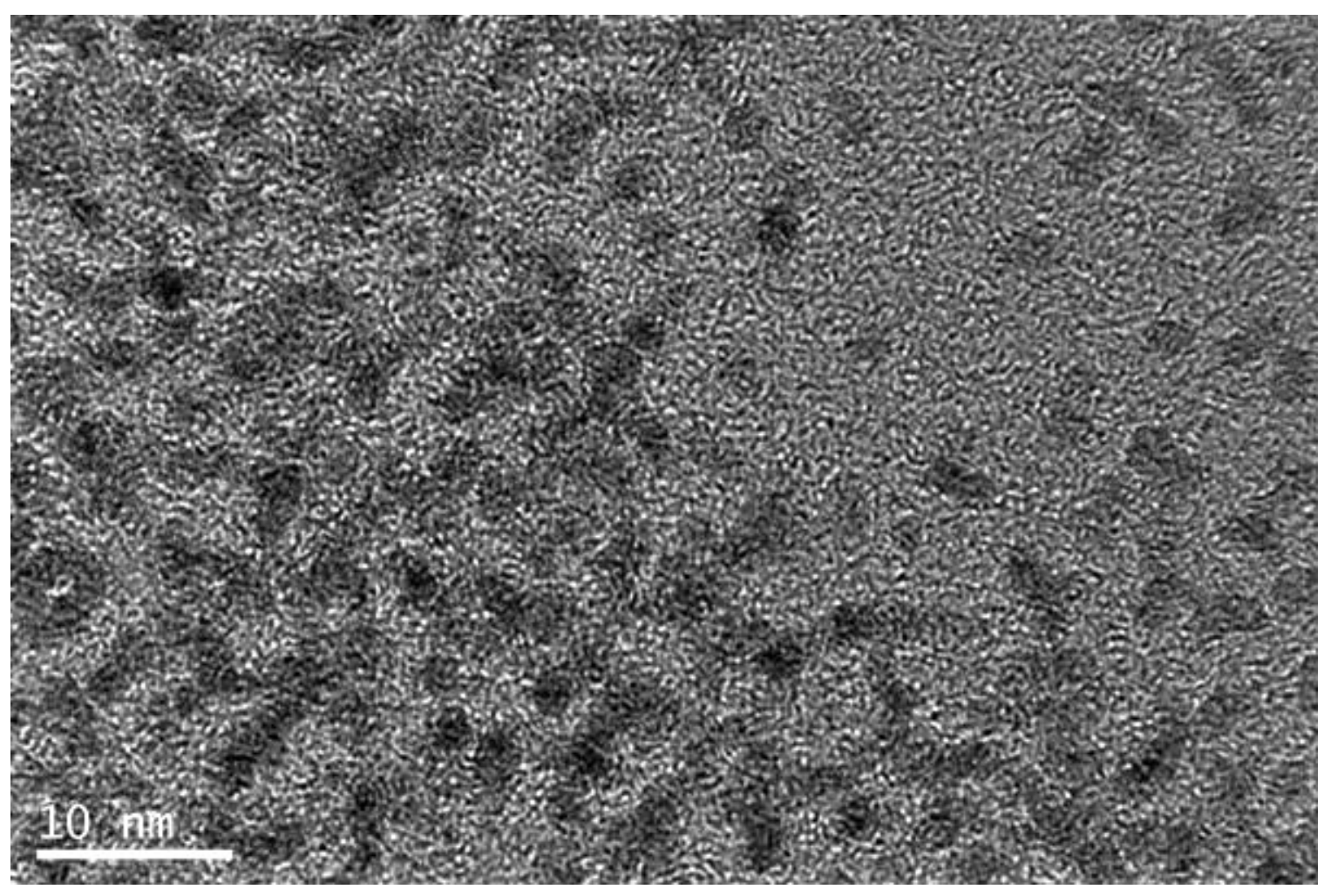

Figure 5-6. TEM image of ruthenium nanoparticles obtained from reaction of ruthenium(III) acetylacetonate under nitrogen.

\subsubsection{Effect of Stabilising Agent}

The effect of the stabilising agent is demonstrated by Expts $\mathrm{Ru}-18, \mathrm{Ru}-20, \mathrm{Ru}-19$, and Ru-21, in which TEM and HRTEM characterisation of the samples are detailed below. As unreacted precursor was indicated in Expt Ru-17, subsequent experiments were carried out at $140{ }^{\circ} \mathrm{C}$ for 60 hours (21/2 days), which yielded brown to black precipitate in colourless supernatant after purification. The colourless supernatant clearly indicated all precursor had reacted. 


\section{Expt Ru-18. Reaction with 0.2 equivalents oleylamine at $140{ }^{\circ} \mathrm{C}$ (low oleylamine concentration)}

The nanoparticles obtained from a reaction with a low oleylamine to ruthenium ratio $(0.2: 1)$ mainly adopted the morphology of the zigzag nanorods, but with a much greater aspect ratio. These nanoparticles have an average diameter of $4 \pm 1 \mathrm{~nm}$, and lengths in the range 40-100 $\mathrm{nm}$. Due to the much higher aspect ratio, these nanoparticles were observed to resemble the structure of a wooden stick, and are thus described as nanosticks. Figure 5-7 shows a TEM image of the nanoparticles obtained. The nanosticks tend to randomly stack onto each other when the nanoparticle suspension dries on a TEM grid. It is possible that some form of aggregation had occurred due to the reduced amount of stabilizing agent present. The isolated nanosticks were observed to be single crystalline structures. TEM image of a typical nanostick is shown in Figure 5-8a. HRTEM image and the corresponding power spectrum showed that the nanostick was a single crystal. A close-up of the nanostick as indicated by the white square is shown in Figure 5-8b, and the power spectrum shown in the inset.

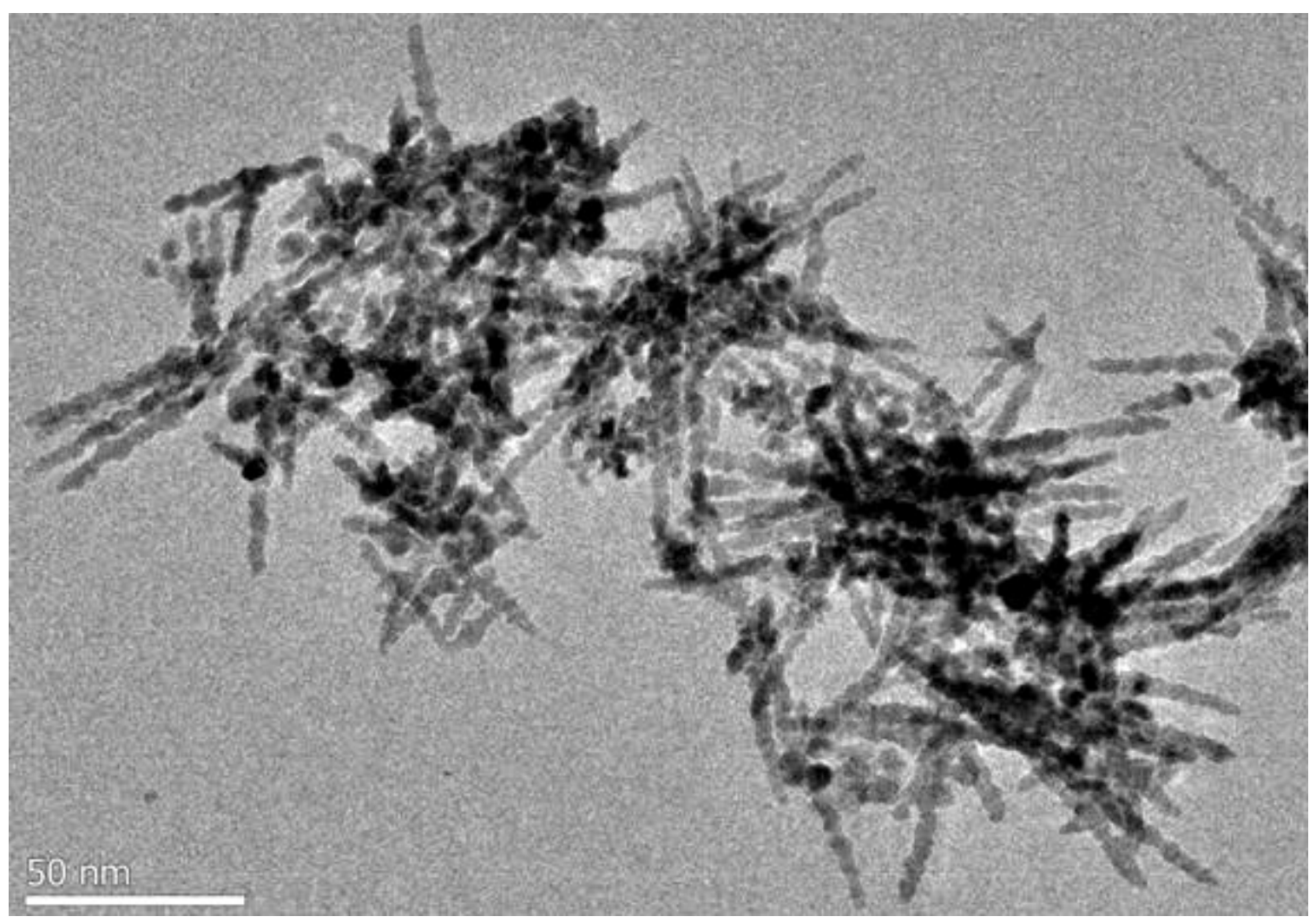

Figure 5-7. TEM image of ruthenium nanoparticles obtained from reaction with low oleylamine concentration. 


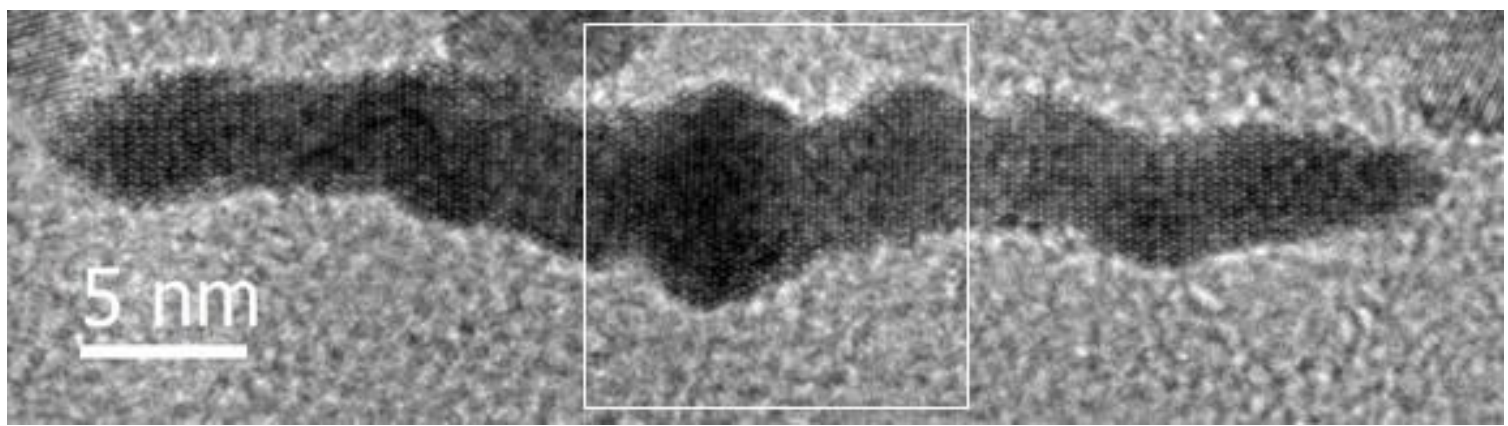

(9).

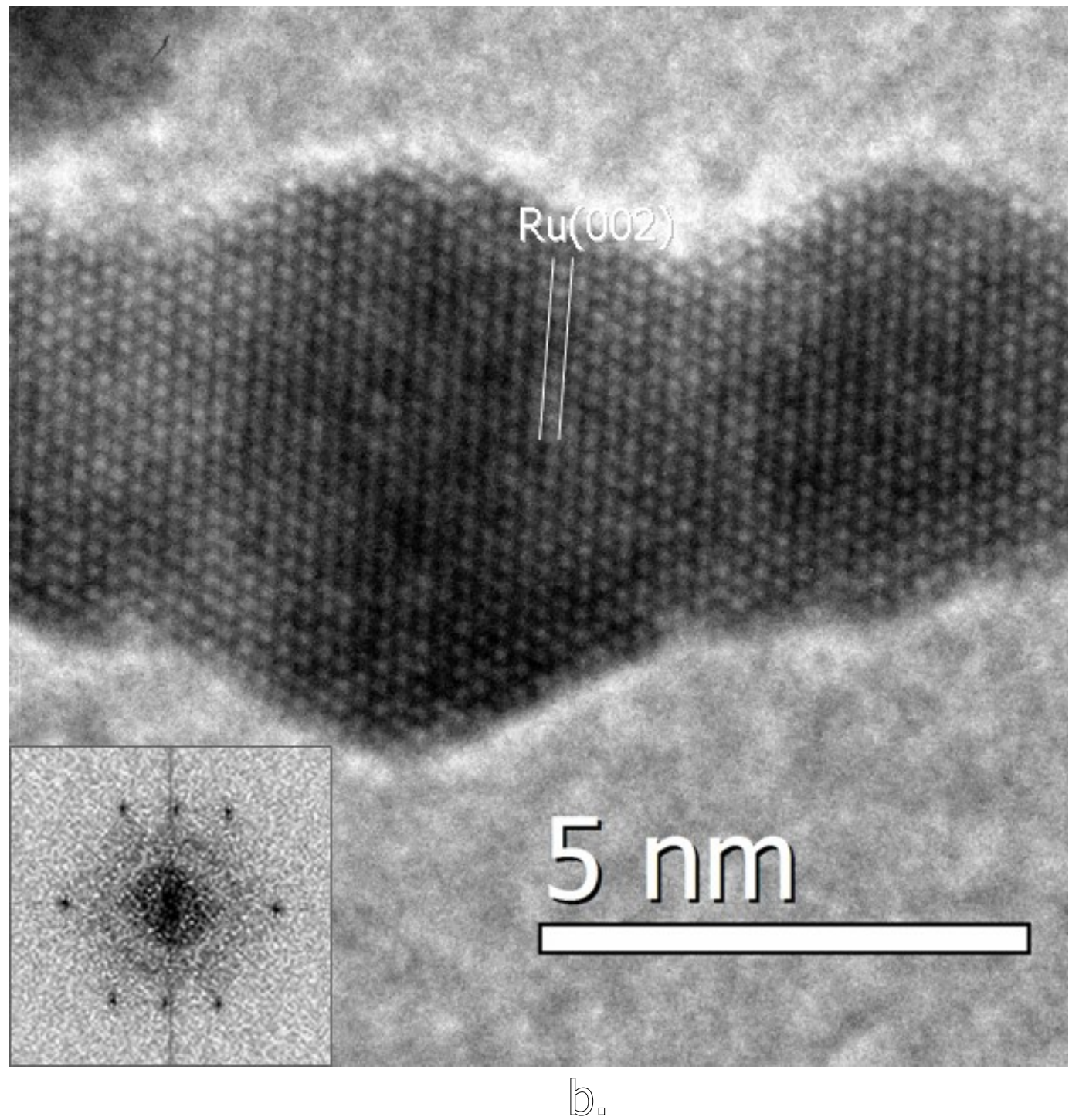

Figure 5-8. HRTEM analysis of a nanostick.

a. TEM image of a typical nanostick. b. HRTEM image of a section of the nanostick (indicated by white square in image a), which shows single crystal stricture. The inset shows the power spectrum of the image, which can be matched to a $\langle 100\rangle$ projection of hcp Ru. The nanostick is thus indicated to be growing along the $\langle 001\rangle$ directions. 


\section{Expt Ru-19. Reaction with 10 equivalents oleylamine (high oleylamine concentration)}
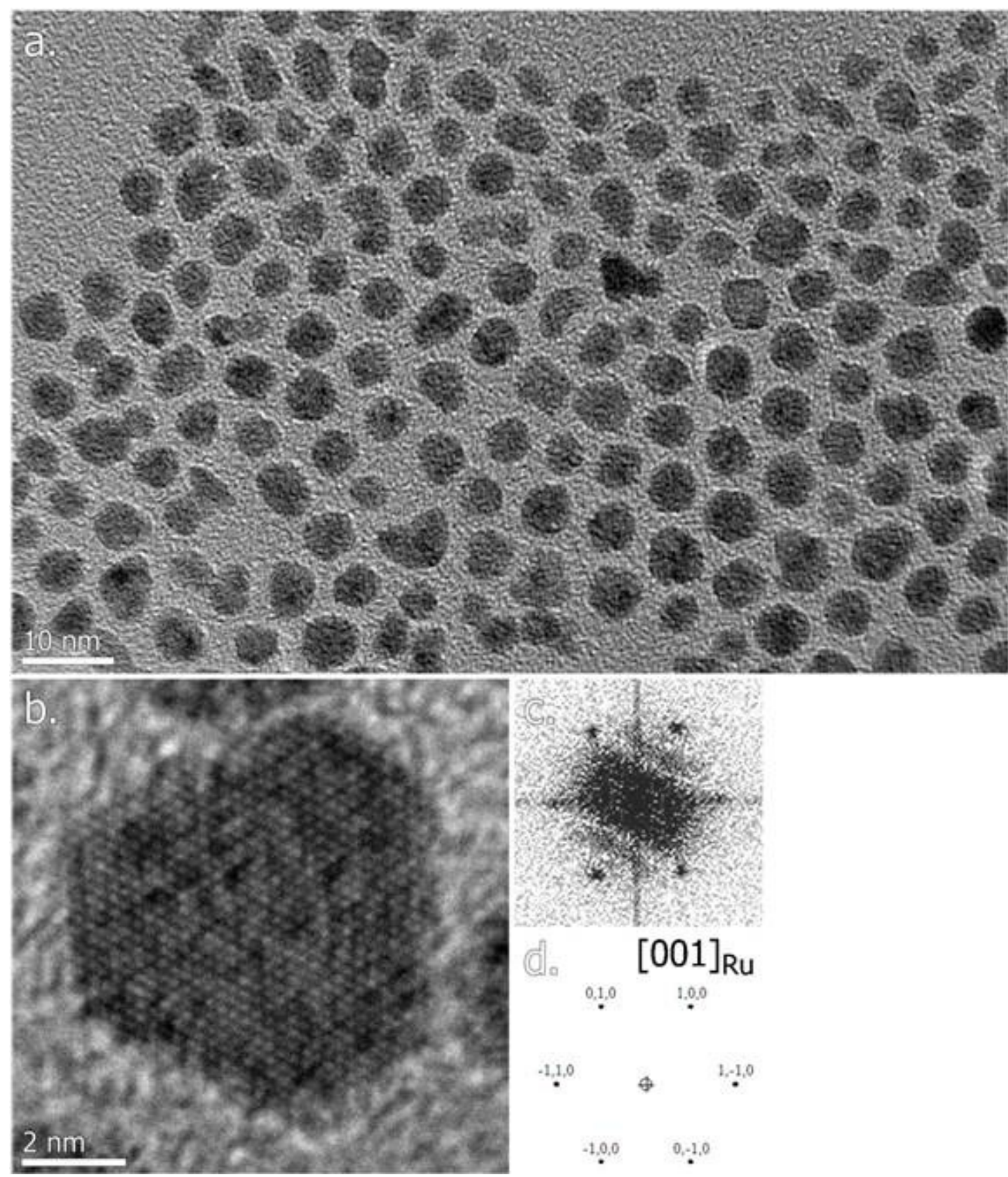

Figure 5-9. Nanoparticles obtained from reaction of high oleylamine to ruthenium ratio.

a. TEM image of the nanoparticles obtained. b. HRTEM image of a faceted, truncated triangular plate-like nanocrystal, with lattice fringes showing a hexagonal symmetry corresponding to the projection along a $\langle 001\rangle_{\mathrm{hcp}}$ direction. c. Power spectrum of image b. d. A simulated [001] projection of hcp Ru. 
At a much higher oleylamine to ruthenium ratio of 10:1, near-spherical ruthenium nanoparticles of average $6 \mathrm{~nm}$ were obtained, as shown in Figure 5-9a.

Close examination of the TEM image revealed that about half of the nanoparticles were spherical and multiply twinned, while the other half were faceted and adopted a hexagonal or a truncated triangular plate-like morphology. Figure 5-9b shows a HRTEM image of a highly faceted nanoparticle, which can be described as a truncated triangular plate-like nanocrystal. The corresponding power spectrum indicates the nanocrystal is being projected along a $\langle 001\rangle$ zone axis of the hcp structure.

As these nanoparticles are near-monodisperse in size, they tend to assemble into a hexagonal array when the nanoparticle suspension dries on the TEM grid. It was then observed in the TEM that these closely assembled nanoparticles sintered under the electron beam. Figure 5-10 shows the TEM images of nanoparticles before (a) and during (b) the sintering was observed, in which groups of 2-3 nanoparticles sinter to form chain-like nanostructures.
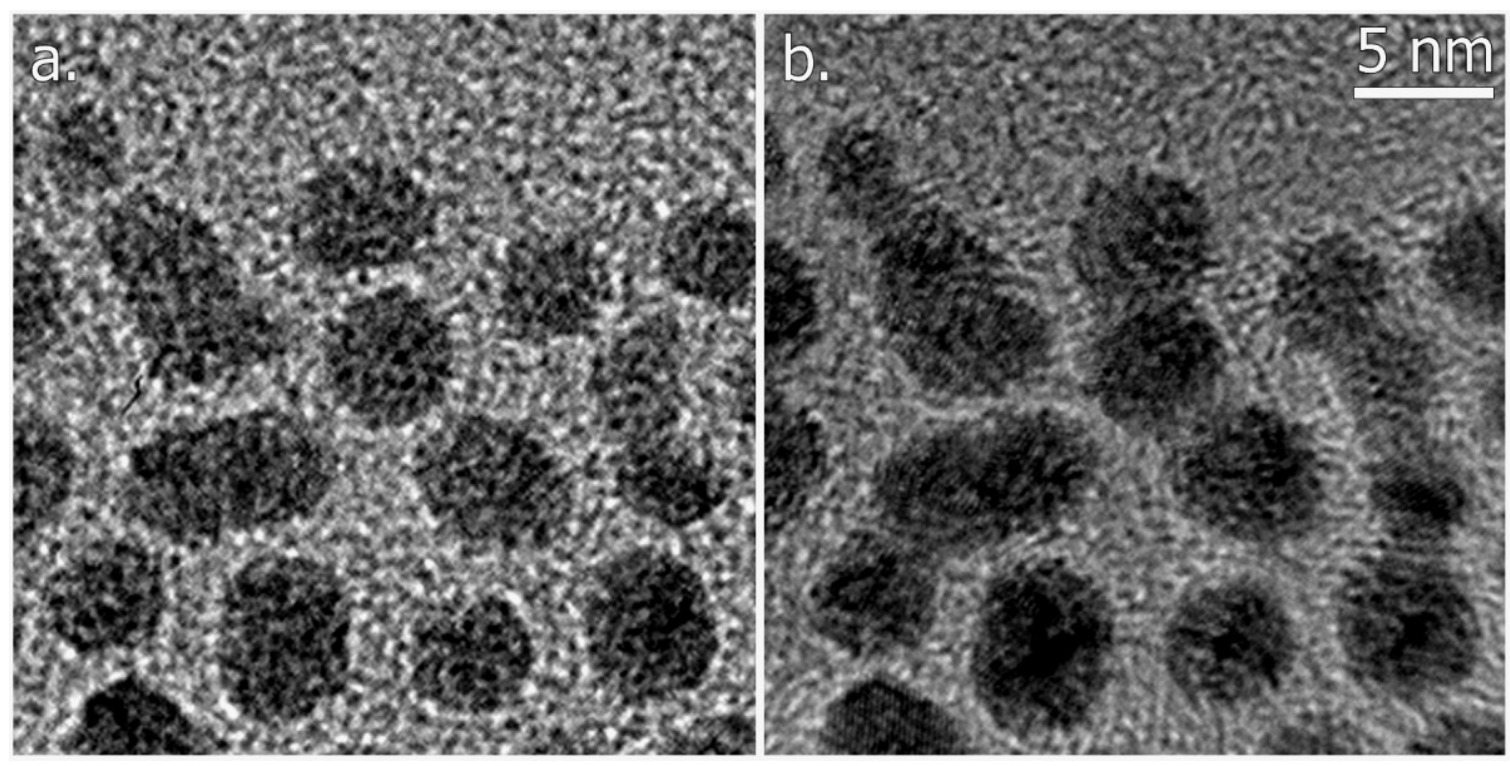

\section{Figure 5-10. Sintering of ruthenium nanoparticles.}

a. TEM image of a group of ruthenium nanoparticles before sintering was observed.

b. TEM image of the same area showing sintering of some nanoparticles. 
Expt Ru-20. Reaction with 0.2 equivalents oleic acid (low oleic acid concentration)
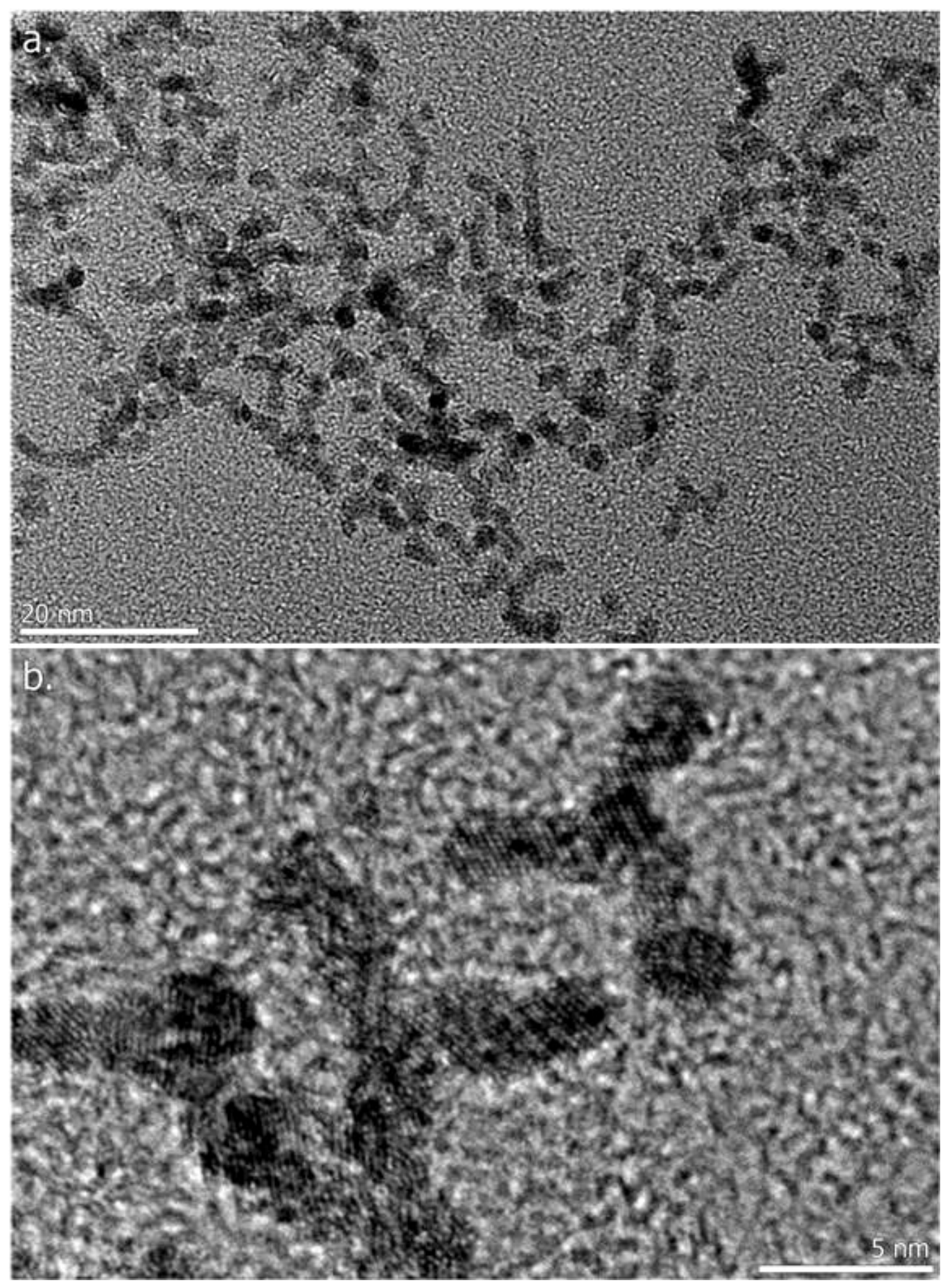

Figure 5-11. Worm-like nanostructures from reaction of low oleic acid:Ru ratio.

a. TEM image of the nanoparticles obtained. b. HRTEM image of worm-like nanoparticles.

A TEM image of the nanoparticles obtained from the reaction with low oleic acid : ruthenium ratio (0.2:1) is shown in Figure 5-11a. The ruthenium nanoparticles 
look similar to a rod-like structure that is curved or curled. These nanoparticles typically have an average diameter of $\sim 2 \mathrm{~nm}$, and lengths varying between 5 and $20 \mathrm{~nm}$. Variations of the worm-like morphology were also observed, including branched and 2-D network of worm-like structures. The nanostructures were observed to be polycrystalline, as can be seen in the HRTEM image in Figure 5-11b.

\section{Expt Ru-21. Reaction with 10 equivalents oleic acid (high oleic acid concentration)}

When the oleic acid amount was increased by 50 -fold, to an oleic acid to ruthenium ratio of 10:1, discrete nanoparticles of well-defined morphology were formed. Figure 5-12 shows a TEM image representative of the sample. Three groups of nanoparticles in terms of morphology are observed: (i) spherical, (ii) truncated triangular plate-like, and (iii) tripod. The ratio of these three groups counted from 200 nanoparticles was i:ii:iii $=2: 1: 1$. The spherical nanoparticles were near-monodisperse in size, averaging at $3.2 \pm 0.2 \mathrm{~nm}$, and tend to assemble into a hexagonal array among themselves when the nanoparticle suspension dries on the TEM grid. Most of these nanoparticles were observed to be polycrystalline.

The truncated triangular plate-like nanoparticles generally have sides 5-10 nm. The tripods typically have three arms of $2 \mathrm{~nm}$ in diameter and lengths of 5-9 $\mathrm{nm}$. Both types of nanoparticles were observed to be single crystals. HRTEM image of a typical truncated triangular plate-like nanoparticle is shown in Figure 5-13a. The corresponding power spectrum in Figure 5-13b indicates a projection along a $\langle 001\rangle$ direction of the hcp structure. The tripod nanoparticles were observed to be single crystalline and had the same $\langle 001\rangle$ orientation as projected by the truncated triangular plate-like nanocrystals. Two different tripod nanocrystals are shown in Figure 5-13d and f., with the power spectra of the images shown in $\mathbf{e}$ and $\mathbf{g}$, respectively. The arms of the tripod nanocrystal in image $\mathbf{d}$ were measured to be $120^{\circ}$ apart, and growth of the $\{100\}$ planes were indicated by the power spectrum. Conversely, the second tripod nanocrystal (image f) was characterised by a 3 -fold symmetry. Two of the arms were observed to be $120^{\circ}$ apart, but the third arm was $30^{\circ}$ closer to one of the other arms. The growth directions of the arms are further discussed in the growth mechanism section (5.4.2.). 


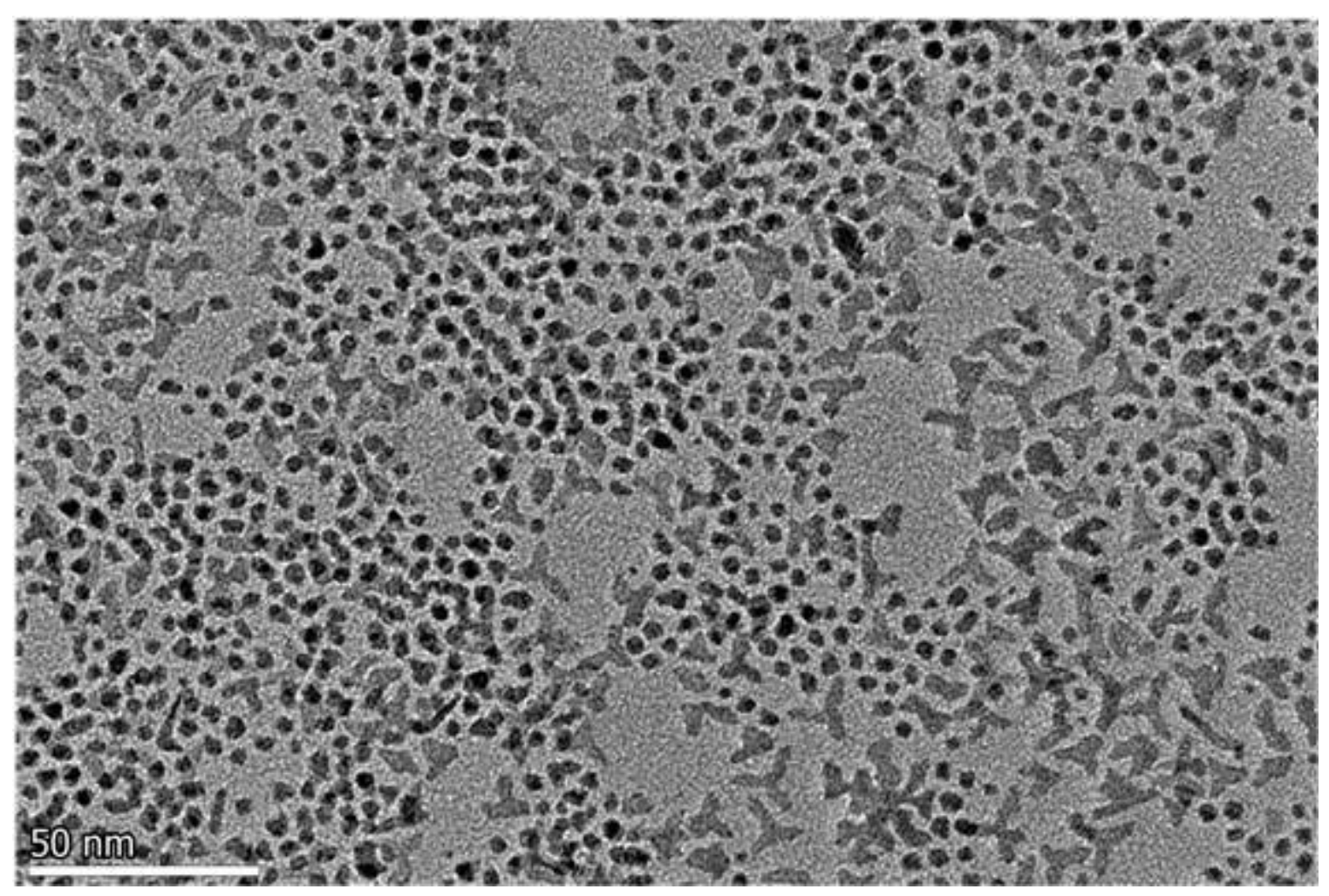

Figure 5-12. TEM image of ruthenium nanoparticles obtained from reaction of high oleic acid concentration. 

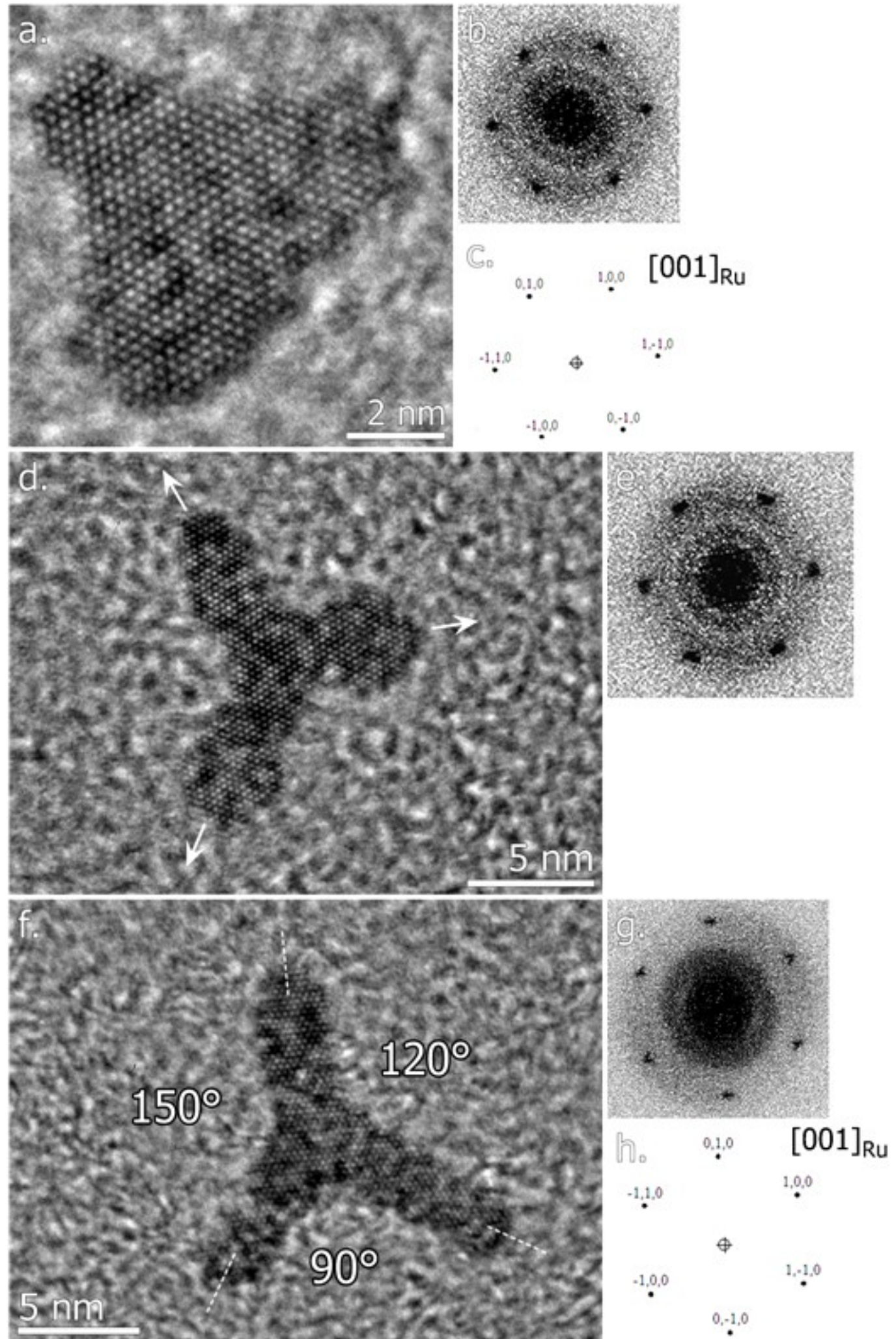

$-1,1$.

$\oplus$

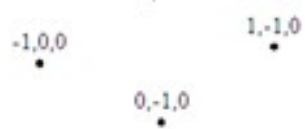

Figure 5-13. HRTEM analysis of triangular plate-like and tripod nanocrystals.

a. HRTEM image of a truncated triangular plate-like nanocrystal. b. Power spectrum of image a. c. Simulated [001] projection of hcp Ru. d. HRTEM image of a tripod nanocrystal. Arrows indicate the growth directions of the arms, which are $120^{\circ}$ apart. e. Power spectrum of image d. f. HRTEM image of a slightly different tripod nanocrystal; not all arms are $120^{\circ}$ apart. g. Power spectrum of image $f$. 


\subsection{Discussion}

In this study, ruthenium nanoparticles of different morphologies were observed. As shown in the results section above, the non-spherical morphologies include 1-dimensional structures such as the zigzag nanorods and worm-like structures, and branched structures such as tripods. While spherical and near-spherical nanoparticles are a result of uniform growth of the respective nuclei, non-spherical nanoparticles are often observed to assume very different growth mechanisms. The conditions leading to the size and morphological control of the nanoparticles are first considered, followed by a discussion on the proposed growth mechanisms of the non-spherical nanoparticles observed.

\subsubsection{Size and Morphological Control}

For spherical and near-spherical morphologies, nanoparticles in the size range of 1.9-5.5 $\mathrm{nm}$ were obtained with small size distribution. Reaction temperature and the amount of stabilising molecules present were observed to be the main factors that impose an obvious effect on the nanoparticle size. These effects are further summarised in Figure 5-14.

As reaction temperature is increased, more energy is supplied to the reaction system. It has been shown that this increases the rates of adsorption and desorption of stabilising molecules on the nanoparticle surface.[13] Since the exchange of the stabiliser on the nanoparticle surface becomes more dynamic, there is a higher chance of free atoms adding onto the nanoparticle surface for growth, leading to larger nanoparticle size.

When the relative stabiliser concentration is increased, there is more stabilising molecules surrounding or around the nanoparticles. If the amount of the stabiliser present is large enough, the surface and surrounding of the nanoparticles could become crowded and addition of free atoms onto nanoparticle surface is restricted. In this situation, free atoms are more likely to come together forming nuclei or clusters followed by minimal growth into nanocrystals of relatively small size. 

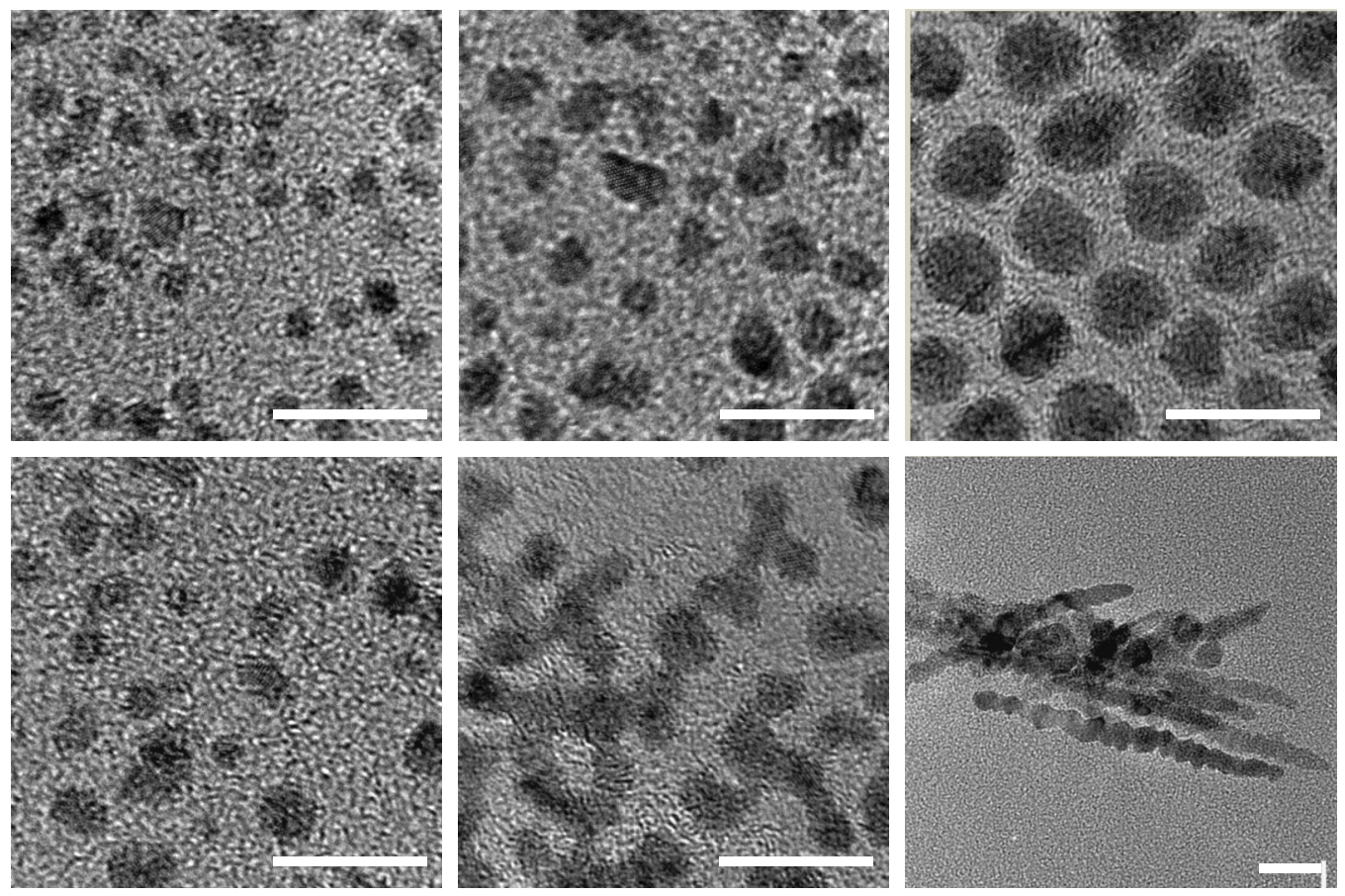

Increase of reaction temperature

\section{Figure 5-14. Effect of temperature and amount of stabiliser on nanoparticle morphology and size.}

Increase of temperature leads to growth of larger nanoparticles; whereas increase of oleylamine (stabiliser) concentration restricts nanoparticle growth results in smaller nanoparticles and of more uniform morphology. All scale bars are $10 \mathrm{~nm}$.

Formation of spherical morphologies was mainly observed for nanoparticles stabilised by alkylamines. This suggests that there is no difference in the adsorption of amine molecules onto the different facets of the nanoparticles, as no effect on the nanoparticle morphology was observed. Although one dimensional structures were observed, the formation occurred in the presence of limited amount of stabiliser, at 0.2 equivalents. It is thus believed that the morphologies were a result of growth along the easy axis (c-axis) of the hcp structure, or aggregation of spherical nanoparticles, instead of growth directed by selective binding of the stabiliser on different facets.

In the presence of oleic acid, ruthenium nanocrystals with tripod structures were observed, compared to near-spherical or plate-like structures observed in the presence of the same amount of oleylamine. This difference indicates the role of oleic acid in the growth of ruthenium nanoparticles. The tripod morphologies are believed to be a result of selective binding. Based on the $\langle 001\rangle$ orientations of the nanoparticles, the carboxylic acid is 
indicated to be binding more strongly onto the $\{001\}$ facets, causing nanoparticle growth to occur in other directions, with branching being one of the options.

\subsubsection{Discussion of Growth Mechanism}

\section{Zigzag nanorods and nanosticks}

Growth along the $c$-axis of hcp ruthenium was observed in both zigzag nanorods and nanosticks. Previous computational and experimental results have shown that morphologies with elongation along the $c$-axis are the most thermodynamically stable shapes for hcp structures.[14]

The formation of similar zigzag structure was first observed in $\mathrm{TiO}_{2}$ nanoparticles by Penn and Banfield.[15] The growth was described to have occurred through the coalescence of faceted nanocrystals in an oriented fashion, such that two high-energy facets are eliminated through each attachment process. The attachment process, often described as oriented attachment, is one of the commonly observed mechanisms in recent literature for the growth of nanorods and nanowires.[16,17] The nanosticks and zigzag nanorods are believed to have formed via the same oriented attachment mechanism. The growth is likely to be promoted by the sequential elimination of the $\{001\}$ facets, which are the higher energy facets. A typical characteristic of the resulting nanoparticles is the presence of defects, which are also observed in the nanosticks.

\section{Worm-like nanoparticles}

Worm-like structures similar to those observed in this study have been previously reported elsewhere. ${ }^{118,19]}$ Although there was no strong evidence from HRTEM imaging to indicate the possible growth mechanisms, the authors proposed the growth as a form of aggregation or random attachment of smaller nanoparticles. Likewise, the observations in this study suggest that the worm-like structures most likely resulted from aggregation of slightly elongated nanoparticles. Among the three types of one-dimensional structures observed in this study, the worm-like nanoparticles are unique in many ways. Unlike the zigzag nanorods and nanosticks, the worm-like nanoparticles are usually not single crystals, and there is no specific direction of growth observed in the elongation of the nanoparticles. From HRTEM imaging, a worm-like nanoparticle typically comprised a few segments with 
each being a single crystal. These segments usually have aspect ratios greater than one, and can be straight or curved. It is believed that these segments were first formed as singlecrystalline nanoparticles that eventually aggregated into worm-like structures.

\section{Tripod and plate-like nanocrystals}

When a hcp crystal structure is projected along the [001] direction, the indexed directions in the lattice are shown in Figure 5-15. From HRTEM imaging, the arms of the singlecrystalline, tripod nanoparticles were indicated to have grown along these specific directions. For a tripod nanocrystal that is characterised by a 3 -fold symmetry, in which the arms are $120^{\circ}$ apart, as shown in Figure 5-13d, these arms are observed to grow along the $[-2-10],[120]$ and $[1-10]$ directions, or the [210], [-1-20] and [-110] directions, respectively. This indicates growth of the $\{10-10\}$ facets. Tripod nanocrystals with arms $90^{\circ}$ and $150^{\circ}$ apart were also observed, as shown in Figure 5-13f. For this type of tripod nanocrystals, growth of the $\{2-1-10\}$ facets is indicated for one of the arms, while the other two arms grow as described above. Hence, growth along one of the [100], [010], or [110] directions is indicated.

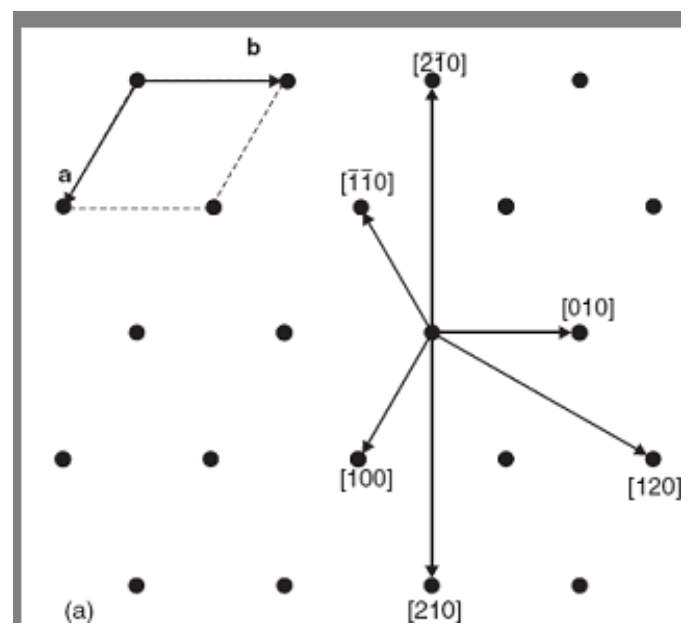

Figure 5-15. Directions in a hexagonal lattice projected along the [001] zone axis.

As discussed earlier, the formation of tripod nanocrystals was under the influence of strong binding of oleic acid onto the $\{0001\}$ planes. The truncated triangular plate-like morphology observed in the same sample is believed to be under similar influence. The nanocrystal in Figure 5-13a is believed to be in the stage of initial branching. The nanocrystal is believed to nucleate into hexagonal or a truncated triangular plate-like 
structure, which is one of the preferred morphologies for hcp crystal structures. This type of morphology is typically bound by $\{0001\},\{10-10\}$, and $\{10-10\}$ planes. Since the growth of $\{0001\}$ is restricted, growth of other planes such as $\{10-10\}$ is thus promoted.

\subsection{Chapter Summary}

Synthesis of ruthenium nanoparticles has been explored and investigated, through a mild and facile solution-phase synthesis method. For all samples characterised, the nanoparticles obtained were shown to be highly crystalline and adopt the hcp structure of bulk ruthenium. A collection of different nanoparticle morphologies and sizes was achieved through the combination of different experimental conditions.

The increase in nanoparticle size was indicated to be a result of increase of reaction temperature and/or decrease of stabiliser to ruthenium ratio. This trend is observed to be independent of the type of surfactant and precursor used.

\subsection{Experimental}

\section{Materials}

All reagents were used as received without further purification. Bis(2-methylallyl) (1,5-cyclooctadiene) ruthenium(II), ruthenium(III) acetylacetonate and oleic acid (reagent grade, $\geq 99 \%$ ) were purchased from Sigma-Aldrich. Oleylamine (technical, $\geq 70 \%$ ) was purchased from Fluka. Toluene, ethanol and methanol were of reagent grade.

\section{Synthesis}

In a typical experiment, $0.1 \mathrm{mmol}$ of a ruthenium precursor was weighed in a vial, then added with toluene as the solvent and oleylamine or oleic acid as the stabilising agent. The vial was placed in a Fischer-Porter bottle, which was then flushed 3 times with hydrogen gas $\left(\mathrm{H}_{2}\right)$ before being filled with $200 \mathrm{kPa} \mathrm{H}_{2}$, and sealed. The bottle was secured in a fume cupboard for room temperature synthesis (Expts Ru-01-Ru-07), or placed into an oven heated at the desired reaction temperature in the range of $40-140{ }^{\circ} \mathrm{C}$. 


\section{Purification}

Reactions carried out at temperatures $40-140{ }^{\circ} \mathrm{C}$ were allowed to cool to room temperature naturally before the Fischer-Porter bottles were opened to air. Equivalent volume of ethanol or methanol was added to the reaction mixture to flocculate and precipitate the nanoparticles. The mixture was centrifuged at $4-10 \times 10^{3} \mathrm{rpm}$ for 5 minutes to isolate a brown to black solid. The solid was re-dispersed in toluene and the precipitation and centrifugation steps repeated. The final product was collected and stored as a powder.

\section{Sample for TEM characterisation}

The samples for TEM studies were prepared by resuspending the precipitate in toluene. One drop of the toluene suspension was put onto a carbon-supported copper grid and allowed to evaporate under ambient conditions.

\subsection{References}

[1] W. Yu, M. Liu, H. Liu, X. Ma and Z. Liu, Preparation, characterization, and catalytic properties of polymer-stabilized ruthenium colloids. J. Colloid Interface Sci. 208, 439444 (1998).

[2] J. Yang, J. Y. Lee, T. C. Deivaraj and H.-P. Too, Preparation and characterization of positively charged ruthenium nanoparticles. J. Colloid Interface Sci. 271, 308-312 (2004).

[3] G. Viau, R. Brayner, L. Poul, N. Chakroune, E. Lacaze, F. Fievet-Vincent and F. Fievet, Ruthenium nanoparticles: size, shape, and self-assemblies. Chem. Mater. 15, 486-494 (2003).

[4] S. Gao, J. Zhang, Y.-F. Zhu and C.-M. Che, A convenient solvothermal route to ruthenium nanoparticles. New J. Chem. 24, 739-740 (2000).

[5] A. Duteil, R. Queau, B. Chaudret, R. Mazel, C. Roucau and J. S. Bradley, Preparation of organic solutions or solid films of small particles of ruthenium, palladium, and platinum from organometallic precursors in the presence of cellulose derivatives. Chem. Mater. 5, 341-347 (1993). 
[6] K. Pelzer, O. Vidoni, K. Philippot, B. Chaudret and V. Collière, Organometallic synthesis of size-controlled polycrystalline ruthenium nanoparticles in the presence of alcohols. Adv. Funct. Mater. 13, 118-126 (2003).

[7] K. Pelzer, B. Laleu, F. Lefebvre, K. Philippot, B. Chaudret, J. P. Candy and J. M. Basset, New Ru nanoparticles stabilized by organosilane fragments. Chem. Mater. 16, 49374941 (2004).

[8] M. H. G. Prechtl, M. Scariot, J. D. Scholten, G. Machado, S. R. Teixeira and J. Dupont, Nanoscale $\mathrm{Ru}(0)$ particles: arene hydrogenation catalysts in imidazolium ionic liquids. Inorg. Chem. 47, 8995-9001 (2008).

[9] P. Sivakumar, R. Ishak and V. Tricoli, Novel Pt-Ru nanoparticles formed by vapour deposition as efficient electrocatalyst for methanol oxidation Part I. Preparation and physical characterization. Electrochim. Acta 50, 3312-3319 (2005).

[10] X. Teng, S. Maksimuk, S. Frommer and H. Yang, Three-dimensional PtRu nanostructures. Chem. Mater. 19, 36-41 (2007).

[11] S. Alayoglu, A. U. Nilekar, M. Mavrikakis and B. Eichhorn, Ru-Pt core-shell nanoparticles for preferential oxidation of carbon monoxide in hydrogen. Nat. Mater. 7, 333-338 (2008).

[12] S. Ghosh, M. Ghosh and C. N. R. Rao, Nanocrystals, nanorods and other nanostructures of nickel, ruthenium, rhodium and iridium prepared by a simple solvothermal procedure. J. Cluster Sci. 18, 97-111 (2007).

[13] S. Sapra, J. Poppe and A. Eychmüller, CdSe nanorod synthesis: a new approach. Small 3, 1886-1888 (2007).

[14] W. A. Miller and G. A. Chadwick, The equilibrium shapes of small liquid droplets in solid-liquid phase mixtures: metallic h.c.p. and metalloid systems. Proc. Roy. Soc. A 312, 257-276 (1969).

[15] R. L. Penn and J. F. Banfield, Morphology development and crystal growth in nanocrystalline aggregates under hydrothermal conditions: Insights from titania. Geochimica et Cosmochimica Acta 63, 1549-1557 (1999).

[16] Y. Yin and A. P. Alivisatos, Colloidal nanocrystal synthesis and the organic-inorganic interface. Nature 437, 664-670 (2005). 
[17] J. H. Yu, J. Joo, H. M. Park, S.-I. Baik, Y. W. Kim, S. C. Kim and T. Hyeon, Synthesis of quantum-sized cubic $\mathrm{ZnS}$ nanorods by the oriented attachment mechanism. J. Am. Chem. Soc. 127, 5662-5670 (2005).

[18] C. Pan, K. Pelzer, K. Philippot, B. Chaudret, F. Dassenoy, P. Lecante and M. J. Casanove, Ligand-stabilized ruthenium nanoparticles: synthesis, organization, and dynamics. J. Am. Chem. Soc. 123, 7584-7593 (2001).

[19] F. Lu, J. Liu and J. Xu, Synthesis of chain-like Ru nanoparticle arrays and its catalytic activity for hydrogenation of phenol in aqueous media. Mater. Chem. Phys. 108, 369374 (2008). 


\section{Chapter 6}

\section{Growth Study of \\ Platinum Nanoparticles in Solution}

\section{Chapter outline}

\subsection{Introduction}

\subsection{Results}

6.2.1. Synchrotron-Based In situ Experiments

6.2.2. Off-Line (Ex situ) Experiments

\subsection{Discussion}

6.3.1. Growth Mechanism: Low-Concentration Reaction

6.3.2. Growth Mechanism: High-Concentration Reaction

6.3.3. The Etching Process in High-Concentration Reaction

6.4. Chapter Summary

6.5 Experimental

6.6 References

\subsection{Introduction}

This chapter presents and discusses the findings obtained from a detailed study of the growth of platinum nanoparticles in solution. The main aim of the research was to investigate the growth of faceted and branched structures of platinum nanoparticles, of which the formation was previously shown to be strongly influenced by precursor concentration. ${ }^{[1,2]}$

In solution-phase synthesis, platinum nanoparticles of spherical or near-spherical structures and polyhedra enclosed by $\{100\}$ and/or $\{111\}$ facets are the most commonly reported.[3-5] Recent studies have also shown the capability to produce unconventional 
structures such as nanoparticles bound by high-index facets, nanowires, multiarmed and porous nanostructures.[2,6,7]

The tendency of platinum nanoparticles to grow into various morphologies has also generated much research interest, especially in the growth of unconventional structures from the highly symmetrical fcc system. Study of the growth mechanism and kinetics governing growth not only contributes to a fundamental understanding of crystal growth at the nanoscale regime, but also provides an essential means to predict the conditions for controlled synthesis needed to tailor the desired properties. Recent reviews in the literature have discussed the profound importance of and challenges in understanding and controlling nanoparticle growth in order to achieve well-defined properties.[8-10] The tested strategies to manipulate growth kinetics, particularly those of metal nanoparticles, include selective capping by suitable stabilising agents, controlling the rate of decomposition or reduction of metal precursors, and deliberate introduction of an etching mechanism.[11-13] The understanding of these concepts has mainly been developed through ex situ studies by quenching the growth process at different reaction times and examining the intermediate products using XRD, TEM, and spectroscopy techniques. Kinetic studies based on an in situ, or real time, approach are relatively recent and have mainly concentrated on nanocrystal growth in terms of crystallite size changes but not considered the shape evolution during the growth process.[14-16]

In this study, synchrotron-based XRD was employed to study the growth of platinum nanoparticles in real time and to provide a direct assessment of the crystallinity, nanocrystal concentration, and crystallite size of the product. TEM was then used to investigate the nanoparticle morphology at different growth stages. By combining the results and observations from both in situ and ex situ studies, the different growth mechanisms associated with thermodynamic and kinetic growth regimes are elucidated and discussed. 


\subsection{Results}

\subsubsection{Synchrotron-Based In situ Experiments}

The experiment involved thermal decomposition of a platinum precursor in a Fischer-Porter bottle in the presence of oleylamine as the stabilising agent, under a hydrogen atmosphere. Two precursor concentrations were employed in this study, $0.005 \mathrm{M}$ and $0.05 \mathrm{M}$ platinum(II) acetylacetonate $\left(\mathrm{Pt}(\mathrm{acac})_{2}\right)$ in toluene, and are labelled as low and high concentration, respectively.

The in situ experiments were conducted in a custom-made XRD cell. Diffractograms were collected with $2 \theta$ values coinciding with the $\operatorname{Pt}(111)$ and $\operatorname{Pt}(200)$ peaks. Growth of the diffracted Pt(111) and Pt(200) peaks was observed and monitored every 5-10 minutes, for up to 1080 minutes (18 hours).

\section{Expt Pt-1. Low-concentration reaction $\left(0.005 \mathrm{M} \mathrm{Pt}(\mathrm{acac})_{2}\right)$}

For the low-concentration reaction, the experiment was terminated after 1080 minutes (18 hours). As shown by the in situ XRD plots in Figure 6-1, no peaks were initially observed, most likely due to the initial crystallites being too small to be observable and/or there being a short induction time before nanoparticle nucleation. As the reaction proceeded, small peaks corresponding to $\operatorname{Pt}(111)$ and $\operatorname{Pt}(200)$ began to emerge and progressively grow over time. The initial peaks were generally broad, especially when they first appeared, indicating the formation of small crystallites. As the reaction continued, the peak area increased indicating an increase in the concentration of nanocrystalline platinum ([ $\left.\left.\mathrm{Pt}_{\mathrm{NC}}\right]\right)$ within the (constant) diffracting volume, and the peak became narrower indicating an increase in the average crystallite size.

From the peak positions, the lattice parameters were calculated. The difference between the calculated and the bulk lattice values, $\Delta d$, was plotted against time, as shown in Figure 6-2. The $\Delta d$ values were observed to vary between -0.01 and $0.01 \AA$ for peaks scanned during the first 120 minutes, after which $\Delta d$ remained at $-0.002 \pm 0.001 \AA$. The calculated lattice parameters were thus close to the bulk showing little or no strain within the nanocrystal domains. 
The area integrated under the $\operatorname{Pt}(200)$ peak had the same profile as that obtained from the $\operatorname{Pt}(111)$ peak, and both profiles were identical after being normalized to the final maximum value, as shown in Figure 6-3. The peak area increased approximately linearly during the first 300 minutes of the experiment, and then increased in a more gradual, logarithmic, fashion through the end of the experiment. The same figure also shows a time-dependent plot of the X-ray correlation length $(L)$ calculated from the fwhm of each peak, which provides an approximation of the crystallite size over time. The $L$ value increased rather rapidly from $\sim 3$ to $8 \mathrm{~nm}$ during the first 250 minutes and remained almost constant throughout. As a result, the growth in low concentration reaction is divided into two stages: I and II, with Stage II begins at 250 minutes, from which $L$ was observed to remain almost constant at $8.4 \pm 0.3 \mathrm{~nm}$.

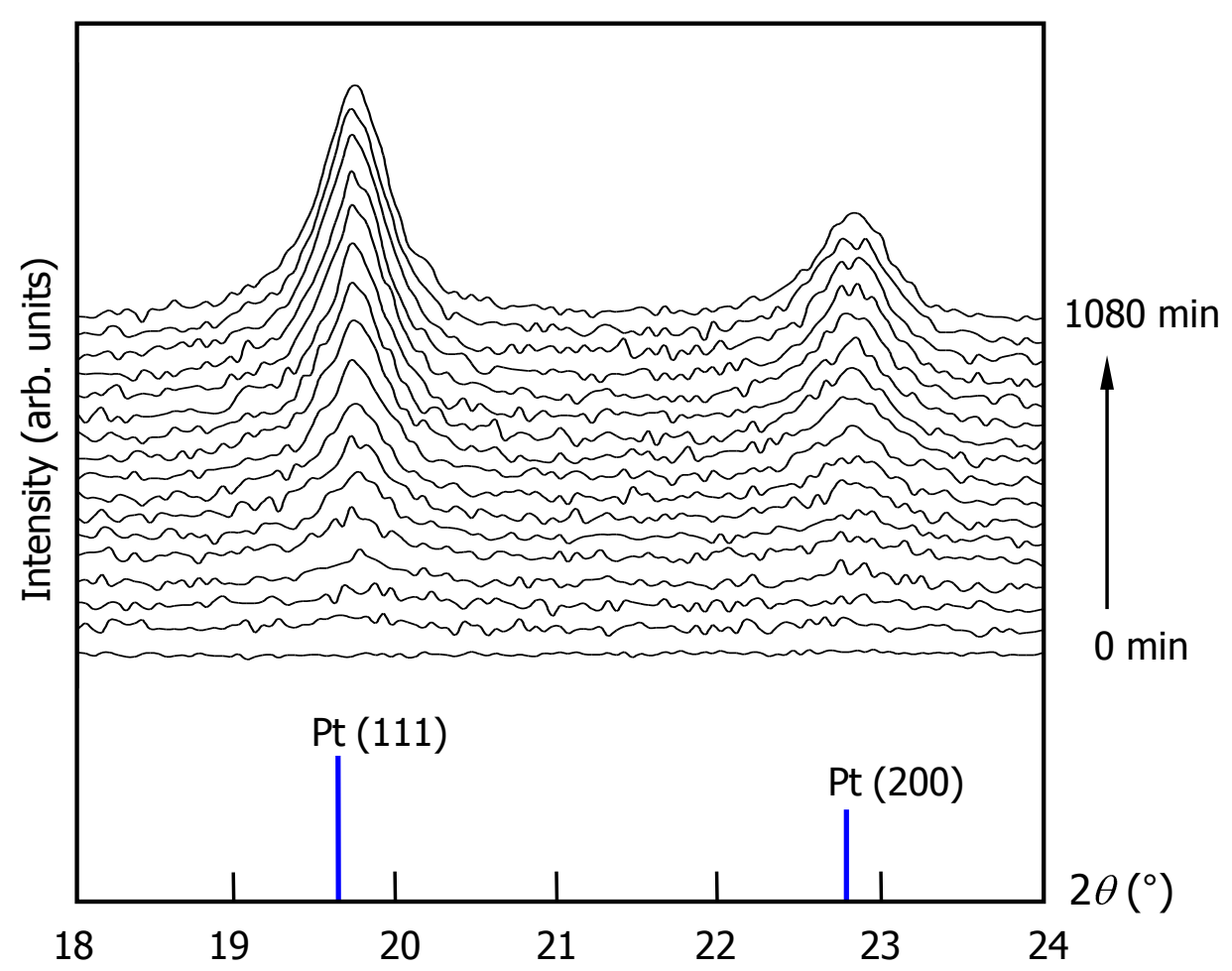

Figure 6-1. In situ XRD plot for low-concentration reaction.

Growth of $\mathrm{Pt}(111)$ and $\mathrm{Pt}(200)$ peaks over 1080 minutes; the respective positions of the (111) and (200) reflections for bulk fcc platinum are shown by blue lines at the bottom of plot. 


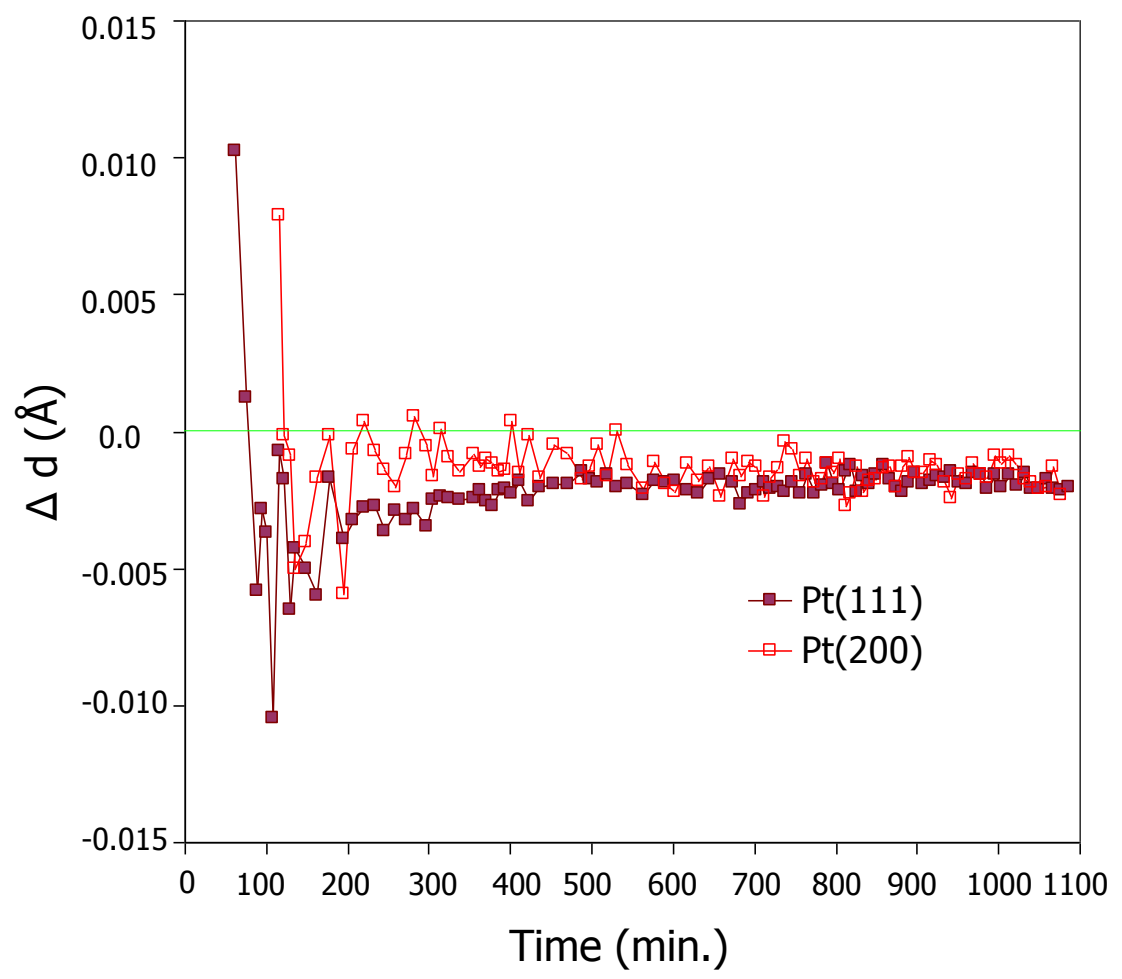

Figure 6-2. Shift in the calculated $d$-spacing from the bulk value for the low-concentration reaction.

$\Delta d=d_{\text {calculated }}-d_{\text {bulki }}$ the green line across at $\Delta d=0$ indicates $d=2.26$ and $1.96 \AA$, corresponding to bulk Pt(111) and Pt(200), respectively.

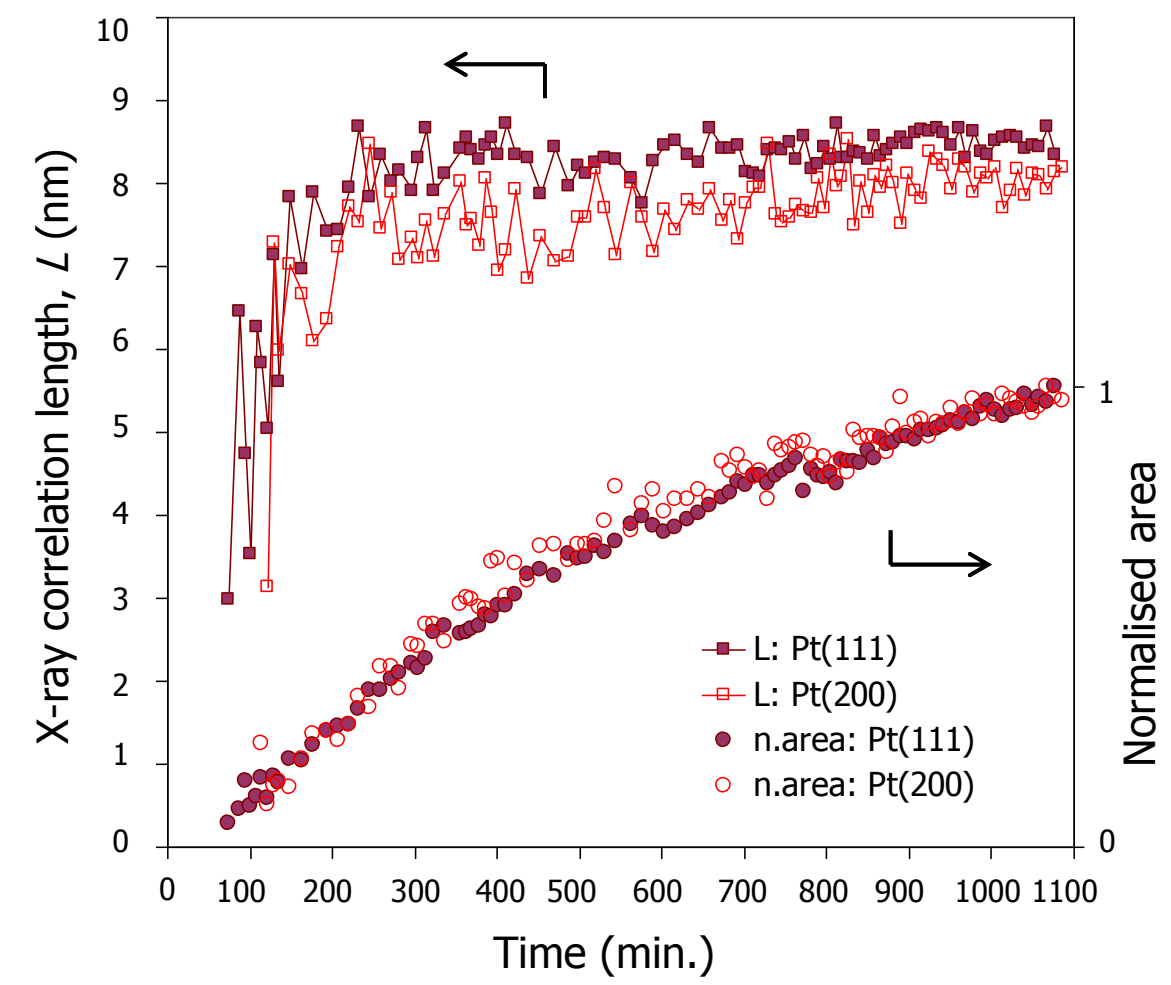

Figure 6-3. Normalised area and X-ray correlation length against time for the low-concentration reaction. 
The product obtained at the end of the in situ experiment was characterised by TEM. As shown in Figure 6-4, the product collected comprised faceted nanoparticles of irregular shapes. These nanoparticles were single-crystalline, with an average size of $11 \pm 3 \mathrm{~nm}$, consistent with the X-ray correlation length shown in Figure 6-3.

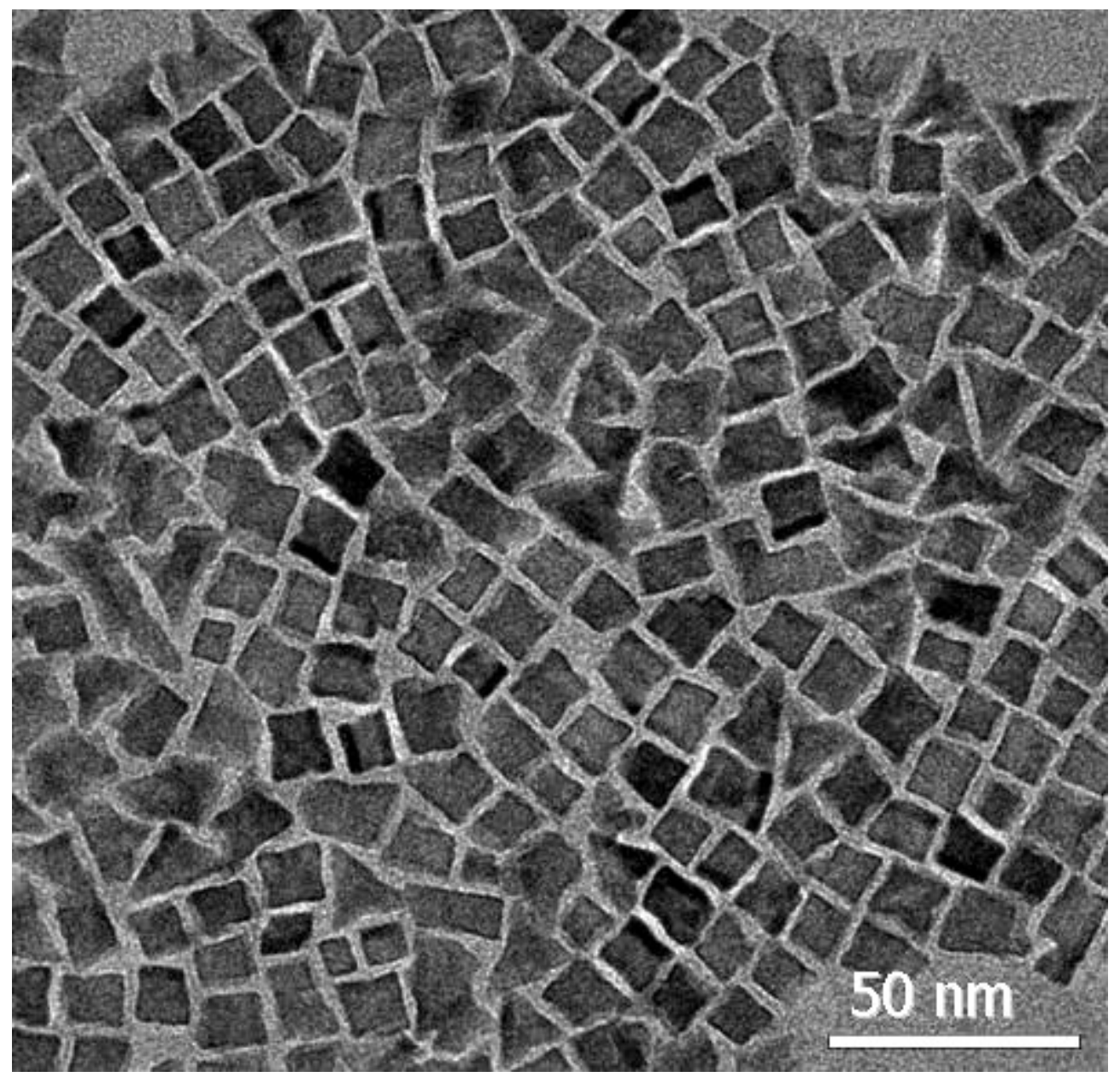

Figure 6-4. TEM result of the in situ experiment of low precursor concentration (Pt-1).

\section{Expt Pt-2. High-concentration reaction $\left(0.05 \mathrm{M} \mathrm{Pt}(\mathrm{acac})_{2}\right)$}

The high-concentration experiment was terminated after 900 minutes (15 hours), and the in situ XRD plots are shown in Figure 6-5. No peaks were initially observed, and small peaks corresponding to $\operatorname{Pt}(111)$ and $\operatorname{Pt}(200)$ began to emerge and progressively grew as the reaction proceeded, which was similar to that observed for the low concentration reaction. As shown in Figure 6-6, the lattice parameters calculated from the peak positions were close to the bulk, again showing little or no strain within the nanocrystal domains. The 
difference between the calculated and the bulk lattice values was determined to be $-0.004 \pm 0.001 \AA$ in average.

Figure 6-7 shows that both the normalised peak area and X-ray correlation length $(L)$ derived from the $\operatorname{Pt}(200)$ peak were almost identical to that from the $\operatorname{Pt}(111)$ peak. As compared with that observed for the low-concentration reaction, the peak area plot for the high-concentration reaction was more complex and could be divided into four stages based on the slope of the plot. For better comparison between the two reactions, the peak area under Pt(111) (not normalised) of both reactions were plotted against time, as shown in Figure 6-8.

For the first $\sim 60-100$ minutes of growth, denoted as Stage I, the peak area for the high-concentration reaction increased rapidly and linearly, with a slope that is ten times greater than that of the low-concentration plot. This indicated a 10 -fold increase in the growth rate of $\left[\mathrm{Pt}_{\mathrm{NC}}\right]$ in the high-concentration reaction compared to that in the low-concentration reaction. Remarkably, between $\sim 100$ and 180 minutes, denoted as Stage II, the peak area remained almost constant, which showed almost no growth in [ $\left.\mathrm{Pt}_{\mathrm{NC}}\right]$. From 180-300 minutes, denoted as Stage III, the peak area resumed with a more rapid increase; the gradient was $\sim 60 \%$ of that observed in Stage I, but was still much larger than that for the low-concentration reaction and thus $\left[\mathrm{Pt}_{\mathrm{NC}}\right]$ increased rapidly again. From around 300 minutes, denoted as Stage IV, the slope of the area plot decreased slightly, with a gradient of about $1 / 3$ of that in the previous stage (Stage III), which was indicative of a slower increase in $\left[\mathrm{Pt}_{\mathrm{NC}}\right]$.

As shown in Figure 6-7, $L$ for the high-concentration reaction increased during Stage I from the initial $\sim 5 \mathrm{~nm}$ to $\sim 8.2 \mathrm{~nm}$ at 100 minutes. During Stage II, it remained almost constant between 100 and 180 minutes at $8.0 \pm 0.2 \mathrm{~nm}$. Interestingly during Stage III, 180-300 minutes, $L$ actually decreased from 8.1 to $7.6 \mathrm{~nm}$, differing markedly from the low-concentration reaction. After 300 minutes, $L$ continued to decrease gradually and was calculated to be $\sim 7.1 \mathrm{~nm}$ at the end of Stage IV. 


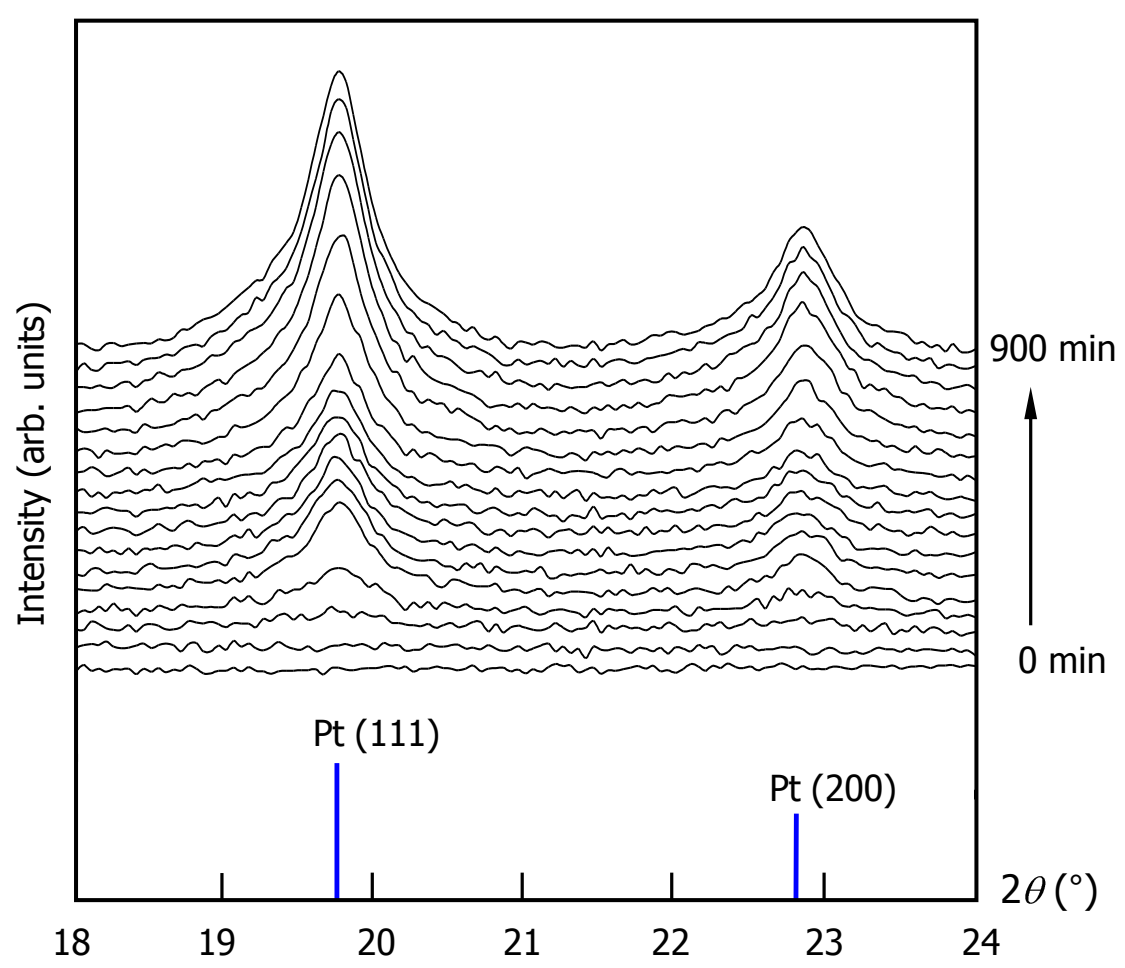

Figure 6-5. In situ XRD plot for high-concentration reaction.

Growth of $\mathrm{Pt}(111)$ and $\mathrm{Pt}(200)$ peaks over 900 minutes; the respective positions of the (111) and (200) reflections for bulk fcc platinum are shown by blue lines at the bottom of plot.

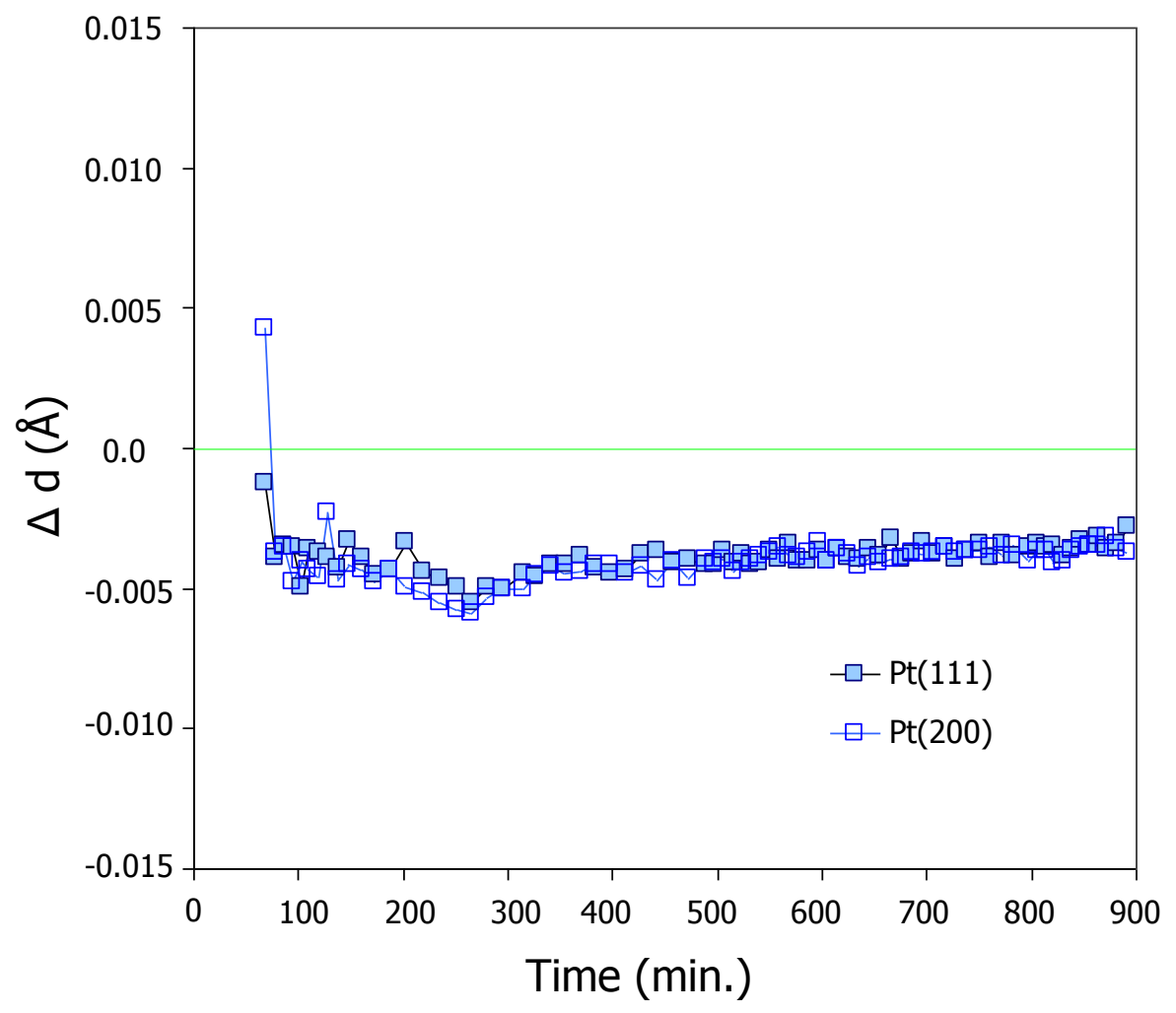

Figure 6-6. Shift in the calculated $d$-spacing from the bulk value for the high-concentration reaction.

$\Delta d=d_{\text {calculated }}-d_{\text {bulki }}$ the green line across at $\Delta d=0$ indicates $d=2.26$ and $1.96 \AA$, corresponding to bulk $\mathrm{Pt}(111)$ and $\mathrm{Pt}(200)$, respectively. 


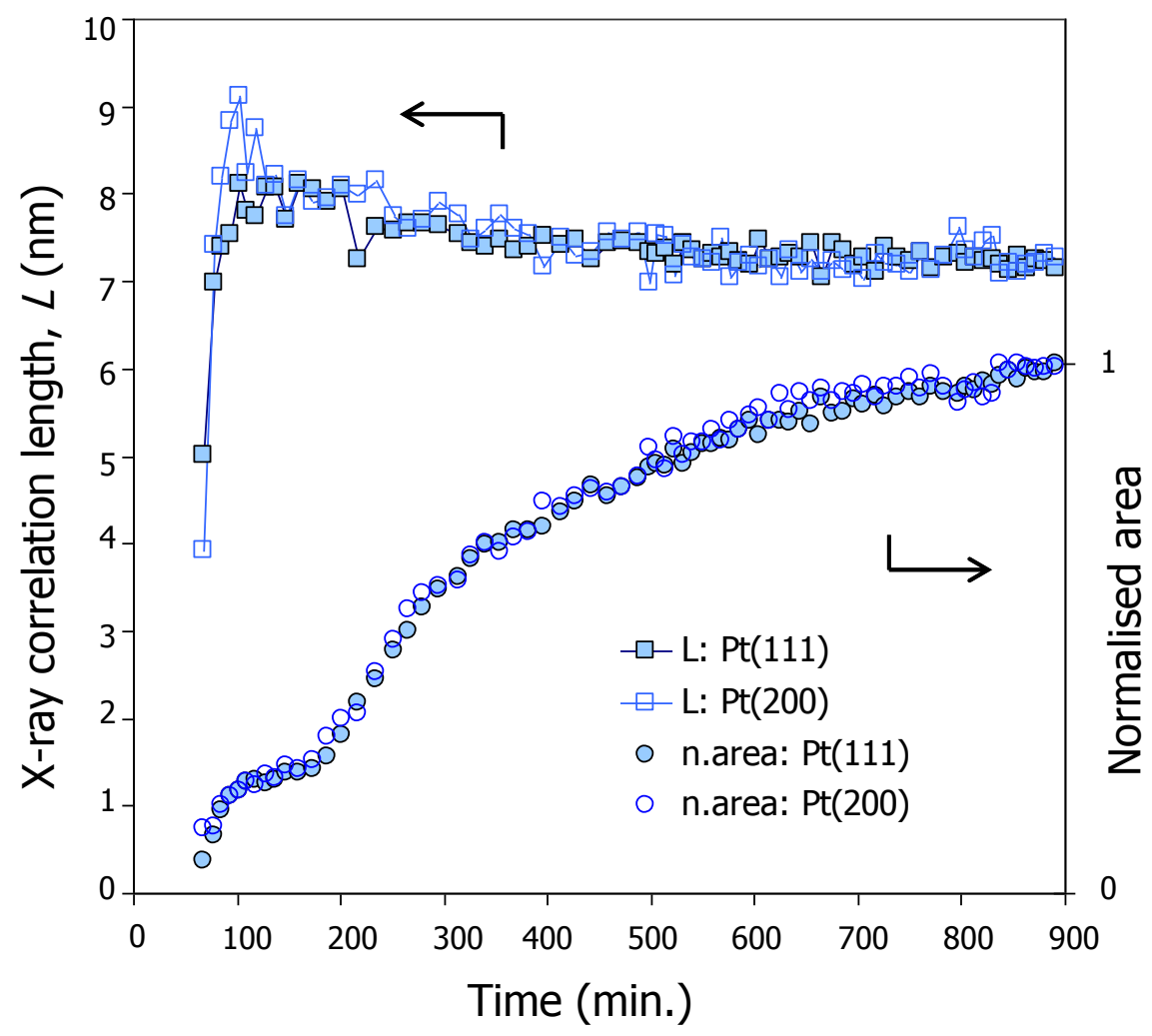

Figure 6-7. Normalised area and X-ray correlation length against time for the high-concentration reactions.

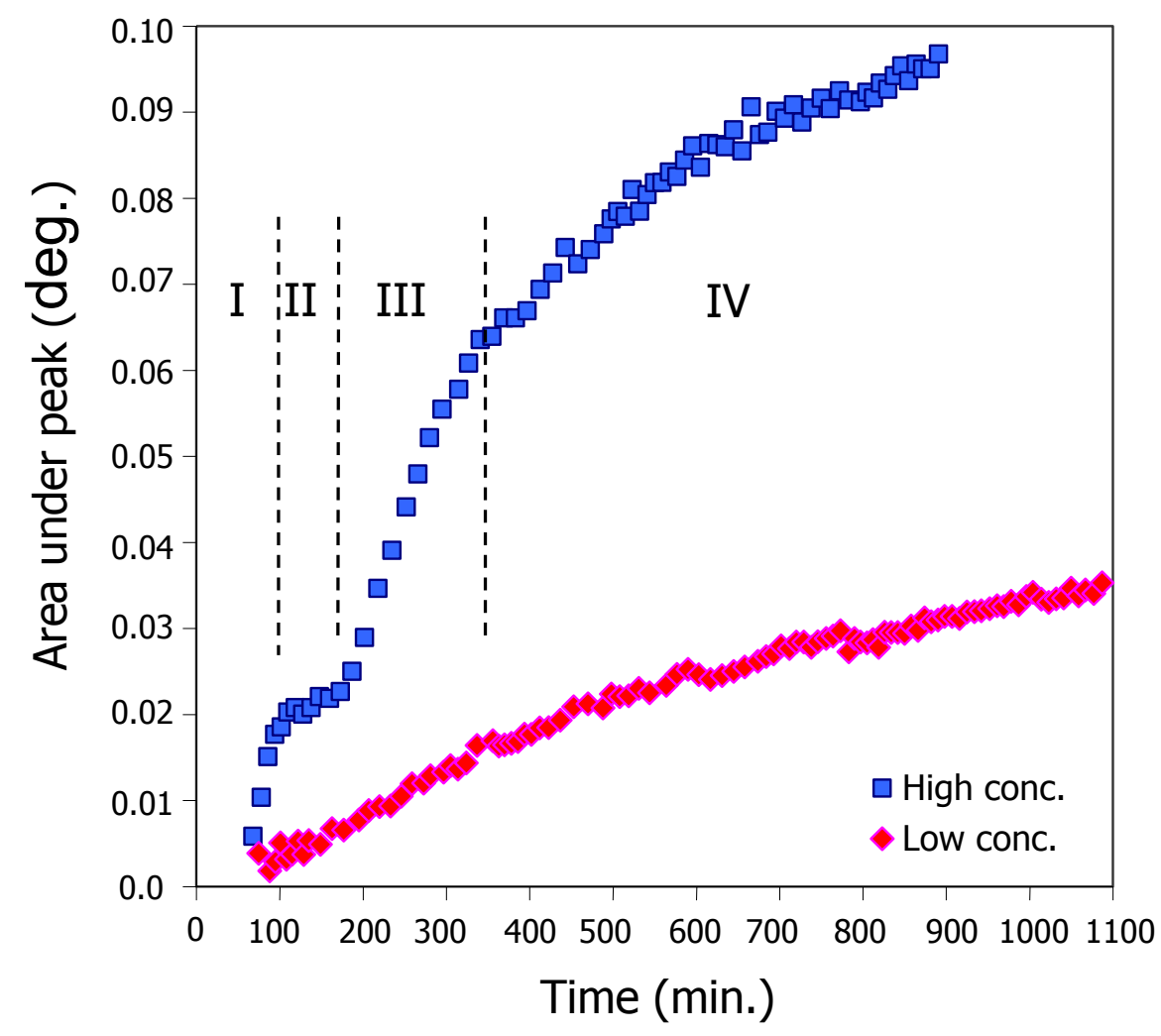

Figure 6-8. Time evolution of area under the $\mathrm{Pt}(111)$ peak.

Different growth rates of nanocrystalline platinum indicated by the evolution of area under the $\mathrm{Pt}(111)$ peak; I-V denote the growth stages of the high-concentration reaction. 
Figure 6-9 shows a TEM image of the platinum nanoparticles collected at the end of the in situ experiment of high precursor concentration. The nanoparticles were mainly of branched structures, and are described as multiply-branched nanoparticles. The average size of the nanoparticles was $50 \pm 9 \mathrm{~nm}$; where size was determined by measuring the longest distance across the particle.

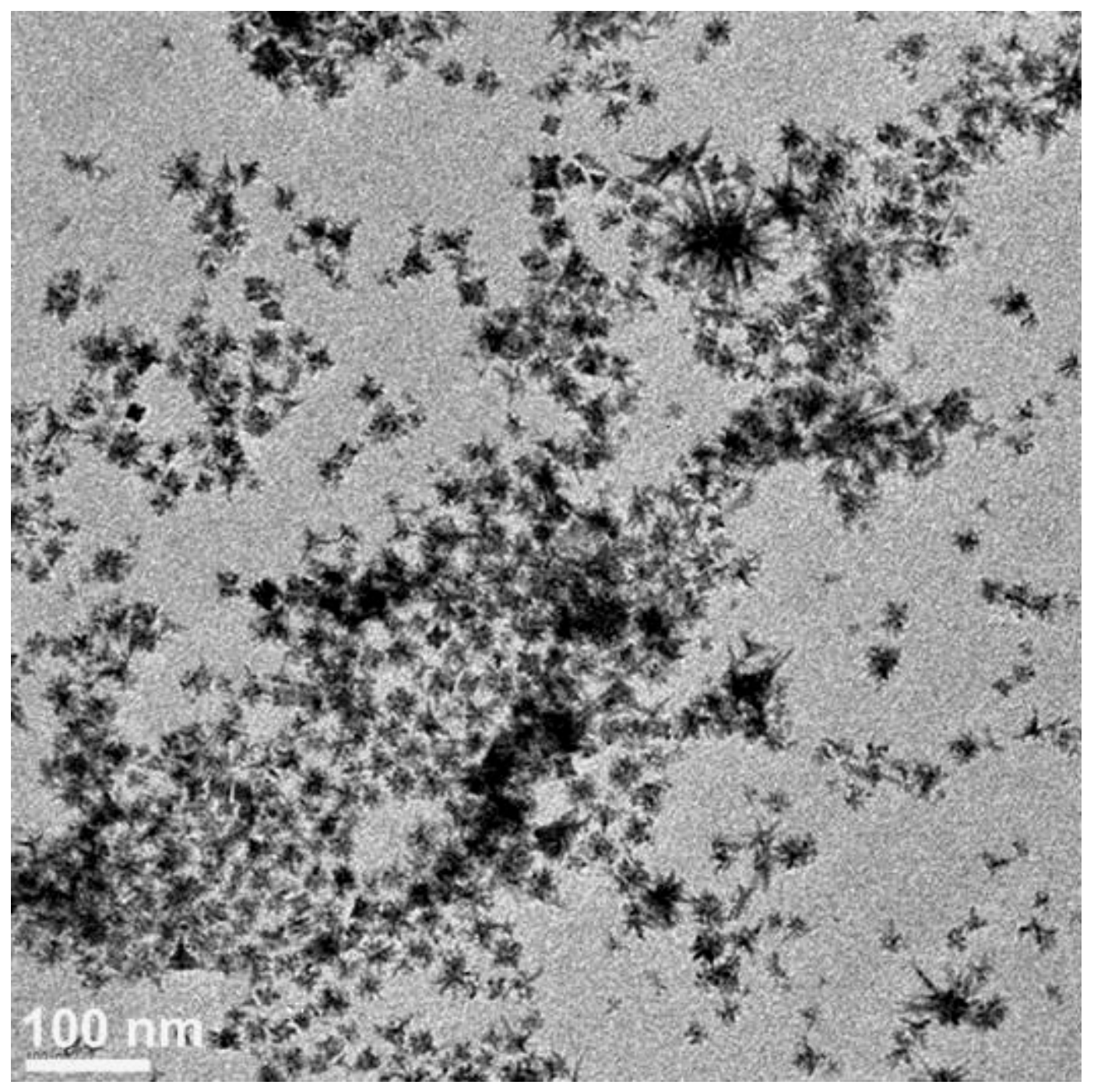

Figure 6-9. TEM image of nanoparticles collected at the end of the high-concentration in situ experiment. 


\subsubsection{Off-Line (Ex situ) Experiments}

To better understand the growth mechanisms of the low- and high-concentration reactions, a series of off-line (ex situ) experiments were carried out with each reaction quenched after certain growth stages and the product characterised using TEM. Due to the small volume of the reaction cell, intermediate products collected during early stages were too small in amount to be properly purified and characterised. Hence most of the offline experiments were carried out in a Fischer-Porter bottle. The reaction times for the off-line experiments were selected based on interesting regions in the XRD data. Experiments with different reaction conditions corresponding to the different growth stages are listed in Table 6-1. The purpose of Expts Pt-3C and Pt-4E was to compare the results of Fischer-Porter bottle reactions with those of the in situ reaction cell.

Table 6-1. Reaction conditions for off-line experiments.

\begin{tabular}{|c|c|c|c|c|}
\hline Expt & Concentration & $\begin{array}{c}\text { Reaction time } \\
\text { (min.) }\end{array}$ & Reaction vessel & Growth stage \\
\hline Pt-3A & Low & 80 & Fischer-Porter bottle & I \\
\hline Pt-3B & Low & 480 & Fischer-Porter bottle & II \\
\hline Pt-3C & Low & 1080 & Fischer-Porter bottle & II \\
\hline Pt-4A & High & 75 & Fischer-Porter bottle & II \\
\hline Pt-4B & High & 120 & Fischer-Porter bottle & III \\
\hline Pt-4C & High & 240 & Reaction cell & IV \\
\hline Pt-4D & High & 500 & Reaction cell & IV \\
\hline Pt-4E & High & 900 & Fischer-Porter bottle & \\
\hline
\end{tabular}

\section{Expt Pt-3A. Low-concentration reaction at 80 minutes}

As shown in Figure 6-10, the purified product collected from a reaction quenched at 80 minutes comprised faceted nanoparticles of irregular shapes, with nanoparticle size measured in the range of $6 \pm 2 \mathrm{~nm}$. HRTEM imaging showed that some of the nanoparticles were twinned while most were single crystals, with a 1:6 ratio of twinned to single crystal. As these nanocrystals were later observed to be the building blocks for larger nanocrystals, they are described as primary crystallites. 


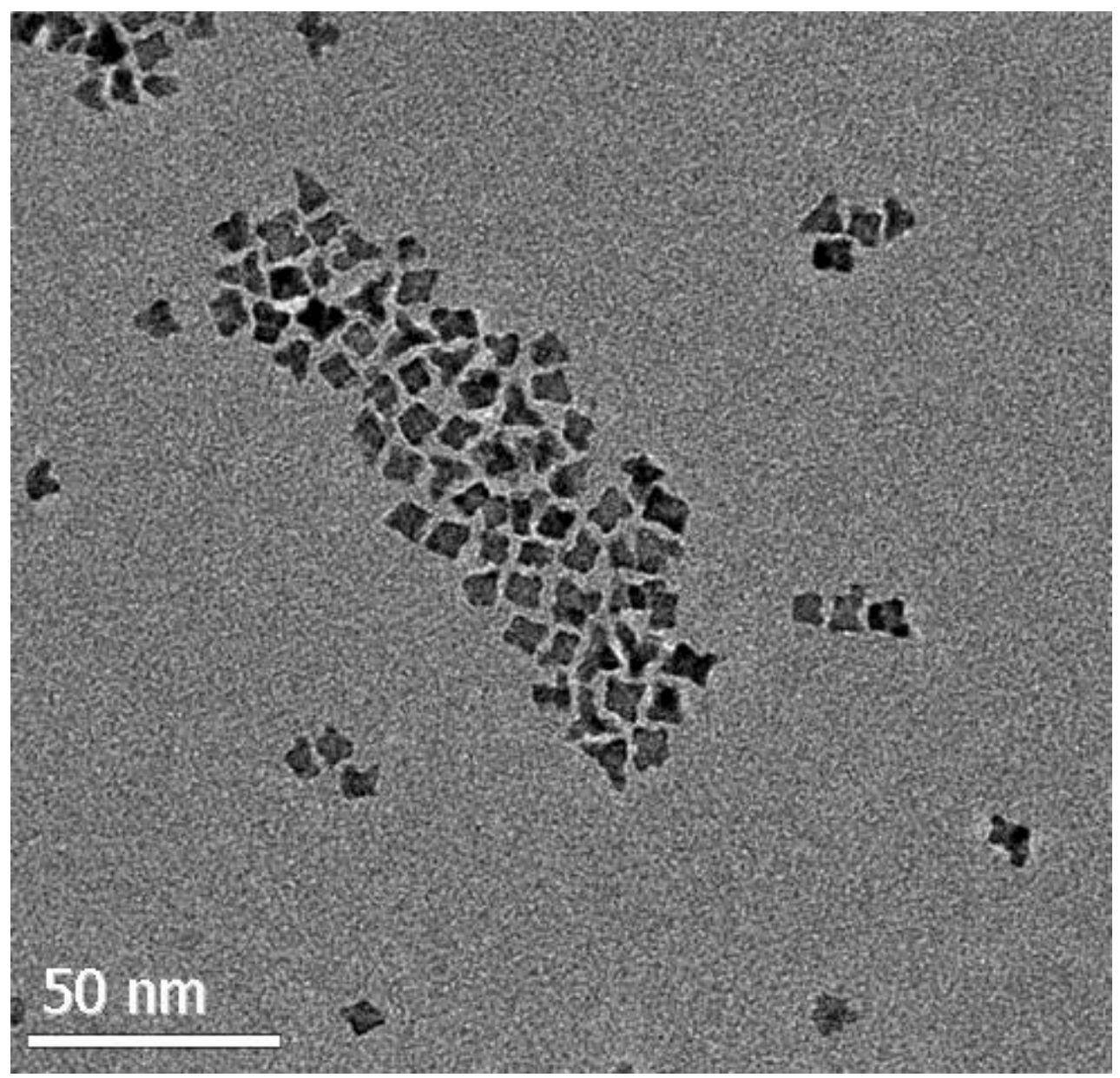

Figure 6-10. TEM image of platinum nanoparticles from low-concentration reaction at 80 minutes (Pt-3A).

\section{Expt Pt-3B. Low-concentration reaction at 480 minutes}

As shown in Figure 6-11a, the nanoparticles collected at 480 minutes, in the middle of Stage II, adopted an interesting morphology that is somewhat different from those observed in Expts Pt-1 and Pt-3A. Close examination showed that each individual nanoparticle, averaging at $13 \pm 2 \mathrm{~nm}$, was made up of at least 4 sub-units of $5 \pm 1 \mathrm{~nm}$ attached together. These nanoparticles are thus described as attached nanocrystals. HRTEM image of a typical attached nanocrystal is shown in Figure 6-11b, with the corresponding power spectrum shown in Figure 6-11c. The nanocrystal was observed to consist of 4 cube-like structures - the sub-units, which were of darker contrast. The regions between the sub-units, which were of lighter contrast, indicated thinner cross sections within the attached nanocrystal. The whole attached nanocrystal was observed to be projected along a $\langle 100\rangle$ zone axis and hence all the sub-units must be in the same crystallographic orientation. 


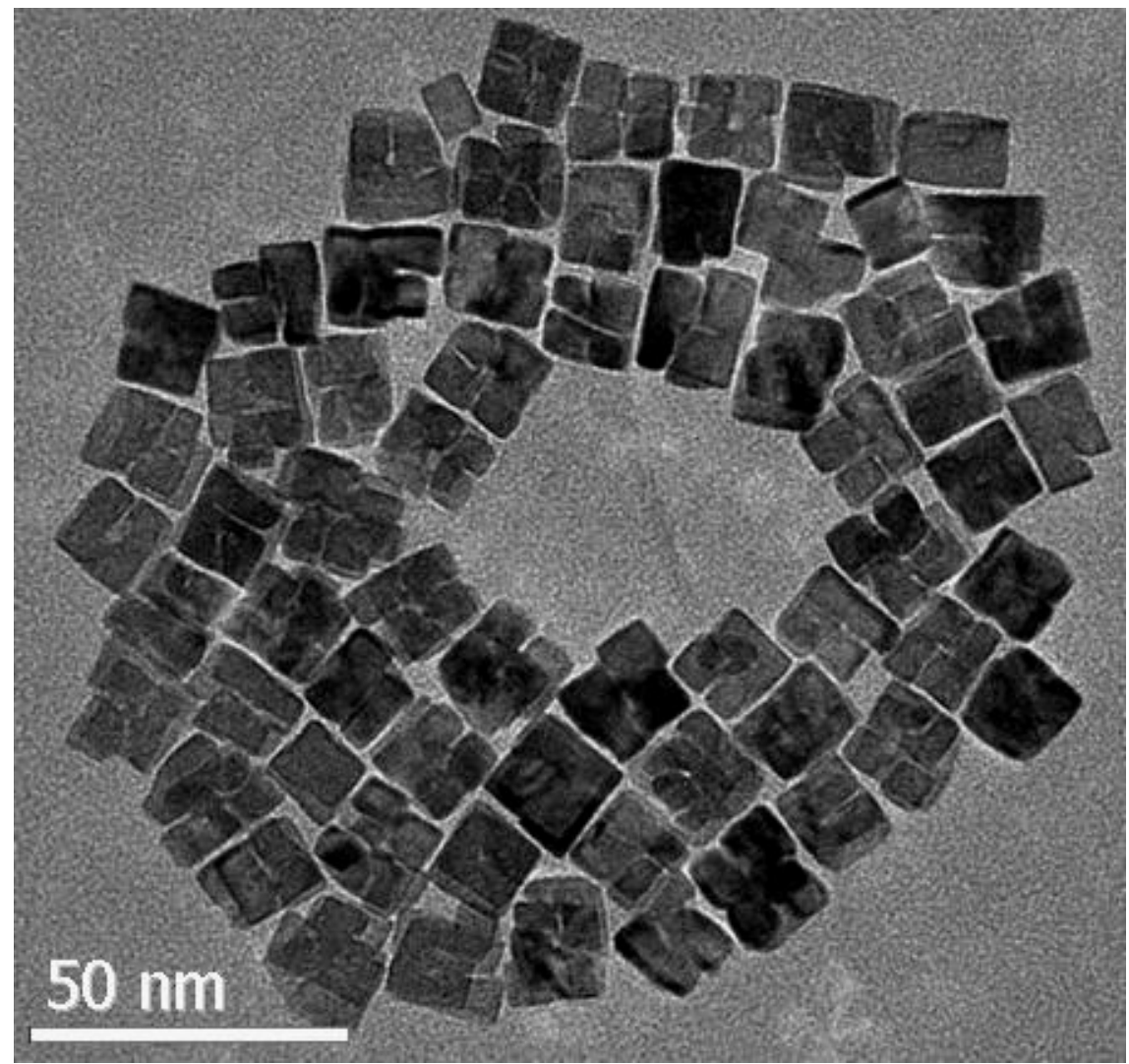

(a)
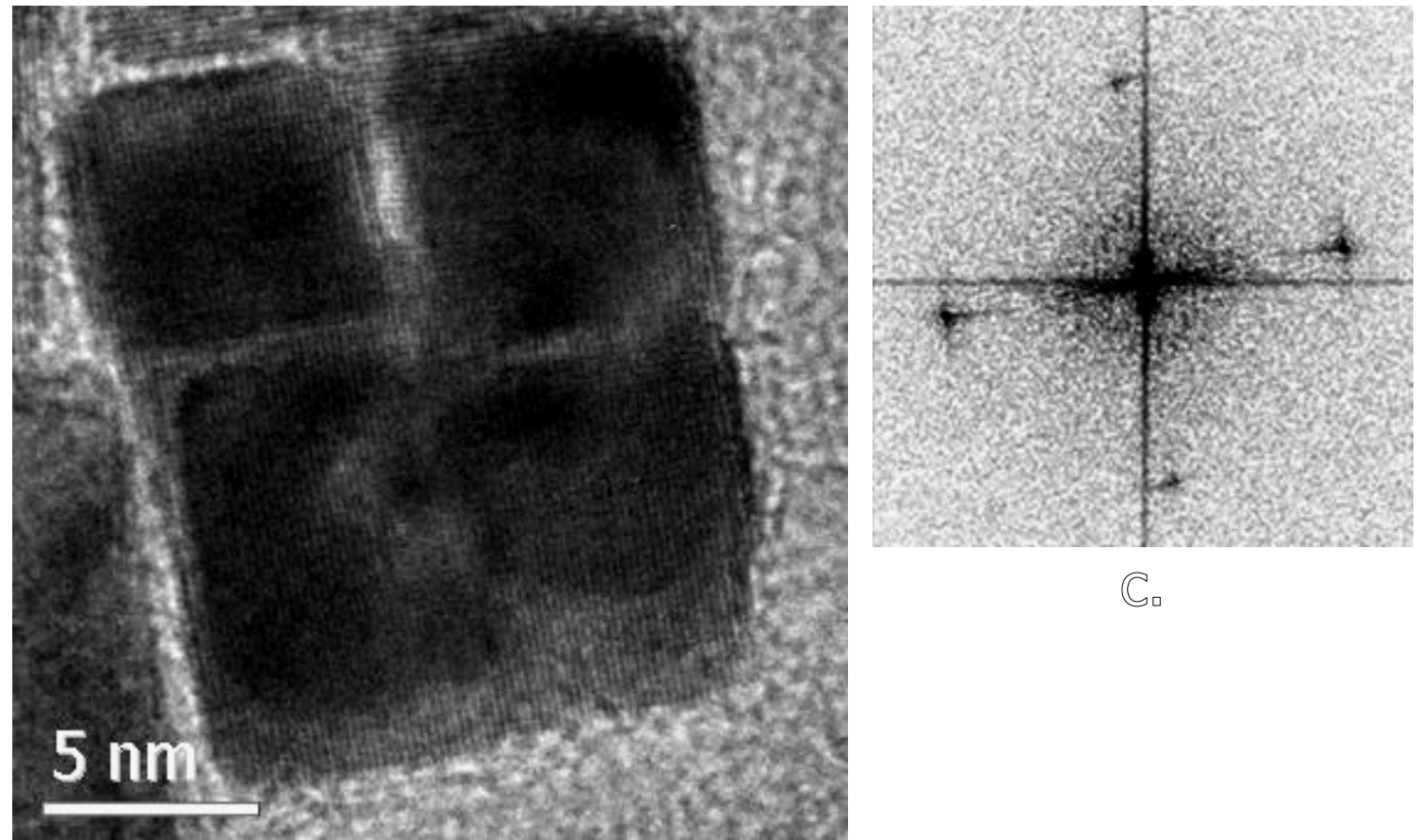

Co

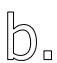

Figure 6-11. TEM images of attached nanocrystals from low-concentration reaction at 480 minutes (Pt-3B).

a. TEM image of the nanoparticles collected. b. HRTEM image of a typical attached nanocrystal. c. Power spectrum of image $b$, which can be matched to a $\langle 100\rangle$ projection of the fcc structure. The nanocrystal is shown to be a single crystal Pt being projected along a $\langle 100\rangle$ zone axis. 


\section{Expt Pt-3C. Low-concentration reaction at 1080 minutes}

The synthesis in Expt Pt-3C was a repetition of Expt Pt-1, but in a Fischer-Porter bottle. TEM characterisation showed that the nanoparticles collected from Expt Pt-3C were the same as those observed in Expt Pt-1. Figure 6-12, a HRTEM image shows that these nanoparticles adopted similar morphologies to those observed in Figure 6-10 (Expt Pt-3A, at 80 minutes), but were of bigger size range, averaging at $13 \pm 2 \mathrm{~nm}$.

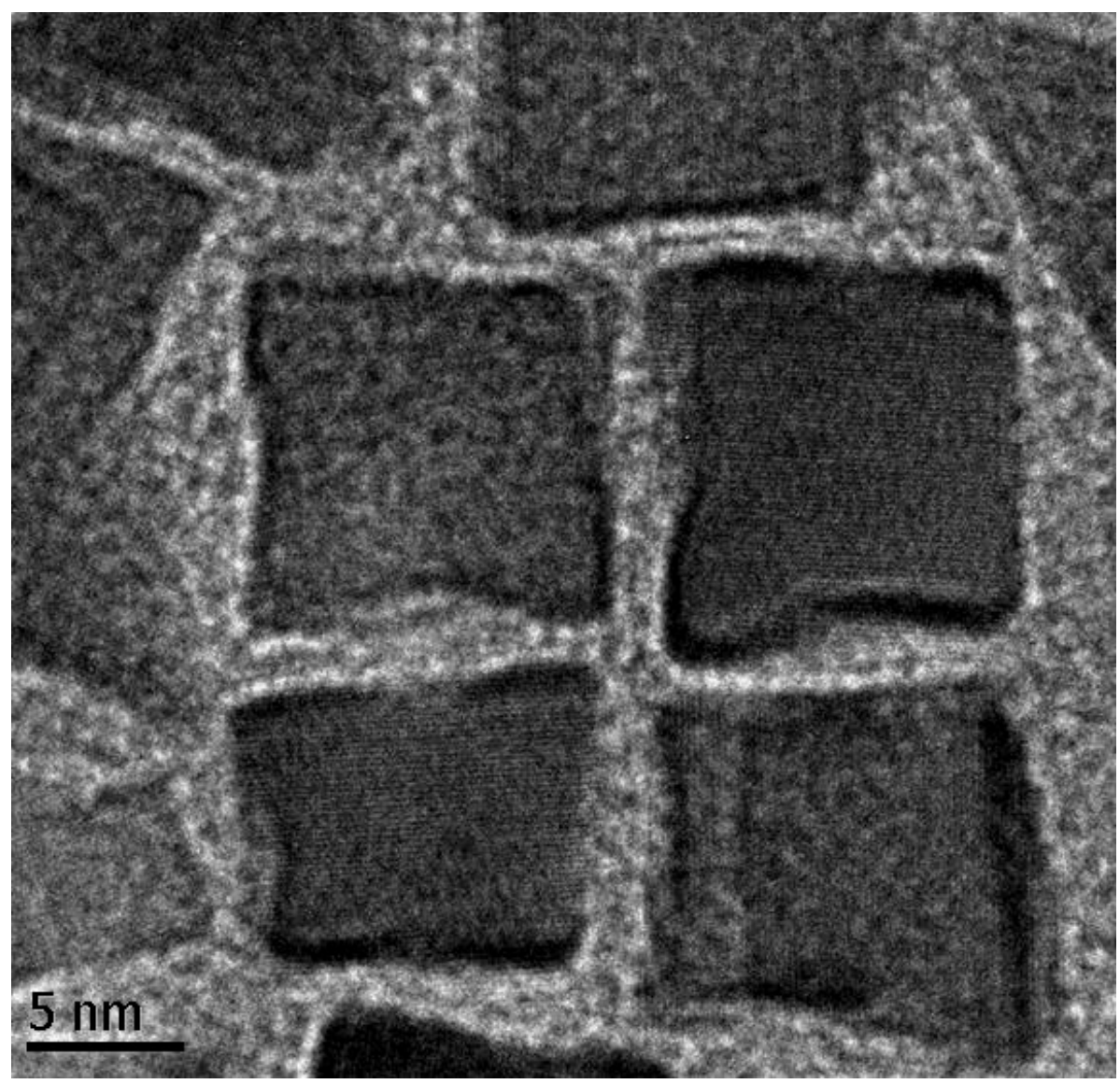

Figure 6-12. TEM image of the platinum nanoparticles from low-concentration reaction at 1080 minutes (Pt-3C).

\section{Expt Pt-4A. High-concentration reaction at 75 minutes}

Figure 6-13a shows the TEM image of the platinum nanoparticles obtained at 75 minutes of a high-concentration reaction, the middle of Stage I. These nanoparticles adopted a nearly cubic morphology with an average size of $6.5 \pm 0.6 \mathrm{~nm}$. 

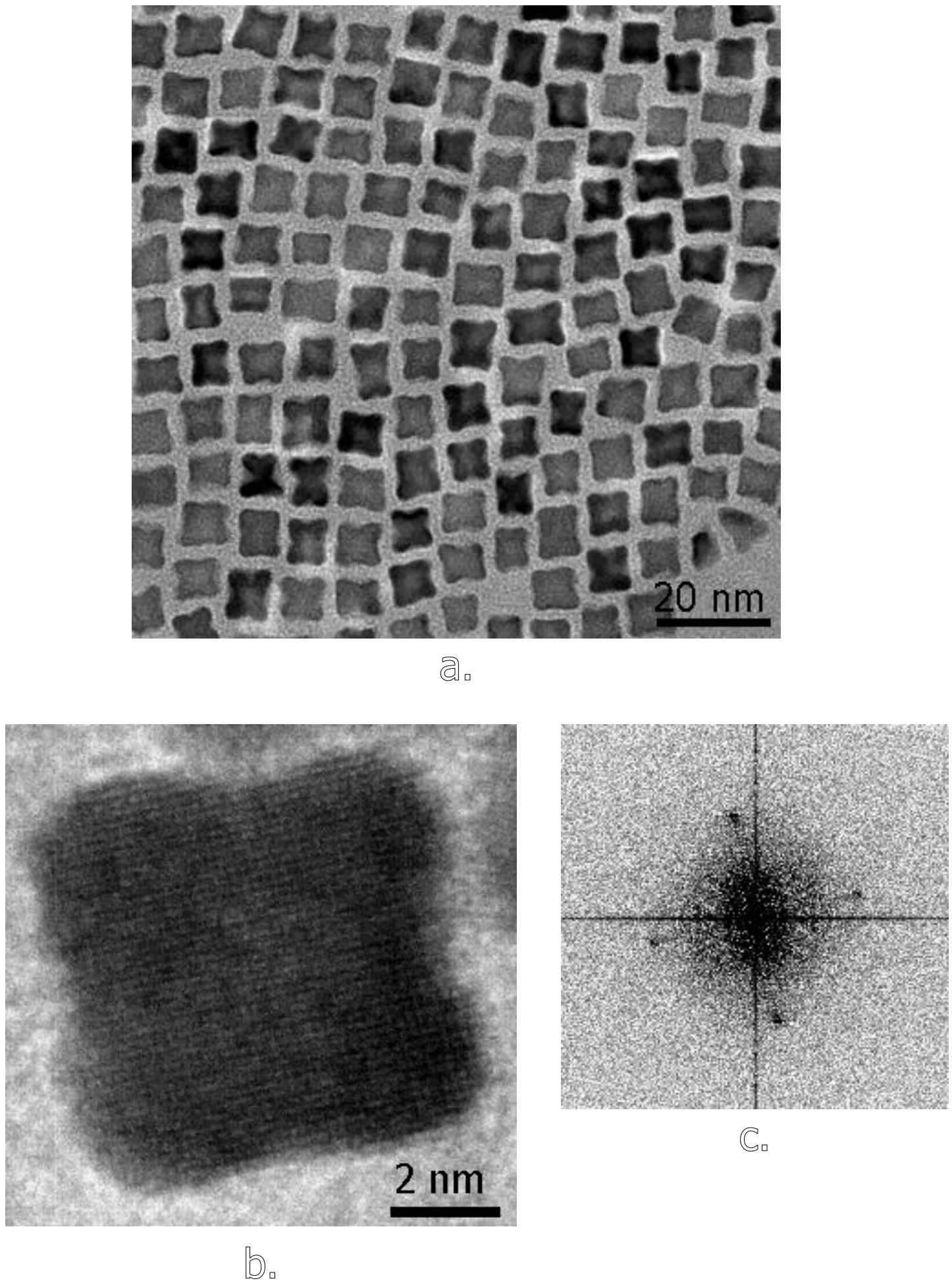

Co

Figure 6-13. TEM images of quasi-octapods from high-concentration reaction at 75 minutes (Pt-4A).

a. TEM image of the nanoparticles obtained, which had assembled into a monolayer of cubic packing. b. HRTEM image of a typical quasi-octapod which shows single crystal structure.

c. Power spectrum of image $b$ which can be matched to $a\langle 100\rangle$ projection of fcc structure. 
An HRTEM image of a typical nanocrystal is shown in Figure 6-13b, with the corresponding power spectrum shown in Figure 6-13c. The platinum nanoparticle was observed to be a single crystal being projected along a $\langle 100\rangle$ zone axis. On the basis of this projection, the exposed flat faces of the nanocrystal could be identified as the $\{100\}$ facets. However, differing from a nanocube that has sharp corners/vertices, the nanocrystal had protruded and rounded corners appearing as short arms projecting along the $\langle 111\rangle$ directions. Generally nanoparticles with eight arms/pods are known as octapods, but because of the short arms displayed by the nanocrystals observed here, they are described as quasi-octapods.

\section{Expt Pt-4B. High-concentration reaction 120 minutes}

Figure 6-14a shows the platinum nanoparticles collected at 120 minutes, the middle of Stage II. These nanoparticles were larger in size, averaging at $11.2 \pm 0.6 \mathrm{~nm}$, and have the same $\langle 100\rangle$ orientation as displayed by the quasi-octapods. Figure 6-14b shows the HRTEM image of a typical nanocrystal obtained during this stage, with the corresponding power spectrum shown in Figure 6-14c, indicating again a single-crystalline structure and a $\langle 100\rangle$ orientation. Close examination on the HRTEM images revealed that these nanocrystals did not appear exactly the same as the quasi-octapods. On the basis of observations in the TEM, the nanocrystals at 120 minutes had thinner cross-sections towards the centre of the $\{100\}$ faces, which is indicative of etching. The arms of the quasi-octapods had grown by $\sim 2.5 \mathrm{~nm}$ along the $\langle 111\rangle$ directions, while the cross-section towards the centre of the $\{100\}$ facets had reduced by 1-2 $\mathrm{nm}$. These nanocrystals are thus described as etched-octapods. Both the quasi- and the etched-octapods tend to assemble into cubic packing when the nanoparticle suspension dries on a TEM grid. 

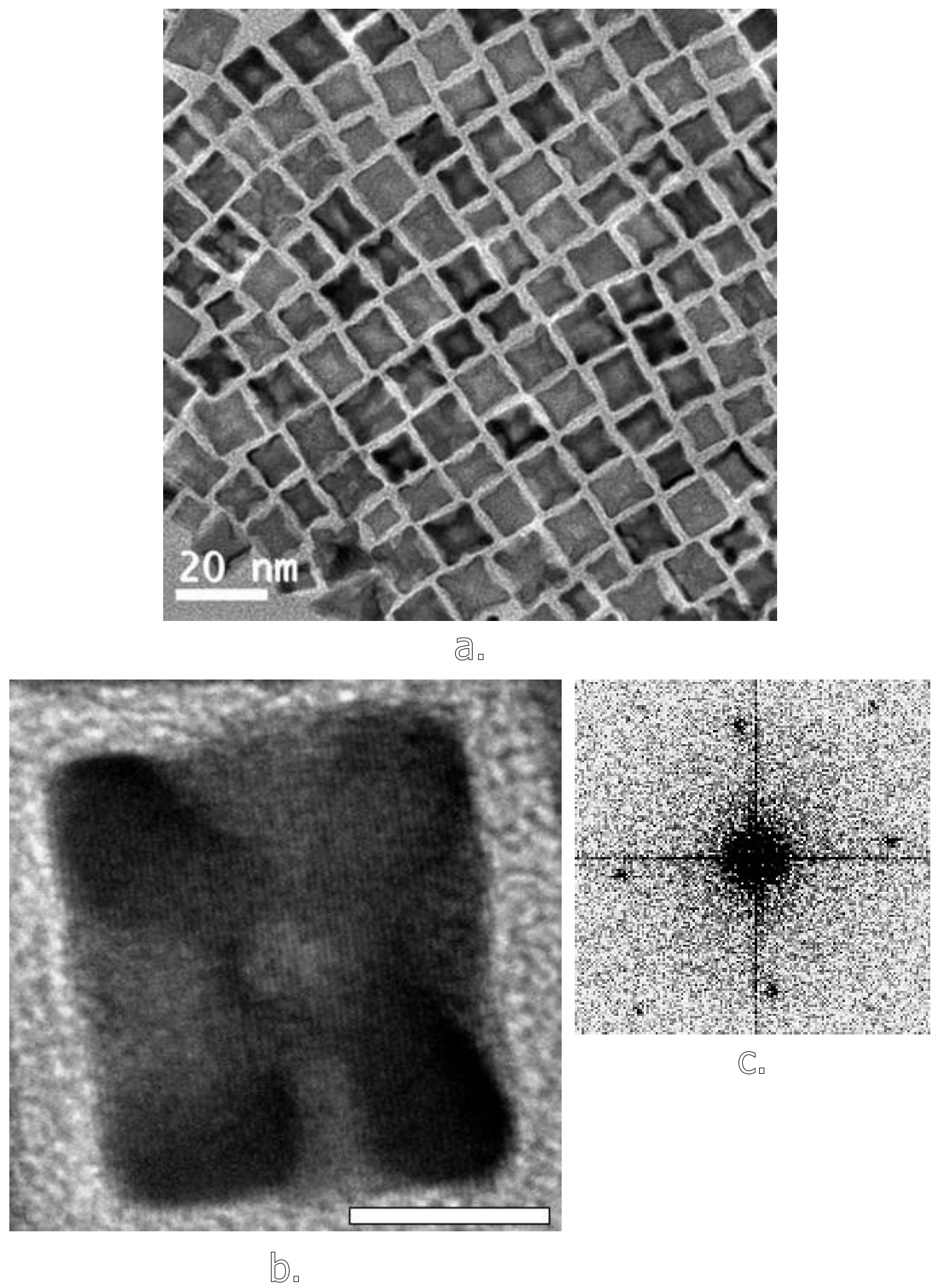

Figure 6-14. TEM images of etched-octapods from high-concentration reaction at 120 minutes (Pt-4B).

a. TEM image showing cubic packing assembly of the nanoparticles obtained. b. HRTEM image of a typical etched octapod, which is of single crystal structure. c. Power spectrum of image b, which matches a $\langle 100\rangle$ projection of the fcc structure. 


\section{Expt Pt-4C. High-concentration reaction at 240 minutes}

Figure 6-15a shows the platinum nanocrystals obtained at 240 minutes, the middle of Stage III. The nanocrystals comprised a mixture of etched-octapods and porous nanostructures, in a ratio of $\sim 1: 3$. The latter is also known as porous platinum ${ }^{[17]}$ and is described as porous nanocrystals. A typical porous nanocrystal is shown in Figure 6-15b, with the power spectrum of the image of the whole particle shown in Figure 6-15c. The nanocrystal may appear to be consisting of a number of attached crystallites, but it was observed to be a single structure, with a projection along a $\langle 100\rangle$ direction. The porous nanocrystals were typically $19 \pm 2 \mathrm{~nm}$ in size.

\section{Expt Pt-4D. High-concentration reaction at 500 minutes}

As shown in Figure 6-16a, the platinum nanocrystals obtained at 500 minutes, i.e. middle of Stage IV, were almost exclusively porous nanocrystals. In Figure 6-16b, the HRTEM image of a typical porous nanocrystal shows clearly the lattice fringes across the entire nanocrystal indicating single crystallinity of the nanocrystal. The power spectrum of the image, as shown in Figure 6-16c, matched a $\langle 110\rangle$ projection of the fcc structure. With an average size of $24 \pm 3 \mathrm{~nm}$, these porous nanocrystals were generally larger than the same species obtained at 240 minutes. 


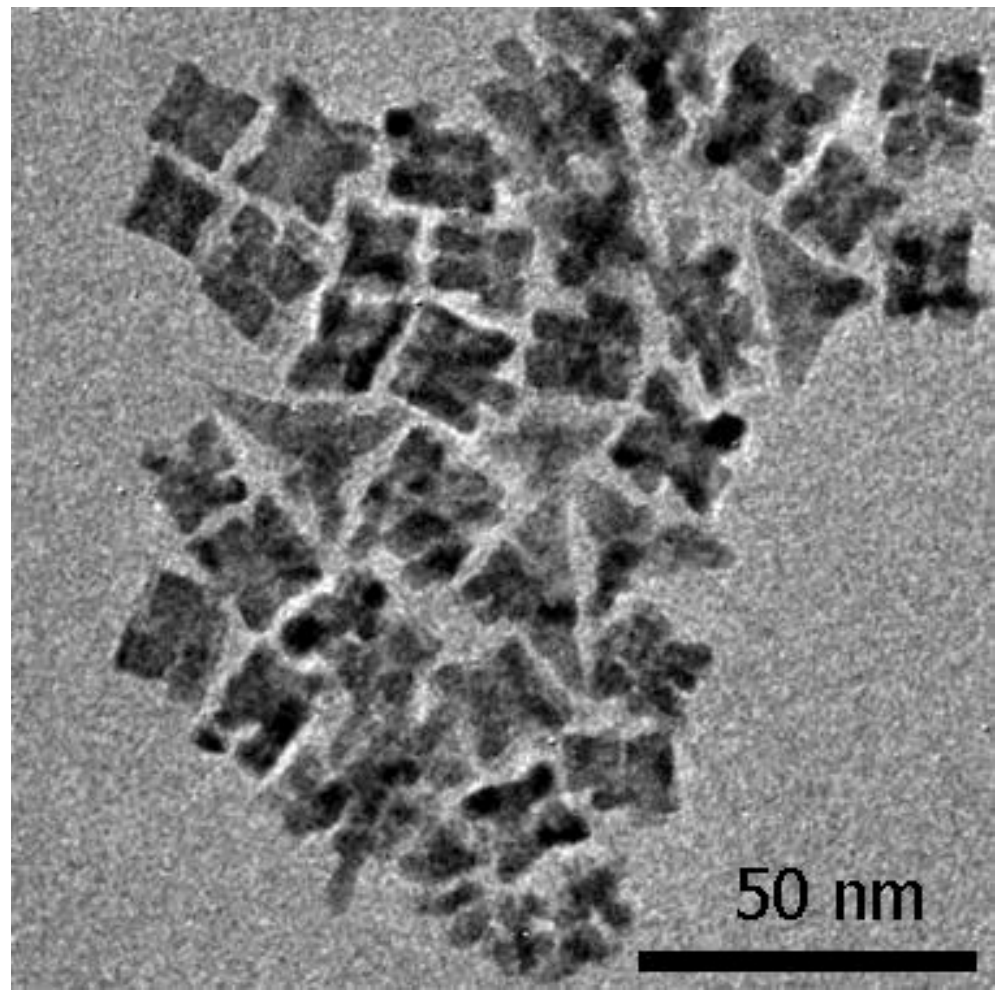

(.).
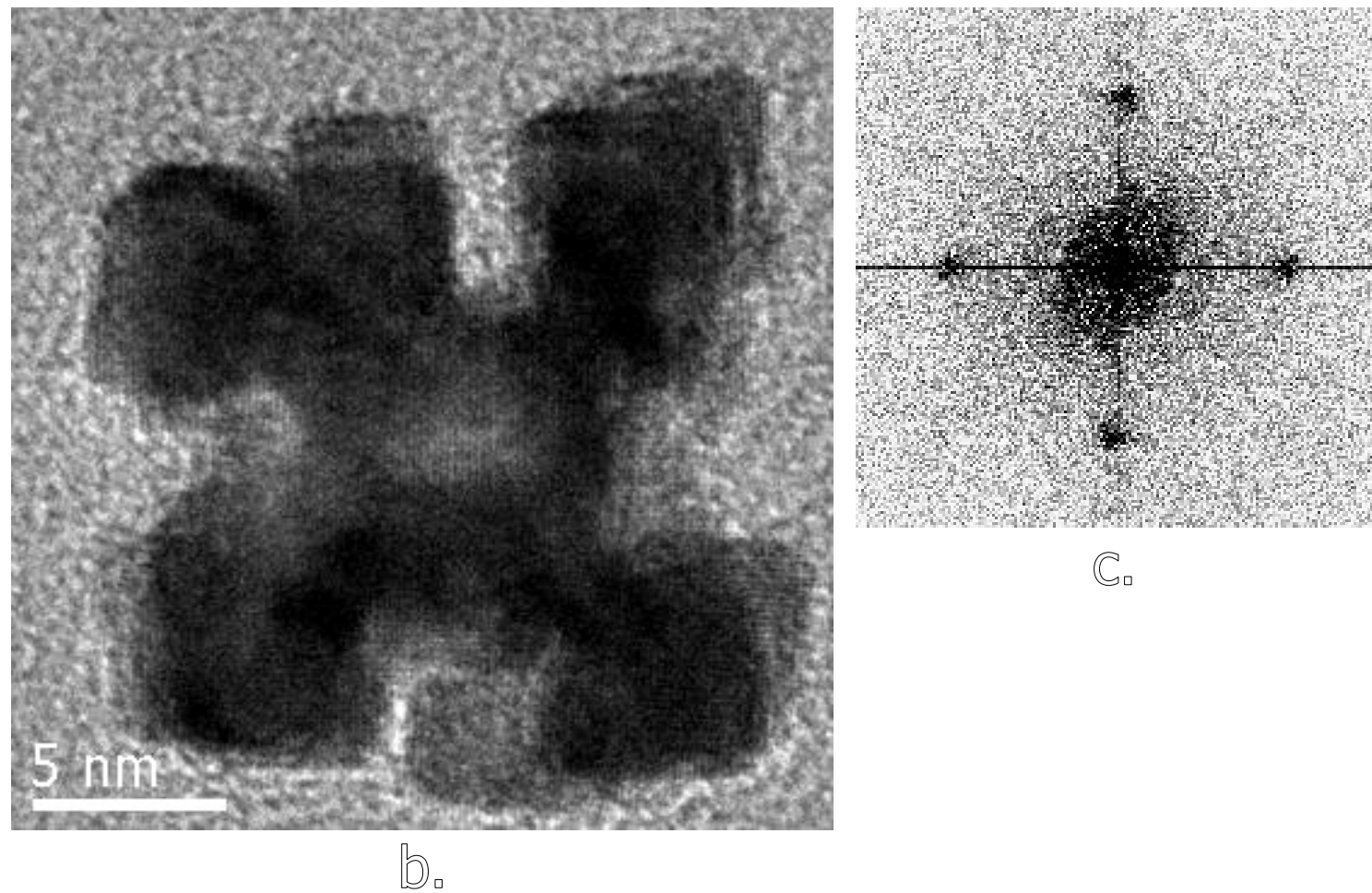

Figure 6-15. TEM images of porous nanocrystals from high-concentration reaction at 240 minutes (Pt-4C).

a. TEM image of the nanoparticles obtained. b. HRTEM image of a typical porous nanocrystal in Stage III of the growth. c. Power spectrum of image b, matching a $\langle 100\rangle$ projection of fcc Pt. 


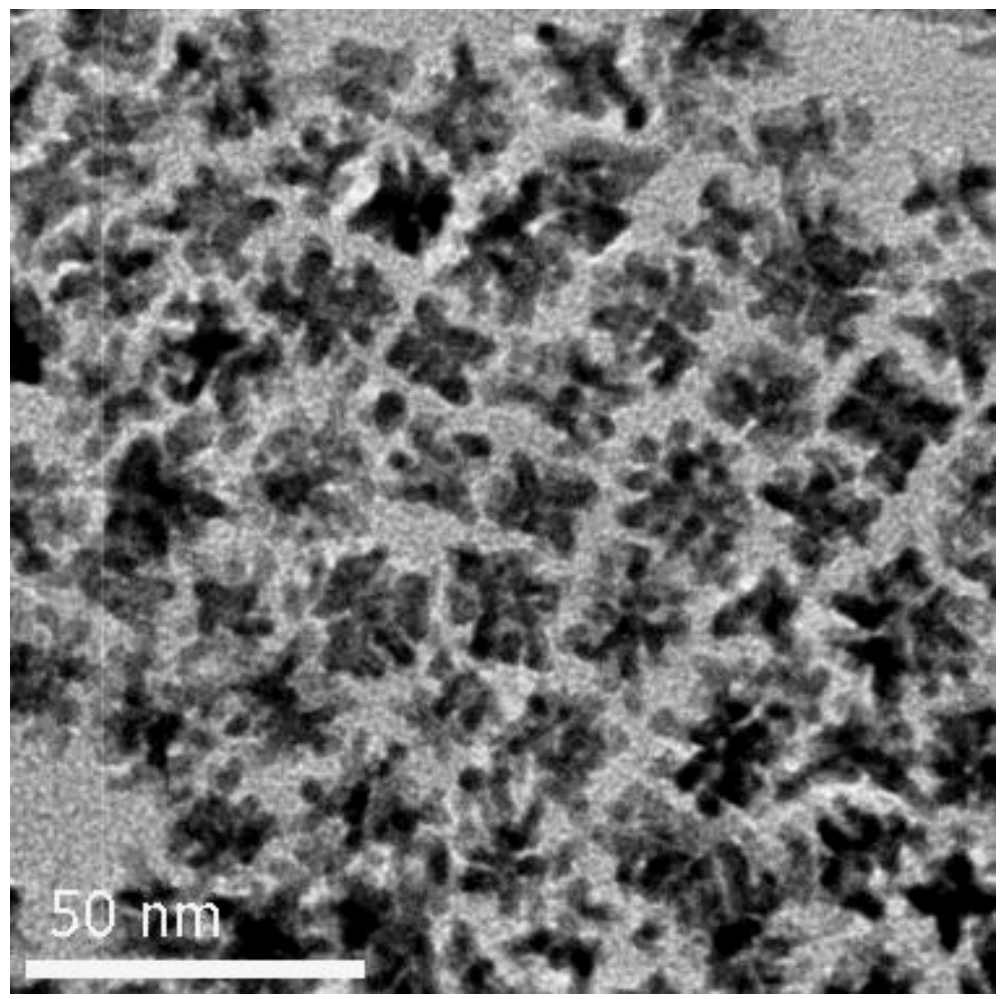

(a)
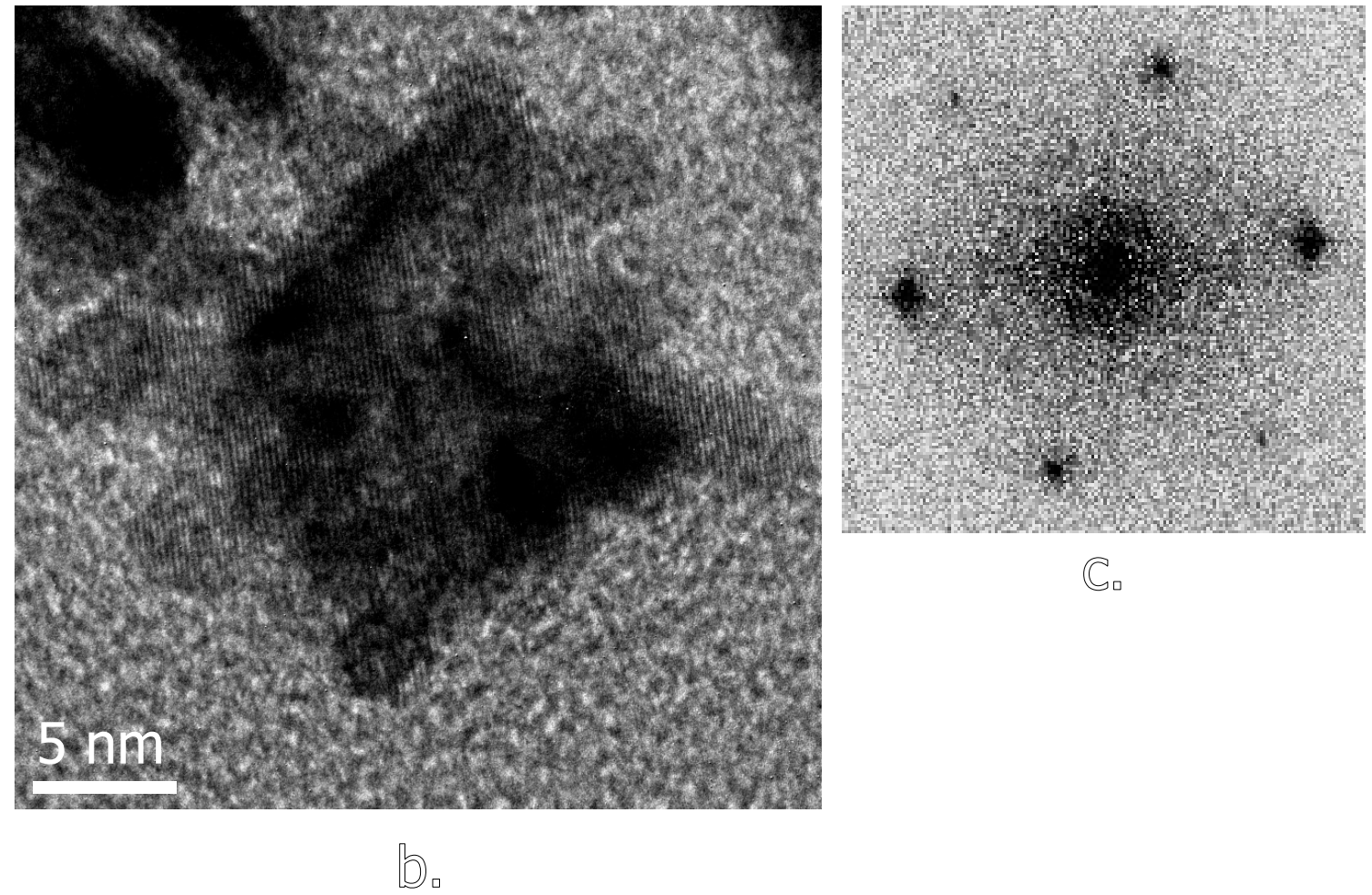

Figure 6-16. TEM images of porous nanocrystals from high-concentration reaction at 500 minutes (Pt-4D).

a. TEM image of the nanoparticles obtained. b. HRTEM image of a typical porous nanocrystal in Stage IV of the growth. $\mathbf{c}$. Power spectrum of image $b$ indicating single crystal structure and $a$ $\langle 110\rangle$ projection of the platinum nanocrystal. 


\section{Expt Pt-4E. High-concentration reaction at 900 minutes}

The synthesis in Expt Pt-4E was a repetition of Expt Pt-2, but was carried out in a Fischer-Porter bottle. TEM characterisation showed that the nanoparticles collected from Expt Pt-4E were the same as the multiply-branched nanoparticles observed in Expt Pt-2. Observations in the TEM showed that the multiply-branched nanoparticles were of polycrystalline structures.

Figure 6-17a shows the TEM image of a typical multiply-branched nanoparticle obtained from Expt Pt-4E. As can be seen, the nanoparticle comprised multiple branches extending outward from the centre of the particle. Each branch originated from the centre terminated with a secondary branch or multiple branching. Likewise, further branch formation or branching appeared in a similar manner for the secondary branches and branches of higher orders. These branches were 2-3 nm in diameter when measured on TEM images. Each branch or sub-branch was of a different crystal orientation, giving rise to a highly polycrystalline structure. Figures $6-17 \mathbf{b}$ and $\mathbf{c}$ show the HRTEM image of part of the nanoparticle and the corresponding power spectrum, respectively. 


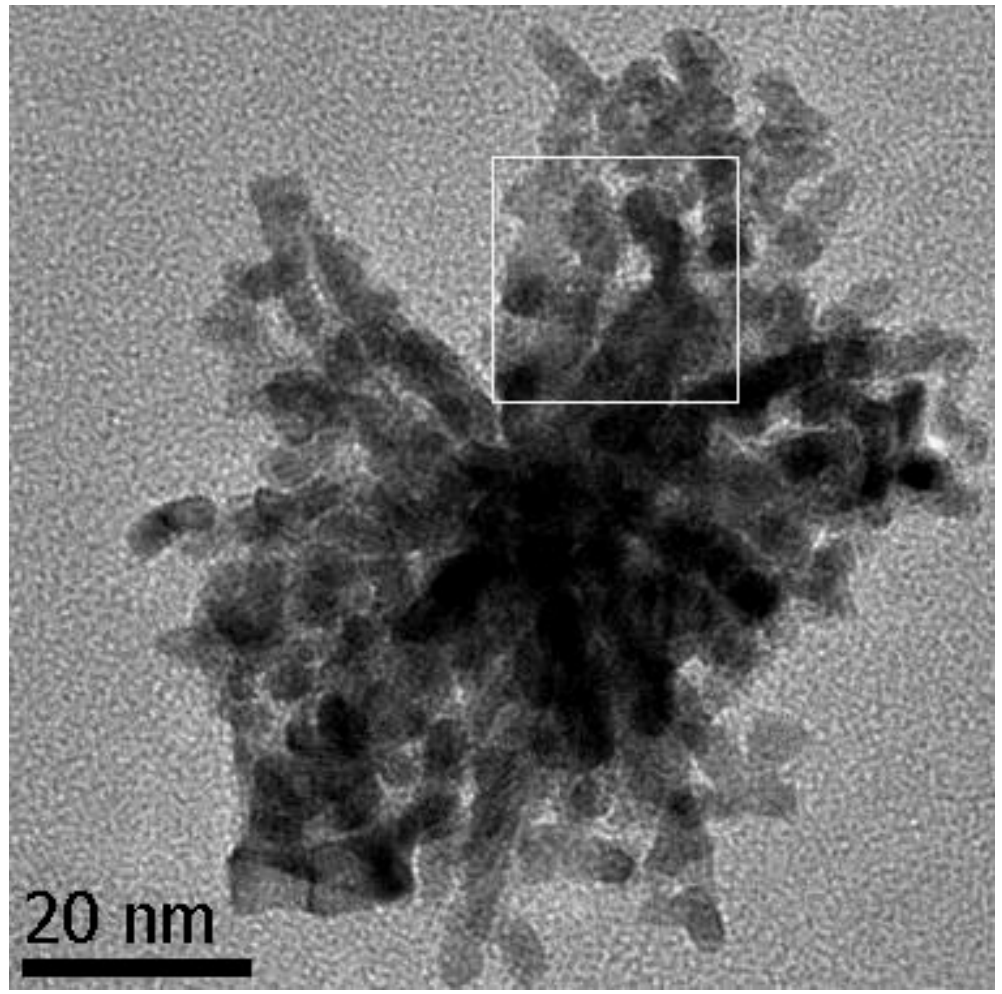

()
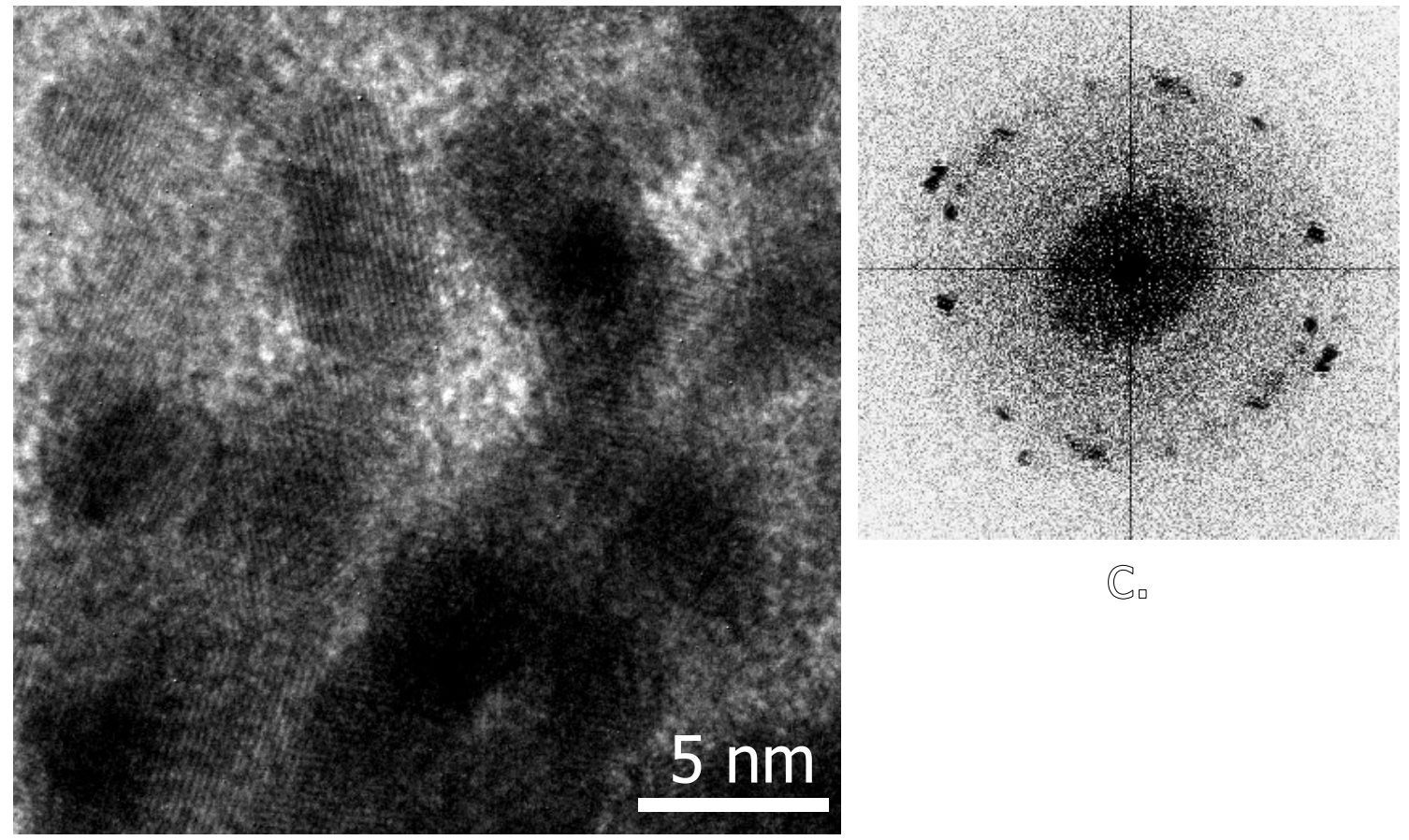

(1)

Figure 6-17. TEM image of multiply-branched nanoparticle from high-concentration reaction at 900 minutes (Pt-4E).

a. TEM image of a typical multiply-branched nanoparticle. b. HRTEM image of part of the nanoparticle (indicated by square in image a), showing the polycrystalline nature of the nanoparticle. c. Power spectrum of image $b$. 


\subsection{Discussion}

\subsubsection{Growth Mechanism: Low-Concentration Reaction}

The growth of platinum nanocrystals in the low-concentration reaction occurs in Stages I and II, as indicated by the change in the X-ray correlation length $(L)$ in Figure 6-3. The most interesting observation for the low-concentration reaction was at about 250 minutes. At this point in time, the average crystallite size as indicated by $L$, reached the average maximum value; whereas the amount of crystallites present was just slightly more than $30 \%$ of the total crystallites collected at the end of the in situ experiment. $L$ was then observed to remain constant while the nanocrystalline platinum concentration $\left(\left[\mathrm{Pt}_{\mathrm{NC}}\right]\right)$ continued to increase. TEM observations of the nanoparticles at different stages suggest the growth involves the attached nanocrystals shown in Figure 6-11.

\section{Stage I. Formation and attachment of primary crystallites}

From the XRD data, the $\left[\mathrm{Pt}_{\mathrm{NC}}\right]$ in Stage I increased approximately linearly with time, whereas $L$ (an indication of average crystallite size) was shown to increase relatively rapidly from 3 to $8.5 \mathrm{~nm}$. TEM evidence indicated the formation of mainly single crystalline structures of faceted and non-branched morphologies during this stage. The average nanoparticle size of $5 \mathrm{~nm}$ was closer to that indicated by the XRD data in the middle of Stage I. It is believed that the primary crystallites were mostly formed at around 100 minutes of the experiment. These nanocrystals then began to orientate among each other and attach with each other in groups of 4-10 nanocrystals. As the primary crystallites began to attach together in an oriented fashion, the average crystallite size, and hence $L$, increased considerably.

\section{Stage II. Growth of attached nanocrystals}

In Stage II, the $\left[\mathrm{Pt}_{\mathrm{NC}}\right]$ was indicated to be growing continuously while the average crystallite size remained approximately constant. TEM results suggested that most of the primary crystallites formed in Stage I had begun to attach together in groups of 4-10 crystallites in early Stage II. 
Consider the attachment of two iso-oriented crystallites. Free platinum atoms adsorb onto the two crystal planes closely facing each other and eventually fuse the two crystallites. As complete fusion takes time to achieve, there exists regions or gaps at the interface between the crystallites. These gaps were observed as the lower contrast regions in the TEM image (Figure 6-11b). The amount of crystalline platinum present thus increases as more gaps are filled by the free platinum atoms from solution. As $L$ was an indication of the average crystallite size, its increase was expected to be less obvious between one stage in which fusion had begun, and the other stage in which fusion was near completion. Besides, it is also possible that there was small amount of primary crystallites present in the solution and did not participate in any attachment process. The proposed growth mechanism is illustrated in the scheme in Figure 6-18.

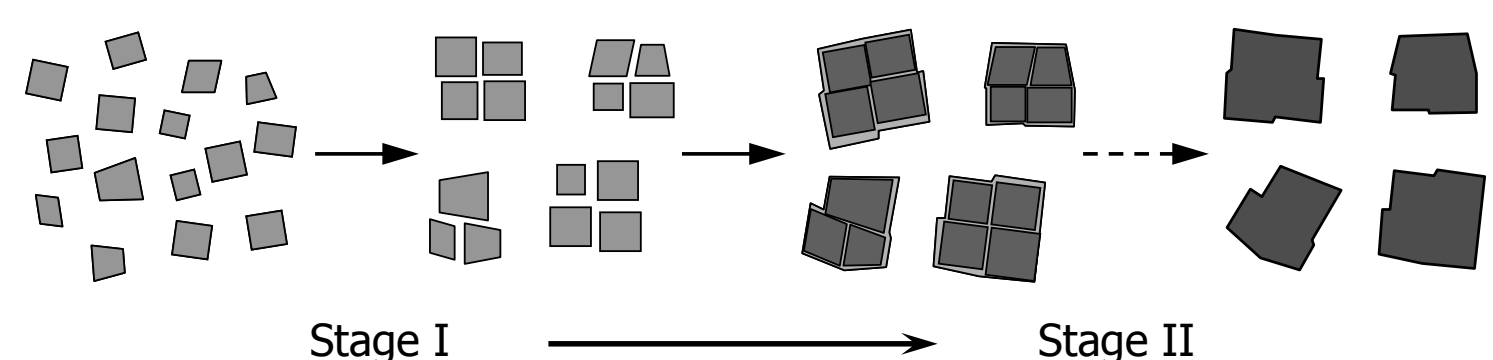

Figure 6-18. Schematic illustration of the growth mechanism for nanoparticles in the low-concentration reaction.

Platinum monomers first nucleate and grow into faceted primary crystallites, which then grow through oriented attachment, forming attached nanocrystals. The attached nanocrystals continue to grow with free platinum atoms filling up the gaps between the primary crystallites.

As mentioned, the growth rate of $\left[\mathrm{Pt}_{\mathrm{NC}}\right]$ is much slower for the low-concentration reaction, compared to that for the high-concentration reaction. TEM observations of nanoparticles from the different reaction times suggest that the faceted and non-branched morphologies are maintained throughout the reaction. Slow nanocrystal growth and formation of non-branched morphologies have been shown to be characteristic of growth in a thermodynamically controlled growth regime.[18-20] With the low precursor concentration, a low solution concentration of free platinum atoms is produced, resulting in relatively slow nanocrystal growth. The growth is also shown to involve oriented attachments of faceted crystallites, leading to faceted nanocrystals of single crystalline structures. This irreversible process is understood to be thermodynamically favoured as high-energy surfaces (of much smaller nanocrystals) are eliminated resulting in the reduction of the surface free energy of the system. ${ }^{[21,22]}$ Nanocrystal growth in the low-concentration reaction therefore occurs 
under thermodynamic control involving an oriented attachment growth process, yielding stable morphologies.

\subsubsection{Growth Mechanism: High-Concentration Reaction}

The growth of platinum nanoparticles in the high-concentration reaction was observed to occur in Stages I-IV. Hence the growth mechanism is broken down into the four stages, and is discussed based on results obtained from both in situ and off-line experiments.

\section{Stage I. Formation of quasi-octapods}

The XRD data showed that the increase of $\left[\mathrm{Pt}_{\mathrm{NC}}\right]$ during Stage I was rapid and there was fast growth of nanocrystals. A fast growth rate has been associated with kinetically controlled growth, which often leads to a complex growth pattern and nanocrystals having branched morphologies. ${ }^{[19]}$ Interpretation from TEM imaging suggests that the platinum nanocrystals nucleate and grow into quasi octapods during this stage. For fcc metals such as gold and platinum, a single-crystalline nanocrystal often originates from a cuboctahedral nucleus or seed that is enclosed by six $\{100\}$ facets and eight $\{111\}$ facets.[23,24] The observed quasioctapods are believed to be a result of faster growth on the $\{111\}$ facets than on the $\{100\}$ facets, giving rise to the short arms along the $\langle 111\rangle$ directions.

\section{Stage II. Quasi-octapods $\rightarrow$ etched-octapods}

There was almost no change in the total amount of platinum atoms in the nanocrystals ([Pt $\left.\left.\mathrm{PC}_{\mathrm{NC}}\right]\right)$ in Stage II. TEM images showed that the arms of the quasi-octapods had grown further along the $\langle 111\rangle$ directions, while the cross-section towards the centre of the $\{100\}$ facets had reduced. The growth on the quasi-octapods thus took place over the arms along the $\langle 111\rangle$ directions, while etching had occurred on the $\{100\}$ facets, leading to the formation of etched-octapods.

By correlating the observations in XRD and TEM, it can be seen that the increase in [ $\left.\mathrm{Pt}_{\mathrm{NC}}\right]$ due to nanocrystal growth is balanced by the loss of re-dissolved platinum atoms from the nanocrystals as a consequence of etching; hence the peak area remains constant over this growth stage. Similarly, the near-constant X-ray correlation length as observed in 
Figure 6-7 is evidence of an increase and decrease in the crystallite size that effectively average out to no change.

These observations thus suggest that both growth and etching processes were occurring selectively, simultaneously, and at comparable rates.

\section{Stage III. Etched-octapods $\rightarrow$ porous nanocrystals}

The relatively rapid increase in $\left[\mathrm{Pt}_{\mathrm{NC}}\right]$ in Stage III was similar to that observed in Stage I, which indicated that the growth at this stage again occurred under kinetic control. However, the slight decrease in $L$ suggested an unconventional type of growth. As observed in the TEM images (Expt Pt-4C), a significant change in the particle morphology gradually took place, which was characterized by etching of much of the central core and the formation of more branches. These changes led to an increase of porosity in the nanocrystals. As the nanocrystals became increasingly porous, the average size of the single crystalline domains, and hence $L$, decreased.

\section{Stage IV. Growth of porous nanocrystals}

Both XRD and TEM results suggested that the porous nanocrystals continued to grow but at a relatively slower rate compared to Stages I and III. The relatively slow growth could be understood as a result of monomer depletion in the solution. TEM imaging showed that there was increase in both overall nanoparticle size and branches on the porous nanocrystals at 500 minutes. This means increase in the $\left[\mathrm{Pt}_{\mathrm{Nc}}\right]$ and degree of porosity, resulting in a slight decrease in $L$.

It is believed that multiple branching of the porous nanocrystals also induces twin defects into the nanocrystals, resulting in the formation of polycrystalline structures. This is believed to be occurring towards the later part of Stage IV, in which multiply-branched nanoparticles began to form. The polycrystalline nature of the multiply-branched nanoparticles also implied the occurrence of aggregation. 
Therefore, a growth mechanism of branched platinum nanoparticles that is under kinetic control has been observed, which involves rapid growth and selective etching. The scheme in Figure 6-19 illustrates the proposed growth mechanism from Stage I to III.

It is interesting to note that at Stage I, the TEM average nanocrystal size $(6.5 \mathrm{~nm})$ agrees well with the XRD coherence length $L(6 \mathrm{~nm})$, confirming the single crystal nature of the quasi-octapod nanoparticles. In contrast, in Stages II-IV, $L$ is noticeably smaller than the TEM average nanocrystal size. Thus the variance between the $L$ and the nanoparticle size obtained from the TEM images is a useful measure of nanoparticle porosity.

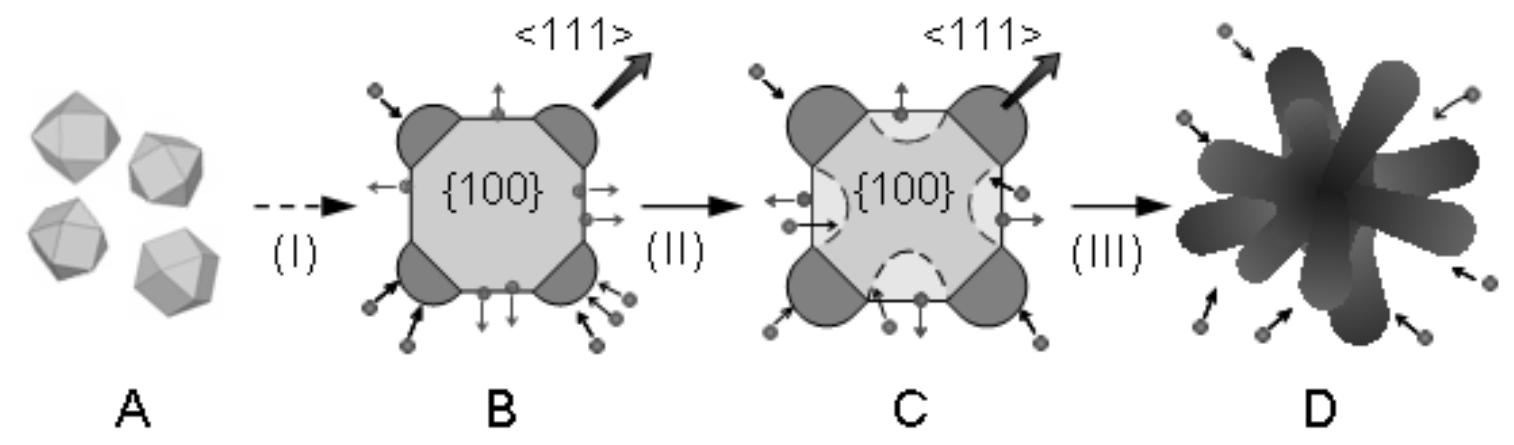

Figure 6-19. Schematic illustrates the proposed growth mechanism of platinum nanocrystals in the high-concentration reaction.

Growth is illustrated for the first three stages (I - III). Platinum monomers first nucleate into cuboctahedral nuclei (A), and then grow into single-crystalline quasi-octapods (B). Growth of the arms of the quasi-octapods, coupled with selective etching on the edges and centres of facets leads to formation of etched-octapods (C). These processes continue and transform the nanocrystals to porous nanocrystals (D).

\subsubsection{The Etching Process in High-Concentration Reaction}

During the growth of the platinum nanocrystals one point of particular interest is Stage II where simultaneous, selective growth and etching take place at comparable rates leading to shape transformation, but no overall nanocrystal growth.

In terms of a possible etchant species, a mixture of surfactant, solvent, and precursor are present in solution-phase synthesis. Since etching is only observed only in the reaction of high precursor concentration, it is likely that the etchant originates from the precursor, which can be the acetylacetonate or a decomposition/reaction by-product of acetylacetonate that could perform oxidative etching of platinum. Etching most likely commences during Stage II, rather abruptly beginning at $\sim 100$ minutes. At this point in the 
reaction, it is believed that enough of the platinum acetylacetonate has reacted to produce a sufficiently high concentration of the etchant to begin etching the nanocrystals.

At 180 minutes (end of Stage II) the etching was observed to have stopped and growth continued rapidly. There could be two reasons for the end of Stage II growth. First, it was observed that etching took place almost exclusively on the $\{100\}$ facets, and hence it was unlikely to occur when no $\{100\}$ facets were available. Thus, the etching process was retarded at the end of Stage II when most $\{100\}$ facets had disappeared. Second, when enough atoms on the $\{100\}$ facets were removed, high-index facets together with kinkedand stepped-faces were exposed. The newly exposed faces are believed to be the main driving factor for the rapid growth that commenced in Stage III. It is known that kinks and steps can induce preferential and spontaneous growth, resulting in much faster growth rates on faces with these defects.[25,26]

\subsection{Chapter Summary}

Growth and evolution of platinum nanoparticles in solutions of different precursor concentration were investigated by both in situ XRD and ex situ TEM analyses.

The low-concentration reaction has been shown to occur under a thermodynamically controlled regime. The growth mechanism involved an oriented attachment growth process, with faceted morphologies being maintained throughout the reaction.

In a reaction with high precursor concentration, growth was more complex and has been shown to be under kinetic control, resulting in multiply-branched nanoparticles. The results and analyses have demonstrated that growth in the high-concentration reaction involved a structural transformation mechanism for octapod-like nanocrystals to grow into porous nanostructures. Selective growth and etching occurring during early growth stages have been shown to be responsible for the transformation. 


\subsection{Experimental}

\section{Materials}

All reagents were used as received without further purification. Platinum(II) acetylacetonate $\left(\operatorname{Pt}(\mathrm{acac})_{2}\right)$ was purchased from Sigma-Aldrich, and oleylamine (technical, $\geq 70 \%$ ) was purchased from Fluka. Toluene and methanol were of reagent grade.

\section{In situ experiment}

In a typical experiment, the high-precursor concentration solution was prepared by dissolving $0.05 \mathrm{mmol}(0.0196 \mathrm{~g})$ of $\mathrm{Pt}(\mathrm{acac})_{2}$ in $1 \mathrm{~mL}$ of toluene, giving a $0.05 \mathrm{M}$ solution, to which $0.5 \mathrm{mmol}(0.165 \mathrm{~mL})$ of oleylamine was added. For the low-concentration reaction, $0.1 \mathrm{~mL}$ of the $0.05 \mathrm{M}$ solution was diluted by adding toluene to make up a $1 \mathrm{~mL}$ solution, giving $0.005 \mathrm{M}$. The prepared precursor solution was injected into the XRD cell and in situ experiments were conducted as described in Chapter 2, Sections 2.1.2 and 2.2.1.

\section{Ex situ synthesis}

In a typical high-concentration reaction, $0.1 \mathrm{mmol}(0.039 \mathrm{~g})$ of $\mathrm{Pt}(\mathrm{acac}) 2$ was weighed in a vial, then added with $2 \mathrm{~mL}$ of toluene and $1 \mathrm{mmol}(0.33 \mathrm{~mL})$ of oleylamine. The vial was placed in a Fischer-Porter bottle. For the low-concentration reaction, $0.05 \mathrm{mmol}$ (0.0196 g) of Pt(acac)2 was weighed and transferred to a Fischer-Porter bottle, then added with $10 \mathrm{~mL}$ of toluene and $0.5 \mathrm{mmol}(0.165 \mathrm{~mL})$ of oleylamine. The Fischer-Porter bottle was flushed 3 times with hydrogen gas $\left(\mathrm{H}_{2}\right)$ before being filled with $200 \mathrm{kPa} \mathrm{H}_{2}$, and sealed. The bottle was placed into an oven heated at $70^{\circ} \mathrm{C}$ for the desired reaction time.

For ex situ experiments carried out in the XRD cell, preparation was the same as that for the in situ experiments. Once prepared the cell was placed into an oven heated at $70{ }^{\circ} \mathrm{C}$.

\section{Purification}

The reaction was allowed to cool to room temperature naturally before the Fischer-Porter bottle or the XRD cell was opened to air. Equivalent volume methanol was added to the reaction mixture to flocculate and precipitate the nanoparticles. The mixture was centrifuged at $10 \mathrm{krpm}$ for 5 minutes to isolate a black solid. The solid was re-dispersed in 
toluene and the precipitation and centrifugation steps repeated. The final product was collected and stored as a powder.

\section{TEM characterization}

The samples for TEM studies were prepared by resuspending the precipitate in toluene. One drop of the toluene suspension was put onto a carbon-supported copper grid and allowed to evaporate under ambient conditions.

\subsection{References}

[1] J. Ren and R. D. Tilley, Preparation, self-assembly, and mechanistic study of highly monodispersed nanocubes. J. Am. Chem. Soc. 129, 3287-3291 (2007).

[2] J. Ren and R. D. Tilley, Shape-controlled growth of platinum nanoparticles. Small 3, 1508-1512 (2007).

[3] T. S. Ahmandi, Z. L. Wang, T. C. Green, A. Henglein and M. A. El-Sayed, Shapecontrolled synthesis of colloidal platinum nanoparticles. Science 272, 1924-1926 (1996).

[4] A. Miyazaki and Y. Nakano, Morphology of platinum nanoparticles protected by poly( $n$-isopropylacrylamide). Langmuir 16, 7109-7111 (2000).

[5] H. Song, F. Kim, S. Connor, G. A. Somorjai and P. Yang, Pt nanocrystals: shape control and Langmuir-Blodgett monolayer formation. J. Phys. Chem. B 109, 188-193 (2005).

[6] N. Tian, Z.-Y. Zhou, S.-G. Sun, Y. Ding and Z. L. Wang, Synthesis of tetrahexahedral platinum nanocrystals with high-index facets and high electro-oxidation activity. Science 316, 732-735 (2007).

[7] D. Fenske, H. Borchert, J. Kehres, R. Kroger, J. Parisi and J. Kolny-Olesiak, Colloidal synthesis of Pt nanoparticles: on the formation and stability of nanowires. Langmuir 24, 9011-9016 (2008).

[8] C. Burda, X. Chen, R. Narayanan and M. A. El-Sayed, Chemistry and properties of nanocrystals of different shapes. Chem. Rev. 105, 1025-1102 (2005). 
[9] J. Park, J. Joo, S. G. Kwon, Y. Jang and T. Hyeon, Synthesis of monodisperse spherical nanocrystals. Angew. Chem. Int. Ed. 46, 4630-4660 (2007).

[10] M.-P. Pileni, Control of the size and shape of inorganic nanocrystals at various scales from nano to macrodomains. J. Phys. Chem. C 111, 9019-9038 (2007).

[11] J. M. Petroski, Z. L. Wang, T. C. Green and M. A. El-Sayed, Kinetically controlled growth and shape formation mechanism of platinum nanoparticles. J. Phys. Chem. B 102, 3316-3320 (1998).

[12] T. Herricks, J. Chen and Y. Xia, Polyol synthesis of platinum nanoparticles: control of morphology with sodium nitrate. Nano Lett. 4, 2367-2371 (2004).

[13] J. Rodriguez-Fernandez, J. Perez-Juste, P. Mulvaney and L. M. Liz-Marzan, Spatiallydirected oxidation of gold nanoparticles by Au(III)-CTAB complexes. J. Phys. Chem. $B$ 109, 14257-14261 (2005).

[14] L. Qu, W. W. Yu and X. Peng, In situ observation of the nucleation and growth of CdSe nanocrystals. Nano Lett. 4, 465-469 (2004).

[15] R. Viswanatha, H. Amenitsch and D. D. Sarma, Growth kinetics of ZnO nanocrystals: a few surprises. J. Am. Chem. Soc. 129, 4470-4475 (2007).

[16] K. Biswas, N. Varghese and C. N. R. Rao, Growth kinetics of gold nanocrystals: a combined small-angle X-ray scattering and calorimetric study. Small 4, 649-655 (2008).

[17] X. Teng, X. Liang, S. Maksimuk and H. Yang, Synthesis of porous platinum nanoparticles. Small 2, 249-253 (2006).

[18] Y. Xia, Y. Xiong, B. Lim and S. E. Skrabalak, Shape-controlled synthesis of metal nanocrystals: simple chemistry meets complex physics? Angew. Chem. Int. Ed. 48, 60103 (2009).

[19] Y. Yin and A. P. Alivisatos, Colloidal nanocrystal synthesis and the organic-inorganic interface. Nature 437, 664-670 (2005).

[20] G. Berhault, M. Bausach, L. Bisson, L. Becerra, C. Thomazeau and D. Uzio, Seedmediated synthesis of $\mathrm{Pd}$ nanocrystals: factors influencing a kinetic- or thermodynamic-controlled growth regime. J. Phys. Chem. C 111, 5915-5925 (2007). 
[21] R. L. Penn, G. Oskam, T. J. Strathmann, P. C. Searson, A. T. Stone and D. R. Veblen, Epitaxial assembly in aged colloids. J. Phys. Chem. B 105, 2177-2182 (2001).

[22] J. F. Banfield, S. A. Welch, H. Zhang, T. T. Ebert and R. L. Penn, Aggregation-based crystal growth and microstructure development in natural iron oxyhydroxide biomineralization products. Science 289, 751-754 (2000).

[23] Z. L. Wang, Transmission electron microscopy of shape-controlled nanocrystals and their assemblies. J. Phys. Chem. B 104, 1153-1175 (2000).

[24] S. Maksimuk, X. Teng and H. Yang, Roles of twin defects in the formation of platinum multipod nanocrystals. J. Phys. Chem. C 111, 14312-14319 (2007).

[25] A. A. Chernov, Crystal growth and crystallography. Acta Crystallogr. A54, 859-872 (1998).

[26] C. R. Henry, Morphology of supported nanoparticles. Prog. Surf. Sci. 80, 92-116 (2005). 


\section{Chapter 7}

\section{Conclusions and Future Work}

\section{Chapter outline}

\subsection{Conclusions}

7.1.1. Fischer-Porter Bottle Synthesis

7.1.2. Growth of Nanoparticles in Solution

7.2. Future Work

7.3. References

\subsection{Conclusions}

This thesis is concerned with the synthesis and characterisation of nanoparticles, and the growth of nanoparticles in solution. Materials involved were iron, iron carbide, ruthenium and platinum.

The synthesis of iron, iron carbide and ruthenium nanoparticles using Fischer-Porter bottles was explored and investigated by employing different experimental conditions. Although these materials crystallise in different structures, the effects of different reaction conditions on the synthesis results were observed to be similar or closely related, and are discussed in Section 7.1.1 below. Observations specific to the synthesis of iron and iron carbide nanoparticles, and the synthesis of ruthenium nanoparticles, are summarised in the respective sub-sections.

A detailed study was undertaken to investigate the growth of platinum nanoparticles in solution, employing in situ synchrotron-based XRD and ex situ TEM techniques. This part of the research work is summarised in Section 7.1.2. 


\subsubsection{Fischer-Porter Bottle Synthesis}

In this study, a facile synthesis route was developed in producing nanoparticles of iron, iron carbide and ruthenium. The nanoparticles synthesised were highly crystalline, and in most cases were observed to be of single-crystal structures. Factors that contributed to the formation of nanoparticles of different sizes and shapes include precursor concentration, the type and amount of stabilisers used, the reaction temperature and time.

Syntheses were carried out in Fischer-Porter bottles. The use of Fischer-Porter bottles for nanoparticle synthesis (including those of iron and ruthenium) has been mainly reported by Chaudret and co-workers since late 1990's.[1,2] However, the reported synthesis method requires that the Fischer-Porter bottle setup be prepared in a glove box. This is mainly because the precursors involved are often volatile and/or air-sensitive. In the present study, the precursors used were relatively stable in air hence preparation of the Fischer-Porter bottles was carried out in fume cupboards. Not only that, the use of different precursors in this study has also produced nanoparticles of various morphologies including some unconventional ones.

\section{General trend}

The control of nanoparticle size was observed to be related to the amount of stabiliser used. The increase of the amount of oleylamine as a stabilising agent resulted in the increase of the size of iron and iron carbide nanoparticles. However, the opposite effect was observed for the synthesis of ruthenium nanoparticles, in which smaller nanoparticles were produced when larger amount of alkylamine was used.

For the formation of iron and iron carbide nanoparticles, the involvement of the stabilising molecules in the reaction pathway of the precursor was implied by the results obtained. In larger presence of the stabiliser, the interaction between the stabilising molecules and the precursor and/or the partially decomposed/reacted precursor was believed to have inhibited the nucleation process and reduced the number of nuclei formed. Larger nanoparticles were thus produced due to greater supply of monomer over time as the precursor continued to decompose/react slowly.

Involvement of the amine stabiliser in the reaction pathway of the precursor was not indicated for the synthesis of ruthenium nanoparticles. The role of the alkylamine was 
observed to be merely a stabilising agent. Stronger stabilisation effect was demonstrated by the smaller nanoparticles obtained from reactions with larger presence of the stabiliser. The effect of temperature on the nanoparticle size was observed to be related to the role played by the stabiliser in the synthesis. The size of iron and iron carbide nanoparticles was observed to decrease with the increase of temperature. In contrast, larger ruthenium nanoparticles were produced from reactions with higher temperatures.

\section{Type of reactions involved}

Syntheses were mainly conducted under a reducing (hydrogen) atmosphere. Some experiments were repeated under an inert (nitrogen) atmosphere, which provided an indication of the type of reactions involved in the synthesis.

For nanoparticle synthesis that required the presence of hydrogen, such as that of ruthenium nanoparticles from the reaction of $\mathrm{Ru}(\mathrm{MA})_{2}(\mathrm{COD})^{*}$, the reaction of the precursor was deduced to be of reduction. The reaction of the iron precursor $\mathrm{Fe}\left(\mathrm{C}_{5} \mathrm{H}_{5}\right)\left(\mathrm{C}_{6} \mathrm{H}_{7}\right)$, in contrast, was deduced to be of thermal decomposition, as the nanoparticles were observed to be the same when only reaction atmosphere was varied. Through the comparison of the synthesis results under the different atmospheres, a trend of the nanoparticle size distribution was observed, as summarised in Table 7-1. Syntheses involved with the reduction of precursors in general were observed to produce nanoparticles with small size distribution. A monodisperse sample was more difficult to achieve with syntheses involving mainly thermal decomposition of the precursors.

It has been reported that in Fischer-Porter bottle synthesis, nucleation and growth of nanoparticles under a hydrogen atmosphere are much slower than those involved with other solution methods, such as thermal decomposition of a precursor in a three-necked flask. ${ }^{[3,4]}$ It is thus believed that in this study, when nanoparticle synthesis involved mainly thermal decomposition of the precursor, the nucleation and growth of nanoparticles occurred at higher rates and hence in a less controlled fashion.

Perhaps several different reactions were involved in each experiment. Nevertheless, the type of reactions as implied by the results was merely concerned with the synthesis of nanoparticles investigated. It is hoped that the above observations could aid in the design

* MA = 2-methylallyl; COD = 1,5-cyclooctadiene 
and planning of future synthetic strategies involving Fischer-Porter bottle synthesis and the like.

Table 7-1. Comparison of size distribution achieved through different reactions.

\begin{tabular}{|l|l|l|}
\hline Precursor & $\begin{array}{l}\text { Type of reaction involved in } \\
\text { nanoparticle synthesis }\end{array}$ & $\begin{array}{l}\text { Size distribution of NPs } \\
\text { from an average synthesis }\end{array}$ \\
\hline $\mathrm{Fe}\left(\mathrm{C}_{5} \mathrm{H}_{5}\right)\left(\mathrm{C}_{6} \mathrm{H}_{7}\right)$ & Thermal decomposition & Large \\
\hline $\mathrm{FeCl}_{3}$ & Reduction & Small \\
\hline $\mathrm{Ru}(\mathrm{MA})_{2}(\mathrm{COD})$ & Reduction & Small \\
\hline $\mathrm{Ru}(\mathrm{acac})_{3}$ & Reduction and thermal decomposition & Medium \\
\hline $\mathrm{Pt}(\mathrm{acac})_{2}$ & Reduction & Small \\
\hline
\end{tabular}

₹ Small, medium and large correspond to different range of standard deviation, $\sigma$; Small: $\sigma \leq 15 \%$; Medium: $15 \%<\sigma<25 \%$; Large: $\sigma \geq 25 \%$.

\section{Iron and iron carbide nanoparticles}

Highly crystalline nanoparticles of $\alpha$-Fe and $\mathrm{Fe}_{3} \mathrm{C}$ were successfully synthesised, as described in Chapters 3 and 4. Depending on the reaction conditions, near-monodisperse core/shell nanoparticles in the range of 14-25 nm were obtained. An oxide layer of 3-4 nm was found to have formed instantly on the nanoparticle surface upon exposure to air. Further oxidation was observed to be depending on time and size of nanoparticles. One of the advantages shown in this study over other syntheses reported in the literature is that nanoparticles obtained are generally larger in size. The relatively larger nanoparticle size thus means greater stability of the nanoparticles. This allows post-synthesis processes such as purification and surface modification be carried out under ambient conditions.

Perhaps one of the most significant observations during this research is the formation of $\mathrm{Fe}_{3} \mathrm{C}$ at temperatures as low as $110^{\circ} \mathrm{C}$. The reaction conditions involved are much milder compared to other methods in the literature, in which $350{ }^{\circ} \mathrm{C}$ has been the lowest reported.[5]

\section{Ruthenium nanoparticles}

Single-crystal hcp ruthenium nanoparticles were successfully synthesised through the reactions of precursors $\mathrm{Ru}(\mathrm{MA})_{2}(\mathrm{COD})$ and $\mathrm{Ru}(\mathrm{acac})_{3}$ in Fischer-Porter bottles, as described 
in Chapter 5. Nanoparticle morphologies observed include spherical and near-spherical, rod- and worm-like, tripod and plate-like structures. Morphological control was achieved by varying the stabilisation conditions. Non-branched morphologies such as spherical and near-spherical structures were mainly observed for nanoparticles stabilised by alkylamine, whereas tripod nanoparticles were formed in the presence of oleic acid. When a small amount of stabiliser was used, formation of one-dimensional morphologies was promoted. Rod-like nanoparticles were shown to be a result of growth along the $\langle 001\rangle$ directions (the $c$-axis) of the hcp structure.

\subsubsection{Growth of Nanoparticles in Solution}

Growth and evolution of platinum nanoparticles of different morphologies were investigated using both in situ and ex situ techniques, as presented in Chapter 6. The study demonstrated the utility of TEM and in situ XRD for revealing the growth details of different morphologies.

Growth of faceted nanocrystals in the low-concentration reaction was shown to occur under a thermodynamically controlled regime. Nanoparticle growth involved an oriented attachment mechanism, in which cube-like crystallites of $\sim 6 \mathrm{~nm}$ assembled and attached in iso-orientation to form nanocrystals of similar morphologies but of much larger size of $\sim 12 \mathrm{~nm}$. The growth of branched and porous nanoparticles in the high-concentration reaction was shown to be more complex, under a kinetically controlled regime. It was demonstrated that nanoparticle growth involved a structural transformation of octapod-like nanocrystals into porous nanostructures. A new mechanism involving selective growth and etching that occurred during early growth stages has been shown to be responsible for the transformation.

\subsection{Future Work}

This study has shown that nanoparticles of iron, iron carbide and ruthenium were synthesised via a facile chemical route using Fischer-Porter bottles.

The synthetic methodology developed could be employed for the synthesis of nanoparticles of other metals such as cobalt and nickel, and the synthesis of nanoparticles of metal 
carbide such as nickel carbide and molybdenum carbide. Organometallic compounds similar to the organoiron complex used in this study could be explored for the synthesis of carbide nanoparticles.

Suggested future work for the investigation of iron and iron carbide nanoparticles essentially centres around the mechanistic studies and further characterisations of the various core/shell nanoparticles. In particular, nanoparticles of interesting morphologies such as the dimer- and dumbbell-like structures. Perhaps the reactions could be quenched at different stages and the intermediate crystallites be characterised. Of course, the post-synthesis work would have to be done with extreme care in order to prevent oxidation process. Another approach might be an in situ study, which could adopt the techniques developed in the growth study of platinum nanoparticles. Further characterisation work will aim at gaining information about the composition of the sample as a whole, particularly products consisting of a mixture of $\alpha$-Fe and $\mathrm{Fe}_{3} \mathrm{C}$ nanoparticles. For example, Mossbauer spectroscopy could be used in conjunction with the XRD and SAED data obtained in this study to provide further information of the relative phases formed under the different reaction conditions.

The results from magnetic measurement of selected samples of iron-based nanoparticles showed that these nanoparticles have the potential to be modified on the surface to exhibit ferro- or superparamagnetic behaviour. Hence the next step would be to examine the effect of surface modification on the magnetic properties. Different capping ligands have been used for coating iron oxide nanoparticles, thus the same approach could be applied on the core/shell nanoparticles in this study. This will also provide an assessment of the availability of the nanoparticles for various magnetic applications.

The synthesis of ruthenium nanoparticles can be further investigated. The synthesis through the reaction of $\mathrm{Ru}(\mathrm{acac})_{3}$ might be tuned to produce nanorods and branched structures of different sizes and aspect ratios.

One of the common conditions shared by the syntheses of both iron and ruthenium nanoparticles is the reaction temperature. This implies that both precursors could react at the same temperature to produce bimetallic nanoparticles of $\mathrm{Fe}-\mathrm{Ru}$, which have been shown to possess unique catalytic properties.[6,7] Thus one possible future work would be to 
investigate the synthesis of Fe-Ru bimetallic nanoparticles, of dimer or core/shell structures.

The methodology developed in studying the growth of nanocrystals in solution could be extended to nanoparticles of other crystal structures. One possible candidate is ruthenium nanoparticles, of which different morphologies were observed in this study. Study could be conducted to investigate the growth of the tripod and zigzag rod-like structures. The outcome is believed to be applicable to other metals of hcp structures that are known to form one-dimensional structures, such as cobalt. The information gained from different systems could eventually lead to a complete and general mechanistic understanding of nanoparticle growth in solution.

\subsection{References}

[1] M. Respaud, J. M. Broto, H. Rakoto, A. R. Fert, L. Thomas, B. Barbara, M. Verelst, E. Snoeck, P. Lecante, A. Mosset, J. Osuna, T. O. Ely, C. Amiens and B. Chaudret, Surface effects on the magnetic properties of ultrafine cobalt particles. Phys. Rev. B 57, 29252935 (1998).

[2] O. Vidoni, K. Philippot, C. Amiens, B. Chaudret, O. Balmes, J.-O. Malm, J.-O. Bovin, F. Senocq and M.-J. Casanove, Novel, spongelike ruthenium particles of controllable size stabilized only by organic solvents. Angew. Chem. Int. Ed. 38, 3736-3738 (1999).

[3] F. Dumestre, B. Chaudret, C. Amiens, M.-C. Fromen, M.-J. Casanove, P. Renaud and P. Zurcher, Shape control of thermodynamic stable cobalt nanorods through organometallic chemistry. Angew. Chem. Int. Ed. 41, 4286-4289 (2002).

[4] F. Dumestre, B. Chaudret, C. Amiens, M. Respaud, P. Fejes, P. Renaud and P. Zurcher, Unprecedented crystalline super-lattices of monodisperse cobalt nanorods. Angew. Chem. Int. Ed. 42, 5213-5216 (2003).

[5] S. Yu and G. M. Chow, Synthesis, structural, magnetic, and cytotoxic properties of iron oxide coated iron/iron-carbide nanocomposite particles. J. Appl. Phys. 98, 114306 (2005). 
[6] M. Kaminsky, K. J. Yoon, G. L. Geoffroy and M. A. Vannice, Carbon-supported Fe-Ru catalysts prepared from stoichiometric mixed-metal carbonyl clusters. J. Catal. 91, 338351 (1985).

[7] M. C. Bahome, L. L. Jewell, K. Padayachy, D. Hildebrandt, D. Glasser, A. Datye and N. J. Coville, Fe-Ru small particles bimetallic catalysts supported on carbon nanotubes for use in Fischer-Tropsch synthesis. Appl. Catal., A 328, 243-251 (2007). 


\section{List of Publications}

[1] R. D. Tilley, S. Cheong and J. Ren, Magnetic Nanoparticles (Intellectual Property Office of New Zealand, NZ573797, 2008).

[2] S. Cheong, J. Watt, B. Ingham, M. F. Toney and R. D. Tilley, In situ and ex situ studies of platinum nanocrystals: growth and evolution in solution. J. Am. Chem. Soc. 131, 14590-14595 (2009).

[3] J. Watt, S. Cheong, M. F. Toney, B. Ingham, J. Cookson, P. T. Bishop and R. D. Tilley, Ultrafast growth of highly branched palladium nanostructures for catalysis. ACS Nano 4, 396-402 (2009). 